Supporting Information for:

\title{
Ruthenium-Catalyzed Asymmetric Allylic Alkylation of Isatins
}

Barry M. Trost, ${ }^{* \dagger}$ Christopher A. Kalnmals, ${ }^{\dagger}$ Divya Ramakrishnan, ${ }^{\dagger}$ Michael C. Ryan, ${ }^{\dagger}$ Rebecca W. Smaha, ${ }^{\dagger}$ and Sean Parkin

${ }^{\dagger}$ Department of Chemistry, Stanford University, Stanford, CA 94305, United States

$\star$ Department of Chemistry, University of Kentucky, Lexington, KY 40506, United States 


\section{Table of Contents}

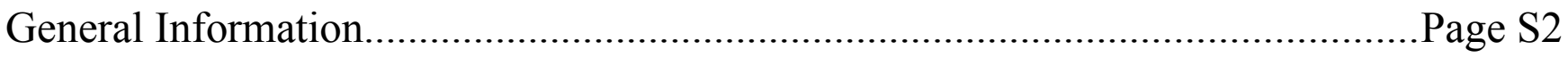

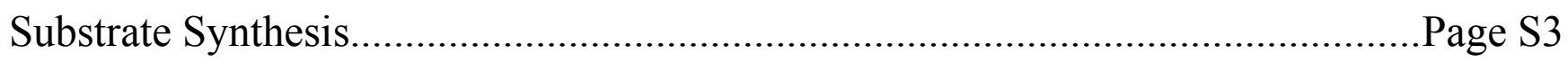

Ru-Catalyzed Asymmetric Allylic Alkylation Reactions....................................Page S9

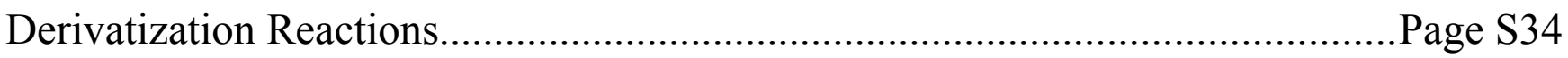

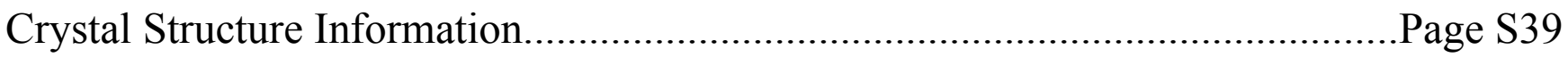

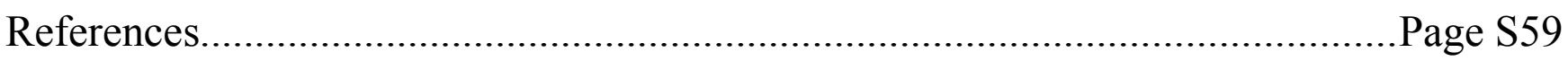

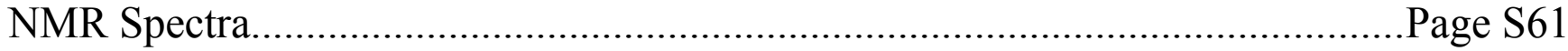




\section{General Information}

Unless otherwise noted, all reagents were purchased commercially and used as received. Anhydrous tetrahydrofuran (THF) was obtained by distillation from sodium/benzophenone ("freshly distilled") or from an AcroSeal bottle ("anhydrous"). Anhydrous dichloromethane $\left(\mathrm{CH}_{2} \mathrm{Cl}_{2}\right)$ was purchased as such from Acros Organics in AcroSeal bottles and was used as received. Diisopropylethylamine (DIPEA) and cinnamyl chloride were distilled in small batches and were stored under argon, but dispensed in ambient air. When performing air-sensitive reactions, reagents and solvents were transferred using either stainless steel cannulae or plastic syringes equipped with stainless steel needles. Air-sensitive reactions were performed under a positive pressure of either nitrogen $\left(\mathrm{N}_{2}\right)$ or argon $(\mathrm{Ar})$ in reaction vessels sealed with rubber septa.

Analytical thin-layer chromatography (TLC) was performed on glass-backed silica-coated plates (Merck TLC Silicagel 60 $\left.\mathrm{F}_{254}, 250 \mu \mathrm{m}\right)$. All reported $R_{\mathrm{f}}$ values were measured using freshly prepared eluent mixtures. Visualization was typically performed using UV light and/or basic potassium permanganate $\left(\mathrm{KMnO}_{4}\right)$. Purification by flash chromatography was performed on silica gel (Fisher Scientific, 230 - 400 mesh, grade 60) using bulk solvents. Purification by preparatory TLC was performed on glass-backed silica-coated plates (ChemScene PLC Silica Gel 60 GF254, $1000 \mu \mathrm{m}$ ), typically using HPLC grade solvents. Melting points were measured in open capillary tubes using a Thomas-Hoover Unimelt apparatus and are uncorrected.

Proton nuclear magnetic resonance ( ${ }^{1} \mathrm{H}$ NMR) spectra were recorded at $300 \mathrm{MHz}$ using a Varian Inova 300 spectrometer, at $400 \mathrm{MHz}$ using either a Varian 400 or Varian Mercury 400 spectrometer, or at $500 \mathrm{MHz}$ using a Varian Inova 500 spectrometer. All ${ }^{1} \mathrm{H}$ chemical shifts are reported in ppm relative to tetramethylsilane $(0.00 \mathrm{ppm})$ or the residual solvent peak (7.264 ppm for $\mathrm{CDCl}_{3}, 2.49 \mathrm{ppm}$ for DMSO- $d_{6}$ ). Multiplets were assigned with the assistance of the multiplet tool in Mestrenova, and are abbreviated as follows: $\mathrm{s}=$ singlet, $\mathrm{d}=$ doublet, $\mathrm{t}=$ triplet, $\mathrm{q}=$ quartet, $\mathrm{m}=$ multiplet, $\mathrm{b}=$ broad, app. $=$ apparent. Fluorine nuclear magnetic resonance $\left({ }^{19} \mathrm{~F}\right.$ NMR) spectra were recorded at $376 \mathrm{MHz}$ using either a Varian 400 or a Varian Mercury 400 spectrometer. Carbon nuclear magnetic resonance $\left({ }^{13} \mathrm{C} \mathrm{NMR}\right)$ spectra were recorded at $75 \mathrm{MHz}$ using a Varian Inova 300 spectrometer, at $101 \mathrm{MHz}$ using either a Varian 400 or a Varian Mercury 400 spectrometer, or at 126 $\mathrm{MHz}$ using a Varian Inova 500 spectrometer. All ${ }^{13} \mathrm{C}$ chemical shifts are reported in ppm relative to the center of the residual solvent peak (77.16 ppm for $\mathrm{CDCl}_{3}, 39.49 \mathrm{ppm}$ for DMSO- $d_{6}$ ). Where reported, ${ }^{13} \mathrm{C}$ peak assignments were made with the assistance of distortionless enhancement by polarization transfer (DEPT-135 and DEPT-90) spectra.

Infrared (IR) spectra were recorded on $\mathrm{NaCl}$ plates using a Nicolet IR100 FT-IR spectrometer and were typically measured as thin films from $\mathrm{CDCl}_{3}$. High resolution mass spectrometry (HRMS) was performed at University of Illinois - UrbanaChampaign using electrospray ionization (ESI) unless otherwise noted. Enantiomeric ratios (er) were determined by highperformance liquid chromatography (HPLC) on either an Agilent 1200 Series HPLC system or a Thermo SP Spectra SYSTEM P1000/SpectraSERIES UV100 HPLC system using the specified separation conditions. Unless otherwise noted, the detection wavelength was $254 \mathrm{~nm}$. Specific rotations $\left([\alpha]_{\mathrm{D}}\right)$ correspond to the reported er values, and were recorded at the specified temperature using the specified solvent and concentration on a Jasco P-1000 polarimeter equipped with a 50 mm cell. 


\section{Substrate Synthesis}

5,6-Dichloroisatin (7j)

The title compound was prepared according to the literature. ${ }^{1}$ A flask was charged with 6-chloroisatin $(1.0239 \mathrm{~g}, 5.639 \mathrm{mmol}, 1.00$ equiv) and a small crystal of iodine. The flask was sealed with a septum then evacuated and backfilled with $\mathrm{N}_{2}$ three times, then bulk $\mathrm{AcOH}(20 \mathrm{~mL})$ was added in a single portion and $\mathrm{SO}_{2} \mathrm{Cl}_{2}(0.96 \mathrm{~mL}, 11.8 \mathrm{mmol}, 2.1$ equiv) was added slowly via syringe. The resulting mixture was placed in a preheated 50 ${ }^{\circ} \mathrm{C}$ bath and stirred under an argon balloon overnight, during which time the reaction changed from yellow to orange. The following morning, $>95 \%$ conversion was observed by crude ${ }^{1} \mathrm{H}$ NMR. The reaction was cooled to room temperature and the precipitate was collected by vacuum filtration, rinsed with petroleum ether $(3 \times 10 \mathrm{~mL})$, and dried to afford the title compound as an orange powder that was pure by ${ }^{1} \mathrm{H}$ NMR and used without purification. The ${ }^{1} \mathrm{H}$ NMR data match the literature. ${ }^{1}(1.017 \mathrm{~g}, 83 \%)$

Physical appearance: Orange powder

Melting point: $>260^{\circ} \mathrm{C}$ (lit. $\left.273-275^{\circ} \mathrm{C}\right)^{2}$

${ }^{1}$ H NMR (400 MHz, DMSO-d $\left.\boldsymbol{d}_{6}\right): \delta 11.24(\mathrm{~s}, 1 \mathrm{H}), 7.78(\mathrm{~s}, 1 \mathrm{H}), 7.11$ (s, $\left.1 \mathrm{H}\right)$.

\section{(Benzyloxyimino)acetic acid (S1)}

$\mathrm{BnO}_{\mathrm{mN}}$ The title compound was prepared according to the literature. ${ }^{3}$ An Erlenmeyer flask was charged with $O$ H. ${ }^{\mathrm{OH}}$ benzylhydroxylamine hydrochloride $(12.569 \mathrm{~g}, 78.750 \mathrm{mmol}, 1.05 \mathrm{gequiv})$ and water $(750 \mathrm{~mL})$. After a

O homogeneous solution was obtained, glyoxylic acid monohydrate ( $6.904 \mathrm{~g}, 75.00 \mathrm{mmol}, 1.00$ equiv) was added in a single portion. After dissolution, the reaction became cloudy and a white precipitate formed in less than one minute. After stirring for 6 hours at room temperature under ambient atmosphere, the reaction was extracted with $\mathrm{CH}_{2} \mathrm{Cl}_{2}(150 \mathrm{~mL})$. The organic layer was dried over $\mathrm{MgSO}_{4}$ and concentrated in vacuo to a white powder that was pure by ${ }^{1} \mathrm{H}$ NMR and was used without purification. The ${ }^{1} \mathrm{H}$ NMR data match the literature. ${ }^{3}(10.107 \mathrm{~g}, 75 \%)$

Physical appearance: White crystalline solid

Melting point: $79-82{ }^{\circ} \mathrm{C}\left(\text { lit. } 78-80{ }^{\circ} \mathrm{C}\right)^{3}$

${ }^{1}$ H NMR (400 MHz, $\left.\mathbf{C D C l}_{3}\right): \delta 9.64(\mathrm{~s}, 1 \mathrm{H}), 7.54$ (s, 1H), 7.38 (m, 5H), 5.32 (s, 2H).

\section{2-(Benzyloxyimino)-1-(3,5-xylidino)-1-ethanone (S2)}

OBn A flame-dried flask was charged with (benzyloxyimino)acetic acid $\mathbf{S 1}$ (1.792 g, $10.00 \mathrm{mmol}, 1.00$ equiv), sealed with a septum, and evacuated and backfilled with $\mathrm{N}_{2}$ three times. Anhydrous $\mathrm{CH}_{2} \mathrm{Cl}_{2}(10 \mathrm{~mL})$ was added via syringe and the reaction was cooled to $0{ }^{\circ} \mathrm{C}$. Next, CDI (1.784 g, $11.00 \mathrm{mmol}, 1.10$ equiv) was quickly added in a single portion and the flask resealed, leading to vigorous gas evolution. After stirring for 10 minutes at 0 ${ }^{\circ} \mathrm{C}$, the ice bath was removed and the reaction was stirred for an additional 10 minutes before 3,5-dimethylaniline $(1.37 \mathrm{~mL}$, $11 \mathrm{mmol}, 1.1$ equiv) was added in a single portion via syringe. After 40 minutes, the reaction was diluted with $\mathrm{CH}_{2} \mathrm{Cl}_{2}$ and washed with $1 \mathrm{M} \mathrm{HCl}(20 \mathrm{~mL})$, leading to the formation of a small amount of precipitate that was not soluble in either layer. The organic layer was washed with saturated $\mathrm{NaHCO}_{3}(20 \mathrm{~mL})$, dried over $\mathrm{MgSO}_{4}$, and concentrated in vacuo to afford an orange oil. Purification by flash chromatography ( $80 \mathrm{~g}$ silica, 8:1 petroleum ether/EtOAc) accorded the title compound as a yellowish solid. The ${ }^{1} \mathrm{H}$ NMR data match the literature. ${ }^{4}(2.238 \mathrm{~g}, 79 \%)$

Physical appearance: Yellowish solid

TLC $R_{\mathbf{f}}(6: 1$ petroleum ether/EtOAc, $\mathbf{U V}): 0.41$

${ }^{1}$ H NMR (300 MHz, $\left.\mathbf{C D C l}_{3}\right): \delta 8.12(\mathrm{~s}, 1 \mathrm{H}), 7.54(\mathrm{~s}, 1 \mathrm{H}), 7.38(\mathrm{~m}, 5 \mathrm{H}), 7.21(\mathrm{~s}, 2 \mathrm{H}), 6.78(\mathrm{~s}, 1 \mathrm{H}), 5.25(\mathrm{~s}, 2 \mathrm{H}), 2.31(\mathrm{~s}, 6 \mathrm{H})$. 


\section{4,6-Dimethylisatin (7n)}

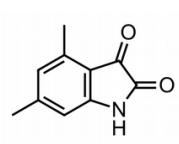

The title compound was prepared according to the literature. ${ }^{4}$ A flask was charged with concentrated $\mathrm{H}_{2} \mathrm{SO}_{4}$ $(8 \mathrm{~mL})$ and heated to $50{ }^{\circ} \mathrm{C}$ under ambient atmosphere. Over 30 minutes, oxime $\mathbf{S 2}$ was added portionwise with vigorous stirring, during which time the reaction changed from yellow to dark red. After the addition was complete, the reaction was stirred at $50{ }^{\circ} \mathrm{C}$ for 5 minutes, then the temperature was raised to $80{ }^{\circ} \mathrm{C}$. After stirring for 15 minutes at $80{ }^{\circ} \mathrm{C}$, the reaction was cooled to room temperature and poured into a mixture of crushed ice and water $(\sim 180$ $\mathrm{mL}$ ) with vigorous stirring. The initially formed red homogeneous solution slowly precipitated a very fine yellow powder over the next 15 minutes. This suspension was extracted with EtOAc $(4 \times 50 \mathrm{~mL})$, then the combined organic extracts were washed with saturated $\mathrm{NaHCO}_{3}(100 \mathrm{~mL})$, dried over $\mathrm{MgSO}_{4}$, and concentrated in vacuo to afford a yellow solid that was pure by ${ }^{1} \mathrm{H}$ NMR and used without purification. The ${ }^{1} \mathrm{H}$ NMR data match the literature. ${ }^{4}(320 \mathrm{mg}, 23 \%)$

Physical appearance: Yellow powder

Melting point: $236-242{ }^{\circ} \mathrm{C}$ (lit. $\left.241-243^{\circ} \mathrm{C}\right)^{5}$

${ }^{1}$ H NMR (400 MHz, DMSO-d $)$ ) $\delta 10.95$ (s, 1H), 6.68 (s, 1H), 6.52 (s, 1H), 2.38 (s, 3H), 2.28 (s, 3H).

\section{5-Azatricyclo[7.4.0.0 $\left.{ }^{2,6}\right]$ trideca-1(9),2(6),7,10,12-pentaene-3,4-dione (7o)}

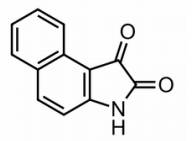

The title compound was prepared according to the literature. ${ }^{6} \mathrm{~A}$ flask was charged with 2-naphthylamine (500 mg, $3.49 \mathrm{mmol}, 1.00$ equiv) and bulk AcOH $(31 \mathrm{~mL})$, then diethyl ketomalonate $(0.59 \mathrm{~mL}, 3.8 \mathrm{mmol}$,

1.1 equiv) was added in a single portion. The resulting mixture was placed in a preheated $120{ }^{\circ} \mathrm{C}$ bath and stirred under ambient atmosphere for 4 hours, at which point the reaction was cooled to room temperature and concentrated in vacuo to afford a brown solid. To this solid was added a solution of $\mathrm{KOH}(2.410 \mathrm{~g}, 42.90 \mathrm{mmol}, 12.30$ equiv) in water $(46 \mathrm{~mL})$, and the resulting solution was stirred vigorously overnight under a gentle stream of air. The following day, some water was added to replace the evaporated solvent, then the reaction was acidified with $6 \mathrm{M} \mathrm{HCl}(\sim 8 \mathrm{~mL})$, leading to gas evolution and the formation of a dark red precipitate. This material was collected by vacuum filtration, rinsed with water, and dried to afford a dark red solid $(581 \mathrm{mg})$. Recrystallization of this crude material from refluxing $\mathrm{MeOH}(\sim 80 \mathrm{~mL})$ afforded pure title compound as red needles. The ${ }^{1} \mathrm{H}$ NMR data match the literature. ${ }^{6}$ (404 mg, 59\%)

Physical appearance: Red needles

Melting point: $246-248^{\circ} \mathrm{C}\left(\text { lit. } 250-251^{\circ} \mathrm{C}\right)^{7}$

${ }^{1}$ H NMR (300 MHz, DMSO-d $\left.\mathbf{d}_{6}\right): \delta 11.05(\mathrm{~s}, 1 \mathrm{H}), 8.39(\mathrm{~d}, J=8.3 \mathrm{~Hz}, 1 \mathrm{H}), 8.24(\mathrm{~d}, J=9.0 \mathrm{~Hz}, 1 \mathrm{H}), 7.92(\mathrm{~d}, J=8.2 \mathrm{~Hz}$, 1H), 7.66 (ddd, $J=8.3,7.0,1.3 \mathrm{~Hz}, 1 \mathrm{H}), 7.42(\mathrm{ddd}, J=8.2,6.9,1.2 \mathrm{~Hz}, 1 \mathrm{H}), 7.17$ (d, $J=8.6 \mathrm{~Hz}, 1 \mathrm{H})$.

\section{1-(o-Tolyl)-2-propen-1-ol (S3)}

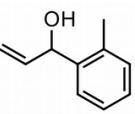

A flame-dried flask under $\mathrm{N}_{2}$ was charged with freshly distilled $o$-tolualdehyde $(1.16 \mathrm{~mL}, 10.0 \mathrm{mmol}, 1.00$ equiv) and freshly distilled THF $(10 \mathrm{~mL})$ and the resulting solution was cooled to $0{ }^{\circ} \mathrm{C}$. Vinylmagnesium bromide (1.0 M in THF; $10.5 \mathrm{~mL}, 10.5 \mathrm{mmol}, 1.05$ equiv) was added dropwise over a period of 10 minutes. The reaction was allowed to warm to room temperature as the ice bath expired, and after 1.5 hours was quenched with saturated $\mathrm{NH}_{4} \mathrm{Cl}(20 \mathrm{~mL})$. The layers were separated, then the aqueous phase was extracted with $\mathrm{Et}_{2} \mathrm{O}(3 \times 10 \mathrm{~mL})$. The combined organic layers were washed with saturated $\mathrm{NaCl}(30 \mathrm{~mL})$, dried over $\mathrm{MgSO}_{4}$, and concentrated in vacuo to afford a yellow oil. Purification by flash chromatography (30 $\mathrm{g}$ silica, 6:1 petroleum ether/EtOAc) afforded the title compound as a clear colorless oil. The ${ }^{1} \mathrm{H}$ NMR data match the literature. ${ }^{8}(1.291 \mathrm{~g}, 87 \%)$

Physical appearance: White solid

TLC $R_{\mathbf{f}}$ (5:1 petroleum ether/EtOAc, $\left.\mathbf{U V}\right): 0.34$

${ }^{1} \mathbf{H}$ NMR (400 MHz, $\left.\mathbf{C D C l}_{3}\right): \delta 7.45(\mathrm{dd}, J=7.3,1.7 \mathrm{~Hz}, 1 \mathrm{H}), 7.19(\mathrm{~m}, 3 \mathrm{H}), 6.04(\mathrm{ddd}, J=17.2,10.3,5.8 \mathrm{~Hz}, 1 \mathrm{H}), 5.42(\mathrm{~d}$, 
$J=5.7 \mathrm{~Hz}, 1 \mathrm{H}), 5.32(\mathrm{dt}, J=17.1,1.5 \mathrm{~Hz}, 1 \mathrm{H}), 5.21(\mathrm{dt}, J=10.3,1.4 \mathrm{~Hz}, 1 \mathrm{H}), 2.36(\mathrm{~s}, 3 \mathrm{H}), 1.84(\mathrm{~s}, 1 \mathrm{H})$.

\section{(E)-3-Chloro-1-(o-tolyl)-1-propene (8b)}

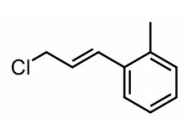

A flame-dried vial was charged with alcohol $\mathbf{S 3}$ (445 mg, $3.00 \mathrm{mmol}, 1.00$ equiv), then evacuated and backfilled with $\mathrm{N}_{2}$ three times. Anhydrous $\mathrm{CH}_{2} \mathrm{Cl}_{2}(6 \mathrm{~mL})$ was added via syringe and the resulting solution was cooled to $0{ }^{\circ} \mathrm{C}$. Neat, freshly distilled thionyl chloride $(1.1 \mathrm{~mL}, 15 \mathrm{mmol}, 5.0$ equiv) was then added dropwise via syringe to reaction flask. The reaction mixture was stirred at $0{ }^{\circ} \mathrm{C}$ for about 30 minutes, at which point the reaction was complete by TLC. The reaction mixture was then poured slowly into cold water $(10 \mathrm{~mL})$ and shaken vigorously until organic layer became clear. The layers were separated, then the aqueous layer was extracted with $\mathrm{CH}_{2} \mathrm{Cl}_{2}(3$ $\times 5 \mathrm{~mL}$ ). The combined organic layers were washed with saturated $\mathrm{NaHCO}_{3}(2 \times 5 \mathrm{~mL})$, dried over $\mathrm{MgSO}_{4}$, and concentrated in vacuo to afford a light brown oil that was unstable to silica gel. This material was pure by ${ }^{1} \mathrm{H}$ NMR and was used without purification. The ${ }^{1} \mathrm{H}$ NMR data match the literature. ${ }^{9}$ (420 mg, 84\%)

Physical appearance: Light brown oil

${ }^{1}$ H NMR (400 MHz, CDCl $\left.)_{3}\right): \delta 7.44(\mathrm{~m}, 1 \mathrm{H}), 7.17(\mathrm{~m}, 3 \mathrm{H}), 6.88(\mathrm{~d}, J=15.5 \mathrm{~Hz}, 1 \mathrm{H}), 6.21(\mathrm{dt}, J=15.5,7.2 \mathrm{~Hz}, 1 \mathrm{H}), 4.26$ (dd, $J=7.2,1.2 \mathrm{~Hz}, 2 \mathrm{H}), 2.36(\mathrm{~s}, 3 \mathrm{H})$.

\section{1-(m-Methoxyphenyl)-2-propen-1-ol (S4)}

A flame-dried flask under $\mathrm{N}_{2}$ was charged with freshly distilled $m$-anisaldehyde $(1.22 \mathrm{~mL}, 10.0 \mathrm{mmol}$,
1.00 equiv) and freshly distilled THF $(10 \mathrm{~mL})$ and the resulting solution was cooled to $0{ }^{\circ} \mathrm{C}$.
Vinylmagnesium bromide $(1.0 \mathrm{M}$ in THF; $10.5 \mathrm{~mL}, 10.5 \mathrm{mmol}, 1.05$ equiv) was added dropwise over a period of 10 minutes. The reaction was allowed to warm to room temperature as the ice bath expired, and after 1 hour was quenched with saturated $\mathrm{NH}_{4} \mathrm{Cl}(20 \mathrm{~mL})$. The layers were separated, then the aqueous phase was extracted with $\mathrm{Et}_{2} \mathrm{O}(3 \times$ $10 \mathrm{~mL})$. The combined organic layers were washed with saturated $\mathrm{NaCl}(30 \mathrm{~mL})$, dried over $\mathrm{MgSO}_{4}$, and concentrated in vacuo to afford a yellow oil. Purification by flash chromatography (50 g silica, 4:1 petroleum ether/EtOAc) afforded the title compound as a clear colorless oil. The ${ }^{1} \mathrm{H}$ NMR data match the literature. ${ }^{10}(1.199 \mathrm{~g}, 73 \%)$

Physical appearance: Clear colorless oil

TLC $R_{\mathbf{f}}$ (4:1 petroleum ether/EtOAc, UV): 0.27

${ }^{1}$ H NMR (300 MHz, CDCl $): \delta 7.29(\mathrm{~m}, 1 \mathrm{H}), 6.96(\mathrm{~m}, 2 \mathrm{H}), 6.84(\mathrm{~m}, 1 \mathrm{H}), 6.05(\mathrm{ddd}, J=16.4,10.1,6.0 \mathrm{~Hz}, 1 \mathrm{H}), 5.37$ (m, $1 \mathrm{H}), 5.21(\mathrm{~m}, 2 \mathrm{H}), 3.82(\mathrm{~s}, 3 \mathrm{H}), 1.93(\mathrm{~s}, 1 \mathrm{H})$.

\section{(E)-3-Chloro-1-(m-methoxyphenyl)-1-propene (8c)}

OMe A flame-dried vial was charged with alcohol S4 (493 mg, $3.00 \mathrm{mmol}, 1.00$ equiv), then evacuated and backfilled with $\mathrm{N}_{2}$ three times. Anhydrous $\mathrm{CH}_{2} \mathrm{Cl}_{2}(6 \mathrm{~mL})$ was added via syringe and the resulting solution was cooled to $0{ }^{\circ} \mathrm{C}$. Neat, freshly distilled thionyl chloride $(1.1 \mathrm{~mL}, 15 \mathrm{mmol}, 5.0$ equiv) was then added dropwise via syringe to reaction flask. The reaction mixture was stirred at $0{ }^{\circ} \mathrm{C}$ for about 30 minutes, at which point the reaction was complete by TLC. The reaction mixture was then poured slowly into cold water $(10 \mathrm{~mL})$ and shaken vigorously until organic layer became clear. The layers were separated, then the aqueous layer was extracted with $\mathrm{CH}_{2} \mathrm{Cl}_{2}(3 \times 5 \mathrm{~mL})$. The combined organic layers were washed with saturated $\mathrm{NaHCO}_{3}(2 \times 5 \mathrm{~mL})$, dried over $\mathrm{MgSO}_{4}$, and concentrated in vacuo to afford a light brown oil that was unstable to silica gel. This material was pure by ${ }^{1} \mathrm{H}$ NMR and was used without purification. The ${ }^{1} \mathrm{H}$ NMR data match the literature. ${ }^{6}$ (499 $\mathrm{mg}, 91 \%$ )

Physical appearance: Light brown oil

${ }^{1} \mathrm{H}$ NMR (400 MHz, CDCl $)$ ): $\delta 7.25(\mathrm{t}, J=7.9 \mathrm{~Hz}, 1 \mathrm{H}), 6.99(\mathrm{~d}, J=7.6 \mathrm{~Hz}, 1 \mathrm{H}), 6.92(\mathrm{~m}, 1 \mathrm{H}), 6.83(\mathrm{~m}, 1 \mathrm{H}), 6.63(\mathrm{~d}, J=$ 
$15.6 \mathrm{~Hz}, 1 \mathrm{H}), 6.31(\mathrm{dt}, J=15.6,7.2 \mathrm{~Hz}, 1 \mathrm{H}), 4.24(\mathrm{dd}, J=7.2,1.2 \mathrm{~Hz}, 2 \mathrm{H}), 3.82(\mathrm{~s}, 3 \mathrm{H})$.

\section{1-(4-Biphenylyl)-2-propen-1-ol (S5)}

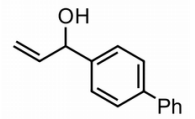

A flame-dried flask was charged with biphenyl-4-carboxaldehyde (1.822 g, $10.00 \mathrm{mmol}, 1.00$ equiv), sealed with a septum, then evacuated and backfilled with $\mathrm{N}_{2}$ three times. Freshly distilled THF $(10 \mathrm{~mL})$ was added via syringe, and the resulting solution was cooled to $0{ }^{\circ} \mathrm{C}$. Vinylmagnesium bromide (1.0 M in THF; 10.5

$\mathrm{mL}, 10.5 \mathrm{mmol}, 1.05$ equiv) was added dropwise along the side of the flask over a period of 10 minutes. The reaction was allowed to warm to room temperature as the ice bath expired, and after several hours was quenched with saturated $\mathrm{NH}_{4} \mathrm{Cl}$ $(20 \mathrm{~mL})$. The layers were separated, then the aqueous phase was extracted with $\mathrm{Et}_{2} \mathrm{O}(3 \times 10 \mathrm{~mL})$. The combined organic layers were washed with saturated $\mathrm{NaCl}(30 \mathrm{~mL})$, dried over $\mathrm{MgSO}_{4}$, and concentrated in vacuo to afford a yellow oil. Purification by flash chromatography (50 g silica, 4:1 petroleum ether/EtOAc) afforded the title compound as a white waxy solid. The ${ }^{1} \mathrm{H}$ NMR data match the literature. ${ }^{11}(1.489 \mathrm{~g}, 71 \%)$

Physical appearance: White solid

TLC $R_{\mathbf{f}}$ (4:1 petroleum ether/EtOAc, UV): 0.27

${ }^{1}$ H NMR (400 MHz, CDCl $): \delta 7.59(\mathrm{~m}, 4 \mathrm{H}), 7.45(\mathrm{~m}, 4 \mathrm{H}), 7.35(\mathrm{t}, J=7.3 \mathrm{~Hz}, 1 \mathrm{H}), 6.10(\mathrm{ddd}, J=16.6,10.1,6.0 \mathrm{~Hz}, 1 \mathrm{H})$, $5.40(\mathrm{~d}, J=17.4 \mathrm{~Hz}, 1 \mathrm{H}), 5.25(\mathrm{~m}, 2 \mathrm{H}), 1.94(\mathrm{~d}, J=3.4 \mathrm{~Hz}, 1 \mathrm{H})$.

\section{(E)-1-(4-Biphenylyl)-3-chloro-1-propene (8d)}

A flame-dried flask was charged with alcohol S5 (421 mg, $2.00 \mathrm{mmol}, 1.00$ equiv), then evacuated and backfilled with $\mathrm{N}_{2}$ three times. Anhydrous $\mathrm{CH}_{2} \mathrm{Cl}_{2}(8 \mathrm{~mL})$ was added via syringe and the resulting solution was cooled to $0{ }^{\circ} \mathrm{C}$. Neat thionyl chloride $(1.5 \mathrm{~mL}, 20 \mathrm{mmol}, 10$ equiv) was then added dropwise via syringe to reaction flask. The reaction mixture was stirred at $0{ }^{\circ} \mathrm{C}$ for one hour, at which point the reaction was complete by TLC. The reaction mixture was then poured slowly into ice water $(\sim 30 \mathrm{~mL})$ and shaken vigorously until organic layer became clear. The layers were separated, then the aqueous layer was extracted with $\mathrm{CH}_{2} \mathrm{Cl}_{2}(3 \times 10 \mathrm{~mL})$. The combined organic layers were washed with saturated $\mathrm{NaHCO}_{3}(2 \times 25 \mathrm{~mL})$, dried over $\mathrm{MgSO}_{4}$, and concentrated in vacuo to afford an off-white solid that was pure by ${ }^{1} \mathrm{H}$ NMR and was used without purification. (323 mg, 71\%)

Physical appearance: Off-white solid

Melting point: $108-114^{\circ} \mathrm{C}$

TLC $\mathbf{R}_{\mathbf{f}}$ (19:1 petroleum ether/DCM): 0.40

${ }^{1}$ H NMR (400 MHz, $\left.\mathbf{C D C l}_{3}\right): \delta 7.65-7.55(\mathrm{~m}, 4 \mathrm{H}), 7.50-7.42(\mathrm{~m}, 4 \mathrm{H}), 7.41-7.33(\mathrm{~m}, 1 \mathrm{H}), 6.71(\mathrm{~d}, \mathrm{~J}=15.5 \mathrm{~Hz}, 1 \mathrm{H})$, $6.38(\mathrm{dtd}, \mathrm{J}=15.5,7.3,2.0 \mathrm{~Hz}, 1 \mathrm{H}), 4.28(\mathrm{dd}, \mathrm{J}=7.2,1.3 \mathrm{~Hz}, 2 \mathrm{H})$.

${ }^{13}$ C NMR (101 MHz, CDCl $): \delta 141.1,140.6,135.0,134.0,128.9,127.6,127.4,127.3,127.1,125.1,45.7$.

FT-IR (thin film): 3508, 3371, 3035, 2851, 1913, 1644, 1602, 1485, 1445, 1408, 1348, 1248, 1160, 1074, 1005, 971, 909, $813,759,732,688,660 \mathrm{~cm}^{-1}$.

HRMS: Calculated for $\mathrm{C}_{15} \mathrm{H}_{13} \mathrm{Cl}[\mathrm{M}]^{+} 228.0706$, found 228.0706.

\section{1-(6-Methoxy-2-naphthyl)-2-propen-1-ol (S6)}

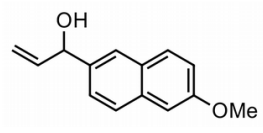

A flame-dried flask was charged with 6-methoxy-2-naphthaldehyde (1.862 g, $10.00 \mathrm{mmol}, 1.00$ equiv), sealed with a septum, then evacuated and backfilled with $\mathrm{N}_{2}$ three times. Freshly distilled THF $(10 \mathrm{~mL})$ was added via syringe, and the resulting solution was cooled to $0{ }^{\circ} \mathrm{C}$. Vinylmagnesium bromide $(1.0 \mathrm{M}$ in THF; $10.5 \mathrm{~mL}, 10.5 \mathrm{mmol}, 1.05$ equiv) was added dropwise over a period of 10 minutes. The reaction was allowed to warm to room temperature as the ice bath expired, and after 1 hour was quenched with saturated $\mathrm{NH}_{4} \mathrm{Cl}(20 \mathrm{~mL})$. The layers were separated, then the aqueous phase was extracted with $\mathrm{Et}_{2} \mathrm{O}(3 \times 10 \mathrm{~mL})$. The combined organic layers were washed 
with saturated $\mathrm{NaCl}(30 \mathrm{~mL})$, dried over $\mathrm{MgSO}_{4}$, and concentrated in vacuo to afford a light yellow semi-solid. Purification by flash chromatography ( $60 \mathrm{~g}$ silica, $4: 1$ petroleum ether/EtOAc) afforded the title compound as a white solid. The ${ }^{1} \mathrm{H}$ NMR data match the literature. ${ }^{12}(1.489 \mathrm{~g}, 71 \%)$

Physical appearance: White solid

TLC $\mathbf{R}_{\mathbf{f}}$ (3:1 petroleum ether/EtOAc, UV): 0.31

${ }^{1}$ H NMR (300 MHz, CDCl $)$ : $\delta 7.74(\mathrm{~m}, 3 \mathrm{H}), 7.46(\mathrm{dd}, J=8.4,1.8 \mathrm{~Hz}, 1 \mathrm{H}), 7.15(\mathrm{~d}, J=8.8 \mathrm{~Hz}, 2 \mathrm{H}), 6.13$ (ddd, $J=16.7$, $10.4,6.0 \mathrm{~Hz}, 1 \mathrm{H}), 5.41(\mathrm{~m}, 2 \mathrm{H}), 5.24(\mathrm{~d}, J=10.3 \mathrm{~Hz}, 1 \mathrm{H}), 3.93(\mathrm{~s}, 3 \mathrm{H}), 1.99(\mathrm{~d}, J=3.5 \mathrm{~Hz}, 1 \mathrm{H})$.

(E)-3-Chloro-1-(6-methoxy-2-naphthyl)-1-propene (8e)

An attempt to prepare the title compound using thionyl chloride resulted in partial decomposition, so an alternative protocol was used. A vial was charged with alcohol $\mathbf{S 6}(214 \mathrm{mg}, 1.00 \mathrm{mmol}, 1.00$ equiv) and bulk THF $(0.5 \mathrm{~mL})$, then concentrated aqueous $\mathrm{HCl}(83 \mu \mathrm{L}, 1.0 \mathrm{mmol}, 1.0$ equiv) was added dropwise with vigorous stirring at room temperature. After about 20 minutes, progress had ceased by TLC, so additional concentrated aqueous $\mathrm{HCl}(83 \mu \mathrm{L}, 1.0 \mathrm{mmol}, 1.0$ equiv) was added dropwise. After an additional 5 minutes, the starting material was consumed by TLC and the reaction was quenched with saturated $\mathrm{NaHCO}_{3}(1 \mathrm{~mL})$. The reaction was extracted with a mixture of $\mathrm{Et}_{2} \mathrm{O}$ and $\mathrm{EtOAc}(\sim 1 \mathrm{~mL})$, and the organic layer was passed through a plug of $\mathrm{MgSO}_{4}$ and concentrated in vacuo to afford a white solid that was unstable to silica gel. This material was pure by ${ }^{1} \mathrm{H}$ NMR and was used without purification. (199 mg, 86\%)

Physical appearance: White solid

Melting point: $91-94{ }^{\circ} \mathrm{C}$; decomposition at $139^{\circ} \mathrm{C}$

${ }^{1}$ H NMR (400 MHz, $\left.\mathbf{C D C l}_{3}\right): \delta 7.69(\mathrm{~m}, 3 \mathrm{H}), 7.55(\mathrm{dd}, J=8.5,1.8 \mathrm{~Hz}, 1 \mathrm{H}), 7.14(\mathrm{dd}, J=8.9,2.5 \mathrm{~Hz}, 1 \mathrm{H}), 7.11(\mathrm{~d}, J=2.5$ $\mathrm{Hz}, 1 \mathrm{H}), 6.78(\mathrm{~d}, J=15.6 \mathrm{~Hz}, 1 \mathrm{H}), 6.39(\mathrm{dt}, J=15.5,7.2 \mathrm{~Hz}, 1 \mathrm{H}), 4.29(\mathrm{dd}, J=7.3,1.1 \mathrm{~Hz}, 2 \mathrm{H}), 3.92(\mathrm{~s}, 3 \mathrm{H})$.

${ }^{13}$ C NMR (101 MHz, CDCl $)$ : $\delta 158.12(\mathrm{C}), 134.7(\mathrm{C}), 134.6(\mathrm{CH}), 131.4(\mathrm{C}), 129.7(\mathrm{CH}), 129.0(\mathrm{C}), 127.3(\mathrm{CH}), 127.0$ $(\mathrm{CH}), 124.3(\mathrm{CH}), 124.2(\mathrm{CH}), 119.3(\mathrm{CH}), 106.0(\mathrm{CH}), 55.5\left(\mathrm{CH}_{3}\right), 45.9\left(\mathrm{CH}_{2}\right)$.

FT-IR (thin film): 3036, 3000, 2940, 2846, 1626, 1600, 1504, 1482, 1439, 1414, 1390, 1344, 1267, 1242, 1198, 1163 , 1120, 1030, 967, 898, 867, 822, 804, 707, $652 \mathrm{~cm}^{-1}$.

HRMS (EI): Calculated for $\mathrm{C}_{14} \mathrm{H}_{13} \mathrm{ClO}[\mathrm{M}]^{+} 232.0655$, found 232.0665 .

\section{5-[(E)-4-Bromo-2-butenyl]-2,2,5-trimethyl-1,3-dioxane-4,6-dione (8h)}

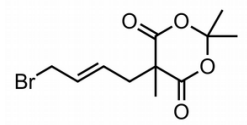

A vial was charged with methyl Meldrum's acid (395 mg, $2.50 \mathrm{mmol}, 1.00$ equiv), (E)-1,4-dibromo-2butene (1.070 g, $5.00 \mathrm{mmol}, 2.00$ equiv), $\mathrm{K}_{2} \mathrm{CO}_{3}$ ( $380 \mathrm{mg}, 2.75 \mathrm{mmol}, 1.10$ equiv), and bulk MeCN (2.5 $\mathrm{mL})$. The vial was capped and the resulting suspension was stirred overnight, at which point it was diluted with EtOAc and filtered through Celite. The filtrate was concentrated in vacuo and purified by flash chromatography (17 g silica, 4:1 petroleum ether/EtOAc) to afford the title compound as a white solid. (589 mg, 81\%)

Physical appearance: White solid

Melting point: $46-51^{\circ} \mathrm{C}$

TLC $\mathbf{R}_{\mathrm{f}}$ (13:1 petroleum ether/Et $\left.\mathbf{t}_{2} \mathrm{O}, \mathrm{KMnO}_{4}\right): 0.33$

${ }^{1}$ H NMR (400 MHz, CDCl $): \delta 5.86(\mathrm{~m}, 1 \mathrm{H}), 5.64(\mathrm{~m}, 1 \mathrm{H}), 3.87(\mathrm{~d}, J=7.3 \mathrm{~Hz}, 2 \mathrm{H}), 2.76(\mathrm{~d}, J=7.6 \mathrm{~Hz}, 2 \mathrm{H}), 1.73(\mathrm{~s}, 6 \mathrm{H})$, $1.64(\mathrm{~s}, 3 \mathrm{H})$.

${ }^{813}$ C NMR (101 MHz, CDCl3): $\delta 169.8(\mathrm{C}), 132.7(\mathrm{CH}), 128.0(\mathrm{CH}), 105.4(\mathrm{C}), 50.0(\mathrm{C}), 42.1\left(\mathrm{CH}_{2}\right), 31.5\left(\mathrm{CH}_{2}\right), 29.7$ $\left(\mathrm{CH}_{3}\right), 29.0\left(\mathrm{CH}_{3}\right), 24.4\left(\mathrm{CH}_{3}\right)$.

FT-IR (thin film): 2997, 2942, 1778, 1742, 1455, 1382, 1314, 1271, 1205, 1175, 1147, 1059, 975, 932, $688 \mathrm{~cm}^{-1}$. 
HRMS (EI): No molecular ion signal was observed, but fragmentation peaks for $\mathrm{C}_{11} \mathrm{H}_{15} \mathrm{BrO}_{4}[\mathrm{M}-\mathrm{Br}]^{+}$(calculated 211.0966, found 211.1) and $\left[\mathrm{M}-\mathrm{CH}_{3}\right]^{+}$(calculated 274.9914 and 276.9893, found 275.0 and 277.0) were prominent.

\section{(E)-4-Bromo-2-butenyl 2,2-dimethylpropionate (8i)}

حOPv A vial was charged with pivalic acid (255 mg, $2.50 \mathrm{mmol}, 1.00$ equiv), (E)-1,4-dibromo-2-butene (1.070 g, $5.00 \mathrm{mmol}, 2.00$ equiv), $\mathrm{K}_{2} \mathrm{CO}_{3}(380 \mathrm{mg}, 2.75 \mathrm{mmol}, 1.10$ equiv), and bulk $\mathrm{MeCN}$ (2.5 mL). The vial was capped and the resulting suspension was stirred for 2 days, at which point it was diluted with EtOAc and filtered through Celite. The filtrate was concentrated in vacuo and purified by flash chromatography ( $30 \mathrm{~g}$ silica, 15:1 petroleum ether/Et ${ }_{2} \mathrm{O}$ ) to afford the title compound as a clear colorless oil. (463 mg, 79\%)

Physical appearance: Clear colorless oil

TLC $\mathbf{R}_{\mathbf{f}}$ (13:1 petroleum ether/Et $\left.\mathbf{t}_{2} \mathbf{O}, \mathrm{KMnO}_{4}\right)$ : 0.33

${ }^{1}$ H NMR (400 MHz, CDCl $)$ ): $\delta 5.95(\mathrm{~m}, 1 \mathrm{H}), 5.86(\mathrm{~m}, 1 \mathrm{H}), 4.58(\mathrm{dd}, J=5.4,1.2 \mathrm{~Hz}, 2 \mathrm{H}), 3.96(\mathrm{dd}, J=7.3,0.9 \mathrm{~Hz}, 2 \mathrm{H})$, $1.22(\mathrm{~s}, 9 \mathrm{H})$.

${ }^{13}$ C NMR (101 MHz, CDCl $)$ : $\delta 178.3(\mathrm{C}), 129.5(\mathrm{CH}), 129.5(\mathrm{CH}), 63.5\left(\mathrm{CH}_{2}\right), 39.0(\mathrm{C}), 31.7\left(\mathrm{CH}_{2}\right), 27.4\left(\mathrm{CH}_{3}\right)$. FT-IR (thin film): 2972, 2874, 1730, 1480, 1459, 1398, 1368, 1281, 1207, 1135, 1091, 1057, 1032, 966, 859, $771 \mathrm{~cm}^{-1}$. HRMS (EI): Calculated for $\mathrm{C}_{9} \mathrm{H}_{15} \mathrm{BrO}_{2}[\mathrm{M}-\mathrm{Br}]^{+}$155.10721, found 155.10691 .

\section{2-[(E)-4-Bromo-2-butenyl]-1,3-isoindolinedione (8j)}

A vial was charged with potassium phthalimide (926 mg, $5.00 \mathrm{mmol}, 1.00$ equiv), (E)-1,4-dibromo-2butene $(2.139 \mathrm{~g}, 10.00 \mathrm{mmol}, 2.00$ equiv), and bulk DMF $(5 \mathrm{~mL})$. The vial was capped, placed in a preheated $50{ }^{\circ} \mathrm{C}$ bath, and stirred overnight. After cooling to room temperature, it was poured into water $(50 \mathrm{~mL})$ with stirring, leading to the formation of a white precipitate which was collected by vacuum filtration. Purification of this material by flash chromatography ( $30 \mathrm{~g}$ silica, $4: 1 \rightarrow 3: 1$ petroleum ether/EtOAc) afforded the title compound as a white crystalline solid. The ${ }^{1} \mathrm{H}$ NMR data match the literature. ${ }^{13}$ (933 mg, 67\%)

Physical appearance: White solid

${ }^{1} \mathbf{H}$ NMR (400 MHz, $\left.\mathbf{C D C l}_{3}\right): \delta 7.86(\mathrm{~m}, 2 \mathrm{H}), 7.73(\mathrm{~m}, 2 \mathrm{H}), 5.95(\mathrm{dtt}, J=16.2,7.1,1.2 \mathrm{~Hz}, 1 \mathrm{H}), 5.84(\mathrm{dtt}, J=15.2,6.0,0.8$ $\mathrm{Hz}, 1 \mathrm{H}), 4.31$ (ddd, $J=6.0,1.3,0.7 \mathrm{~Hz}, 2 \mathrm{H}), 3.91(\mathrm{dq}, J=7.3,0.8 \mathrm{~Hz}, 2 \mathrm{H})$. 


\section{Ru-Catalyzed Asymmetric Allylic Alkylation Reactions}

\section{General Procedure A}

One vial was charged with $\mathrm{CpRu}(\mathrm{MeCN})_{3} \mathrm{PF}_{6}(1.1 \mathrm{mg}, 0.0025 \mathrm{mmol}, 0.005$ equiv), ligand $\mathbf{L 1}$ (2.9 mg, $0.0030 \mathrm{mmol}$, 0.0060 equiv), and bulk 3-pentanone $(50 \mu \mathrm{L})$. Another vial was charged with isatin 7 ( $0.50 \mathrm{mmol}, 1.00$ equiv) and bulk 3pentanone $(50 \mu \mathrm{L})$. Next, DIPEA ( $89 \mu \mathrm{L}, 0.51 \mathrm{mmol}, 1.02$ equiv) was added to the vial containing the isatin, followed by the premixed catalyst solution. Finally, the allylic halide $8(0.50 \mathrm{mmol}, 1.00$ equiv $)$ was added. The reaction vial was capped under ambient atmosphere and the reaction was stirred overnight at $40{ }^{\circ} \mathrm{C}$. After assessing branched/linear selectivity by crude ${ }^{1} \mathrm{H}$ NMR, the reaction was concentrated in vacuo and purified by flash chromatography using the specified conditions to afford the allylated product 9.

\section{General Procedure B}

One vial was charged with $\mathrm{CpRu}(\mathrm{MeCN})_{3} \mathrm{PF}_{6}(1.1 \mathrm{mg}, 0.0025 \mathrm{mmol}, 0.005$ equiv), ligand $\mathbf{L 1}$ (2.9 $\mathrm{mg}, 0.0030 \mathrm{mmol}$, 0.0060 equiv), and bulk dimethyl carbonate $(50 \mu \mathrm{L})$. Another vial was charged with isatin 7 (0.50 mmol, 1.00 equiv) and bulk dimethyl carbonate $(50 \mu \mathrm{L})$. Next, DIPEA ( $89 \mu \mathrm{L}, 0.51 \mathrm{mmol}, 1.02$ equiv) was added to the vial containing the isatin, followed by the premixed catalyst solution. Finally, the allylic halide $8(0.50 \mathrm{mmol}, 1.00$ equiv) was added. The reaction vial was capped under ambient atmosphere and the reaction was stirred overnight at $40{ }^{\circ} \mathrm{C}$. After assessing branched/linear selectivity by crude ${ }^{1} \mathrm{H}$ NMR, the reaction was concentrated in vacuo and purified by flash chromatography using the specified conditions to afford the allylated product 9.

\section{Detailed Millimole-Scale Procedure}

One vial was charged with $\mathrm{CpRu}(\mathrm{MeCN})_{3} \mathrm{PF}_{6}(4.3 \mathrm{mg}, 0.010 \mathrm{mmol}, 0.0050$ equiv), ligand $\mathbf{L 1}$ (11.5 mg, I $=0.0120 \mathrm{mmol}, 0.00600$ equiv), and bulk 3-pentanone $(0.2 \mathrm{~mL})$. Another vial was charged with 5-chloroisatin $=\gamma_{\mathrm{Ph}} 7 \mathbf{d}(363 \mathrm{mg}, 2.00 \mathrm{mmol}, 1.00$ equiv) and bulk 3-pentanone $(0.2 \mathrm{~mL})$. Next, DIPEA (0.36 mL, $2.04 \mathrm{mmol}, 1.02$ equiv) was added to the vial containing the isatin, followed by the premixed catalyst solution. Finally, cinnamyl chloride 8a (279 $\mu \mathrm{L}, 2.00 \mathrm{mmol}, 1.00$ equiv). The reaction vial was capped under ambient atmosphere and the reaction was stirred overnight at $40{ }^{\circ} \mathrm{C}$. Crude ${ }^{1} \mathrm{H}$ NMR showed 11:1 branched/linear selectivity. Purification by flash chromatography $(25 \mathrm{~g}$ silica, 8:1 $\rightarrow$ 4:1 petroleum ether/EtOAc) afforded 9da as a bright orange solid that contained roughly 1 equivalent of $\mathrm{H}_{2} \mathrm{O}$ by ${ }^{1} \mathrm{H} \mathrm{NMR}$, but was otherwise pure. This material was dissolved in $\mathrm{CHCl}_{3}$, dried over $\mathrm{MgSO}_{4}$, filtered, and concentrated in vacuo to afford $9 \mathrm{da}$ as an orange solid. (437 mg, 73\%, 90:10 er) The spectral data for this compound are reported on page S13. 


\section{1-[(R)-1-Phenyl-2-propenyl]-2,3-indolinedione (9aa)}

The compound was prepared according to general procedure B using isatin $7 \mathbf{a}(73.6 \mathrm{mg}, 0.50 \mathrm{mmol}, 1.00$ equiv) and cinnamyl chloride 8a (70 $\mu \mathrm{L}, 0.50 \mathrm{mmol}, 1.00$ equiv). Crude ${ }^{1} \mathrm{H}$ NMR showed $>20: 1 \mathrm{branched} /$ linear selectivity. Purification by flash chromatography (silica, 15:1 to 7:1 petroleum ether/EtOAc) afforded 9aa as a bright orange solid. (120 mg, 91\%)

Physical appearance: Bright orange solid

Melting point: Changes appearance at $55^{\circ} \mathrm{C}$; completely melts by $129^{\circ} \mathrm{C}$

TLC $\mathbf{R}_{\mathbf{f}}$ (5:1 petroleum ether/EtOAc): 0.40

${ }^{1}$ H NMR (300 MHz, CDCl $)$ : $\delta 7.62(\mathrm{dd}, J=7.4,1.4 \mathrm{~Hz}, 1 \mathrm{H}), 7.37(\mathrm{~m}, 6 \mathrm{H}), 7.06(\mathrm{td}, J=7.5,0.8 \mathrm{~Hz}, 1 \mathrm{H}), 6.60(\mathrm{~d}, J=8.0$ $\mathrm{Hz}, 1 \mathrm{H}), 6.42$ (ddd, $J=16.9,10.3,6.7 \mathrm{~Hz}, 1 \mathrm{H}), 6.27$ (d, $J=6.6 \mathrm{~Hz}, 1 \mathrm{H}), 5.47$ (m, 2H).

${ }^{13}$ C NMR (101 MHz, $\left.\mathbf{C D C l}_{3}\right): \delta 183.3,158.2,149.9,138.0,136.3,132.0,129.1,128.3,127.3,125.5,123.7,120.7,118.1$, 113.3, 57.1.

FT-IR (thin film): 3063, 1738, 1610, 1494, 1467, 1416, 1347, 1302, 1184, 1158, 1095, 988, 941, 817, 755, $732 \mathrm{~cm}^{-1}$. HRMS: Calculated for $\mathrm{C}_{17} \mathrm{H}_{13} \mathrm{NO}_{2}[\mathrm{M}+\mathrm{H}]^{+}$264.1025, found 264.1023.

HPLC: 99:1 er (Daicel CHIRALPAK IA, 97:3 heptane $/ 2 \mathrm{PrOH}, 0.8 \mathrm{~mL} / \mathrm{min} ; T_{\text {major }}=17.3 \mathrm{~min}, T_{\text {minor }}=15.1 \mathrm{~min}$ ) $[\alpha]_{\mathbf{D}}{ }^{23}:+25.0^{\circ}\left(c=0.51, \mathrm{CHCl}_{3}\right)$
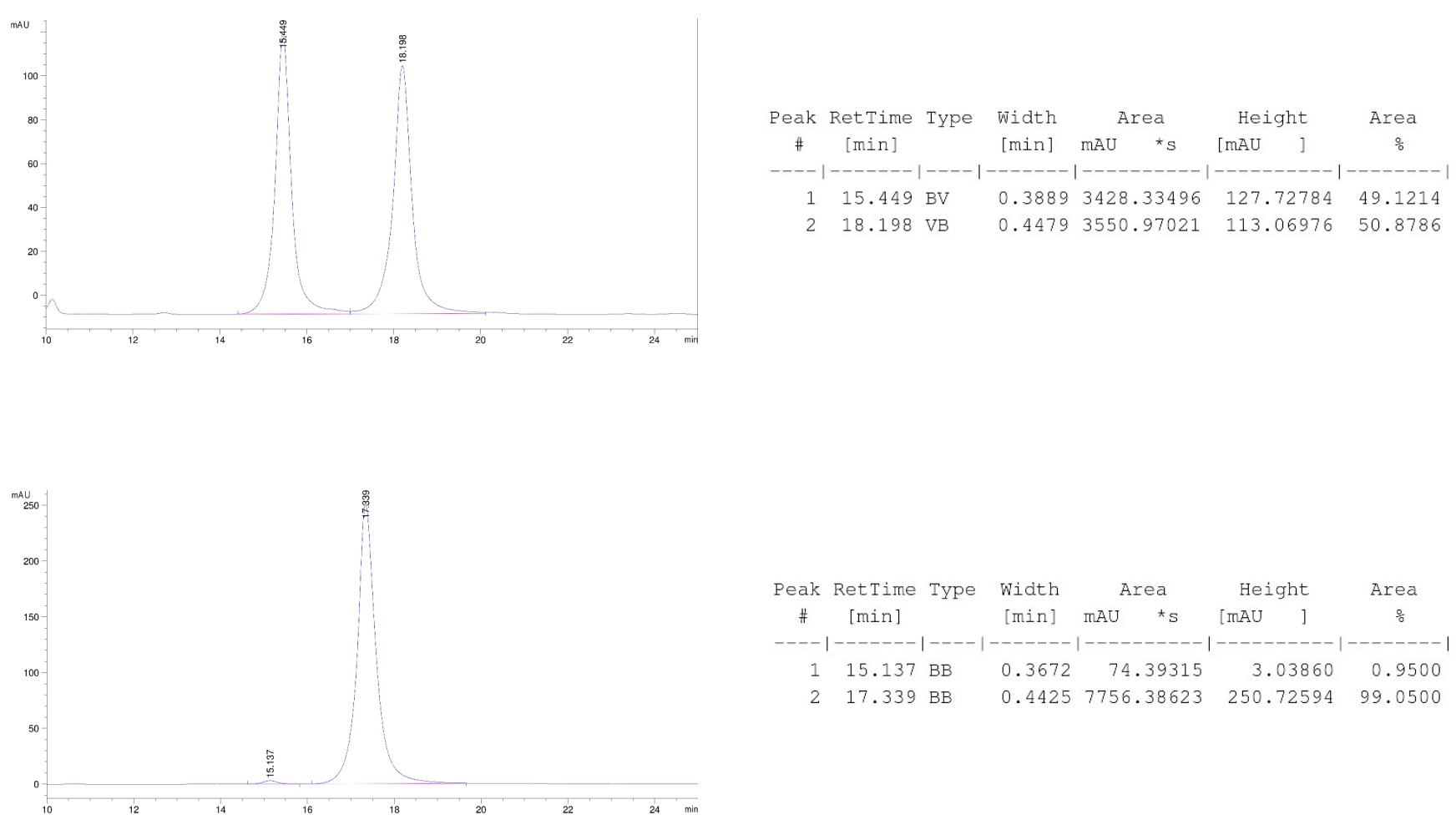


\section{1-[(R)-1-Phenyl-2-propenyl]-4-bromo-2,3-indolinedione (9ba)}

The compound was prepared according to general procedure A using 4-bromoisatin $7 \mathbf{b}(113.0 \mathrm{mg}, 0.50 \mathrm{mmol}$,
1.00 equiv) and cinnamyl chloride 8a (70 $\mu \mathrm{L}, 0.50 \mathrm{mmol}, 1.00$ equiv). Crude ${ }^{1} \mathrm{H}$ NMR showed $>20: 1$
branched/linear selectivity. Purification by flash chromatography (silica, 10:1 to $7: 1$ petroleum ether/EtOAc)
afforded $9 \mathbf{b a}$ as a bright orange solid. (141 mg, 82\%)

Physical appearance: Bright orange solid

Melting point: $132-134{ }^{\circ} \mathrm{C}$

TLC $R_{\mathbf{f}}$ (7:1 petroleum ether/EtOAc): 0.45

${ }^{1}$ H NMR (300 MHz, $\left.\mathbf{C D C l}_{3}\right): \delta 7.36(\mathrm{~m}, 5 \mathrm{H}), 7.17(\mathrm{~m}, 2 \mathrm{H}), 6.55(\mathrm{dd}, J=6.5,2.2 \mathrm{~Hz}, 1 \mathrm{H}), 6.40$ (ddd, $J=16.8,10.1,6.5$ $\mathrm{Hz}, 1 \mathrm{H}), 6.29(\mathrm{~d}, J=6.8 \mathrm{~Hz}, 1 \mathrm{H}), 5.51(\mathrm{dt}, J=10.3,0.9 \mathrm{~Hz}, 1 \mathrm{H}), 5.44(\mathrm{dt}, J=16.8,1.0 \mathrm{~Hz}, 2 \mathrm{H})$.

${ }^{13}$ C NMR (101 MHz, $\left.\mathbf{C D C l}_{3}\right): \delta 180.6,157.3,151.4,138.0,136.0,131.6,129.1,129.1,128.4,127.2,121.7,120.9,116.8$, 112.0, 57.2.

FT-IR (thin film): 2919, 2851, 1740, 1598, 1493, 1468, 1442, 1371, 1328, 1250, 1166, 1145, 1046, 993, 943, $779 \mathrm{~cm}^{-1}$.

HRMS: Calculated for $\mathrm{C}_{17} \mathrm{H}_{12} \mathrm{BrNO}_{2}[\mathrm{M}+\mathrm{H}]^{+} 342.0130$, found 342.0120 .

HPLC: $93: 7$ er (Daicel CHIRALPAK IA, 97:3 heptane $/ 2 \mathrm{PrOH}, 0.8 \mathrm{~mL} / \mathrm{min} ; T_{\text {major }}=19.5 \mathrm{~min}, T_{\text {minor }}=16.9 \mathrm{~min}$ ) $[\alpha]_{\mathbf{D}}{ }^{23}:+9.9^{\circ}\left(c=0.24, \mathrm{CHCl}_{3}\right)$
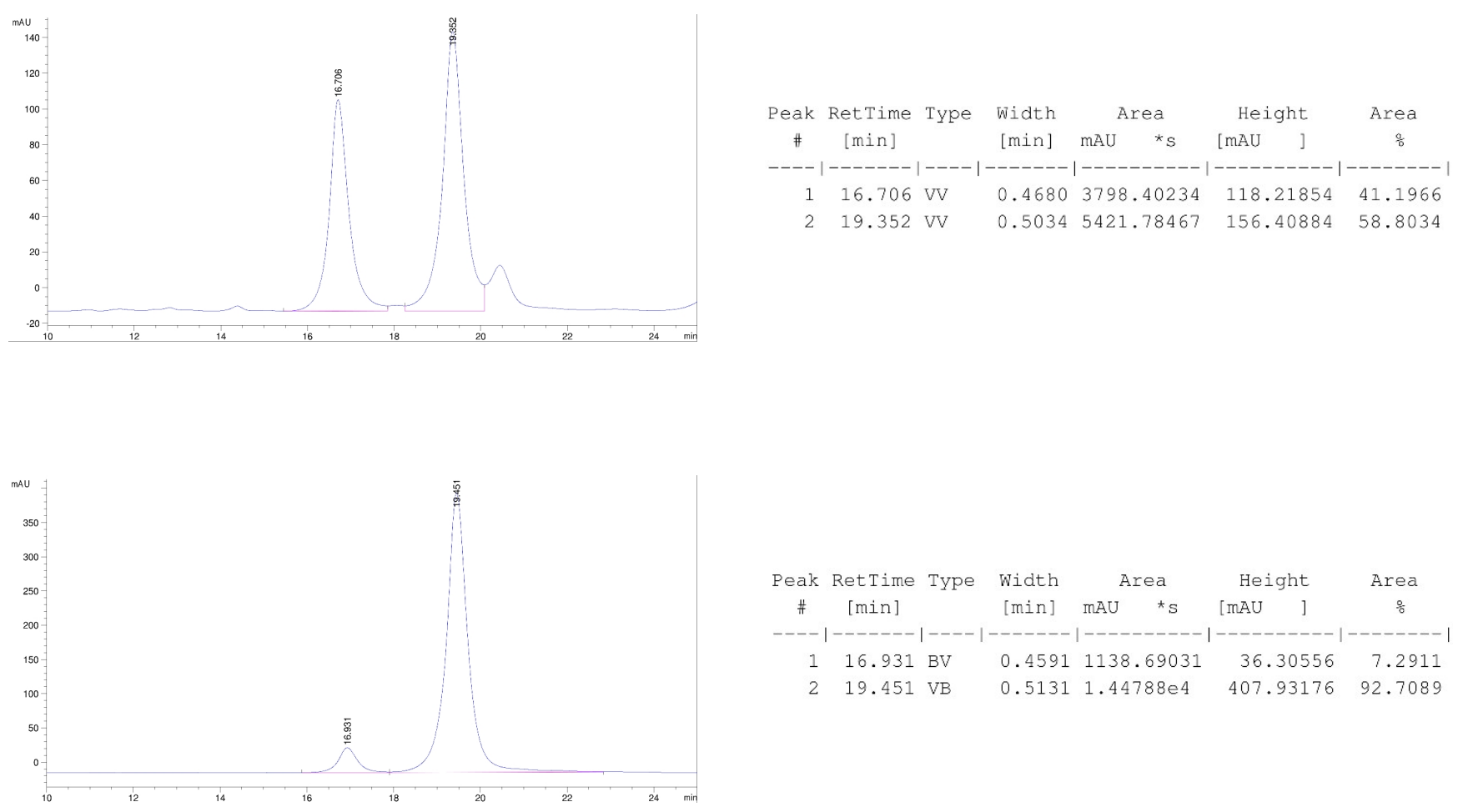


\section{1-[(R)-1-Phenyl-2-propenyl]-5-fluoro-2,3-indolinedione (9ca)}

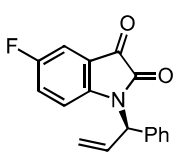

The title compound was prepared according to general procedure B using 5-fluoroisatin 7c $(82.6 \mathrm{mg}, 0.500$ mmol, 1.00 equiv) and cinnamyl chloride $8 \mathbf{a}\left(70 \mu \mathrm{L}, 0.50 \mathrm{mmol}, 1.00\right.$ equiv). Crude ${ }^{1} \mathrm{H}$ NMR showed 11:1 branched/linear selectivity. Purification by flash chromatography (7 $\mathrm{g}$ silica, 7:1 petroleum ether/EtOAc) afforded 9 ca as a bright orange-red solid. (71 mg, 50\%)

Physical appearance: Bright orange-red solid

Melting point: $78-80^{\circ} \mathrm{C}$

TLC $\mathbf{R}_{\mathbf{f}}$ (7:1 petroleum ether/EtOAc): 0.36

${ }^{1} \mathbf{H}$ NMR $\left(400 \mathrm{MHz}, \mathbf{C D C l}_{3}\right): \delta 7.34(\mathrm{~m}, 6 \mathrm{H}), 7.08(\mathrm{td}, J=8.7,2.7 \mathrm{~Hz}, 1 \mathrm{H}), 6.56(\mathrm{dd}, J=8.8,3.7 \mathrm{~Hz}, 1 \mathrm{H}), 6.40(\mathrm{ddd}, J=$ $16.9,10.3,6.6 \mathrm{~Hz}, 1 \mathrm{H}), 6.27(\mathrm{~d}, J=6.6 \mathrm{~Hz}, 1 \mathrm{H}), 5.51(\mathrm{dt}, J=10.3,1.1 \mathrm{~Hz}, 1 \mathrm{H}), 5.44(\mathrm{dd}, J=17.0,1.2 \mathrm{~Hz}, 1 \mathrm{H})$.

${ }^{13}$ C NMR (101 MHz, $\left.\mathbf{C D C l}_{3}\right): \delta$ 182.7, $159.2(\mathrm{~d}, J=236.3 \mathrm{~Hz}), 157.9,145.9,136.0,131.6,129.2,128.4,127.2,124.4(\mathrm{~d}, J$ $=23.9 \mathrm{~Hz}), 120.8,118.8(\mathrm{~d}, J=7.0 \mathrm{~Hz}), 114.5(\mathrm{~d}, J=6.9 \mathrm{~Hz}), 112.4(\mathrm{~d}, J=24.1 \mathrm{~Hz}), 57.1$.

${ }^{19}$ F NMR (376 MHz, $\left.\mathbf{C D C l}_{3}\right): \delta-118.73(\mathrm{ddd}, J=9.1,7.1,3.9 \mathrm{~Hz})$.

FT-IR (thin film): 3063, 2921, 1743, 1621, 1483, 1456, 1417, 1370, 1325, 1299, 1266, 1235, 1180, 1137, 1078, 1031, $1004,936,887,826,788,769,725,700,618 \mathrm{~cm}^{-1}$.

HRMS: Calculated for $\mathrm{C}_{17} \mathrm{H}_{12} \mathrm{FNO}_{2}[\mathrm{M}+\mathrm{H}]^{+}$282.0930, found 282.0925 .

HPLC: 86:14 er (Daicel CHIRALPAK AD-H, 90:10 heptane $/ \mathrm{PrOH}, 1.0 \mathrm{~mL} / \mathrm{min} ; T_{\text {major }}=15.1 \mathrm{~min}, T_{\text {minor }}=12.8 \mathrm{~min}$ ) $[\alpha]_{\mathrm{D}}{ }^{24}:+56.4^{\circ}\left(c=1.5, \mathrm{CHCl}_{3}\right)$

$-\frac{\mathrm{g}}{\underline{x}}$
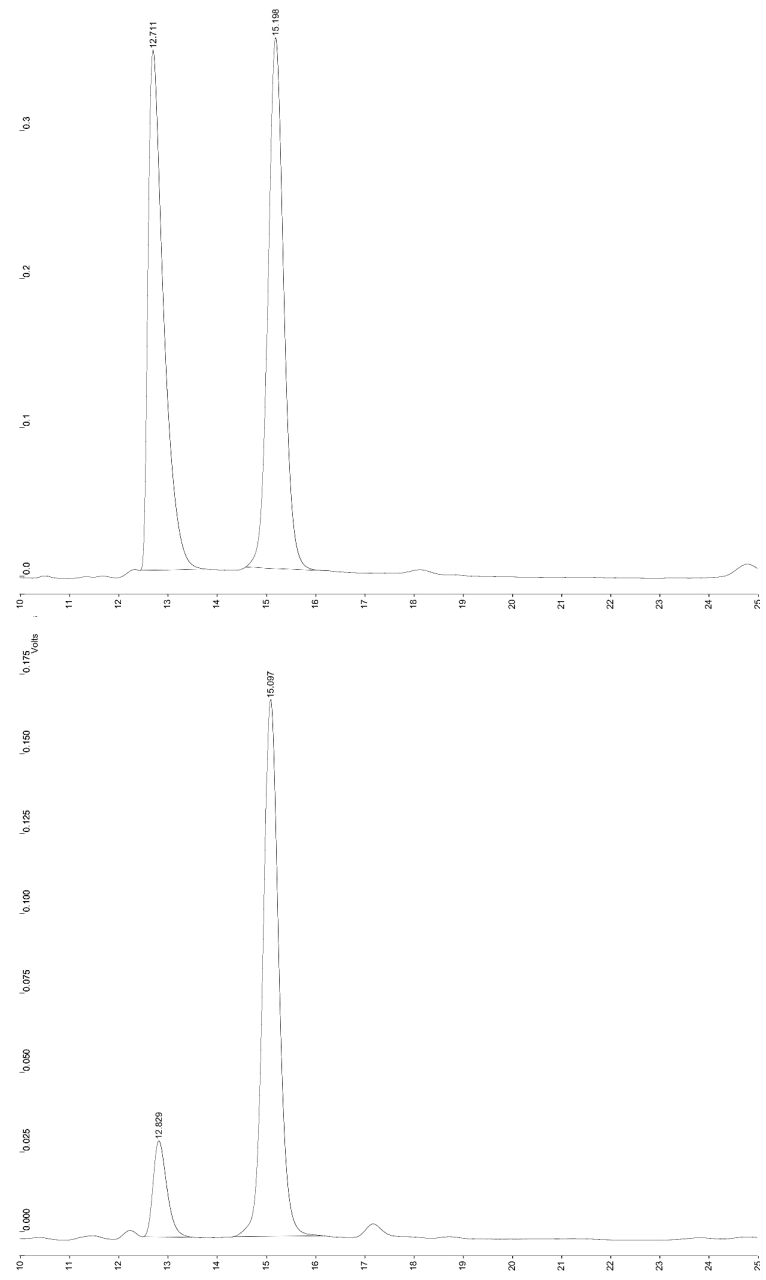

응

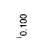

参

:

:

8
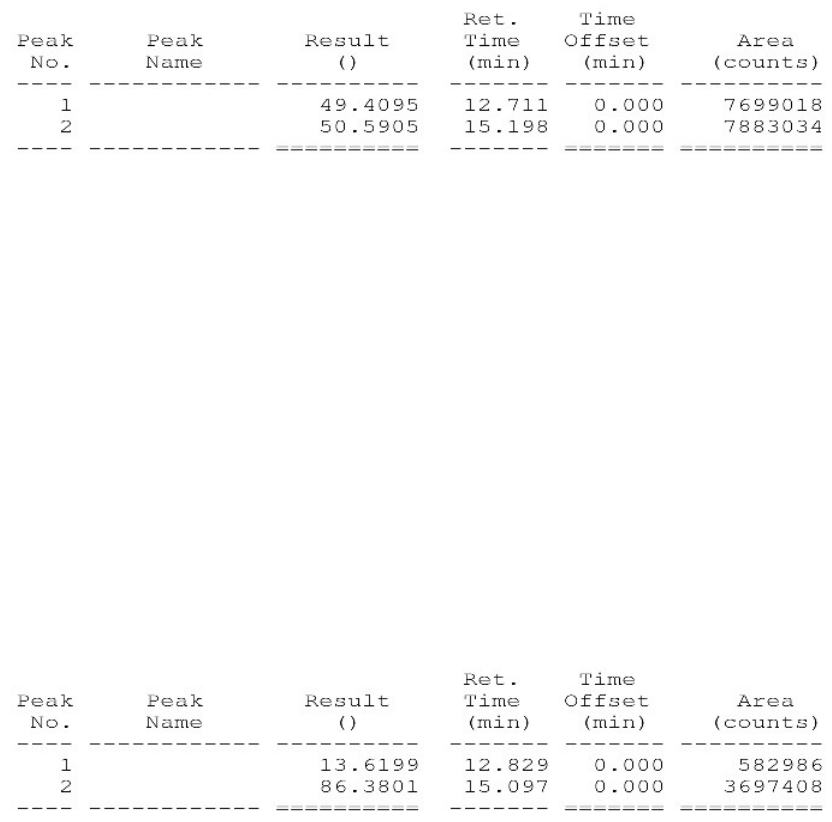

$\begin{array}{lrr}\begin{array}{l}\text { Ret. } \\ \text { Time } \\ \text { (min) }\end{array} & \begin{array}{c}\text { Time } \\ \text { Offset } \\ \text { (min) }\end{array} & \begin{array}{c}\text { Area } \\ \text { (counts) }\end{array} \\ ------ & ------ & -------- \\ 12.829 & 0.000 & 582986 \\ 15.097 & 0.000 & 3697408\end{array}$




\section{1-[(R)-1-Phenyl-2-propenyl]-5-chloro-2,3-indolinedione (9da)}

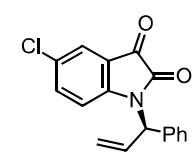

The compound was prepared according to general procedure A using 5-chloroisatin 7d $(90.8 \mathrm{mg}, 0.50 \mathrm{mmol}$, 1.00 equiv) and cinnamyl chloride 8a (70 $\mu \mathrm{L}, 0.50 \mathrm{mmol}, 1.00$ equiv). Crude ${ }^{1} \mathrm{H}$ NMR showed $>20: 1$ branched/linear selectivity. Purification by flash chromatography (silica, 10:1 to 7:1 petroleum ether/EtOAc) afforded 9da as a bright orange solid. (93 mg, 64\%)

Physical appearance: Bright orange solid

Melting point: Changes appearance at $107^{\circ} \mathrm{C}$; completely melts by $138^{\circ} \mathrm{C}$

TLC $R_{\mathbf{f}}$ (7:1 petroleum ether/EtOAc): 0.35

${ }^{1}$ H NMR (300 MHz, $\left.\mathbf{C D C l}_{3}\right): \delta 7.55(\mathrm{~d}, J=2.3 \mathrm{~Hz}, 1 \mathrm{H}), 7.35(\mathrm{~m}, 6 \mathrm{H}), 6.57(\mathrm{~d}, J=8.5 \mathrm{~Hz}, 1 \mathrm{H}), 6.40$ (ddd, $J=16.9,10.3$, $6.5 \mathrm{~Hz}, 1 \mathrm{H}), 6.26(\mathrm{~d}, J=6.6 \mathrm{~Hz}, 1 \mathrm{H}), 5.51(\mathrm{dt}, J=10.3,1.1 \mathrm{~Hz}, 1 \mathrm{H}), 5.43(\mathrm{~m}, 1 \mathrm{H})$.

${ }^{13}$ C NMR (101 MHz, CDCl $): \delta 182.4(\mathrm{C}), 157.7$ (C), 148.2 (C), $137.4(\mathrm{CH}), 136.0(\mathrm{C}), 131.6(\mathrm{CH}), 129.7(\mathrm{C}), 129.2$ $(\mathrm{CH}), 128.5(\mathrm{CH}), 127.3(\mathrm{CH}), 125.4(\mathrm{CH}), 120.9\left(\mathrm{CH}_{2}\right), 119.0(\mathrm{C}), 114.6(\mathrm{CH}), 57.2(\mathrm{CH})$.

FT-IR (thin film): 3085, 3033, 2920, 1744, 1609, 1494, 1468, 1370, 1322, 1288, 1260, 1183, 1128, 1072, 1031, 999, 940 , $910,824,777,742,699 \mathrm{~cm}^{-1}$.

HRMS: Calculated for $\mathrm{C}_{17} \mathrm{H}_{12} \mathrm{ClNO}_{2}[\mathrm{M}+\mathrm{H}]^{+} 298.0635$, found 298.0626.

HPLC: 98.5:1.5 er (Daicel CHIRALPAK IA, 90:10 heptane/EtOAc, $0.6 \mathrm{~mL} / \mathrm{min} ; T_{\text {major }}=21.8 \mathrm{~min}, T_{\text {minor }}=20.1 \mathrm{~min}$ ) $[\alpha]_{\mathbf{D}}{ }^{24}:+65.0^{\circ}\left(c=1.2, \mathrm{CHCl}_{3}\right)$
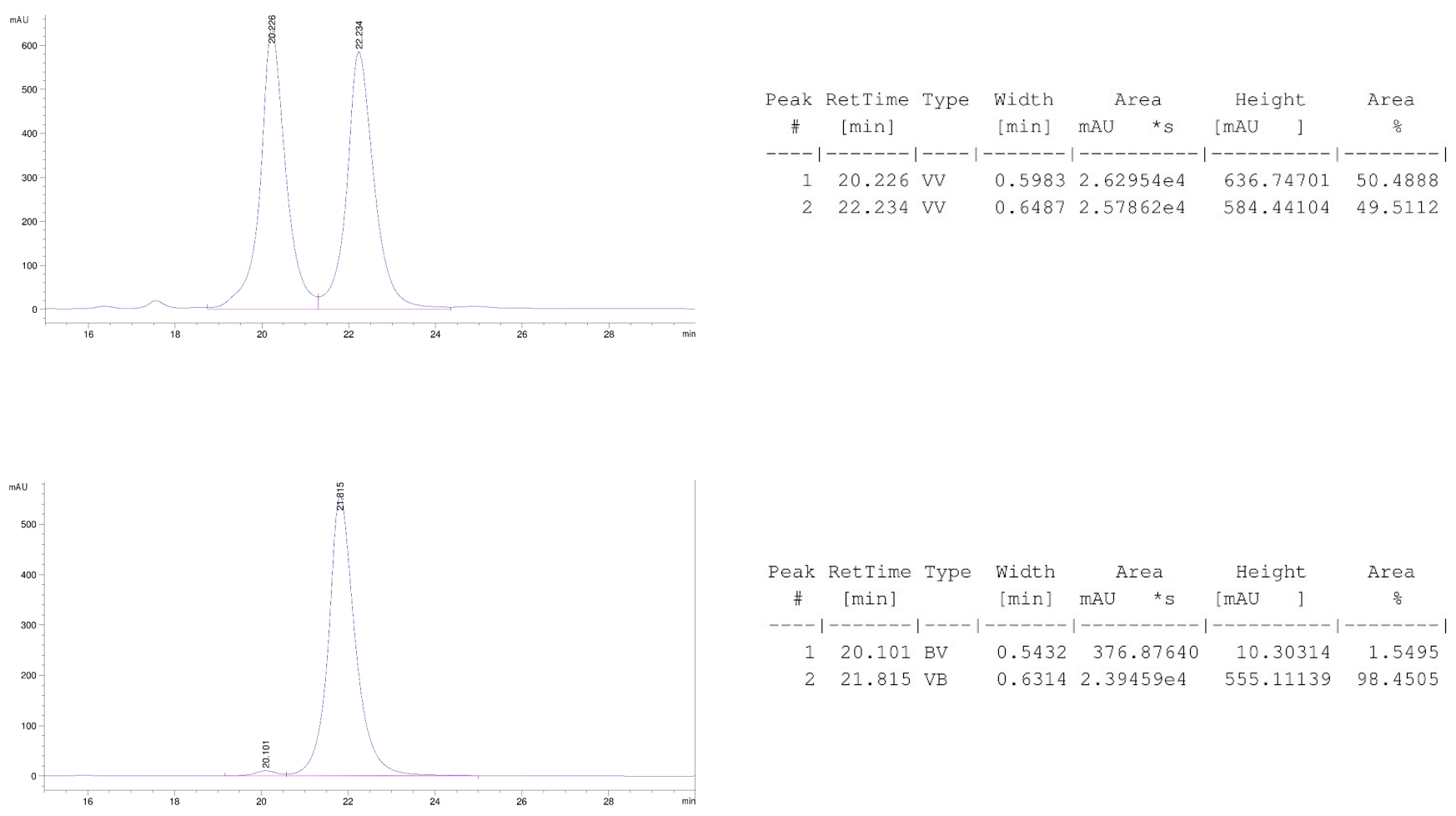


\section{1-[(R)-1-Phenyl-2-propenyl]-5-iodo-2,3-indolinedione (9ea)}

The title compound was prepared according to general procedure B using 5-iodoisatin 7 e (136.5 mg, 0.500 mmol, 1.00 equiv) and cinnamyl chloride $8 \mathbf{a}\left(70 \mu \mathrm{L}, 0.50 \mathrm{mmol}, 1.00\right.$ equiv). Crude ${ }^{1} \mathrm{H}$ NMR showed $>20: 1$ branched/linear selectivity. Purification by flash chromatography (silica, 10:1 to 7:1 petroleum ether/EtOAc) afforded 9ea as a bright orange solid. (132 $\mathrm{mg}, 68 \%$ )

Physical appearance: Bright orange solid

Melting point: Changes appearance at $98^{\circ} \mathrm{C}$; melts $110-115^{\circ} \mathrm{C}$

TLC $\mathbf{R}_{\mathbf{f}}$ (7:1 petroleum ether/EtOAc): 0.40

${ }^{1} \mathbf{H}$ NMR (300 MHz, $\left.\mathbf{C D C l}_{3}\right): \delta 7.86(\mathrm{~d}, J=1.9 \mathrm{~Hz}, 1 \mathrm{H}), 7.63(\mathrm{dd}, J=8.4,1.9 \mathrm{~Hz}, 1 \mathrm{H}), 7.35(\mathrm{~m}, 5 \mathrm{H}), 6.38(\mathrm{~m}, 2 \mathrm{H}), 6.25$ (d, $J=6.4 \mathrm{~Hz}, 1 \mathrm{H}), 5.50$ (dd, $J=10.2,1.1 \mathrm{~Hz}, 1 \mathrm{H}), 5.42(\mathrm{dd}, J=16.9,1.0 \mathrm{~Hz}, 1 \mathrm{H})$.

${ }^{13}$ C NMR (75 MHz, $\left.\mathbf{C D C l}_{3}\right): \delta 181.9,157.2,149.1,145.9,135.8,133.8,131.5,129.1,128.4,127.2,120.8,119.6,115.4$, 86.3, 57.1.

FT-IR (thin film): 3065, 3033, 2985, 2921, 2254, 1954, 1891, 1733, 1603, 1494, 1464, 1431, 1370, 1320, 1285, 1188, 1129, 1078, 1054, 1030, 993, 909, 820, 775, 734, 700, $643 \mathrm{~cm}^{-1}$.

HRMS: Calculated for $\mathrm{C}_{17} \mathrm{H}_{12} \mathrm{INO}_{2}[\mathrm{M}+\mathrm{H}]^{+} 389.9991$, found 389.9985 .

HPLC: 99:1 er (Daicel CHIRALPAK AS-H, 90:10 heptane $/ \mathrm{i} \mathrm{PrOH}, 1.0 \mathrm{~mL} / \mathrm{min} ; T_{\text {major }}=9.3 \mathrm{~min}, T_{\text {minor }}=17.4 \mathrm{~min}$ ) $[\alpha]_{\mathbf{D}}{ }^{24}:+45.1^{\circ}\left(c=2.6, \mathrm{CHCl}_{3}\right)$
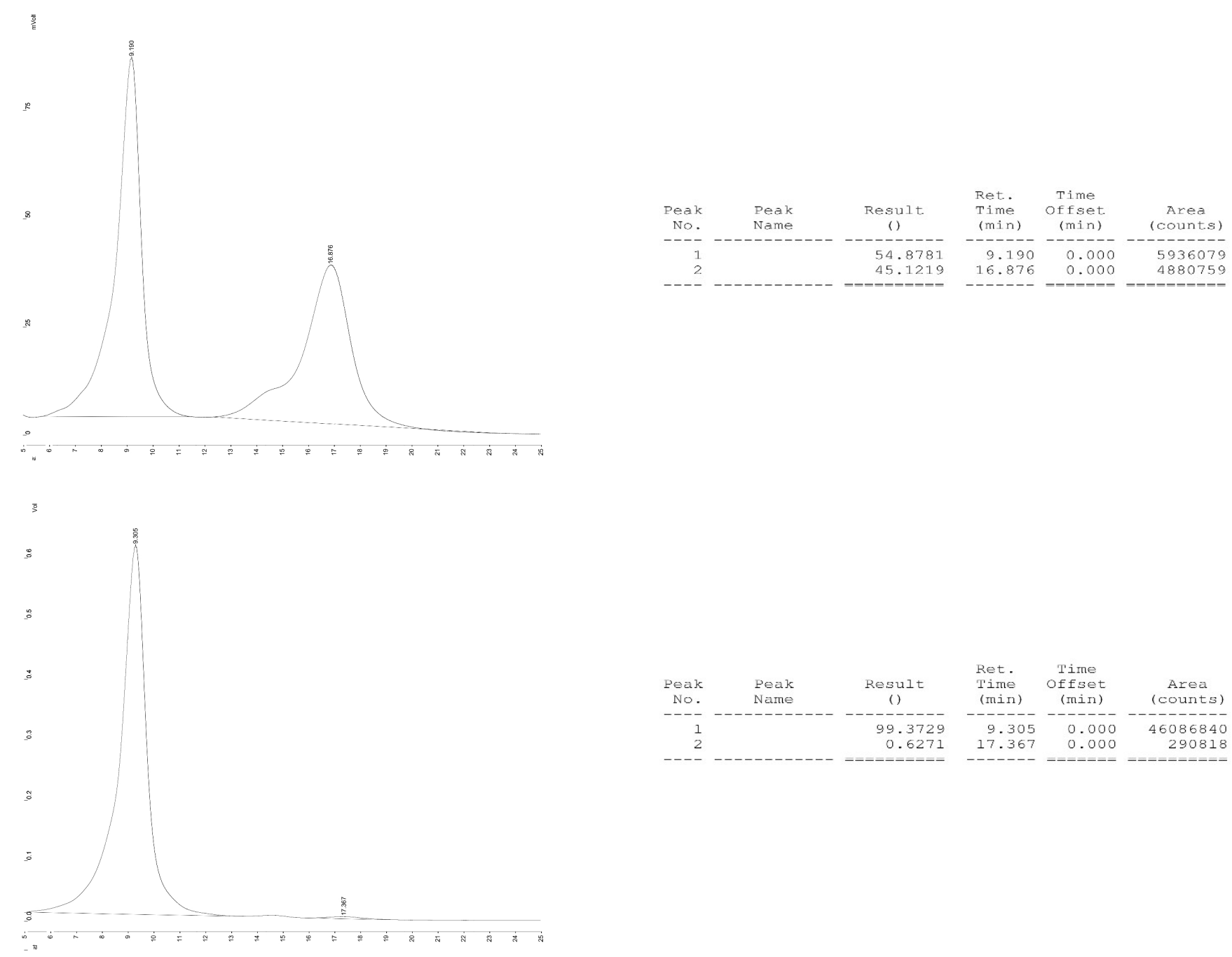


\section{1-[(R)-1-Phenyl-2-propenyl]-6-chloro-2,3-indolinedione (9fa)}

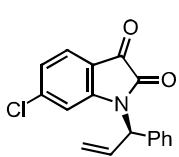

The compound was prepared according to general procedure A using 6-chloroisatin $7 \mathbf{f}(90.8 \mathrm{mg}, 0.50 \mathrm{mmol}$, 1.00 equiv) and cinnamyl chloride 8a (70 $\mu \mathrm{L}, 0.50 \mathrm{mmol}, 1.00$ equiv). Crude ${ }^{1} \mathrm{H}$ NMR showed $>20: 1$ branched/linear selectivity. Purification by flash chromatography (silica, 5:1 petroleum ether/EtOAc) afforded 9fa as a bright yellow solid. (119 mg, 80\%)

Physical appearance: Bright yellow solid

Melting point: Changes appearance at $79^{\circ} \mathrm{C}$; completely melts by $104^{\circ} \mathrm{C}$

TLC $\mathbf{R}_{\mathbf{f}}$ (5:1 petroleum ether/EtOAc): 0.53

${ }^{1}$ H NMR (300 MHz, CDCl $)$ ): $\delta 7.55(\mathrm{~d}, J=8.0 \mathrm{~Hz}, 1 \mathrm{H}), 7.38(\mathrm{~m}, 5 \mathrm{H}), 7.04(\mathrm{dd}, J=8.0,1.7 \mathrm{~Hz}, 1 \mathrm{H}), 6.62(\mathrm{~s}, 1 \mathrm{H}), 6.41$ (ddd, $J=16.9,10.3,6.4 \mathrm{~Hz}, 1 \mathrm{H}), 6.24(\mathrm{~d}, J=6.3 \mathrm{~Hz}, 1 \mathrm{H}), 5.53$ (d, $J=10.3 \mathrm{~Hz}, 1 \mathrm{H}), 5.43(\mathrm{~d}, J=17.0 \mathrm{~Hz}, 1 \mathrm{H})$.

${ }^{13}$ C NMR (101 MHz, CDCl $): \delta 181.8$ (C), 158.2 (C), 150.9 (C), 144.3 (C), 135.9 (C), 131.7 (CH), $129.3(\mathrm{CH}), 128.6$ $(\mathrm{CH}), 127.3(\mathrm{CH}), 126.5(\mathrm{CH}), 124.1(\mathrm{CH}), 120.9\left(\mathrm{CH}_{2}\right), 116.4(\mathrm{C}), 113.8(\mathrm{CH}), 57.4(\mathrm{CH})$.

FT-IR (thin film): 3088, 3032, 2921, 1896, 1744, 1609, 1494, 1476, 1433, 1343, 1290, 1261, 1200, 1181, 1105, 1075, 992, 945, 907, 835, 792, 742, $699 \mathrm{~cm}^{-1}$.

HRMS: Calculated for $\mathrm{C}_{17} \mathrm{H}_{12} \mathrm{ClNO}_{2}[\mathrm{M}+\mathrm{H}]^{+} 298.0635$, found 298.0628 .

HPLC: $91: 9 \mathrm{er}$ (Daicel CHIRALPAK IA, 97:3 heptane $/{ }^{2} \mathrm{PrOH}, 0.8 \mathrm{~mL} / \mathrm{min} ; T_{\text {major }}=22.4 \mathrm{~min}, T_{\text {minor }}=14.7 \mathrm{~min}$ ) $[\alpha]_{\mathbf{D}}{ }^{24}:+12.4^{\circ}\left(c=0.90, \mathrm{CHCl}_{3}\right)$
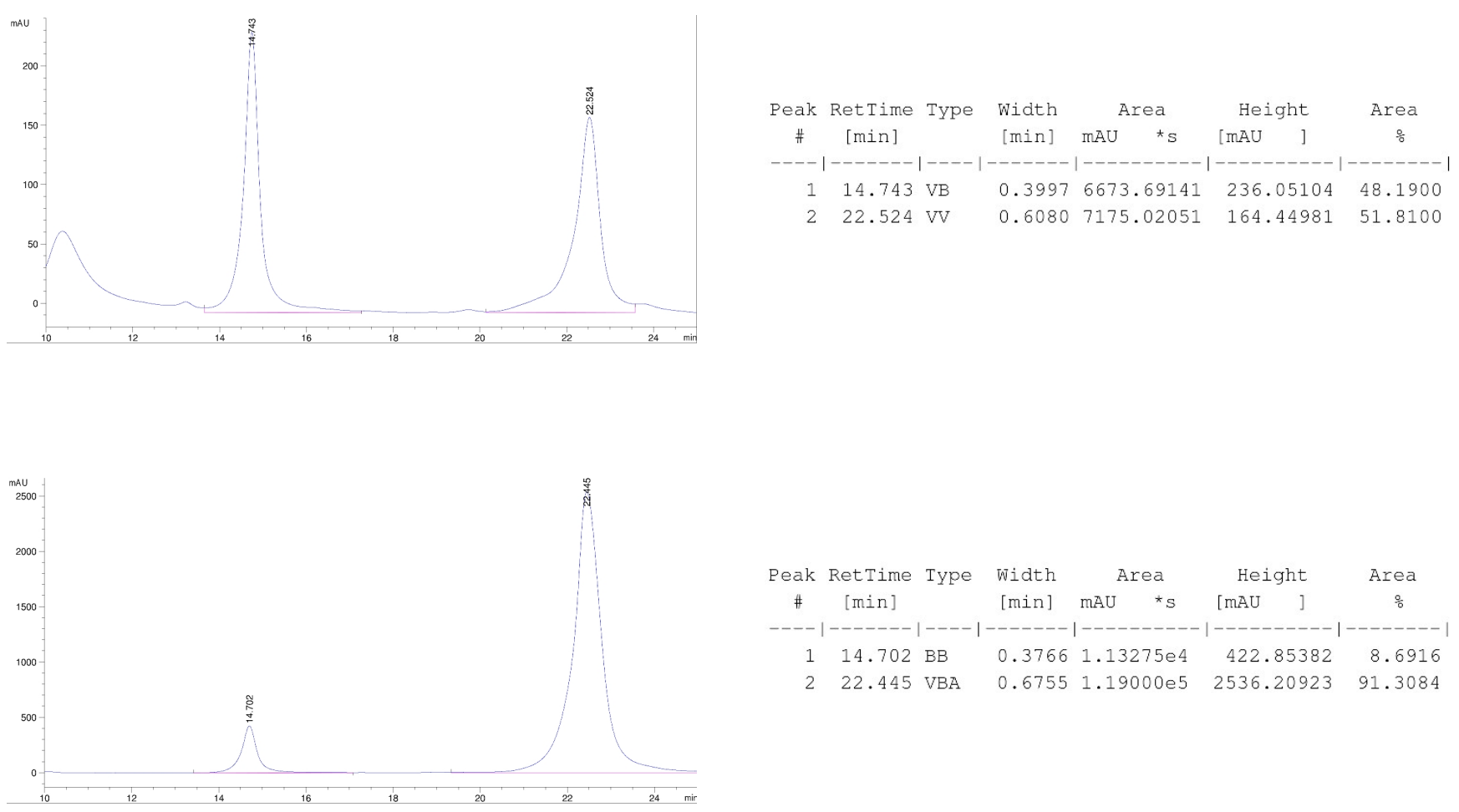


\section{1-[(R)-1-Phenyl-2-propenyl]-6-bromo-2,3-indolinedione (9ga)}

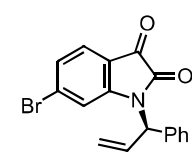

The compound was prepared according to general procedure A using 6-bromoisatin $7 \mathrm{~g}(113.0 \mathrm{mg}, 0.50 \mathrm{mmol}$, 1.00 equiv) and cinnamyl chloride 8a (70 $\mu \mathrm{L}, 0.50 \mathrm{mmol}, 1.00$ equiv). Crude ${ }^{1} \mathrm{H}$ NMR showed $>20: 1$ branched/linear selectivity. Purification by flash chromatography (silica, 10:1 to 7:1 petroleum ether/EtOAc) afforded 9ga as a bright orange solid. (123 $\mathrm{mg}, 72 \%)$

Physical appearance: Bright orange solid

Melting point: Changes appearance at $80{ }^{\circ} \mathrm{C}$; completely melts by $103{ }^{\circ} \mathrm{C}$

TLC R $\mathbf{R}_{\mathbf{f}}$ (7:1 petroleum ether/EtOAc): 0.43

${ }^{1} \mathbf{H}$ NMR (300 MHz, $\left.\mathbf{C D C l}_{3}\right): \delta 7.46(\mathrm{~d}, J=8.0 \mathrm{~Hz}, 1 \mathrm{H}), 7.38(\mathrm{~m}, 5 \mathrm{H}), 7.21(\mathrm{dd}, J=8.0,1.4 \mathrm{~Hz}, 1 \mathrm{H}), 6.80(\mathrm{~d}, J=1.4 \mathrm{~Hz}$, $1 \mathrm{H}), 6.41$ (ddd, $J=16.9,10.3,6.5 \mathrm{~Hz}, 1 \mathrm{H}), 6.22$ (d, $J=6.5 \mathrm{~Hz}, 1 \mathrm{H}), 5.52$ (dt, $J=10.3,1.1 \mathrm{~Hz}, 1 \mathrm{H}), 5.43(\mathrm{ddd}, J=17.0$, $1.6,0.9 \mathrm{~Hz}, 1 \mathrm{H})$.

${ }^{13}$ C NMR (75 MHz, $\left.\mathbf{C D C l}_{3}\right): \delta 182.0,158.0,150.6,135.8,133.2,131.6,129.2,128.5,127.2,127.0,126.4,120.9,116.7$, $116.5,57.3$.

FT-IR (thin film): 3088, 3064, 3031, 2986, 2921, 1887, 1745, 1607, 1495, 1471, 1451, 1429, 1343, 1261, 1200, 1181, 1106, 1062, 991, 944, 903, 836, 791, 736, $700 \mathrm{~cm}^{-1}$.

HRMS: Calculated for $\mathrm{C}_{17} \mathrm{H}_{12} \mathrm{BrNO}_{2}[\mathrm{M}+\mathrm{H}]^{+} 342.0130$, found 342.0124.

HPLC: 94.5:5.5 er (Daicel CHIRALPAK IA, 97:3 heptane $/{ }^{i} \mathrm{PrOH}, 0.8 \mathrm{~mL} / \mathrm{min} ; T_{\text {major }}=19.6 \mathrm{~min}, T_{\operatorname{minor}}=14.3 \mathrm{~min}$ ) $[\alpha]_{\mathbf{D}}^{24}:+14.6^{\circ}\left(c=1.9, \mathrm{CHCl}_{3}\right)$
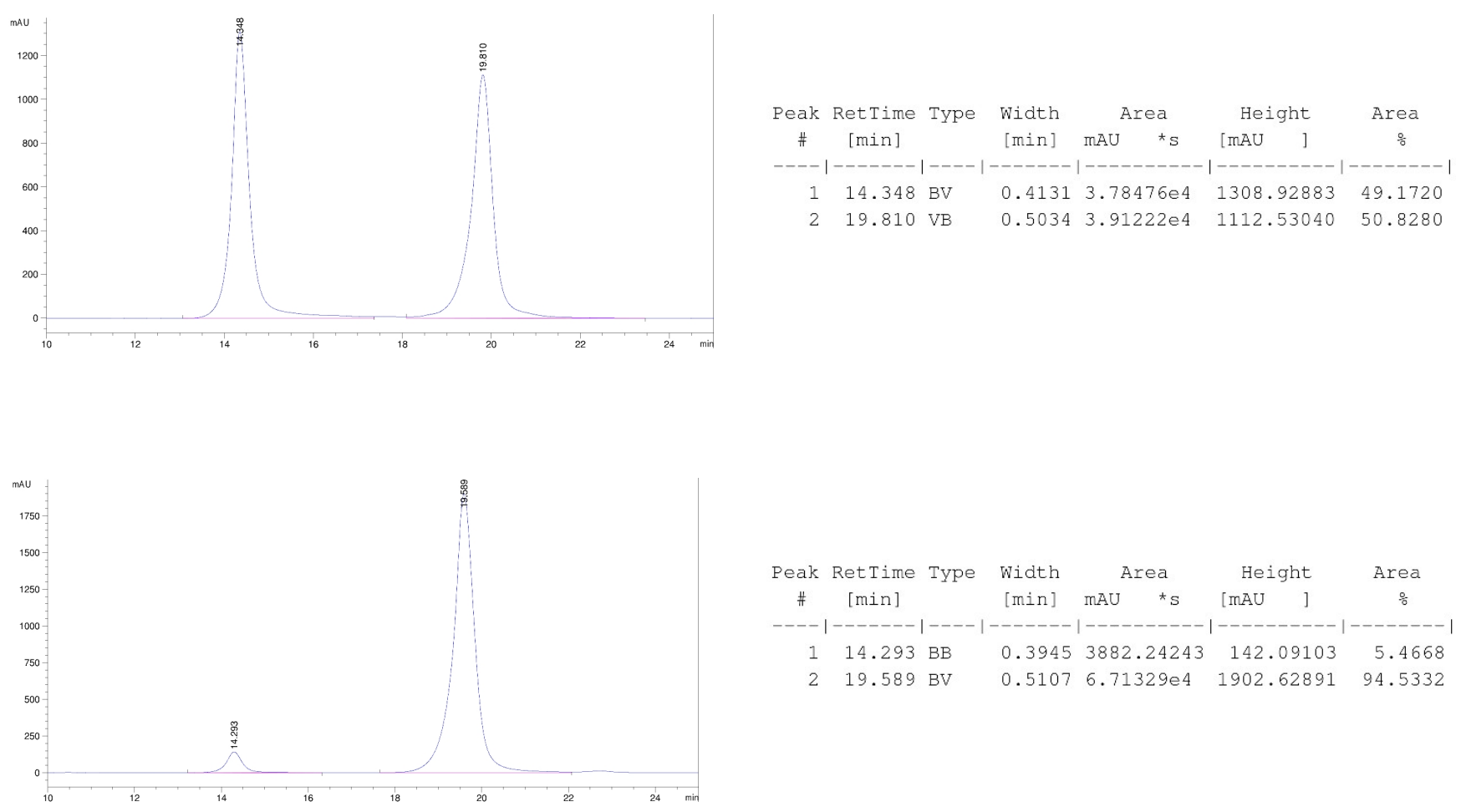


\section{1-[(R)-1-Phenyl-2-propenyl]-7-bromo-2,3-indolinedione (9ha)}

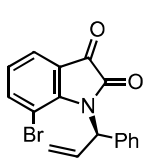

The title compound was prepared according to general procedure B using 7-bromoisatin $\mathbf{7 h}(113.0 \mathrm{mg}, 0.500$ mmol, 1.00 equiv) and cinnamyl chloride 8a (70 $\mu \mathrm{L}, 0.50 \mathrm{mmol}, 1.00$ equiv). Crude ${ }^{1} \mathrm{H}$ NMR showed 3:1 branched/linear selectivity. Purification by flash chromatography (silica, 7:1 petroleum ether/EtOAc) afforded 9ha as a bright orange solid. (97 mg, 55\%)

Physical appearance: Bright orange solid

Melting point: $113-119^{\circ} \mathrm{C}$

TLC $R_{\mathbf{f}}$ (7:1 petroleum ether/EtOAc): 0.30

${ }^{1} \mathbf{H}$ NMR (400 MHz, CDCl $)$ : $\delta 7.72(\mathrm{~d}, J=8.1 \mathrm{~Hz}, 1 \mathrm{H}), 7.63(\mathrm{~d}, J=7.3 \mathrm{~Hz}, 1 \mathrm{H}), 7.34(\mathrm{~m}, 5 \mathrm{H}), 7.01(\mathrm{dd}, J=8.3,7.3 \mathrm{~Hz}$, $1 \mathrm{H}), 6.90(\mathrm{~d}, J=7.4 \mathrm{~Hz}, 1 \mathrm{H}), 6.71$ (ddd, $J=17.2,9.8,7.5 \mathrm{~Hz}, 1 \mathrm{H}), 5.44(\mathrm{~m}, 2 \mathrm{H})$.

${ }^{13}$ C NMR (101 MHz, CDCl $): \delta 182.1(\mathrm{C}), 158.8(\mathrm{C}), 148.1(\mathrm{C}), 144.7(\mathrm{CH}), 138.2(\mathrm{C}), 134.2(\mathrm{CH}), 128.7(\mathrm{CH}), 127.8$ $(\mathrm{CH}), 126.8(\mathrm{br}, \mathrm{CH}), 125.2(\mathrm{CH}), 125.1(\mathrm{CH}), 121.6(\mathrm{C}), 120.2$ (br, $\left.\mathrm{CH}_{2}\right), 104.4$ (br, C), 59.5 (br, $\left.\mathrm{CH}\right)$.

FT-IR (thin film): 3084, 3030, 2983, 2923, 1744, 1608, 1572, 1494, 1468, 1420, 1340, 1283, 1240, 1158, 1118, 1072 , 1026, 1001, 941, 842, 824, 769, $699 \mathrm{~cm}^{-1}$.

HRMS: Calculated for $\mathrm{C}_{17} \mathrm{H}_{12} \mathrm{BrNO}_{2}[\mathrm{M}+\mathrm{H}]^{+} 342.0130$, found 342.0121 .

HPLC: 76.5:23.5 er (Daicel CHIRALPAK OJ-H, 80:20 heptane $/{ }^{i} \mathrm{PrOH}, 1.0 \mathrm{~mL} / \mathrm{min} ; T_{\text {major }}=24.1 \mathrm{~min}, T_{\text {minor }}=20.2 \mathrm{~min}$ ) $[\alpha]_{\mathbf{D}}{ }^{24}:+3.6^{\circ}\left(c=0.30, \mathrm{CHCl}_{3}\right)$
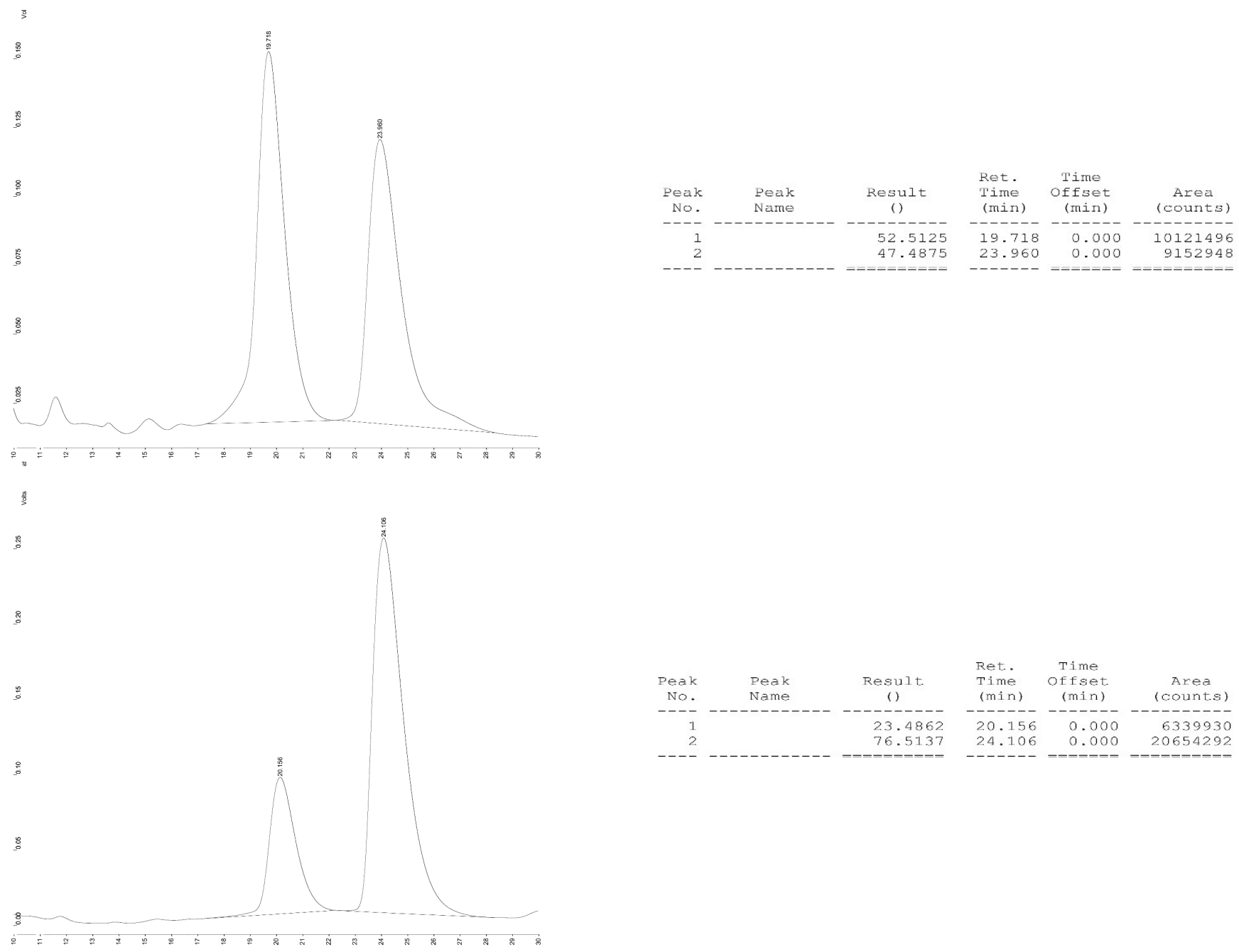


\section{1-[(R)-1-Phenyl-2-propenyl]-4,6-difluoro-2,3-indolinedione (9ia)}

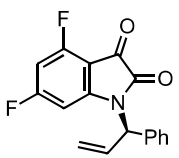

The title compound was prepared according to general procedure B using 4,6-difluoroisatin 7i $(91.6 \mathrm{mg}, 0.500$ mmol, 1.00 equiv) and cinnamyl chloride $8 \mathbf{a}\left(70 \mu \mathrm{L}, 0.50 \mathrm{mmol}, 1.00\right.$ equiv). Crude ${ }^{1} \mathrm{H}$ NMR showed 13:1 branched/linear selectivity. Purification by flash chromatography (7 $\mathrm{g}$ silica, 7:1 petroleum ether/EtOAc) afforded 9ia as a bright yellow-green solid. (60 $\mathrm{mg}, 40 \%)$

Physical appearance: Bright yellow-green solid

Melting point: Changes appearance at $49^{\circ} \mathrm{C}$; completely melts by $110^{\circ} \mathrm{C}$

TLC $\mathbf{R}_{\mathbf{f}}$ (7:1 petroleum ether/EtOAc): 0.42

${ }^{1}$ H NMR (400 MHz, CDCl $\left.)_{3}\right): \delta 7.37(\mathrm{~m}, 5 \mathrm{H}), 6.40(\mathrm{~m}, 2 \mathrm{H}), 6.24(\mathrm{~d}, J=6.6 \mathrm{~Hz}, 1 \mathrm{H}), 6.16(\mathrm{~m}, 1 \mathrm{H}), 5.54(\mathrm{dd}, J=10.3,1.2$ $\mathrm{Hz}, 1 \mathrm{H}), 5.43(\mathrm{dd}, J=17.1,1.4 \mathrm{~Hz}, 1 \mathrm{H})$.

${ }^{13}$ C NMR (101 MHz, CDCl $): ~ \delta 177.0,169.5(\mathrm{dd}, J=261.9,13.2 \mathrm{~Hz}), 160.3(\mathrm{dd}, J=269.2,15.5 \mathrm{~Hz}), 157.9,151.8,135.6$, 131.4, 129.3, 128.7, 127.2, 121.1, 113.7, 99.8 (dd, $J=26.7,23.2 \mathrm{~Hz}), 98.5(\mathrm{dd}, J=28.4,3.6 \mathrm{~Hz}), 57.7$.

${ }^{19}$ F NMR (376 MHz, $\left.\mathbf{C D C l}_{3}\right): \delta-89.27(\mathrm{dt}, J=17.7,9.2 \mathrm{~Hz}),-101.88(\mathrm{dd}, J=16.8,8.6 \mathrm{~Hz})$.

FT-IR (thin film): 3085, 3033, 2920, 1746, 1629, 1495, 1448, 1392, 1345, 1267, 1207, 1144, 1084, 998, 950, 841, 797, $753,711,626 \mathrm{~cm}^{-1}$.

HRMS: Calculated for $\mathrm{C}_{17} \mathrm{H}_{11} \mathrm{~F}_{2} \mathrm{NO}_{2}[\mathrm{M}+\mathrm{H}]^{+} 300.0836$, found 300.0829 .

HPLC: 99:1 er (Daicel CHIRALPAK IA, 97:3 heptane $/ / \mathrm{PrOH}, 0.8 \mathrm{~mL} / \mathrm{min} ; T_{\text {major }}=13.6 \mathrm{~min}, T_{\text {minor }}=12.0 \mathrm{~min}$ )

$[\alpha]_{\mathbf{D}}{ }^{24}:+52.0^{\circ}\left(c=0.93, \mathrm{CHCl}_{3}\right)$
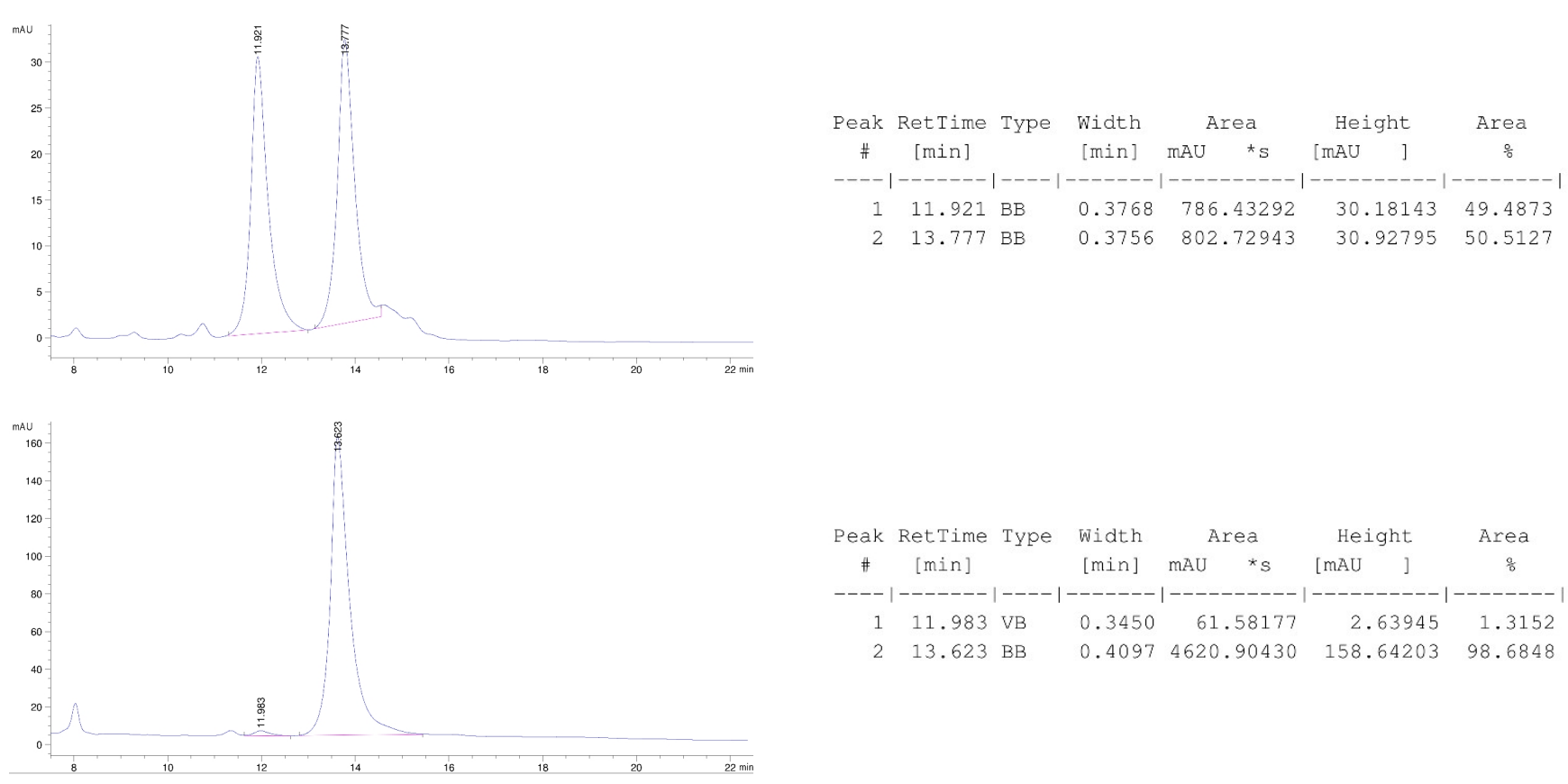


\section{1-[(R)-1-Phenyl-2-propenyl]-5,6-dichloro-2,3-indolinedione (9ja)}

(1)

The title compound was prepared according to general procedure B using 5,6-dichloroisatin $7 \mathbf{j}$ (108 mg, 0.500 mmol, 1.00 equiv) and cinnamyl chloride 8a (70 $\mu \mathrm{L}, 0.50 \mathrm{mmol}, 1.00$ equiv). Crude ${ }^{1} \mathrm{H}$ NMR showed 4:1 branched/linear selectivity. Purification by flash chromatography $(9 \mathrm{~g}$ silica, 8:1 $\rightarrow$ 4:1 petroleum ether/EtOAc) afforded $\mathbf{9 j a}$ as an orange solid. (98 $\mathrm{mg}, 59 \%)$

Physical appearance: Orange solid

Melting point: Changes appearance at $105^{\circ} \mathrm{C}$; completely melts by $120^{\circ} \mathrm{C}$

TLC $\mathbf{R}_{\mathbf{f}}$ (5:1 petroleum ether/EtOAc, UV): 0.31

${ }^{1}$ H NMR (400 MHz, $\left.\mathbf{C D C l}_{3}\right): \delta 7.67(\mathrm{~s}, 1 \mathrm{H}), 7.37(\mathrm{~m}, 5 \mathrm{H}), 6.72(\mathrm{~s}, 1 \mathrm{H}), 6.39(\mathrm{ddd}, J=16.9,10.3,6.4 \mathrm{~Hz}, 1 \mathrm{H}), 6.25(\mathrm{~d}, J=$ $6.5 \mathrm{~Hz}, 1 \mathrm{H}), 5.54(\mathrm{~d}, J=10.4 \mathrm{~Hz}, 1 \mathrm{H}), 5.43(\mathrm{~d}, J=17.1 \mathrm{~Hz}, 1 \mathrm{H})$.

${ }^{13}$ C NMR (101 MHz, CDCl $): \delta 181.1(\mathrm{C}), 157.6(\mathrm{C}), 148.4(\mathrm{C}), 142.0(\mathrm{C}), 135.5$ (C), $131.4(\mathrm{CH}), 129.3(\mathrm{CH}), 128.7$ $(\mathrm{CH}), 128.2(\mathrm{C}), 127.2(\mathrm{CH}), 126.7(\mathrm{CH}), 121.0\left(\mathrm{CH}_{2}\right), 117.3(\mathrm{C}), 115.3(\mathrm{CH}), 57.4(\mathrm{CH})$.

FT-IR (thin film): 3062, 2920, 1744, 1612, 1577, 1493, 1458, 1410, 1365, 1324, 1268, 1232, 1171, 1109, 1004, 937, 853, $783,751,701 \mathrm{~cm}^{-1}$.

HRMS: Calculated for $\mathrm{C}_{17} \mathrm{H}_{11} \mathrm{Cl}_{2} \mathrm{NO}_{2}[\mathrm{M}+\mathrm{H}]^{+} 332.0245$, found 332.0233 .

HPLC: 84:16 er (Daicel CHIRALPAK AD-H, 90:10 heptane $/ \mathrm{PrOH}, 1.0 \mathrm{~mL} / \mathrm{min} ; T_{\text {major }}=14.8 \mathrm{~min}, T_{\text {minor }}=10.4 \mathrm{~min}$ ) $[\alpha]_{\mathbf{D}}{ }^{25}:+31.0^{\circ}\left(c=1.0, \mathrm{CHCl}_{3}\right)$
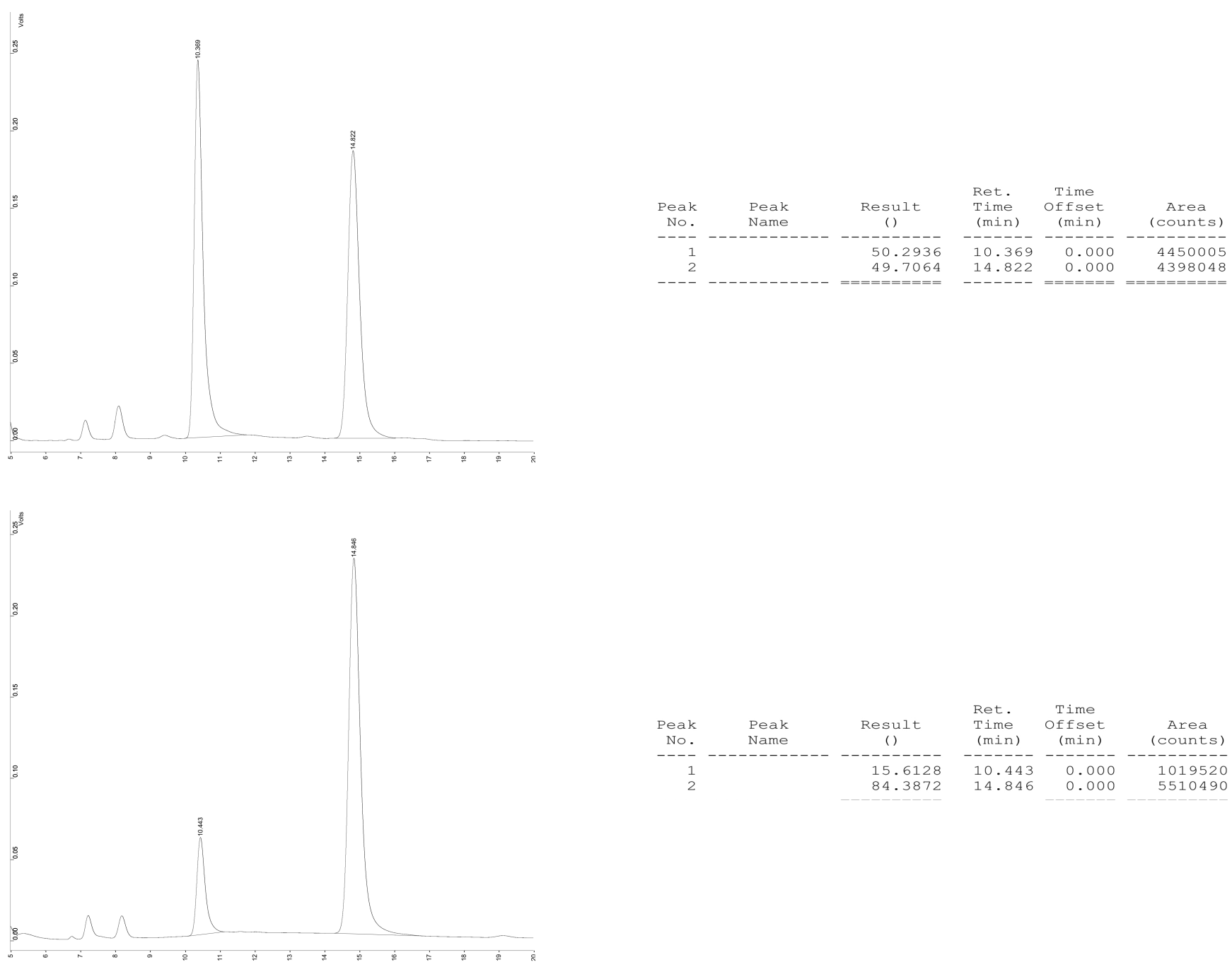


\section{1-[(R)-1-Phenyl-2-propenyl]-5-methoxy-2,3-indolinedione (9ka)}

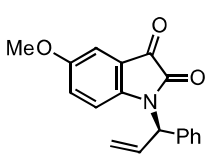

The title compound was prepared according to general procedure B using 5-methoxyisatin $7 \mathbf{k}(89 \mathrm{mg}, 0.50$ mmol, 1.0 equiv) and cinnamyl chloride 8a (70 $\mu \mathrm{L}, 0.50 \mathrm{mmol}, 1.00$ equiv). Crude ${ }^{1} \mathrm{H}$ NMR showed 7:1 branched/linear selectivity. Purification by flash chromatography (10 g silica, 6:1 petroleum ether/EtOAc $\rightarrow$ EtOAc) afforded $9 \mathrm{ka}$ as a dark red oil. (89 mg, 61\%)

Physical appearance: Dark red oil

TLC $R_{\mathbf{f}}$ (3:1 petroleum ether/EtOAc): 0.24

${ }^{1}$ H NMR (300 MHz, $\left.\mathbf{C D C l}_{3}\right): \delta 7.58(\mathrm{~d}, J=8.5 \mathrm{~Hz}, 1 \mathrm{H}), 7.37(\mathrm{~s}, 5 \mathrm{H}), 6.49(\mathrm{dd}, J=8.5,2.1 \mathrm{~Hz}, 1 \mathrm{H}), 6.40$ (ddd, $J=16.9$, $10.4,6.5 \mathrm{~Hz}, 1 \mathrm{H}), 6.23(\mathrm{~d}, J=6.3 \mathrm{~Hz}, 1 \mathrm{H}), 6.08(\mathrm{~m}, 1 \mathrm{H}), 5.49(\mathrm{~d}, J=10.3 \mathrm{~Hz}, 1 \mathrm{H}), 5.42(\mathrm{~d}, J=17.0 \mathrm{~Hz}, 1 \mathrm{H}), 3.72(\mathrm{~s}, 3 \mathrm{H})$.

${ }^{13}$ C NMR (10 MHz, CDCl $)$ ): $\delta 183.7$ (C), $158.3(\mathrm{C}), 156.3(\mathrm{C}), 143.7(\mathrm{C}), 136.4(\mathrm{C}), 132.1(\mathrm{CH}), 129.0(\mathrm{CH}), 128.2(\mathrm{CH})$, $127.3(\mathrm{CH}), 124.4(\mathrm{CH}), 120.5\left(\mathrm{CH}_{2}\right), 118.6(\mathrm{C}), 114.3(\mathrm{CH}), 109.5(\mathrm{CH}), 57.0(\mathrm{CH}), 56.0\left(\mathrm{CH}_{3}\right)$.

FT-IR (thin film): 3065, 3030, 2980, 2944, 2846, 1729, 1616, 1498, 1452, 1367, 1293, 1225, 1171, 1112, 1027, 993, 936, $842,790,762,726,702,644 \mathrm{~cm}^{-1}$.

HRMS: Calculated for $\mathrm{C}_{18} \mathrm{H}_{15} \mathrm{NO}_{3}[\mathrm{M}+\mathrm{H}]^{+} 294.1130$, found 294.1124.

HPLC: 89:11 er (Daicel CHIRALPAK AS, 80:20 heptane $/ \mathrm{PrOH}, 1.0 \mathrm{~mL} / \mathrm{min} ; T_{\text {major }}=8.4 \mathrm{~min}, T_{\text {minor }}=16.7 \mathrm{~min}$ )

$[\alpha]_{\mathrm{D}}{ }^{24}:+115.6^{\circ}\left(c=0.28, \mathrm{CHCl}_{3}\right)$; at higher concentrations, $[\alpha]_{\mathrm{D}}$ values for this compound were highly erratic
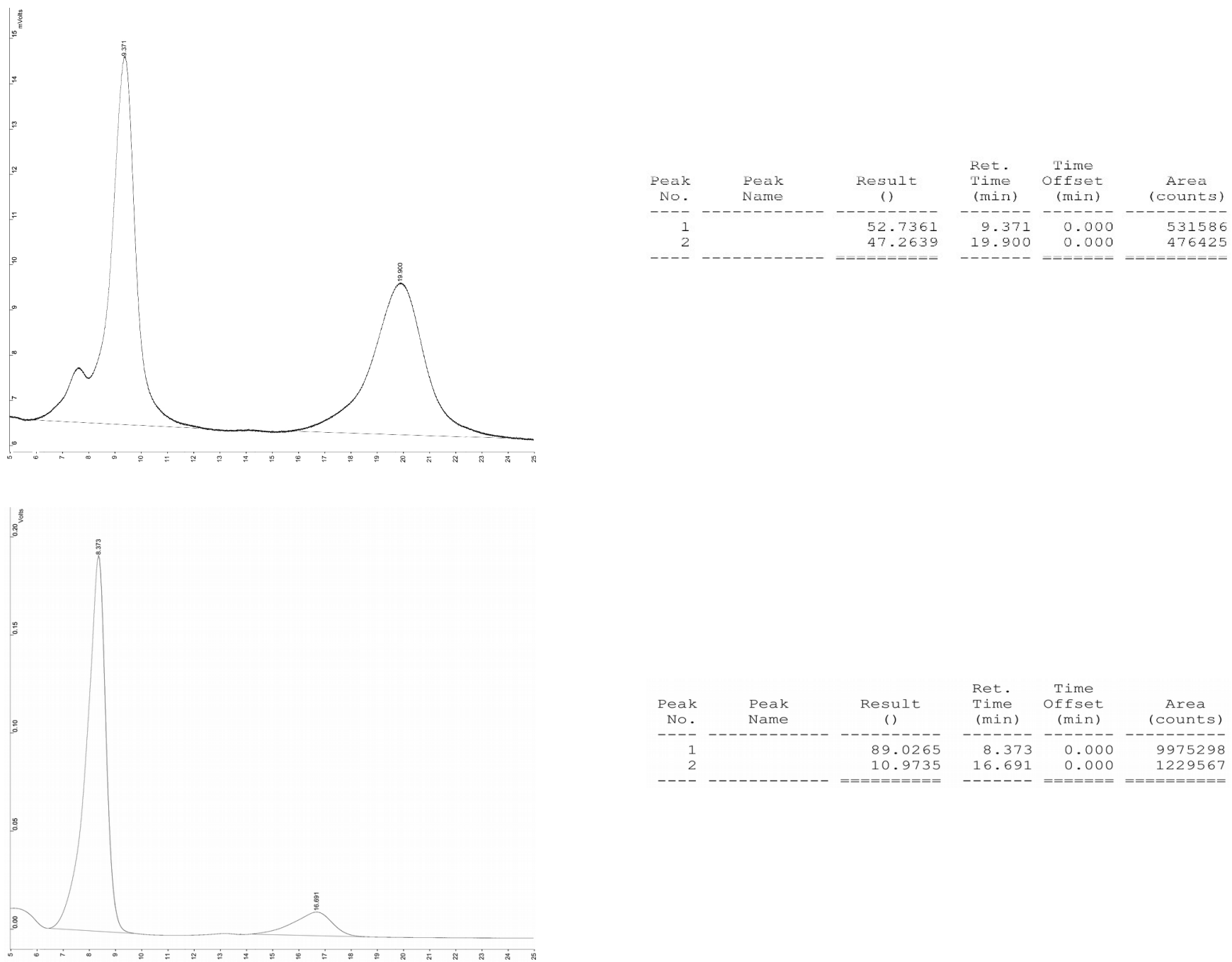


\section{1-[(R)-1-Phenyl-2-propenyl]-6-methoxy-2,3-indolinedione (9la)}

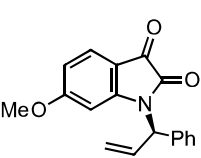

The title compound was prepared according to general procedure B using 6-methoxyisatin 71 ( $89 \mathrm{mg}, 0.50$ mmol, 1.0 equiv) and cinnamyl chloride $8 \mathrm{a}\left(70 \mu \mathrm{L}, 0.50 \mathrm{mmol}, 1.0\right.$ equiv). Crude ${ }^{1} \mathrm{H}$ NMR showed 8:1 branched/linear selectivity. Purification by flash chromatography (silica, 5:1 petroleum ether/EtOAc $\rightarrow$ EtOAc) afforded 9la as a yellow solid. (111 mg, 75\%)

Physical appearance: Yellow solid

Melting point: Changes appearance at $81^{\circ} \mathrm{C}$; completely melts by $123{ }^{\circ} \mathrm{C}$

TLC $\mathbf{R}_{\mathbf{f}}$ (7:1 petroleum ether/EtOAc): 0.23

${ }^{1}$ H NMR (300 MHz, CDCl $): \delta 7.29(\mathrm{~m}, 5 \mathrm{H}), 7.07(\mathrm{~d}, J=2.8 \mathrm{~Hz}, 1 \mathrm{H}), 6.84(\mathrm{dd}, J=8.7,2.8 \mathrm{~Hz}, 1 \mathrm{H}), 6.43(\mathrm{dd}, J=8.7,0.5$ $\mathrm{Hz}, 1 \mathrm{H}), 6.32$ (ddd, $J=16.9,10.3,6.6 \mathrm{~Hz}, 1 \mathrm{H}), 6.16$ (d, $J=6.6 \mathrm{~Hz}, 1 \mathrm{H}), 5.39(\mathrm{~m}, 2 \mathrm{H}), 3.68(\mathrm{~s}, 3 \mathrm{H})$.

${ }^{13}$ C NMR (101 MHz, CDCl $): \delta 180.5$ (C), 167.7 (C), 159.6 (C), 152.3 (C), 136.5 (C), $132.1(\mathrm{CH}), 129.0(\mathrm{CH}), 128.2$ $(\mathrm{CH}), 127.9(\mathrm{CH}), 127.3(\mathrm{CH}), 120.3\left(\mathrm{CH}_{2}\right), 111.7(\mathrm{C}), 108.1(\mathrm{CH}), 100.3(\mathrm{CH}), 57.0(\mathrm{CH}), 56.0\left(\mathrm{CH}_{3}\right)$.

FT-IR (thin film): 3062, 3030, 2934, 2838, 1734, 1624, 1597, 1489, 1454, 1368, 1326, 1278, 1252, 1185, 1153, 1006, 935, $824,765,724,700 \mathrm{~cm}^{-1}$.

HRMS: Calculated for $\mathrm{C}_{18} \mathrm{H}_{15} \mathrm{NO}_{3}[\mathrm{M}+\mathrm{H}]^{+} 294.1130$, found 294.1125.

HPLC: 87:13 er (Daicel CHIRALPAK AD-H, 90:10 heptane $/ \mathrm{PrOH}, 1.0 \mathrm{~mL} / \mathrm{min} ; T_{\text {major }}=30.5 \mathrm{~min}, T_{\text {minor }}=23.3 \mathrm{~min}$ ) $[\alpha]_{\mathbf{D}}{ }^{25}:+55.5^{\circ}\left(c=1.0, \mathrm{CHCl}_{3}\right)$
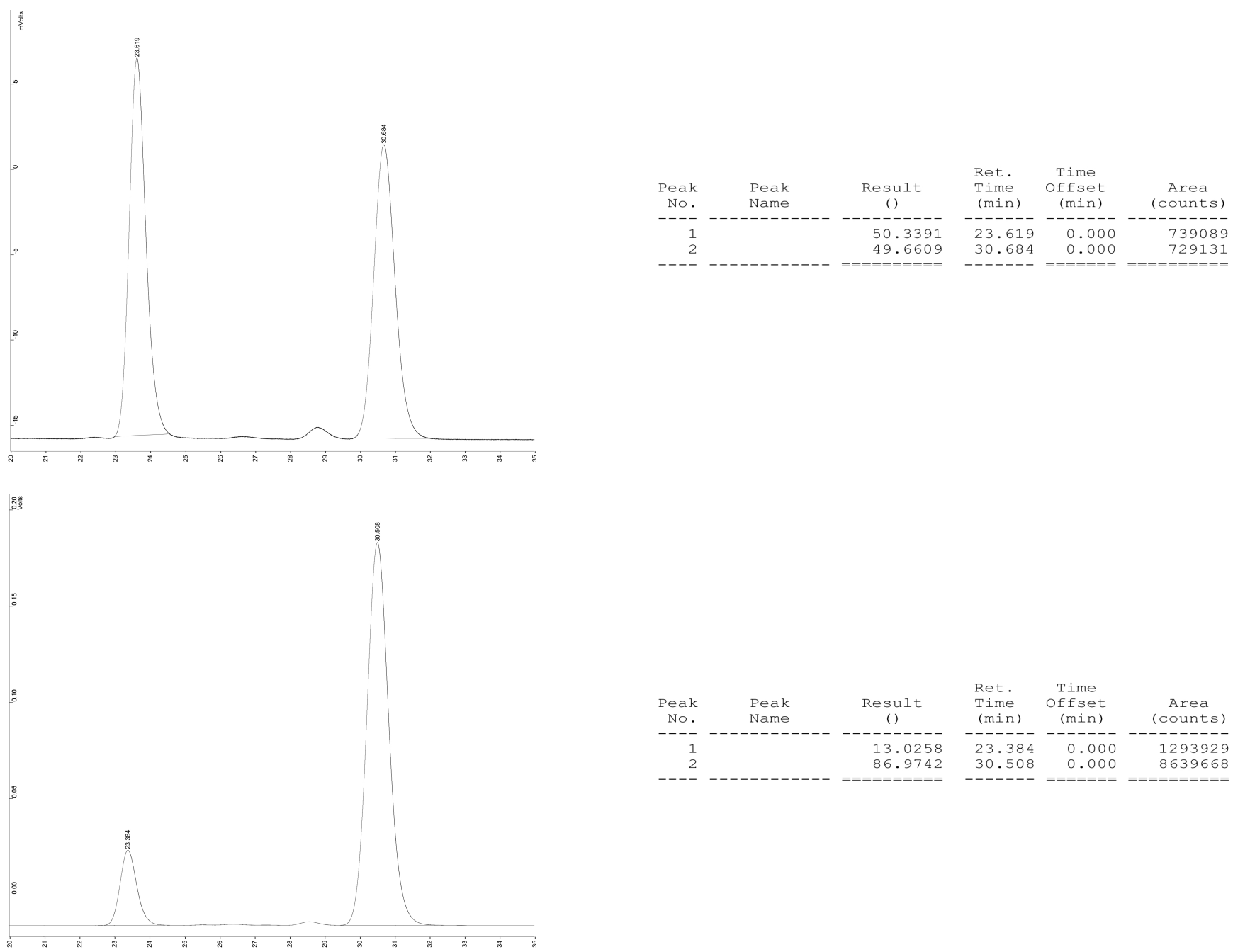


\section{1-[(R)-1-Phenyl-2-propenyl]-5-methyl-2,3-indolinedione (9ma)}

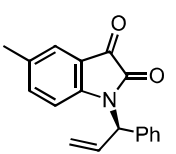

The title compound was prepared according to general procedure B using 5-methylisatin $7 \mathbf{m}(80.6 \mathrm{mg}, 0.500$ mmol, 1.00 equiv) and cinnamyl chloride $8 \mathbf{a}\left(70 \mu \mathrm{L}, 0.50 \mathrm{mmol}, 1.00\right.$ equiv). Crude ${ }^{1} \mathrm{H}$ NMR showed $>20: 1$ branched/linear selectivity. Purification by flash chromatography (silica, 10:1 to 7:1 petroleum ether/EtOAc) afforded $9 \mathrm{ma}$ as a bright red solid. (97 $\mathrm{mg}, 70 \%)$

Physical appearance: Bright red solid

Melting point: Changes appearance at $58^{\circ} \mathrm{C}$; completely melts by $117^{\circ} \mathrm{C}$

TLC $\mathbf{R}_{\mathbf{f}}$ (7:1 petroleum ether/EtOAc): 0.36

${ }^{1} \mathbf{H}$ NMR $\left(300 \mathrm{MHz}, \mathbf{C D C l}_{3}\right): \delta 7.37(\mathrm{~m}, 6 \mathrm{H}), 7.17(\mathrm{~m}, 1 \mathrm{H}), 6.50(\mathrm{~d}, J=8.1 \mathrm{~Hz}, 1 \mathrm{H}), 6.41(\mathrm{ddd}, J=17.0,10.3,6.6 \mathrm{~Hz}, 1 \mathrm{H})$, $6.23(\mathrm{~d}, J=6.6 \mathrm{~Hz}, 1 \mathrm{H}), 5.46(\mathrm{~m}, 2 \mathrm{H}), 2.27(\mathrm{~s}, 3 \mathrm{H})$

${ }^{13}$ C NMR (101 MHz, $\left.\mathbf{C D C l}_{3}\right): \delta 183.6,158.3,147.6,138.5,136.4,133.5,132.0,129.0,128.2,127.2,125.8,120.5,118.1$, $113.1,57.0,20.7$.

FT-IR (thin film): 3062, 3031, 2923, 2864, 1734, 1622, 1595, 1489, 1450, 1369, 1328, 1185, 1131, 1078, 991, 935, 818, $765,723,701,646 \mathrm{~cm}^{-1}$.

HRMS: Calculated for $\mathrm{C}_{18} \mathrm{H}_{15} \mathrm{NO}_{2}[\mathrm{M}+\mathrm{H}]^{+} 278.1181$, found 278.1176 .

HPLC: $91: 9 \mathrm{er}$ (Daicel CHIRALPAK IA, 97:3 heptane $/{ }^{i} \mathrm{PrOH}, 0.8 \mathrm{~mL} / \mathrm{min} ; T_{\text {major }}=15.5 \mathrm{~min}, T_{\text {minor }}=14.4 \mathrm{~min}$ ) $[\alpha]_{\mathbf{D}}{ }^{24}:+75.0^{\circ}\left(c=2.1, \mathrm{CHCl}_{3}\right)$
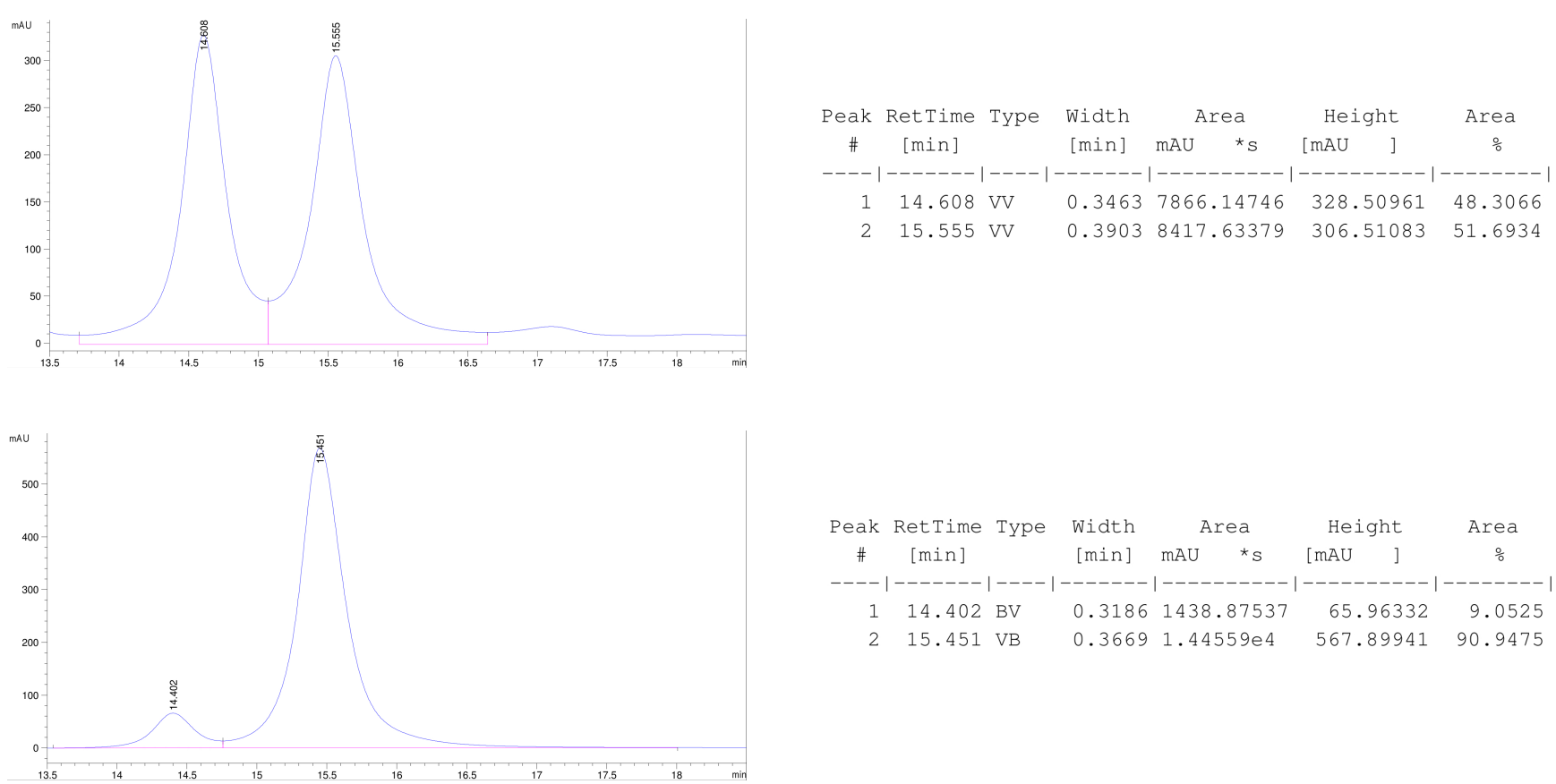

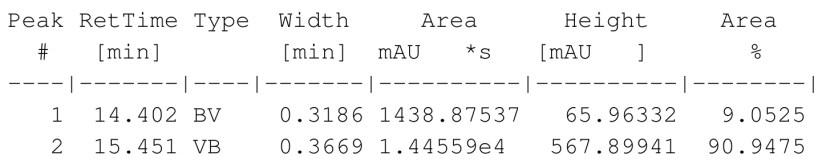




\section{1-[(R)-1-Phenyl-2-propenyl]-4,6-dimethyl-2,3-indolinedione (9na)}

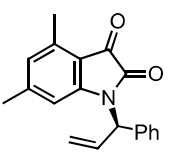

The title compound was prepared according to general procedure B using 4,6-dimethylisatin $7 \mathbf{n}(87.6 \mathrm{mg}$, $0.500 \mathrm{mmol}, 1.00$ equiv) and cinnamyl chloride $8 \mathbf{a}\left(70 \mu \mathrm{L}, 0.50 \mathrm{mmol}, 1.00\right.$ equiv). Crude ${ }^{1} \mathrm{H}$ NMR showed 4:1 branched/linear selectivity. Purification by flash chromatography (6 g silica, 5:1 $\rightarrow$ 2:1 petroleum ether/EtOAc) afforded 9na as an orange solid. (90 mg, 62\%)

Physical appearance: Orange solid

Melting point: $111-115^{\circ} \mathrm{C}$

TLC $\mathbf{R}_{\mathbf{f}}$ (5:1 petroleum ether/EtOAc, UV): 0.26

${ }^{1}$ H NMR (400 MHz, CDCl $\left.)_{3}\right): \delta 7.34(\mathrm{~m}, 5 \mathrm{H}), 6.63(\mathrm{~s}, 1 \mathrm{H}), 6.43(\mathrm{ddd}, J=17.1,10.3,6.7 \mathrm{~Hz}, 1 \mathrm{H}), 6.25(\mathrm{~s}, 1 \mathrm{H}), 6.20(\mathrm{~d}, J=$ $6.8 \mathrm{~Hz}, 1 \mathrm{H}), 5.47(\mathrm{~d}, J=10.3 \mathrm{~Hz}, 1 \mathrm{H}), 5.42(\mathrm{~d}, J=17.1 \mathrm{~Hz}, 1 \mathrm{H}), 2.51(\mathrm{~s}, 3 \mathrm{H}), 2.20(\mathrm{~s}, 3 \mathrm{H})$.

${ }^{13}$ C NMR (101 MHz, CDCl $): \delta 182.9(\mathrm{C}), 158.8(\mathrm{C}), 150.2(\mathrm{C}), 149.3(\mathrm{C}), 141.2(\mathrm{C}), 136.7(\mathrm{C}), 132.3(\mathrm{CH}), 128.9(\mathrm{CH})$, 128.0 (CH), $127.1(\mathrm{CH}), 126.6(\mathrm{CH}), 120.4\left(\mathrm{CH}_{2}\right), 114.1(\mathrm{C}), 111.3(\mathrm{CH}), 57.0(\mathrm{CH}), 22.9\left(\mathrm{CH}_{3}\right), 18.2\left(\mathrm{CH}_{3}\right)$.

FT-IR (thin film): 3029, 2973, 2919, 2852, 1723, 1610, 1492, 1451, 1414, 1371, 1340, 1267, 121, 1157, 1088, 1029, 989, 947, 908, 839, 794, 749, $701 \mathrm{~cm}^{-1}$.

HRMS: Calculated for $\mathrm{C}_{19} \mathrm{H}_{17} \mathrm{NO}_{2}[\mathrm{M}+\mathrm{H}]^{+} 292.1338$, found 292.1339 .

HPLC: 99.5:0.5 er (Daicel CHIRALPAK AD-H, 90:10 heptane $/ \mathrm{i} \mathrm{PrOH}, 1.0 \mathrm{~mL} / \mathrm{min} ; T_{\text {major }}=9.5 \mathrm{~min}, T_{\text {minor }}=8.6 \mathrm{~min}$ ) $[\alpha]_{\mathbf{D}}{ }^{25}:+40.8^{\circ}\left(c=1.0, \mathrm{CHCl}_{3}\right)$
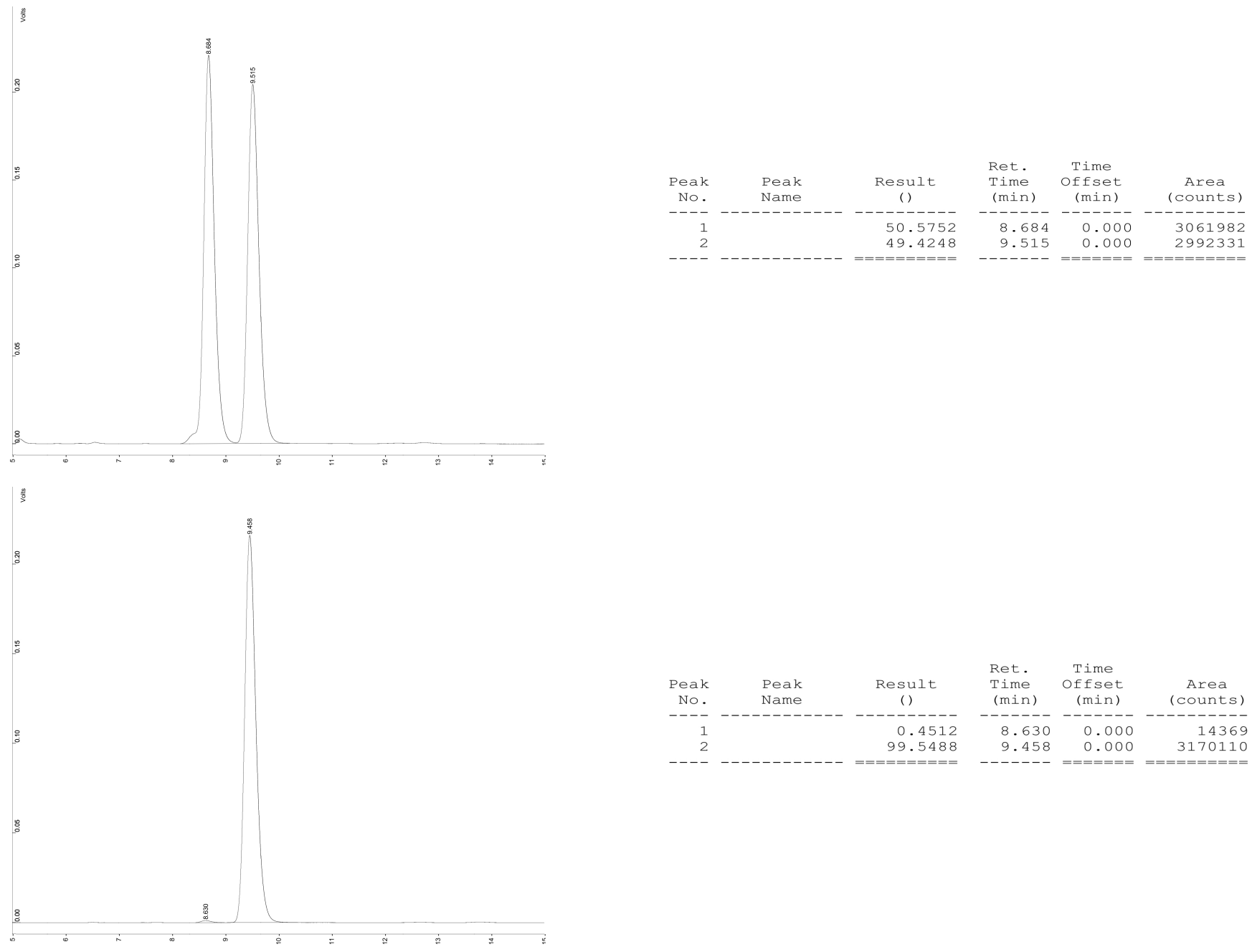


\section{5-[(R)-1-Phenyl-2-propenyl]-5-azatricyclo[7.4.0.0 $\left.{ }^{2,6}\right]$ trideca-1(9),2(6),7,10,12-pentaene-3,4-dione (9oa)}

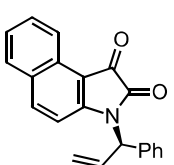

The title compound was prepared according to general procedure B using 5-azatricyclo[7.4.0.0. $\left.0^{2,6}\right]$ trideca1(9),2(6),7,10,12-pentaene-3,4-dione $7 \mathbf{o}$ (98.6 mg, $0.500 \mathrm{mmol}, 1.00$ equiv) and cinnamyl chloride $8 \mathbf{a}$ (70 $\mu \mathrm{L}$, $0.50 \mathrm{mmol}, 1.00$ equiv). Crude ${ }^{1} \mathrm{H}$ NMR showed 17:1 branched/linear selectivity. Purification by flash chromatography ( $8 \mathrm{~g}$ silica, $5: 1 \rightarrow 1: 1$ petroleum ether/EtOAc) afforded $90 a$ as a dark red solid. (100 $\mathrm{mg}, 64 \%$ )

Physical appearance: Dark red solid

Melting point: $127-132{ }^{\circ} \mathrm{C}$

TLC $\mathbf{R}_{\mathbf{f}}$ (3:1 petroleum ether/EtOAc, $\left.\mathbf{U V}\right): 0.34$

${ }^{1}$ H NMR (400 MHz, CDCl $): \delta 8.64(\mathrm{~d}, J=8.4 \mathrm{~Hz}, 1 \mathrm{H}), 7.85(\mathrm{~d}, J=8.8 \mathrm{~Hz}, 1 \mathrm{H}), 7.68(\mathrm{~d}, J=8.3 \mathrm{~Hz}, 1 \mathrm{H}), 7.59(\mathrm{t}, J=7.6 \mathrm{~Hz}$, 1H), $7.36(\mathrm{~m}, 6 \mathrm{H}), 6.83(\mathrm{~d}, J=8.8 \mathrm{~Hz}, 1 \mathrm{H}), 6.46$ (ddd, $J=17.0,10.3,6.5 \mathrm{~Hz}, 1 \mathrm{H}), 6.30(\mathrm{~d}, J=6.6 \mathrm{~Hz}, 1 \mathrm{H}), 5.49(\mathrm{~m}, 2 \mathrm{H})$.

${ }^{13}$ C NMR (101 MHz, CDCl $): \delta 182.4(\mathrm{C}), 158.8(\mathrm{C}), 153.7(\mathrm{C}), 139.9(\mathrm{CH}), 136.6(\mathrm{C}), 132.3(\mathrm{CH}), 131.2(\mathrm{CH}), 129.9$

(C), $129.2(\mathrm{C}), 129.1(\mathrm{CH}), 128.9(\mathrm{CH}), 128.2(\mathrm{CH}), 127.1(\mathrm{CH}), 125.7(\mathrm{CH}), 123.3(\mathrm{CH}), 120.5\left(\mathrm{CH}_{2}\right), 113.1(\mathrm{CH}), 109.7$ (C), $56.8(\mathrm{CH})$.

FT-IR (thin film): 3062, 2920, 1743, 1716, 1628, 1573, 1529, 1495, 1455, 1406, 1378, 1321, 1225, 1145, 1096, 992, 938 , $880,825,753,718,700 \mathrm{~cm}^{-1}$.

HRMS: Calculated for $\mathrm{C}_{21} \mathrm{H}_{15} \mathrm{NO}_{2}[\mathrm{M}+\mathrm{H}]^{+} 314.1181$, found 314.1180.

HPLC: 94:6 er (Daicel CHIRALPAK AD-H, 90:10 heptane $/ 2 \mathrm{PrOH}, 1.0 \mathrm{~mL} / \mathrm{min} ; T_{\text {major }}=18.4 \mathrm{~min}, T_{\text {minor }}=32.8 \mathrm{~min}$ ) $[\alpha]_{\mathbf{D}}{ }^{25}:+232.5^{\circ}\left(c=0.50, \mathrm{CHCl}_{3}\right)$
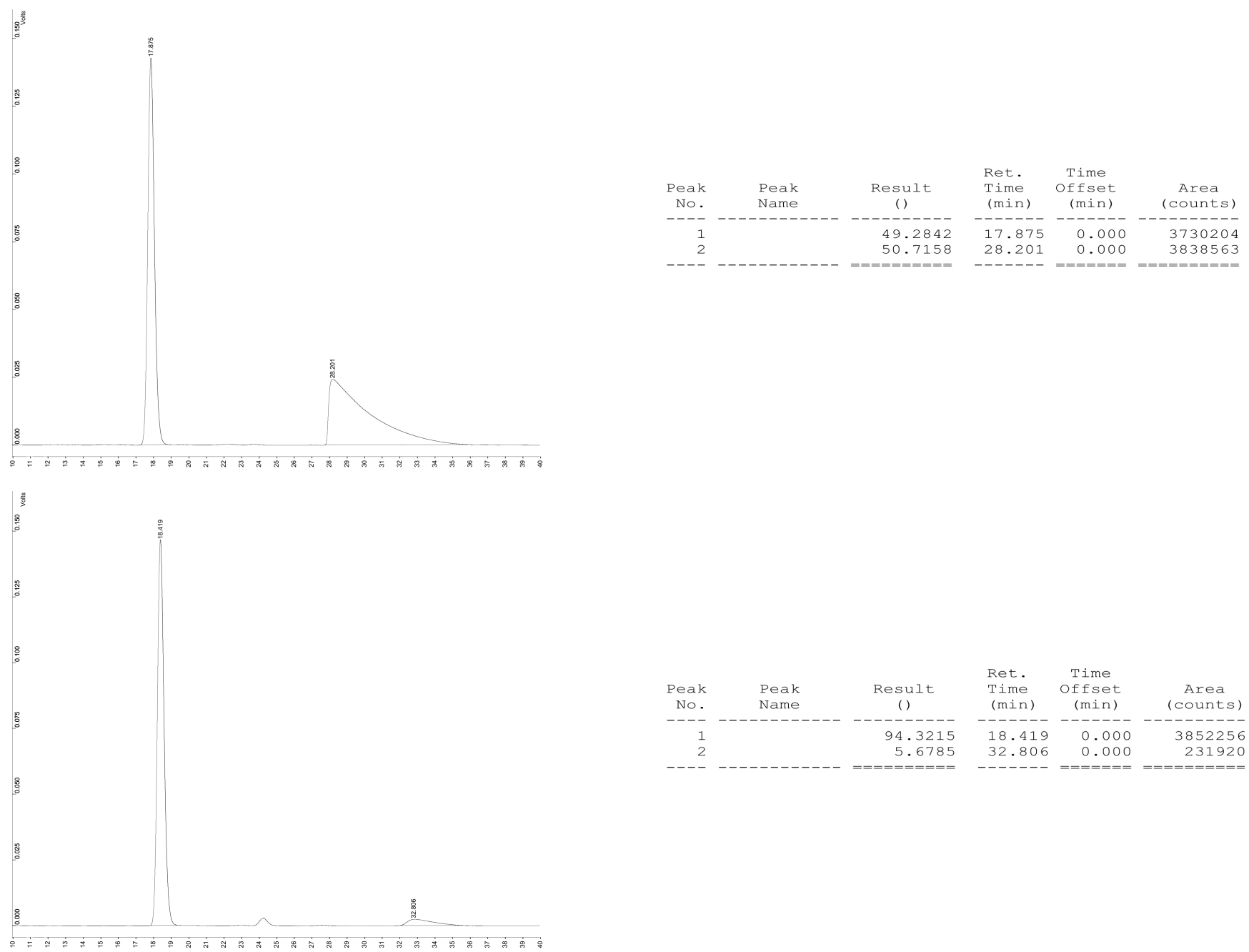


\section{1-[(R)-1-(o-Tolyl)-2-propenyl]-2,3-indolinedione (9ab)}

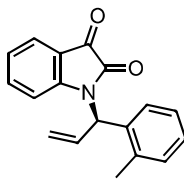

The title compound was prepared according to general procedure B using isatin 7a $(73.6 \mathrm{mg}, 0.500 \mathrm{mmol}$, 1.00 equiv) and cinnamyl chloride $\mathbf{8 b}(83 \mathrm{mg}, 0.50 \mathrm{mmol}, 1.00$ equiv) with the following modification: the reaction vial was charged with $\mathbf{7 a}, \mathbf{8 b}$, and solvent $(50 \mu \mathrm{L})$, followed by the catalyst solution and DIPEA. After 18 hours, $26 \%$ conversion of cinnamyl chloride $8 \mathbf{b}$ was observed by ${ }^{1} \mathrm{H}$ NMR, so a solution of $\mathrm{CpRu}(\mathrm{MeCN})_{3} \mathrm{PF}_{6}(1.1 \mathrm{mg}, 0.0025 \mathrm{mmol}, 0.0050$ equiv) and ligand $\mathbf{L 1}(2.9 \mathrm{mg}, 0.0030 \mathrm{mmol}, 0.0060$ equiv) in dimethyl carbonate $(50 \mu \mathrm{L})$ was added and the reaction was stirred at $40{ }^{\circ} \mathrm{C}$ overnight. At this point, crude ${ }^{1} \mathrm{H}$ NMR showed $90 \%$ conversion of cinnamyl chloride $\mathbf{8 b}$ 18:1 branched/linear selectivity. Purification by flash chromatography (12 g silica, 5:1 $\rightarrow$ 1:1 petroleum ether/EtOAc) afforded 9 ab as a bright orange solid. (86 $\mathrm{mg}, 62 \%)$

Physical appearance: Bright orange solid

Melting point: Changes appearance at $60^{\circ} \mathrm{C}$; completely melts by $117^{\circ} \mathrm{C}$

TLC $R_{\mathbf{f}}$ (3:1 petroleum ether/EtOAc): 0.29

${ }^{1} \mathbf{H}$ NMR (300 MHz, $\left.\mathbf{C D C l}_{3}\right)$ : $\delta 7.59$ (dd, $\left.J=7.5,1.4 \mathrm{~Hz}, 1 \mathrm{H}\right), 7.52(\mathrm{dd}, J=5.3,3.8 \mathrm{~Hz}, 1 \mathrm{H}), 7.33(\mathrm{~m}, 1 \mathrm{H}), 7.26$ (ddd, $J=$ $5.3,3.8,1.3 \mathrm{~Hz}, 2 \mathrm{H}), 7.19(\mathrm{dd}, J=5.3,3.8 \mathrm{~Hz}, 1 \mathrm{H}), 7.03(\mathrm{t}, J=7.5 \mathrm{~Hz}, 1 \mathrm{H}), 6.70(\mathrm{dd}, J=8.0,0.8 \mathrm{~Hz}, 1 \mathrm{H}), 6.38$ (ddd, $J=$ $17.2,10.4,5.4 \mathrm{~Hz}, 1 \mathrm{H}), 6.23(\mathrm{~d}, J=5.4 \mathrm{~Hz}, 1 \mathrm{H}), 5.45(\mathrm{ddd}, J=10.3,1.8,0.8 \mathrm{~Hz}, 1 \mathrm{H}), 5.29$ (ddd, $J=17.1,1.8,0.8 \mathrm{~Hz}, 1 \mathrm{H})$, $2.26(\mathrm{~s}, 3 \mathrm{H})$.

${ }^{13}$ C NMR (101 MHz, $\left.\mathbf{C D C l}_{3}\right): \delta 183.2(\mathrm{C}), 157.8(\mathrm{C}), 150.7$ (C), $138.1(\mathrm{CH}), 137.4(\mathrm{C}), 134.4(\mathrm{C}), 132.8(\mathrm{CH}), 131.4$ $(\mathrm{CH}), 128.7(\mathrm{CH}), 128.2(\mathrm{CH}), 126.3(\mathrm{CH}), 125.4(\mathrm{CH}), 123.6(\mathrm{CH}), 118.6\left(\mathrm{CH}_{2}\right), 118.0(\mathrm{C}), 113.0(\mathrm{CH}), 55.6(\mathrm{CH}), 19.7$ $\left(\mathrm{CH}_{3}\right)$.

FT-IR (thin film): 3067, 3025, 2924, 1739, 1611, 1466, 1412, 1371, 1345, 1300, 1195, 1159, 1094, 991, 934, $818 \mathrm{~cm}^{-1}$.

HRMS: Calculated for $\mathrm{C}_{18} \mathrm{H}_{15} \mathrm{NO}_{2}[\mathrm{M}+\mathrm{H}]^{+} 278.1181$, found 278.1174 .

HPLC: 83:17 er (Daicel CHIRALPAK OJ-H, 90:10 heptane $/ 2 \mathrm{PrOH}, 1.0 \mathrm{~mL} / \mathrm{min} ; T_{\text {major }}=34.3 \mathrm{~min}, T_{\text {minor }}=26.3 \mathrm{~min}$ ) $[\alpha]_{\mathbf{D}}{ }^{25}:+94.3^{\circ}\left(c=1.0, \mathrm{CHCl}_{3}\right)$
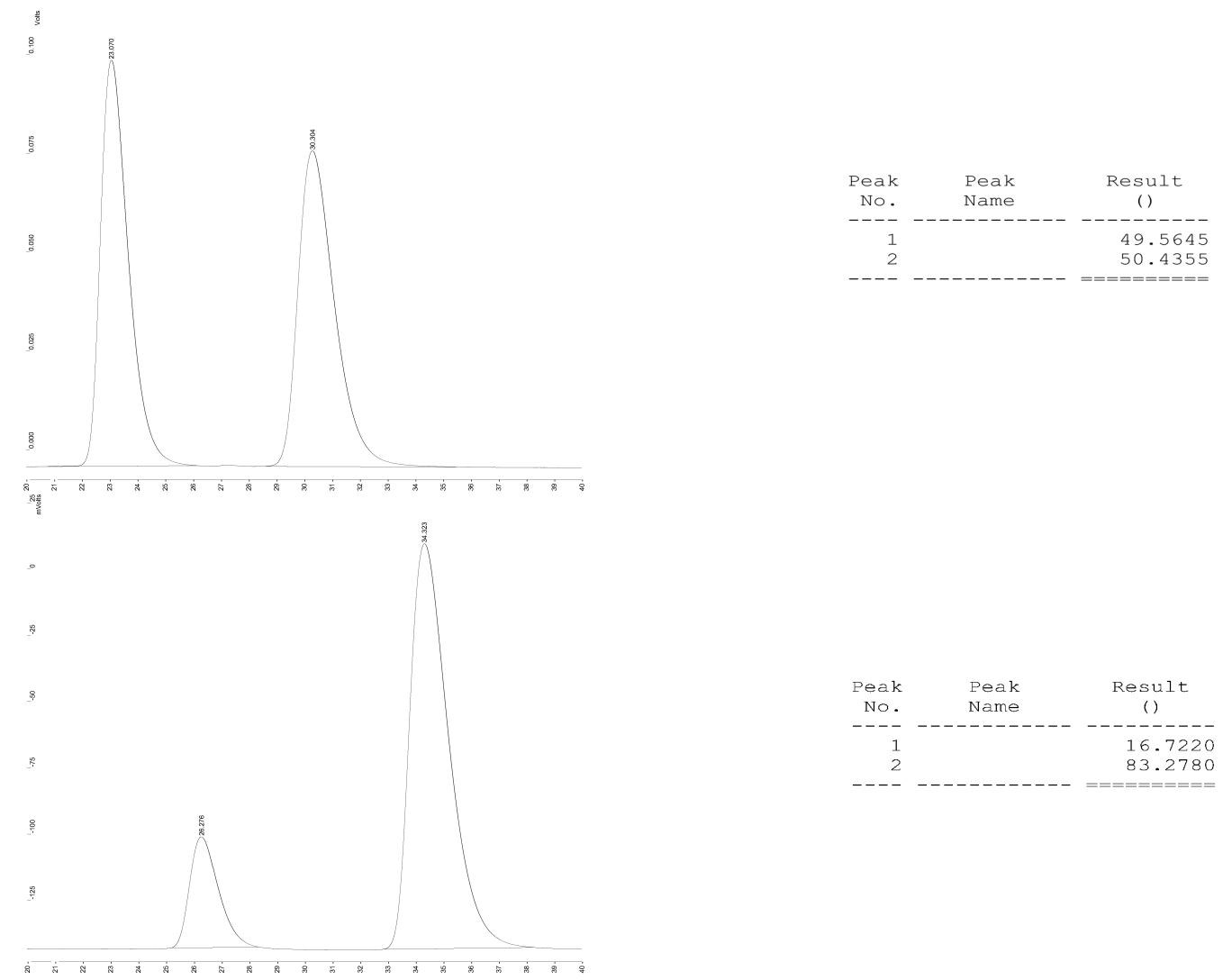

\begin{tabular}{lrr}
$\begin{array}{l}\text { Ret. } \\
\text { Time }\end{array}$ & $\begin{array}{c}\text { Time } \\
\text { Offset } \\
\text { (min) }\end{array}$ & $\begin{array}{c}\text { Area } \\
\text { (counts) }\end{array}$ \\
----- & ----- & -------- \\
23.070 & 0.000 & 7153200 \\
30.304 & 0.000 & 7278911 \\
\hline
\end{tabular}

\begin{tabular}{|c|c|c|c|c|c|}
\hline $\begin{array}{l}\text { Peak } \\
\text { No. }\end{array}$ & $\begin{array}{l}\text { Peak } \\
\text { Name }\end{array}$ & $\begin{array}{c}\text { Result } \\
\text { () }\end{array}$ & $\begin{array}{l}\text { Ret. } \\
\text { Time } \\
\text { (min) }\end{array}$ & $\begin{array}{l}\text { Time } \\
\text { Offset } \\
\text { (min) }\end{array}$ & $\begin{array}{c}\text { Area } \\
\text { (counts) }\end{array}$ \\
\hline 1 & & 16.7220 & 26.276 & 0.000 & 2975399 \\
\hline 2 & & 83.2780 & 34.323 & 0.000 & 14817885 \\
\hline
\end{tabular}




\section{1-[(R)-1-(m-Methoxyphenyl)-2-propenyl]-2,3-indolinedione (9ac)}

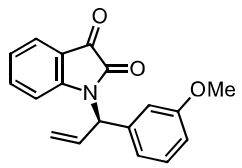

The title compound was prepared according to general procedure A using isatin $7 \mathbf{a}(73.6 \mathrm{mg}, 0.500 \mathrm{mmol}$, 1.00 equiv) and cinnamyl chloride $8 \mathbf{c}(91.3 \mathrm{mg}, 0.50 \mathrm{mmol}, 1.00$ equiv) with the following modification: the reaction vial was charged with $7 \mathbf{a}, \mathbf{8 c}$, and solvent $(50 \mu \mathrm{L})$, followed by the catalyst solution and DIPEA. After 18 hours, $67 \%$ conversion of cinnamyl chloride 8c was observed by ${ }^{1} \mathrm{H}$ NMR, so a solution of $\mathrm{CpRu}(\mathrm{MeCN})_{3} \mathrm{PF}_{6}(1.1 \mathrm{mg}, 0.0025 \mathrm{mmol}, 0.0050$ equiv) and ligand $\mathbf{L 1}$ (2.9 $\mathrm{mg}, 0.0030 \mathrm{mmol}, 0.0060$ equiv) in 3pentanone $(50 \mu \mathrm{L})$ was added and the reaction was stirred at $40{ }^{\circ} \mathrm{C}$ overnight. At this point, crude ${ }^{1} \mathrm{H}$ NMR showed full conversion of cinnamyl chloride $\mathbf{8 c}$ and 15:1 branched/linear selectivity. Purification by flash chromatography (13 $\mathrm{g}$ silica, 4:1 petroleum ether/EtOAc $\rightarrow$ EtOAc) afforded 9ac as an orange oil. (77 mg, 52\%)

Physical appearance: Orange oil

TLC $\mathbf{R}_{\mathbf{f}}$ (3:1 petroleum ether/EtOAc): 0.24

${ }^{1} \mathrm{H}$ NMR (400 MHz, $\left.\mathbf{C D C l}_{3}\right): \delta 7.61(\mathrm{~d}, J=7.5 \mathrm{~Hz}, 1 \mathrm{H}), 7.37(\mathrm{td}, J=7.8,1.3 \mathrm{~Hz}, 1 \mathrm{H}), 7.29(\mathrm{t}, J=7.6 \mathrm{~Hz}, 1 \mathrm{H}), 7.05(\mathrm{td}, J$ $=7.6,0.9 \mathrm{~Hz}, 1 \mathrm{H}), 6.96(\mathrm{~d}, J=7.7 \mathrm{~Hz}, 1 \mathrm{H}), 6.90(\mathrm{~s}, 1 \mathrm{H}), 6.85(\mathrm{dd}, J=8.3,1.8 \mathrm{~Hz}, 1 \mathrm{H}), 6.63(\mathrm{~d}, J=8.1 \mathrm{~Hz}, 1 \mathrm{H}), 6.40(\mathrm{ddd}$, $J=17.9,10.3,6.7 \mathrm{~Hz}, 1 \mathrm{H}), 6.22(\mathrm{~d}, J=6.8 \mathrm{~Hz}, 1 \mathrm{H}), 5.49(\mathrm{~d}, J=10.4 \mathrm{~Hz}, 1 \mathrm{H}), 5.44(\mathrm{~d}, J=17.1 \mathrm{~Hz}, 1 \mathrm{H}), 3.78(\mathrm{~s}, 3 \mathrm{H})$.

${ }^{13}$ C NMR (101 MHz, CDCl $): \delta 183.3$ (C), 160.2 (C), 158.2 (C), 149.9 (C), $138.0(\mathrm{CH}), 138.0(\mathrm{C}), 131.9(\mathrm{CH}), 130.1$

$(\mathrm{CH}), 125.5(\mathrm{CH}), 123.7(\mathrm{CH}), 120.7\left(\mathrm{CH}_{2}\right), 119.5(\mathrm{CH}), 118.1(\mathrm{C}), 113.4(\mathrm{CH}), 113.3(\mathrm{CH}), 113.2(\mathrm{CH}), 57.0(\mathrm{CH}), 55.4$ $\left(\mathrm{CH}_{3}\right)$.

FT-IR (thin film): 3081, 2939, 1739, 1610, 1489, 1467, 1435, 1347, 1269, 1191, 1158, 1128, 1095, 1044, 990, 939, 875, $794,756,695 \mathrm{~cm}^{-1}$.

HRMS: Calculated for $\mathrm{C}_{18} \mathrm{H}_{15} \mathrm{NO}_{3}[\mathrm{M}+\mathrm{H}]^{+} 294.1130$, found 294.1123.

HPLC: 88:12 er (Daicel CHIRALPAK IA, 97:3 heptane $/{ }^{\prime} \mathrm{PrOH}, 0.8 \mathrm{~mL} / \mathrm{min} ; T_{\text {major }}=20.9 \mathrm{~min}, T_{\text {minor }}=18.6 \mathrm{~min}$ ) $[\alpha]_{\mathbf{D}}{ }^{25}:+106.7^{\circ}\left(c=1.0, \mathrm{CHCl}_{3}\right)$
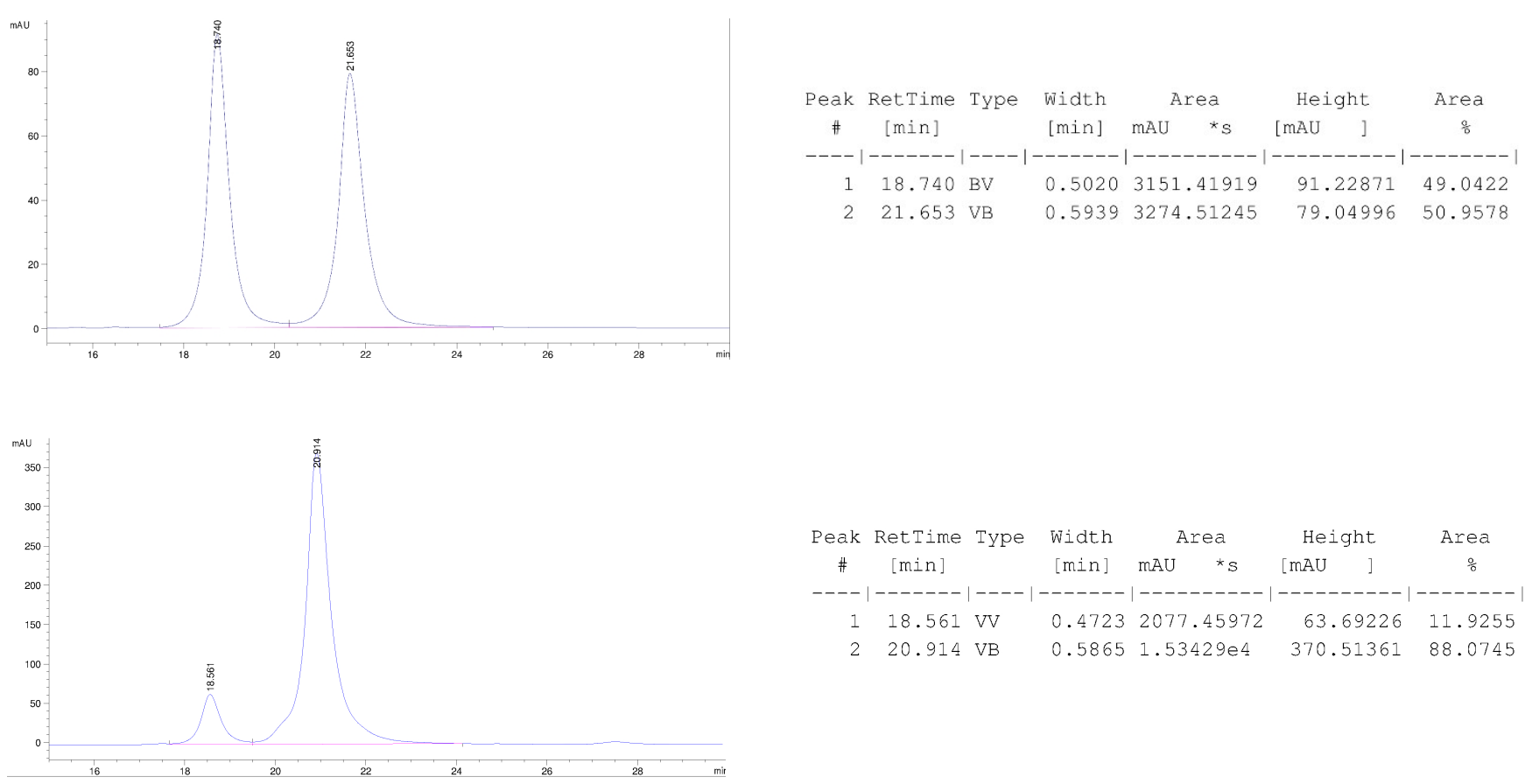


\section{1-[(R)-1-(4-Biphenylyl)-2-propenyl]-2,3-indolinedione (9ad)}

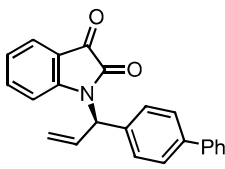

The title compound was prepared according to general procedure A using isatin $7 \mathbf{a}(73.6 \mathrm{mg}, 0.500 \mathrm{mmol}$, 1.00 equiv) and cinnamyl chloride $\mathbf{8 d}(114.4 \mathrm{mg}, 0.50 \mathrm{mmol}, 1.00$ equiv) with the following modification: the reaction vial was charged with $\mathbf{7 a}, \mathbf{8 d}$, and solvent $(50 \mu \mathrm{L})$, followed by the catalyst solution and DIPEA. After 18 hours, $42 \%$ conversion of cinnamyl chloride 8d was observed by ${ }^{1} \mathrm{H}$ NMR, so a solution of $\mathrm{CpRu}(\mathrm{MeCN})_{3} \mathrm{PF}_{6}(1.1 \mathrm{mg}, 0.0025 \mathrm{mmol}, 0.0050$ equiv) and ligand $\mathbf{L 1}$ (2.9 $\mathrm{mg}, 0.0030 \mathrm{mmol}, 0.0060$ equiv) in 3pentanone $(50 \mu \mathrm{L})$ was added and the reaction was stirred at $40{ }^{\circ} \mathrm{C}$ overnight. At this point, crude ${ }^{1} \mathrm{H}$ NMR showed full conversion of cinnamyl chloride 8d and $>20: 1$ branched/linear selectivity. Purification by flash chromatography (12 $\mathrm{g}$ silica, $5: 1 \rightarrow 1: 1$ petroleum ether/EtOAc) afforded 9 ad as a bright yellow solid. $(88 \mathrm{mg}, 52 \%)$

Physical appearance: Bright yellow solid

Melting point: $129-150{ }^{\circ} \mathrm{C}$

TLC $\mathbf{R}_{\mathbf{f}}$ (5:1 petroleum ether/EtOAc): 0.26

${ }^{1}$ H NMR (400 MHz, CDCl $)$ ): $\delta 7.59(\mathrm{~m}, 5 \mathrm{H}), 7.41(\mathrm{~m}, 6 \mathrm{H}), 7.07(\mathrm{t}, J=7.6 \mathrm{~Hz}, 1 \mathrm{H}), 6.67(\mathrm{~d}, J=8.1 \mathrm{~Hz}, 1 \mathrm{H}), 6.45(\mathrm{ddd}, J$ $=17.0,10.3,6.7 \mathrm{~Hz}, 1 \mathrm{H}), 6.30(\mathrm{~d}, J=6.8 \mathrm{~Hz}, 1 \mathrm{H}), 5.52(\mathrm{dd}, J=10.3,1.1 \mathrm{~Hz}, 1 \mathrm{H}), 5.48(\mathrm{dd}, J=17.1,1.2 \mathrm{~Hz}, 1 \mathrm{H})$.

${ }^{13}$ C NMR (101 MHz, $\left.\mathbf{C D C l}_{3}\right): \delta 183.4,158.3,149.9,141.2,140.3,138.1,135.3,132.0,129.0,127.8,127.2,125.6,123.8$, $120.8,118.1,113.4,57.0$; Two fewer aromatic signals than expected due to overlapping peaks

FT-IR (thin film): 3058, 3030, 2922, 1739, 1610, 1519, 1485, 1467, 1408, 1346, 1301, 1184, 1158, 1095, 988, 941, 910 , $847,816,754,698 \mathrm{~cm}^{-1}$.

HRMS: Calculated for $\mathrm{C}_{23} \mathrm{H}_{17} \mathrm{NO}_{2}[\mathrm{M}+\mathrm{H}]^{+} 340.1338$, found 340.1328 .

HPLC: 90:10 er (Daicel CHIRALPAK IC, 97:3 heptane $/{ }^{2} \operatorname{PrOH}, 0.8 \mathrm{~mL} / \mathrm{min}, 254 \mathrm{~nm} ; T_{\text {major }}=16.3 \mathrm{~min}, T_{\text {minor }}=15.4 \mathrm{~min}$ ) $[\alpha]_{\mathbf{D}}{ }^{25}:+71.5^{\circ}\left(c=1.0, \mathrm{CHCl}_{3}\right)$
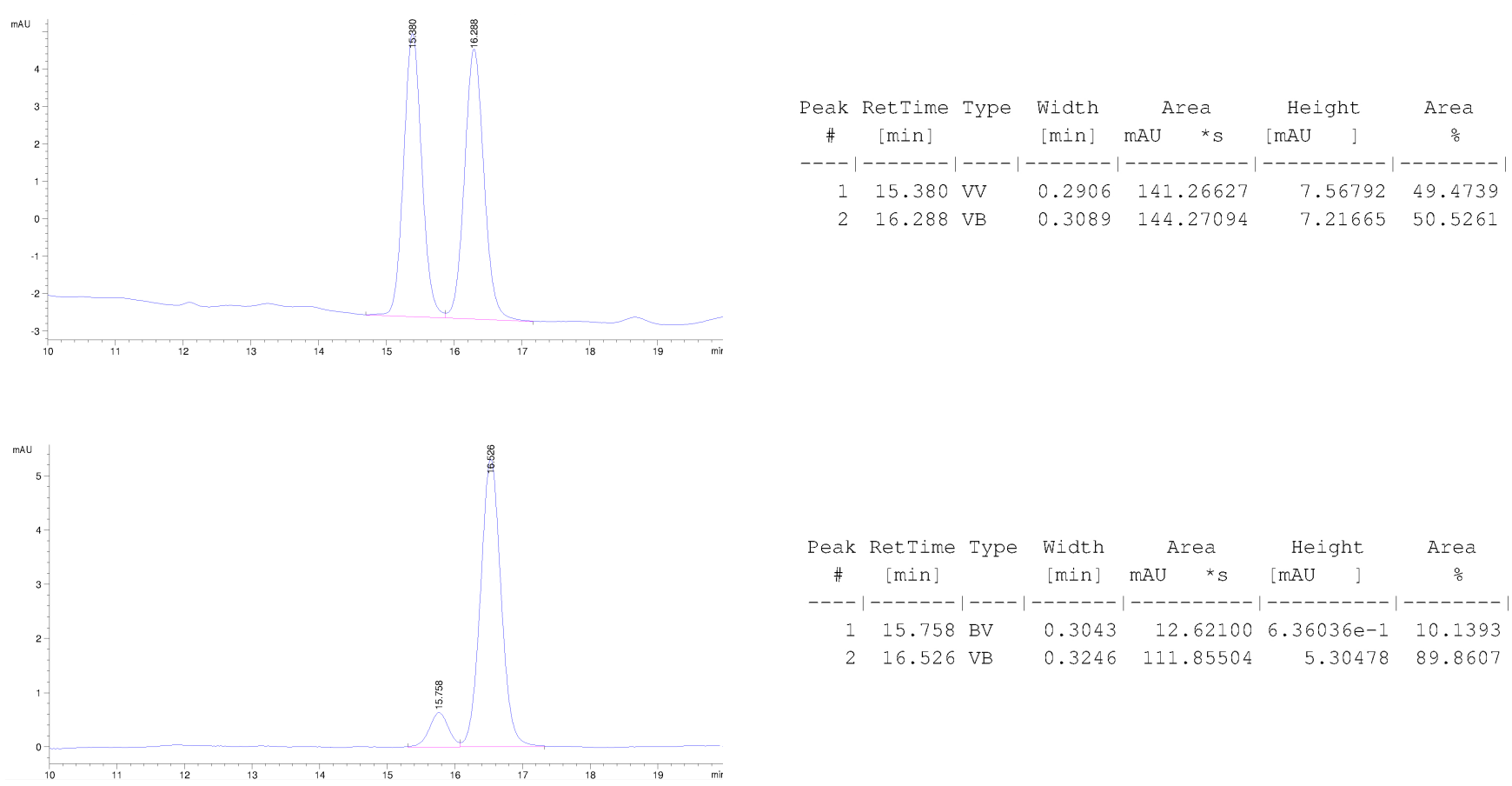

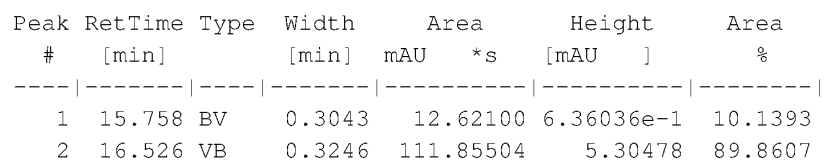




\section{1-[(R)-1-(6-Methoxy-2-naphthyl)-2-propenyl]-5-chloro-2,3-indolinedione (9de)}

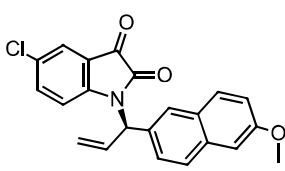

The title compound was prepared according to general procedure A using 5-chloroisatin $7 \mathbf{d}(90.8 \mathrm{mg}$, $0.500 \mathrm{mmol}, 1.00$ equiv) and cinnamyl chloride $8 \mathrm{e}(116 \mathrm{mg}, 0.50 \mathrm{mmol}, 1.00$ equiv) with the

following modification: the reaction vial was charged with $\mathbf{7 d}, \mathbf{8 e}$, and solvent $(50 \mu \mathrm{L})$, followed by the catalyst solution and DIPEA. After 19 hours, $67 \%$ conversion of cinnamyl chloride 8e was observed by ${ }^{1} \mathrm{H} \mathrm{NMR}$, so a solution of $\mathrm{CpRu}(\mathrm{MeCN})_{3} \mathrm{PF}_{6}(1.1 \mathrm{mg}, 0.0025 \mathrm{mmol}, 0.0050$ equiv) and ligand $\mathbf{L 1}(2.9 \mathrm{mg}, 0.0030 \mathrm{mmol}$, 0.0060 equiv) in 3-pentanone $(50 \mu \mathrm{L})$ was added and the reaction was stirred at $40{ }^{\circ} \mathrm{C}$ overnight. At this point, crude ${ }^{1} \mathrm{H}$ NMR showed full conversion of cinnamyl chloride 8e and 6:1 branched/linear selectivity. Purification by flash chromatography (13 g silica, 4:1 petroleum ether/EtOAc $\rightarrow$ EtOAc) afforded 9de as an orange oil. (94 mg, 49\%)

Physical appearance: Orange oil

TLC $\mathbf{R}_{\mathbf{f}}$ (3:1 petroleum ether/EtOAc): 0.24

${ }^{1}$ H NMR (400 MHz, $\left.\mathbf{C D C l}_{3}\right): 7.75(\mathrm{~s}, 1 \mathrm{H}), 7.72(\mathrm{~d}, J=3.1 \mathrm{~Hz}, 1 \mathrm{H}), 7.70(\mathrm{~d}, J=3.6 \mathrm{~Hz}, 1 \mathrm{H}), 7.53(\mathrm{~d}, J=2.2 \mathrm{~Hz}, 1 \mathrm{H}), 7.35$ $(\mathrm{dd}, J=8.6,1.9 \mathrm{~Hz}, 1 \mathrm{H}), 7.22(\mathrm{dd}, J=8.5,2.3 \mathrm{~Hz}, 1 \mathrm{H}), 7.17(\mathrm{dd}, J=8.9,2.6 \mathrm{~Hz}, 1 \mathrm{H}), 7.11(\mathrm{~d}, J=2.6 \mathrm{~Hz}, 1 \mathrm{H}), 6.57(\mathrm{~d}, J=$ $8.6 \mathrm{~Hz}, 1 \mathrm{H}), 6.48(\mathrm{ddd}, J=16.8,10.3,6.5 \mathrm{~Hz}, 1 \mathrm{H}), 6.38(\mathrm{dd}, J=6.6,1.4 \mathrm{~Hz}, 1 \mathrm{H}), 5.55(\mathrm{~d}, J=10.2 \mathrm{~Hz}, 1 \mathrm{H}), 5.47$ (dd, $J=$ $17.0,1.2 \mathrm{~Hz}, 1 \mathrm{H}), 3.90(\mathrm{~s}, 3 \mathrm{H})$.

${ }^{13}$ C NMR (101 MHz, CDCl $)$ ): $\delta 182.4(\mathrm{C}), 158.4(\mathrm{C}), 157.8(\mathrm{C}), 148.2(\mathrm{C}), 137.3(\mathrm{CH}), 134.3(\mathrm{C}), 131.6(\mathrm{CH}), 131.0(\mathrm{C})$, $129.6(\mathrm{C}), 129.6(\mathrm{CH}), 128.7(\mathrm{C}), 128.0(\mathrm{CH}), 126.2(\mathrm{CH}), 125.3(\mathrm{CH}), 125.3(\mathrm{CH}), 120.7\left(\mathrm{CH}_{2}\right), 119.7(\mathrm{CH}), 119.0(\mathrm{C})$, $114.6(\mathrm{CH}), 105.8(\mathrm{CH}), 57.2(\mathrm{CH}), 55.5\left(\mathrm{CH}_{3}\right)$.

FT-IR (thin film): 3062, 2005, 2959, 2938, 2842, 1744, 1609, 1505, 1467, 1444, 1391, 1369, 1319, 1268, 1219, 1193, $1176,1127,1070,1031,992,908,855,820,777,762,732,687,648 \mathrm{~cm}^{-1}$.

HRMS: Calculated for $\mathrm{C}_{22} \mathrm{H}_{16} \mathrm{ClNO}_{3}[\mathrm{M}+\mathrm{Na}]^{+} 400.0716$, found 400.0707 .

HPLC: $81: 19 \mathrm{er}$ (Daicel CHIRALPAK OJ-H, 80:20 heptane $/ \mathrm{PrOH}, 1.0 \mathrm{~mL} / \mathrm{min}, 254 \mathrm{~nm} ; T_{\text {major }}=88.7 \mathrm{~min}, T_{\text {minor }}=131.4 \mathrm{~min}$ ) $[\alpha]_{D}^{25}:+76.9^{\circ}\left(c=1.0, \mathrm{CHCl}_{3}\right)$
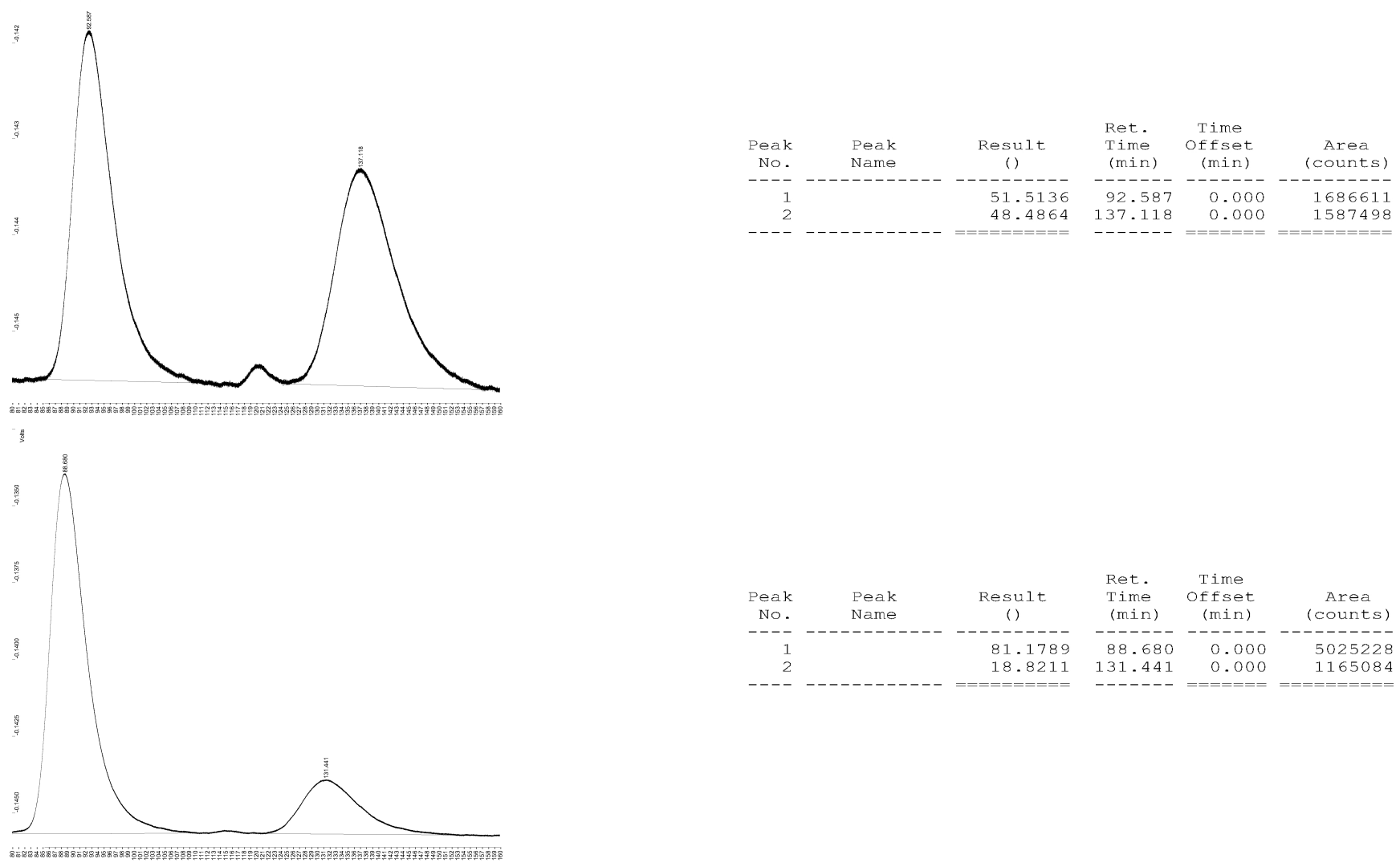


\section{1-[(S)-1-Methyl-2-propenyl]-2,3-indolinedione (9af)}

$\mathrm{C}_{\mathrm{CH}}=\mathrm{O}$

The title compound was prepared according to general procedure A using isatin $7 \mathbf{a}(73.6 \mathrm{mg}, 0.500 \mathrm{mmol}, 1.00$ equiv) and crotyl chloride $8 \mathbf{8}$ ( $48.7 \mu \mathrm{L}, 0.50 \mathrm{mmol}, 1.00$ equiv). Crude ${ }^{1} \mathrm{H}$ NMR showed 17:1 branched/linear selectivity. Purification by flash chromatography (4 $\mathrm{g}$ silica, 5:1 petroleum ether/EtOAc) afforded 9af as a bright orange-red solid. (78 $\mathrm{mg}, 78 \%$ )

Physical appearance: Bright orange-red solid

Melting point: $53-67^{\circ} \mathrm{C}$

TLC $R_{\mathbf{f}}$ (5:1 petroleum ether/EtOAc): 0.10

${ }^{1}$ H NMR (300 MHz, CDCl $\left.)_{3}\right): \delta 7.61(\mathrm{~m}, 1 \mathrm{H}), 7.53(\mathrm{td}, J=7.8,1.5 \mathrm{~Hz}, 1 \mathrm{H}), 7.10(\mathrm{td}, J=7.6,0.7 \mathrm{~Hz}, 1 \mathrm{H}), 7.02(\mathrm{~d}, J=8.0$ $\mathrm{Hz}, 1 \mathrm{H}), 6.05$ (ddd, $J=17.5,10.5,4.4 \mathrm{~Hz}, 1 \mathrm{H}), 5.33(\mathrm{~m}, 2 \mathrm{H}), 5.15(\mathrm{~m}, 1 \mathrm{H}), 1.59$ (d, $J=7.0 \mathrm{~Hz}, 3 \mathrm{H})$.

${ }^{13}$ C NMR (101 MHz, $\left.\mathbf{C D C l}_{3}\right): \delta 183.6,157.8,150.0,138.0,136.0,125.6,123.6,118.0,117.5,112.6,49.1,16.2$.

FT-IR (thin film): 3087, 2983, 1744, 1611, 1468, 1416, 1377, 1349, 1306, 1201, 1159, 1094, 1062, 978, 931, 889, 818, $756,697,662 \mathrm{~cm}^{-1}$.

HRMS: Calculated for $\mathrm{C}_{12} \mathrm{H}_{11} \mathrm{NO}_{2}[\mathrm{M}+\mathrm{H}]^{+}$202.0868, found 202.0863.

HPLC: $85: 15$ er (Daicel CHIRALPAK AD-H, 97:3 heptane $/ / \mathrm{PrOH}, 1.0 \mathrm{~mL} / \mathrm{min} ; T_{\text {major }}=19.4 \mathrm{~min}, T_{\text {minor }}=18.3 \mathrm{~min}$ ) $[\alpha]_{\mathrm{D}}{ }^{24}:-8.7^{\circ}\left(89: 11 \mathrm{er}, c=1.43, \mathrm{CHCl}_{3}\right)$
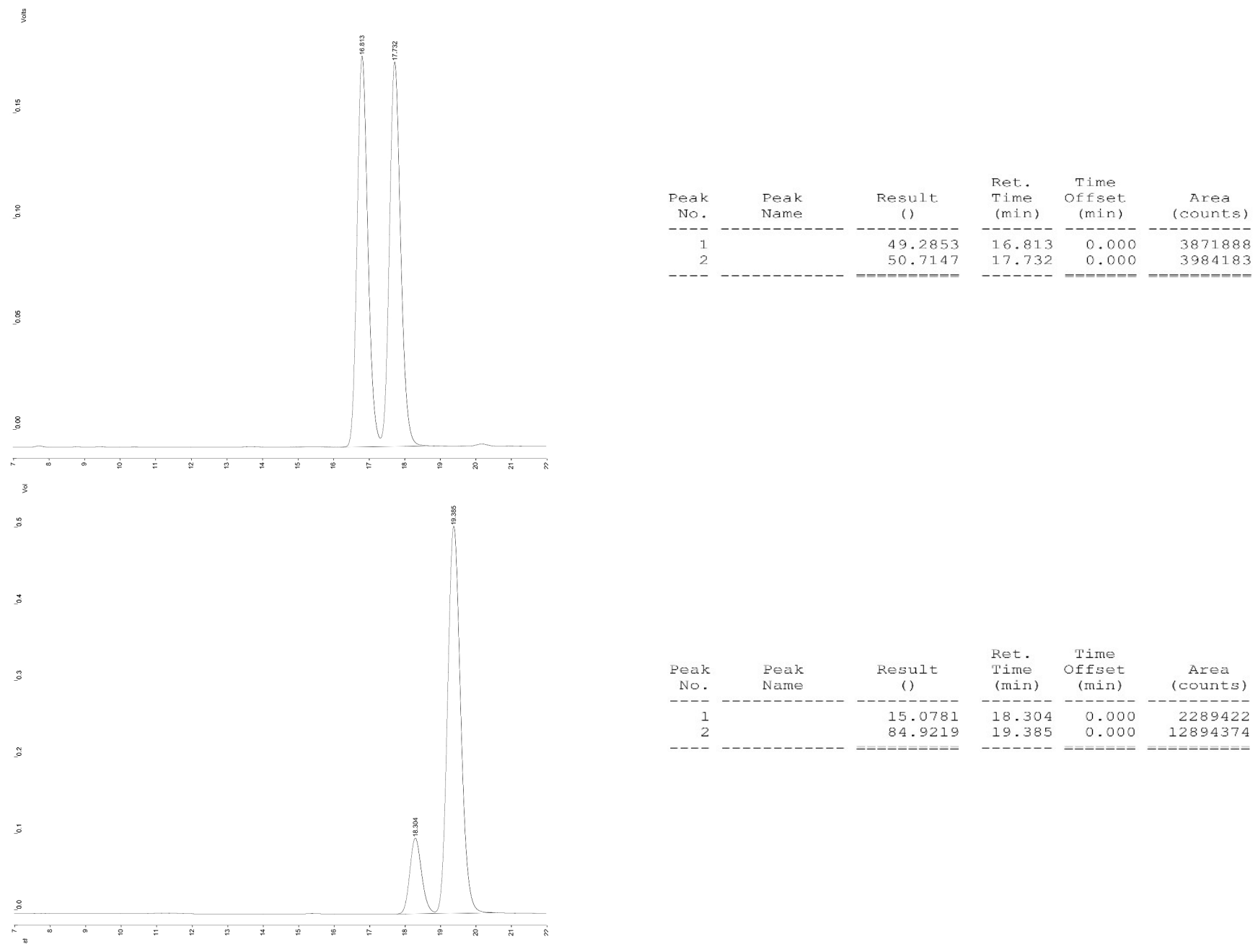


\section{1-[(R)-4,4-Dimethyl-1-vinyl-2-pentynyl]-5-methyl-2,3-indolinedione (9mg)}

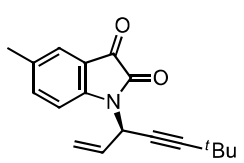

The title compound was prepared according to general procedure B using 5-methylisatin $7 \mathbf{m}(80.6 \mathrm{mg}$, $0.500 \mathrm{mmol}, 1.00$ equiv) and 1-chloro-6,6-dimethyl-2-hepten-4-yne $8 \mathrm{~g}$ (78 $\mathrm{mg}, 0.50 \mathrm{mmol}, 1.00$ equiv). Crude ${ }^{1} \mathrm{H}$ NMR showed 4:1 branched/linear selectivity. Purification by flash chromatography (5 g silica, 9:1 $\rightarrow$ 5:1 petroleum ether/EtOAc) afforded $9 \mathrm{mg}$ as an orange solid. (56 $\mathrm{mg}, 40 \%)$

Physical appearance: Bright orange-red solid

Melting point: Changes appearance at $59^{\circ} \mathrm{C}$; completely melts by $123{ }^{\circ} \mathrm{C}$

TLC $\mathbf{R}_{\mathbf{f}}$ (5:1 petroleum ether/EtOAc): 0.10

${ }^{1}$ H NMR (400 MHz, CDCl $): \delta 7.43(\mathrm{~s}, 1 \mathrm{H}), 7.34(\mathrm{~m}, 1 \mathrm{H}), 7.11(\mathrm{~d}, J=8.2 \mathrm{~Hz}, 1 \mathrm{H}), 5.93(\mathrm{dt}, J=4.0,2.0 \mathrm{~Hz}, 1 \mathrm{H}), 5.83(\mathrm{ddd}$, $J=16.8,9.8,3.8 \mathrm{~Hz}, 1 \mathrm{H}), 5.68$ (ddd, $J=16.9,2.0,1.1 \mathrm{~Hz}, 1 \mathrm{H}), 5.42$ (ddd, $J=9.8,2.2,1.0 \mathrm{~Hz}, 1 \mathrm{H}), 2.33(\mathrm{~s}, 3 \mathrm{H}), 1.23(\mathrm{~s}, 9 \mathrm{H})$.

${ }^{13}$ C NMR (101 MHz, $\left.\mathbf{C D C l}_{3}\right): \delta 183.4(\mathrm{C}), 157.2(\mathrm{C}), 147.2(\mathrm{C}), 138.3(\mathrm{CH}), 133.6(\mathrm{C}), 132.6(\mathrm{CH}), 125.5(\mathrm{CH}), 119.3$ $\left(\mathrm{CH}_{2}\right), 118.1(\mathrm{C}), 113.3(\mathrm{CH}), 97.2(\mathrm{C}), 71.4(\mathrm{C}), 44.9(\mathrm{CH}), 30.8\left(\mathrm{CH}_{3}\right), 27.7(\mathrm{C}), 20.8\left(\mathrm{CH}_{3}\right)$.

FT-IR (thin film): 3090, 3024, 2970, 2928, 2868, 2243, 1745, 1622, 1595, 1489, 1453, 1401, 1367, 1326, 1262, 1194 , $1130,1014,987,935,894,820,791,747,717,679,626 \mathrm{~cm}^{-1}$.

HRMS: Calculated for $\mathrm{C}_{18} \mathrm{H}_{19} \mathrm{NO}_{2}[\mathrm{M}+\mathrm{H}]^{+} 282.1494$, found 282.1491 .

HPLC: $82: 18$ er (Daicel CHIRALPAK OJ-H, 95:5 heptane $/{ }^{i} \mathrm{PrOH}, 1.0 \mathrm{~mL} / \mathrm{min} ; T_{\text {major }}=9.4 \mathrm{~min}, T_{\text {minor }}=10.5 \mathrm{~min}$ ) $[\boldsymbol{\alpha}]_{\mathrm{D}}{ }^{24}:-113.3^{\circ}\left(c=1.0, \mathrm{CHCl}_{3}\right)$
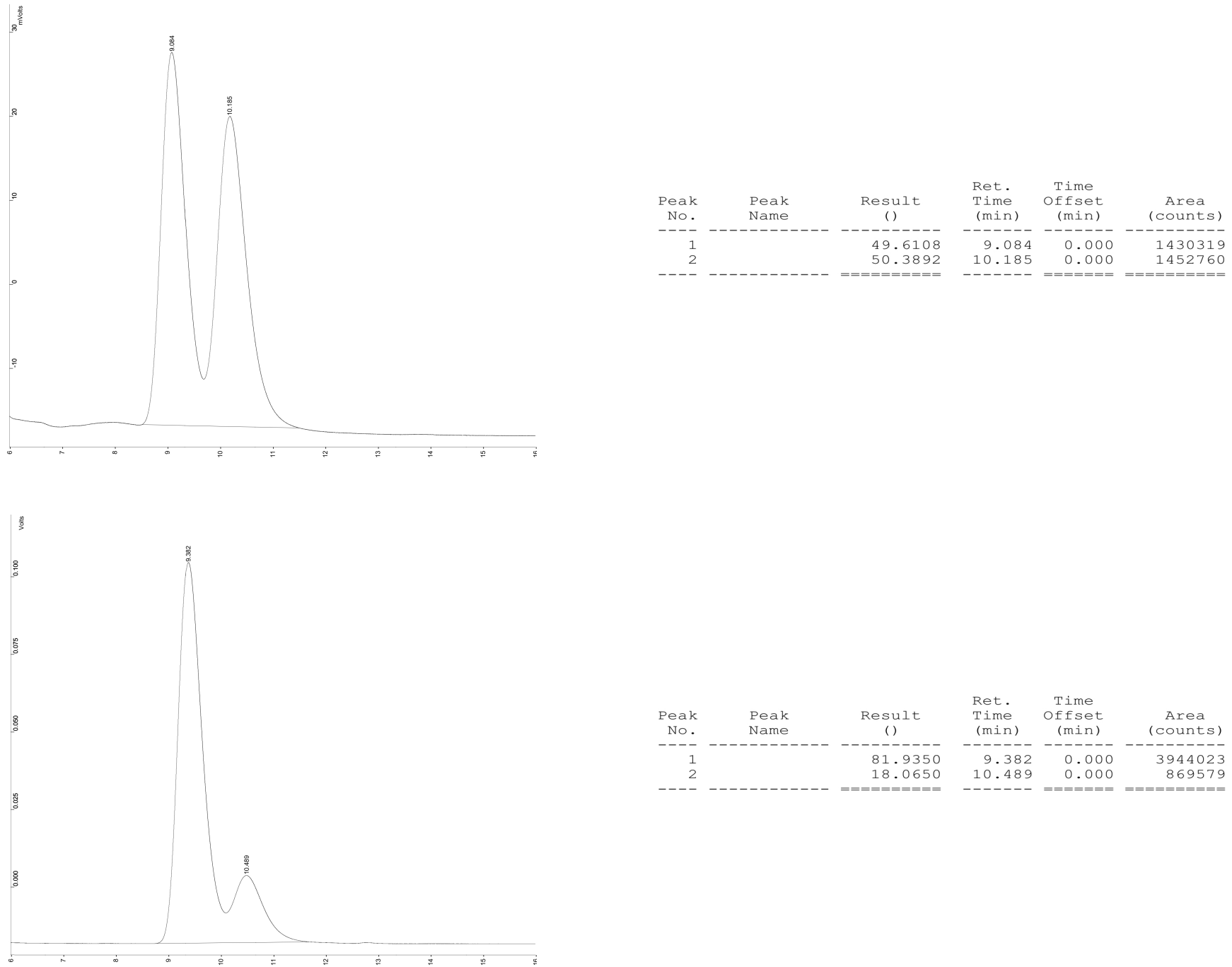


\section{5-[(S)-2-(5-Methyl-2,3-dioxo-1-indolinyl)-3-butenyl]-2,2,5-trimethyl-1,3-dioxane-4,6-dione (9mh)}

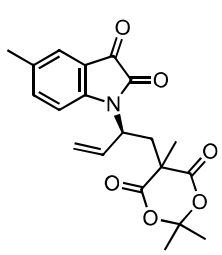

The title compound was prepared according to general procedure B using 5-methylisatin $\mathbf{7 m}(80.8 \mathrm{mg}$, $0.500 \mathrm{mmol}, 1.00$ equiv) and 5-[(E)-4-bromo-2-butenyl]-2,2,5-trimethyl-1,3-dioxane-4,6-dione $8 \mathbf{h}$ (146 $\mathrm{mg}, 0.50 \mathrm{mmol}, 1.00$ equiv) with the following modification: the reaction vial was charged with $\mathbf{7} \mathbf{m}, \mathbf{8 h}$, and solvent $(50 \mu \mathrm{L})$, followed by the catalyst solution and DIPEA. Crude ${ }^{1} \mathrm{H}$ NMR showed 2.5:1 branched/linear selectivity and $80 \%$ conversion based on allylic bromide $\mathbf{8 h}$. Purification by flash chromatography (10 g silica, 30:1 $\rightarrow$ 5:1 $\mathrm{CH}_{2} \mathrm{Cl}_{2} / \mathrm{EtOAc}$ ) afforded $9 \mathbf{m h}$ as an orange solid. (66 $\mathrm{mg}, 35 \%$ )

Physical appearance: Orange solid

Melting point: $118-135^{\circ} \mathrm{C}$

TLC R $\mathbf{R}_{\mathbf{f}}$ (30:1 $\left.\mathrm{CH}_{2} \mathrm{Cl}_{2} / \mathrm{EtOAc}\right): 0.32$

${ }^{1}$ H NMR (400 MHz, $\left.\mathbf{C D C l}_{3}\right): \delta 7.43(\mathrm{~m}, 1 \mathrm{H}), 7.37(\mathrm{~m}, 1 \mathrm{H}), 6.86(\mathrm{~d}, J=8.2 \mathrm{~Hz}, 1 \mathrm{H}), 5.97(\mathrm{ddd}, J=17.3,10.6,5.4 \mathrm{~Hz}, 1 \mathrm{H})$, $5.33(\mathrm{ddd}, J=5.7,1.9,0.5 \mathrm{~Hz}, 1 \mathrm{H}), 5.30$ (ddd, $J=12.5,1.9,0.5 \mathrm{~Hz}, 1 \mathrm{H}), 5.00$ (dddd, $J=11.3,7.4,4.0,1.8 \mathrm{~Hz}, 1 \mathrm{H}), 2.90$ $(\mathrm{dd}, J=14.2,10.8 \mathrm{~Hz}, 1 \mathrm{H}), 2.66(\mathrm{dd}, J=14.2,4.2 \mathrm{~Hz}, 1 \mathrm{H}), 2.33(\mathrm{~s}, 3 \mathrm{H}), 1.71(\mathrm{~s}, 3 \mathrm{H}), 1.70$ (s, 3H), 1.68 (s, 3H).

${ }^{13}$ C NMR (101 MHz, $\left.\mathbf{C D C l}_{3}\right): \delta 182.7$ (C), $169.0(\mathrm{C}), 168.9(\mathrm{C}), 158.5(\mathrm{C}), 147.2(\mathrm{C}), 138.5(\mathrm{CH}), 133.6(\mathrm{C}), 133.4(\mathrm{CH})$, $125.9(\mathrm{CH}), 119.3\left(\mathrm{CH}_{2}\right), 118.1(\mathrm{C}), 112.8(\mathrm{CH}), 106.0(\mathrm{C}), 50.8(\mathrm{CH}), 46.8(\mathrm{C}), 40.3\left(\mathrm{CH}_{2}\right), 29.9\left(\mathrm{CH}_{3}\right), 27.7\left(\mathrm{CH}_{3}\right), 26.1$ $\left(\mathrm{CH}_{3}\right), 20.7\left(\mathrm{CH}_{3}\right)$.

FT-IR (thin film): 3088, 2994, 2945, 2923, 1731, 1623, 1596, 1490, 1457, 1424, 1388, 1334, 1296, 1201, 1154, 1059, 979, $919,825,786,733,700,647 \mathrm{~cm}^{-1}$.

HRMS: Calculated for $\mathrm{C}_{20} \mathrm{H}_{21} \mathrm{NO}_{6}[\mathrm{M}+\mathrm{Na}]^{+} 394.1267$, found 394.1266.

HPLC: 95:5 er (Daicel CHIRALPAK OJ-H, 80:20 heptane $/{ }^{i} \mathrm{PrOH}, 1.0 \mathrm{~mL} / \mathrm{min} ; T_{\text {major }}=64.5 \mathrm{~min}, T_{\text {minor }}=48.2 \mathrm{~min}$ ) $[\alpha]_{\mathbf{D}}{ }^{25}:+65.2^{\circ}\left(c=1.0, \mathrm{CHCl}_{3}\right)$
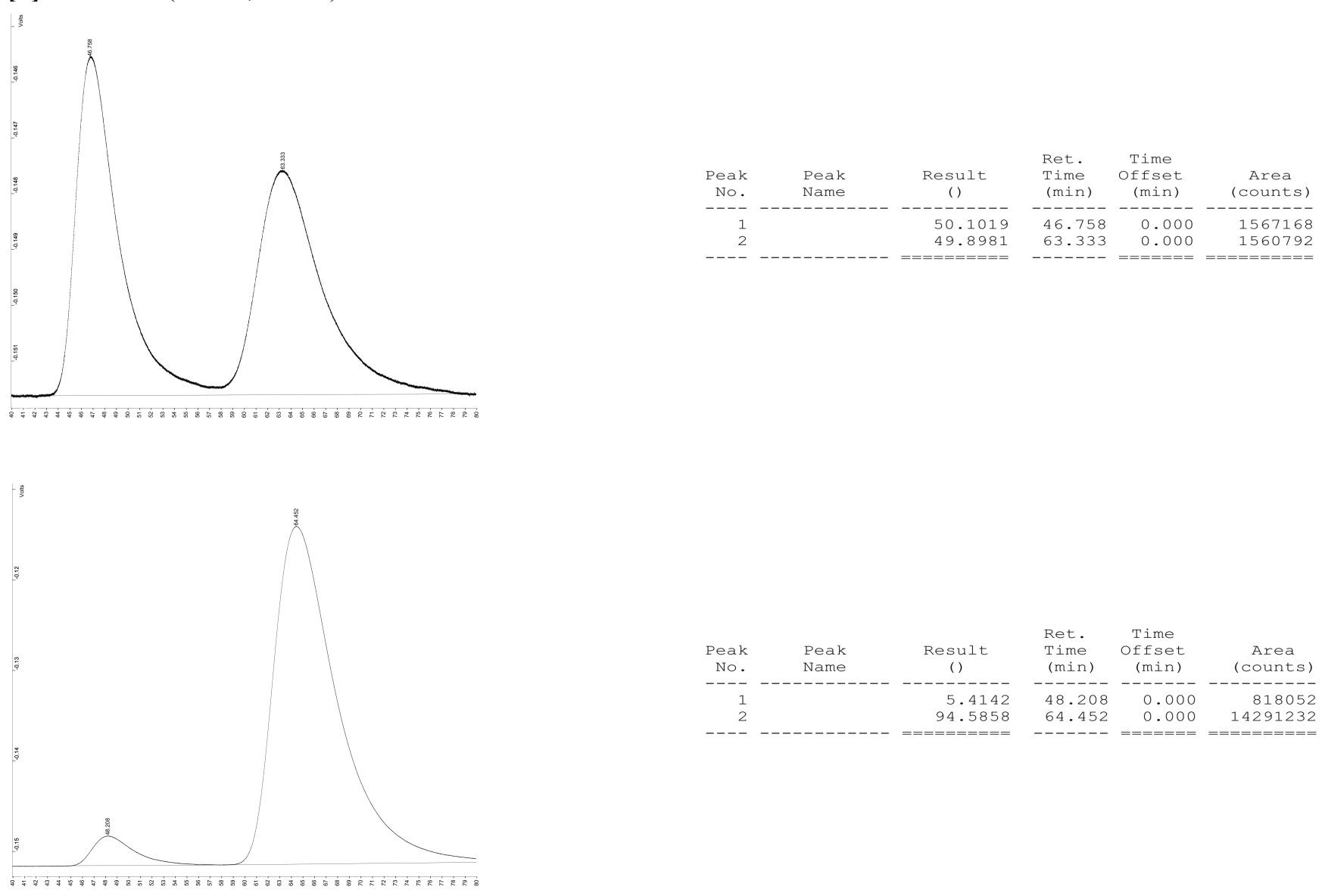
(R)-2-(5-Iodo-2,3-dioxo-1-indolinyl)-3-butenyl 2,2-dimethylpropionate (9ei)

$C_{N}=0$

The title compound was prepared according to general procedure B using 5-iodoisatin $7 \mathbf{e}(136.5 \mathrm{mg}, 0.500$ mmol, 1.00 equiv) and bromide $8 \mathbf{i}$ (118 $\mathrm{mg}, 0.500 \mathrm{mmol}, 1.00$ equiv) with the following modification: the

reaction vial was charged with $\mathbf{7 e}, \mathbf{8 i}$, and solvent $(50 \mu \mathrm{L})$, followed by the catalyst solution and DIPEA.

Crude ${ }^{1} \mathrm{H}$ NMR showed 1.7:1 branched/linear selectivity. Purification by flash chromatography (12 g silica, 5:1 $\rightarrow$ 1:1 petroleum ether/EtOAc) afforded 9ei as an orange oil. (112 mg, 53\%)

Physical appearance: Orange oil

TLC $\mathbf{R}_{\mathbf{f}}$ (4:1 petroleum ether/EtOAc, $\left.\mathbf{U V}\right): 0.24$

${ }^{1}$ H NMR (400 MHz, CDCl $)$ ): $\delta 7.91(\mathrm{~d}, J=1.9 \mathrm{~Hz}, 1 \mathrm{H}), 7.85(\mathrm{dd}, J=8.4,1.5 \mathrm{~Hz}, 1 \mathrm{H}), 6.84(\mathrm{~d}, J=8.4 \mathrm{~Hz}, 1 \mathrm{H}), 6.05$ (ddd, $J=17.4,10.7,5.3 \mathrm{~Hz}, 1 \mathrm{H}), 5.44(\mathrm{dd}, J=10.8,1.8 \mathrm{~Hz}, 1 \mathrm{H}), 5.39(\mathrm{dd}, J=17.5,1.9 \mathrm{~Hz}, 1 \mathrm{H}), 5.18(\mathrm{dtt}, J=9.3,5.4,1.9 \mathrm{~Hz}$, $1 \mathrm{H}), 4.56(\mathrm{dd}, J=11.5,9.4 \mathrm{~Hz}, 1 \mathrm{H}), 4.49(\mathrm{dd}, J=11.5,5.5 \mathrm{~Hz}, 1 \mathrm{H}), 1.08(\mathrm{~s}, 9 \mathrm{H})$.

${ }^{13}$ C NMR (101 MHz, CDCl $): \delta 181.5$ (C), $178.1(\mathrm{C}), 157.3$ (C), 149.4 (C), $146.3(\mathrm{CH}), 134.1(\mathrm{CH}), 130.1(\mathrm{CH}), 120.4$ $\left(\mathrm{CH}_{2}\right), 119.5(\mathrm{C}), 114.2(\mathrm{CH}), 86.3(\mathrm{C}), 61.5\left(\mathrm{CH}_{2}\right), 53.6(\mathrm{CH}), 38.8(\mathrm{C}), 27.1\left(\mathrm{CH}_{3}\right)$.

FT-IR (thin film): 3087, 2973, 2934, 2873, 1745, 1603, 1547, 1465, 1431, 1397, 1368, 1331, 1284, 1146, 1037, 991, 939, $856,823,769,733 \mathrm{~cm}^{-1}$.

HRMS: Calculated for $\mathrm{C}_{17} \mathrm{H}_{18} \mathrm{INO}_{4}[\mathrm{M}+\mathrm{H}]^{+} 428.0359$, found 428.0355 .

HPLC: $93: 7$ er (Daicel CHIRALPAK OJ-H, 90:10 heptane $/ i \mathrm{PrOH}, 1.0 \mathrm{~mL} / \mathrm{min} ; T_{\text {major }}=48.3 \mathrm{~min}, T_{\text {minor }}=38.3 \mathrm{~min}$ ) $[\alpha]_{\mathrm{D}}{ }^{25}:-4.3^{\circ}\left(c=1.0, \mathrm{CHCl}_{3}\right)$

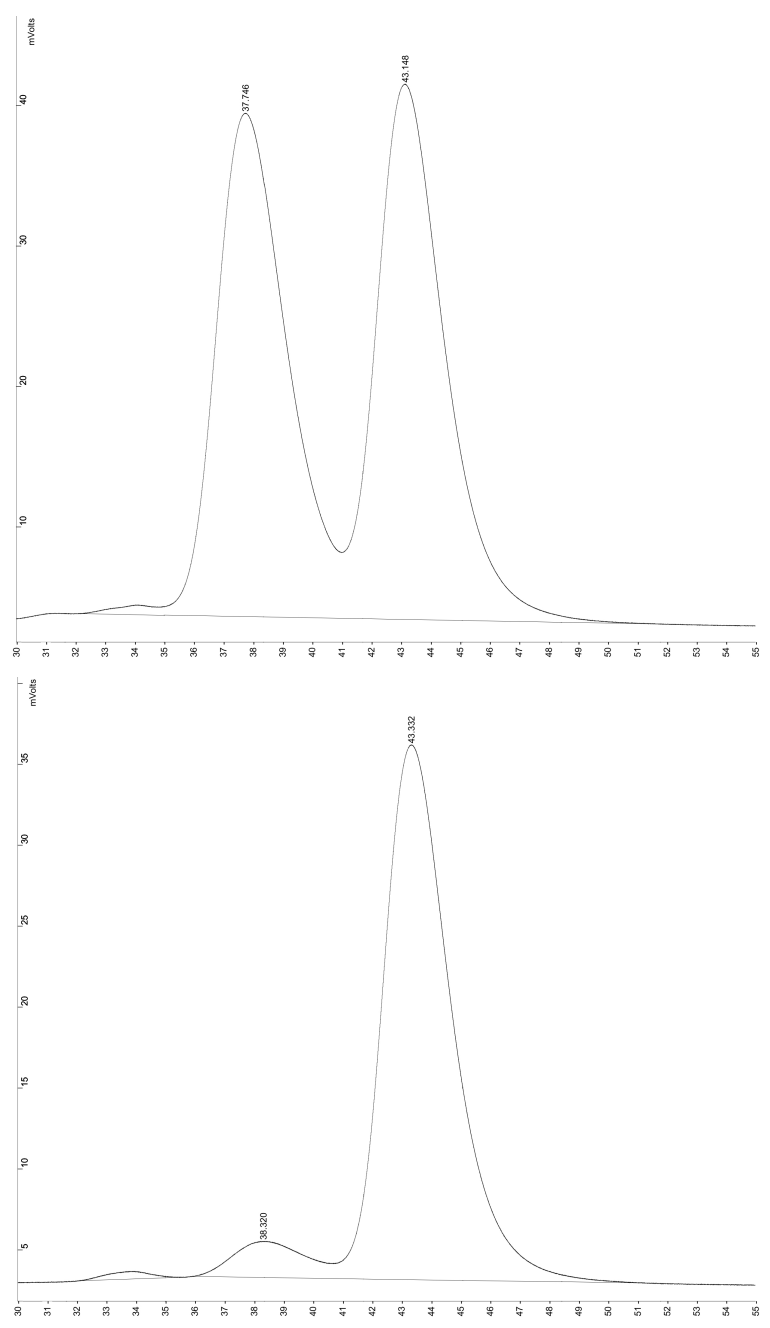

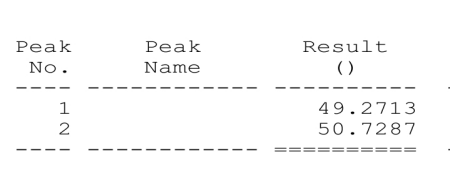
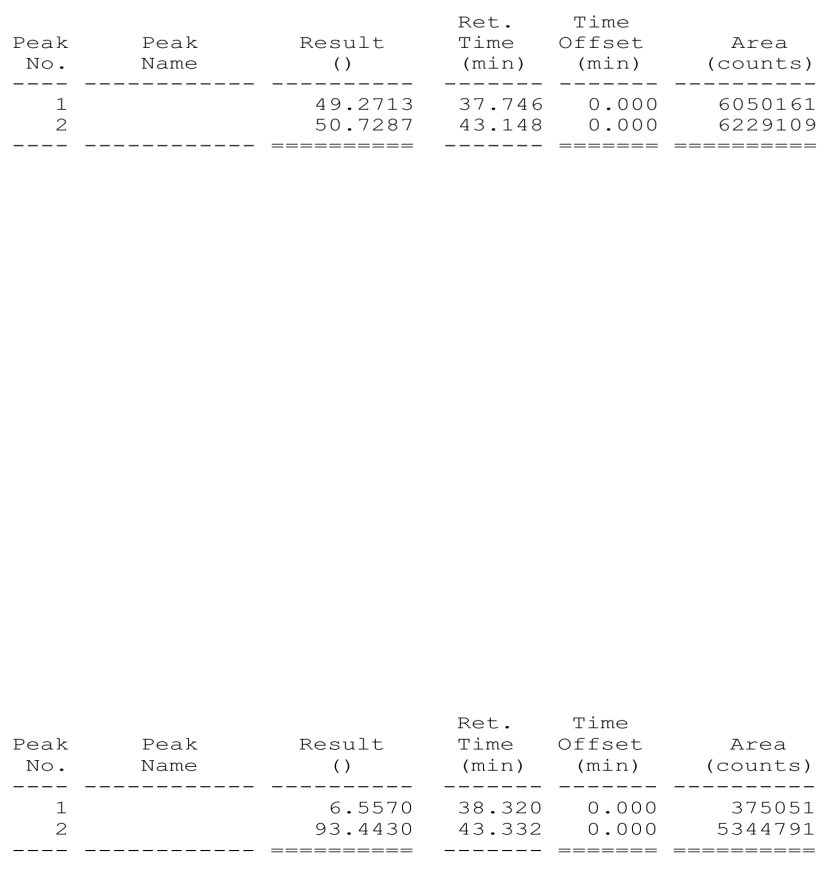


\section{1-\{(R)-1-[(3-Oxo-2-isoindolinoyl)methyl]-2-propenyl\}-5-methyl-2,3-indolinedione (9mj)}

The title compound was prepared according to general procedure B using 5-methylisatin $7 \mathbf{m}(80.8 \mathrm{mg}$,
$0.500 \mathrm{mmol}, 1.00$ equiv) and allylic bromide $\mathbf{8 j}(140 \mathrm{mg}, 0.50 \mathrm{mmol}, 1.00$ equiv) with the following
modification: the reaction vial was charged with $\mathbf{7 m}, \mathbf{8 j}$, and solvent $(50 \mu \mathrm{L})$, followed by the catalyst solution then DIPEA. Crude ${ }^{1} \mathrm{H}$ NMR showed 4:1 branched/linear selectivity. Purification by flash chromatography (12 g silica, $40: 1 \rightarrow 5: 1 \mathrm{CH}_{2} \mathrm{Cl}_{2} / \mathrm{EtOAc}$ ) afforded $9 \mathrm{mj}$ as an orange solid. (117 $\mathrm{mg}, 65 \%$ )

Physical appearance: Orange solid

Melting point: $165-168^{\circ} \mathrm{C}$

TLC $\mathbf{R}_{\mathbf{f}}\left(\mathbf{3 0 : 1} \mathbf{C H}_{2} \mathrm{Cl}_{2} / \mathrm{EtOAc}, \mathrm{UV}\right): 0.32$

${ }^{1} \mathbf{H}$ NMR (400 MHz, CDCl $): \delta 7.78(\mathrm{dd}, J=5.5,3.1 \mathrm{~Hz}, 2 \mathrm{H}), 7.69(\mathrm{dd}, J=5.5,3.0 \mathrm{~Hz}, 2 \mathrm{H}), 7.40(\mathrm{~s}, 1 \mathrm{H}), 7.36(\mathrm{~d}, J=8.1$ $\mathrm{Hz}, 1 \mathrm{H}), 7.00(\mathrm{~d}, J=8.1 \mathrm{~Hz}, 1 \mathrm{H}), 6.21(\mathrm{ddd}, J=17.1,10.6,5.9 \mathrm{~Hz}, 1 \mathrm{H}), 5.42(\mathrm{~m}, 2 \mathrm{H}), 5.21(\mathrm{dtt}, J=9.2,5.8,1.7 \mathrm{~Hz}, 1 \mathrm{H})$, $4.27(\mathrm{dd}, J=13.9,9.1 \mathrm{~Hz}, 1 \mathrm{H}), 4.21(\mathrm{~m}, 1 \mathrm{H}), 2.31(\mathrm{~s}, 3 \mathrm{H})$.

${ }^{13}$ C NMR (101 MHz, CDCl $)$ ): $\delta 182.9$ (C), 167.9 (C), 158.5 (C), 147.7 (C), $138.6(\mathrm{CH}), 134.3(\mathrm{CH}), 133.5$ (C), 131.7 (C), 131.5 $(\mathrm{CH}), 126.0(\mathrm{CH}), 123.6(\mathrm{CH}), 120.3\left(\mathrm{CH}_{2}\right), 118.0(\mathrm{C}), 111.9(\mathrm{CH}), 53.3(\mathrm{CH}), 38.0\left(\mathrm{CH}_{2}\right), 20.7\left(\mathrm{CH}_{3}\right)$.

FT-IR (thin film): 3063, 3031, 2939, 2837, 1733, 1624, 1597, 1489, 1454, 1439, 1377, 1326 1278, 1252, 1153, 1078, 1007, 935, 824, 766, 728, 701, $620 \mathrm{~cm}^{-1}$.

HRMS: Calculated for $\mathrm{C}_{21} \mathrm{H}_{16} \mathrm{~N}_{2} \mathrm{O}_{4}[\mathrm{M}+\mathrm{H}]^{+} 361.1188$, found 361.1184 .

HPLC: 98:2 er (Daicel CHIRALPAK AD-H, 90:10 heptane $/ / \mathrm{PrOH}, 1.0 \mathrm{~mL} / \mathrm{min} ; T_{\text {major }}=86.0 \mathrm{~min}, T_{\text {minor }}=78.3 \mathrm{~min}$ ) $[\alpha]_{\mathbf{D}}{ }^{25}:-106.7^{\circ}\left(c=1.0, \mathrm{CHCl}_{3}\right)$
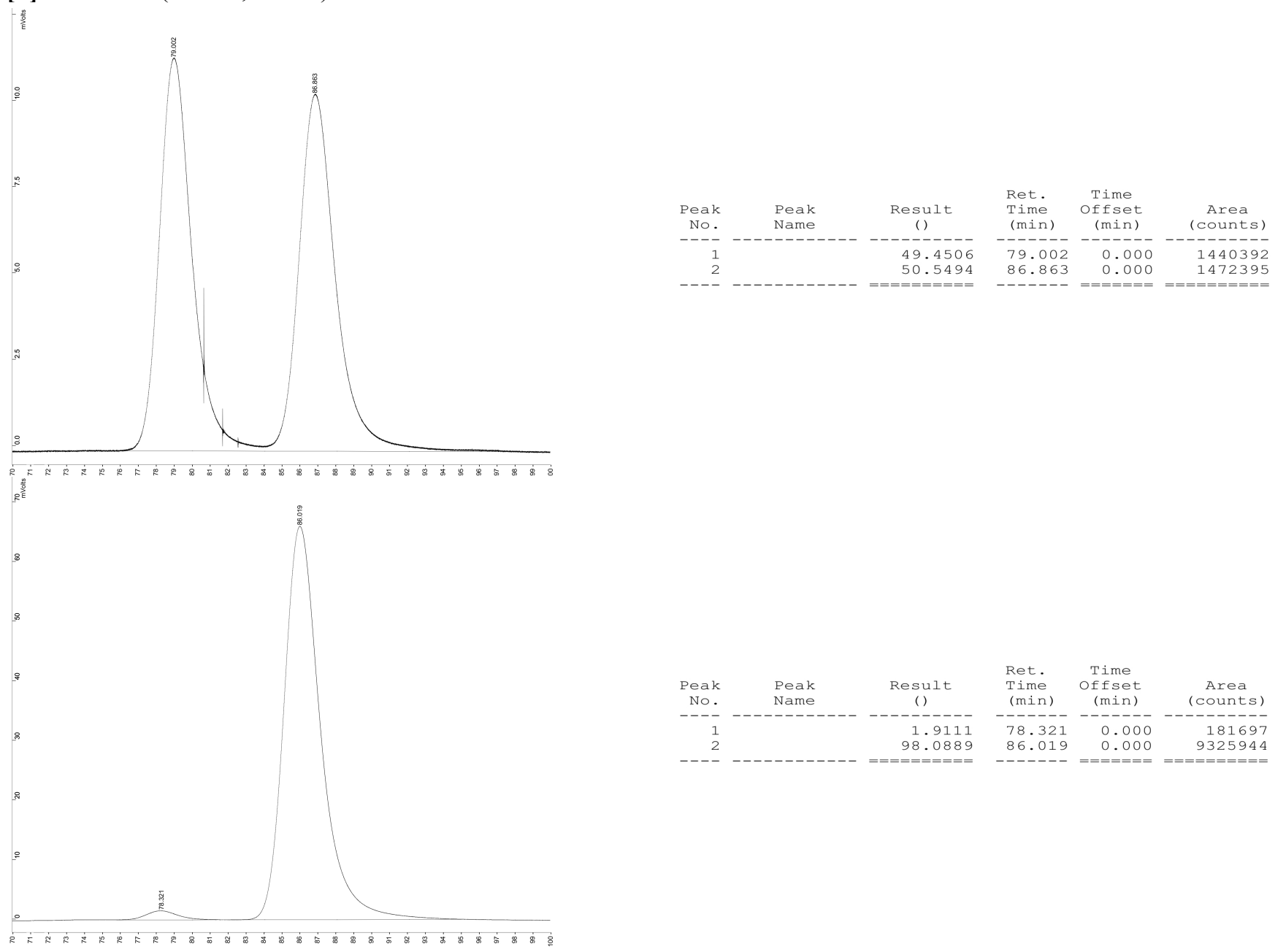


\section{Derivatization Reactions}

\section{(2-Furyl)methyltriphenylphosphonium bromide (S7)}

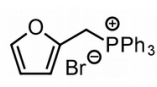

The title compound was prepared using Capuano's conditions. ${ }^{14}$ A flame-dried flask was charged with triphenylphoshonium bromide (1.888 g, $5.500 \mathrm{mmol}, 1.10$ equiv), sealed with a septum, and evacuated and backfilled with $\mathrm{N}_{2}$ three times. Anhydrous $\mathrm{MeCN}(10 \mathrm{~mL})$ and furfuryl alcohol $(0.43 \mathrm{~mL}, 5.0 \mathrm{mmol}, 1.0$ equiv) were added sequentially via syringe, and the resulting white suspension was placed in a preheated $82{ }^{\circ} \mathrm{C}$ bath. The reaction became homogeneous, then turned heterogeneous again after approximately 15 minutes. After 2 hours at $82{ }^{\circ} \mathrm{C}$, the reaction was cooled to room temperature and the precipitate was collected by vacuum filtration, rinsed with $\mathrm{Et}_{2} \mathrm{O}(4 \times 5 \mathrm{~mL})$, and dried in vacuo to afford an off-white powder that retained a significant amount of $\mathrm{MeCN}$. Drying in vacuo at $50{ }^{\circ} \mathrm{C}$ overnight afforded solvent-free material $(1.39 \mathrm{~g}, 66 \%)$. The ${ }^{1} \mathrm{H}$ NMR data match the literature. ${ }^{15}$

Physical appearance: Off-white solid

${ }^{1} \mathbf{H}$ NMR (400 MHz, $\left.\mathbf{C D C l}_{3}\right): \delta 7.76(\mathrm{~m}, 9 \mathrm{H}), 7.66(\mathrm{~m}, 6 \mathrm{H}), 7.20(\mathrm{ddd}, J=2.9,1.8,0.9 \mathrm{~Hz}, 1 \mathrm{H}), 6.54(\mathrm{t}, J=3.8 \mathrm{~Hz}, 1 \mathrm{H})$, $6.25(\mathrm{t}, J=2.5 \mathrm{~Hz}, 1 \mathrm{H}), 5.60(\mathrm{~d}, J=13.2 \mathrm{~Hz}, 2 \mathrm{H})$.

${ }^{31}$ P NMR (162 MHz, $\left.\mathbf{C D C l}_{3}\right): \delta 21.87(\mathrm{~s})$.

\section{(E)-3-[(2-Furyl)methylidene]-1-[(R)-1-phenyl-2-propenyl]-5-chloro-2-indolinone (12)}

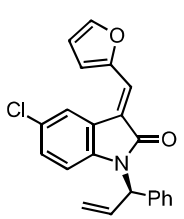

A flame-dried vial was charged with Wittig salt $\mathbf{S} 7(54.1 \mathrm{mg}, 0.128 \mathrm{mmol}, 1.20$ equiv) and another flamedried vial was charged with isatin 9da $(21.7 \mathrm{mg}, 0.106 \mathrm{mmol}, 1.00$ equiv). Both vials were sealed with septa and evacuated and backfilled with $\mathrm{N}_{2}$ three times, then freshly distilled THF $(0.7 \mathrm{~mL}$ to the vial containing $\mathbf{S} 7$, $0.3 \mathrm{~mL}$ to the vial containing $\mathbf{9 d a})$ was added. The suspension of $\mathbf{S 7}$ was cooled to $0{ }^{\circ} \mathrm{C}$, then ${ }^{n} \mathrm{BuLi}(2.48 \mathrm{M}$ in hexanes; $49 \mu \mathrm{L}, 0.12 \mathrm{mmol}, 1.15$ equiv) was added dropwise, causing the reaction to become dark reddish brown. After stirring for 10 minutes at $0{ }^{\circ} \mathrm{C}$, the cooling bath was removed and the reaction was stirred for an additional 5 minutes before it was added via syringe to the solution of $9 \mathbf{d a}$ at $0{ }^{\circ} \mathrm{C}$. After the addition was complete, the reaction was stirred for 20 minutes at $0{ }^{\circ} \mathrm{C}$, then quenched with acetone $(50 \mu \mathrm{L})$. The reaction was warmed to room temperature, then DMAP $(1.0 \mathrm{mg})$ was added in an attempt to equilibrate the $E / Z$ olefin isomers. By TLC, no change was observed after 2 hours, so the reaction was purified directly by preparatory TLC (6:1 heptane/EtOAc) to afford a top band, from which an 8:1 $\mathrm{Z} / E$ mixture was isolated ( $6.3 \mathrm{mg}, 16 \%$ ), and a bottom band, from which the pure $E$ isomer was isolated ( $23.5 \mathrm{mg}, 61 \%$ ). The stereochemistry of the newly formed olefin was assigned by analogy to the literature, ${ }^{16}$ and the stereochemistry of the $E$ isomer was further confirmed by nOe. ( $29.8 \mathrm{mg}, 78 \%$ combined)

Data for major E isomer

Physical appearance: Yellow solid

Melting point: $114-121^{\circ} \mathrm{C}$

TLC $\mathbf{R}_{\mathbf{f}}\left(3: 1\right.$ petroleum ether/ $\left.\mathbf{E t}_{2} \mathbf{O}, \mathbf{U V}\right): 0.26$

${ }^{1}$ H NMR (400 MHz, CDCl $): \delta 8.47(\mathrm{~d}, J=2.2 \mathrm{~Hz}, 1 \mathrm{H}), 7.83(\mathrm{~d}, J=1.8 \mathrm{~Hz}, 1 \mathrm{H}), 7.58(\mathrm{~s}, 1 \mathrm{H}), 7.33(\mathrm{~m}, 5 \mathrm{H}), 7.02(\mathrm{dd}, J=$ 8.4, $2.2 \mathrm{~Hz}, 1 \mathrm{H}), 6.98(\mathrm{~d}, J=3.5 \mathrm{~Hz}, 1 \mathrm{H}), 6.66(\mathrm{dd}, J=3.5,1.8 \mathrm{~Hz}, 1 \mathrm{H}), 6.43(\mathrm{~m}, 3 \mathrm{H}), 5.44(\mathrm{~m}, 1 \mathrm{H}), 5.35(\mathrm{~m}, 1 \mathrm{H})$.

${ }^{13}$ C NMR (101 MHz, CDCl $)$ ): $\delta 169.0(\mathrm{C}), 151.3(\mathrm{C}), 146.6(\mathrm{CH}), 140.3(\mathrm{C}), 137.5(\mathrm{C}), 132.9(\mathrm{CH}), 128.9(\mathrm{CH}), 128.5$

$(\mathrm{CH}), 127.8(\mathrm{CH}), 127.5(\mathrm{C}), 127.3(\mathrm{CH}), 125.1(\mathrm{CH}), 123.4(\mathrm{C}), 122.0(\mathrm{CH}), 121.3(\mathrm{CH}), 121.0(\mathrm{C}), 119.8\left(\mathrm{CH}_{2}\right), 113.5$ $(\mathrm{CH}), 112.0(\mathrm{CH}), 56.7(\mathrm{CH})$.

FT-IR (thin film): 3122, 3086, 3032, 2923, 2853, 1703, 1629, 1600, 1479, 1457, 1438, 1380, 1336, 1268, 1191, 1156, 1076, 1024, 935, 885, 810, 730, $700 \mathrm{~cm}^{-1}$.

HRMS: Calculated for $\mathrm{C}_{22} \mathrm{H}_{16} \mathrm{ClNO}_{2}[\mathrm{M}+\mathrm{H}]^{+}$362.0948, found 362.0943 .

$[\alpha]_{\mathbf{D}}{ }^{24}:+102.4^{\circ}\left(c=1.0, \mathrm{CHCl}_{3}\right)$ 
Data for minor $Z$ isomer

Physical appearance: Yellow gum

TLC $\mathbf{R}_{\mathrm{f}}\left(3: 1\right.$ petroleum ether/Et $\left.\mathbf{t}_{\mathbf{2}} \mathbf{O}, \mathrm{UV}\right): 0.57$

${ }^{1}$ H NMR (400 MHz, $\left.\mathbf{C D C l}_{3}\right): \delta 8.44(\mathrm{~d}, J=3.7 \mathrm{~Hz}, 1 \mathrm{H}), 7.65(\mathrm{~d}, J=1.7 \mathrm{~Hz}, 1 \mathrm{H}), 7.49$ (s, 1H), $7.47(\mathrm{~d}, J=2.1 \mathrm{~Hz}, 1 \mathrm{H})$, 7.33 (m, 5H), 6.98 (dd, $J=8.4,2.1 \mathrm{~Hz}, 1 \mathrm{H}), 6.65(\mathrm{~m}, 1 \mathrm{H}), 6.43(\mathrm{~m}, 3 \mathrm{H}), 5.44(\mathrm{~m}, 1 \mathrm{H}), 5.35(\mathrm{~m}, 1 \mathrm{H})$.

${ }^{13}$ C NMR (101 MHz, $\left.\mathbf{C D C l}_{3}\right): \delta 165.9(\mathrm{C}), 151.2(\mathrm{C}), 146.2(\mathrm{CH}), 138.5(\mathrm{C}), 137.5(\mathrm{C}), 133.0(\mathrm{CH}), 128.9(\mathrm{CH}), 127.9$ $(\mathrm{CH}), 127.4(\mathrm{CH}), 125.7(\mathrm{C}), 123.2(\mathrm{CH}), 121.0(\mathrm{CH}), 119.8\left(\mathrm{CH}_{2}\right), 119.1(\mathrm{CH}), 114.1(\mathrm{CH}), 112.2(\mathrm{CH}), 56.2(\mathrm{CH})$; Two quaternary carbons not observed due to insufficient material.

FT-IR (thin film): 3065, 3030, 2923, 2852, 1697, 1620, 1477, 1460, 1438, 1381, 1348, 1265, 1177, 1143, 1119, 1089, $1019,932,884,807,754,731,699,675 \mathrm{~cm}^{-1}$.

$[\alpha]_{\mathbf{D}}{ }^{24}:+165.4^{\circ}\left(c=1.0, \mathrm{CHCl}_{3}\right)$

1-[(S)-1-Methyl-2-propenyl $](Z)$-3-thiosemicarbazidoidene-2-indolinone (13)

S $-\mathrm{NH}_{2}$ A vial was charged with isatin 9af (22.1 mg, $0.110 \mathrm{mmol}, 1.00$ equiv), thiosemicarbazide (10.0 mg, 0.110 mmol, 1.00 equiv), and bulk $\mathrm{AcOH}(0.50 \mathrm{~mL})$. The vial was capped under ambient atmosphere, placed in a preheated $110{ }^{\circ} \mathrm{C}$ heating block, and stirred for 2 hours, during which time the color of the reaction changed from red-orange to yellow-brown. The reaction was cooled to room temperature and purified directly by preparatory TLC (2:1 petroleum ether/EtOAc) to afford the title compound as an orange crystalline solid that was $95 \%$ pure by ${ }^{1} \mathrm{H}$ NMR and could not be purified further. (29.8 $\left.\mathrm{mg}, 99 \%\right)$

Physical appearance: Orange crystals

Melting point: $176-179^{\circ} \mathrm{C}$

TLC $R_{\mathbf{f}}(4: 1$ petroleum ether/EtOAc, UV): 0.24

${ }^{1}$ H NMR (400 MHz, $\mathbf{C D C l}_{3}$ ): $\delta 12.90$ (bs, 1H), 7.59 (ddd, $\left.J=7.6,1.3,0.6 \mathrm{~Hz}, 1 \mathrm{H}\right), 7.57$ (bs, $\left.1 \mathrm{H}\right), 7.33$ (td, $J=7.8,1.3 \mathrm{~Hz}$, 1H), 7.09 (td, $J=7.6,0.9 \mathrm{~Hz}, 1 \mathrm{H}), 7.00$ (m, 1H), 6.79 (bs, 1H), 6.05 (ddd, $J=17.3,10.6,4.4 \mathrm{~Hz}, 1 \mathrm{H}), 5.30$ (m, $2 \mathrm{H}), 5.14$ $(\mathrm{m}, 1 \mathrm{H}), 1.58(\mathrm{~d}, J=7.1 \mathrm{~Hz}, 3 \mathrm{H})$.

${ }^{13}$ C NMR (101 MHz, CDCl $)$ ): $\delta 180.1(\mathrm{C}), 160.7(\mathrm{C}), 142.3(\mathrm{C}), 136.2(\mathrm{CH}), 132.2(\mathrm{C}), 131.3(\mathrm{CH}), 123.1(\mathrm{CH}), 121.0$ $(\mathrm{CH}), 119.8(\mathrm{C}), 117.3\left(\mathrm{CH}_{2}\right), 111.8(\mathrm{CH}), 48.7(\mathrm{CH}), 16.5\left(\mathrm{CH}_{3}\right)$.

FT-IR (thin film): 3422, 3259, 3157, 2982, 2930, 1687, 1611, 1468, 1361, 1314, 1210, 1143, 1099, 1063, 1014, 989, 915, $852,814,791,731 \mathrm{~cm}^{-1}$.

HRMS: Calculated for $\mathrm{C}_{13} \mathrm{H}_{14} \mathrm{~N}_{4} \mathrm{OS}[\mathrm{M}+\mathrm{H}]^{+} 275.0967$, found 275.0962 .

$[\alpha]_{\mathbf{D}}{ }^{24}:-13.6^{\circ}\left(c=1.0, \mathrm{CHCl}_{3}\right)$

\section{1-[(R)-1-(o-Tolyl)-2-propenyl]-3-[m-(trifluoromethyl)phenylimino]-2-indolinone (14)}

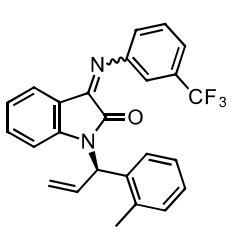

A vial was charged sequentially with isatin 9ab $(29.2 \mathrm{mg}, 0.105 \mathrm{mmol}, 1.00$ equiv), bulk EtOH $(0.5 \mathrm{~mL})$, bulk $\mathrm{AcOH}(1 \mu \mathrm{L}, 0.017 \mathrm{mmol}, 0.17$ equiv), and 3-(trifluoromethyl)aniline (13.5 $\mu \mathrm{L}, 0.108 \mathrm{mmol}, 1.03$ equiv). The vial was capped under ambient atmosphere, placed in a preheated $80{ }^{\circ} \mathrm{C}$ bath, and stirred overnight. The following day, progress had ceased by TLC, so powdered $3 \AA$ molecular sieves (30 mg) were added and the reaction was allowed to continue at $80^{\circ} \mathrm{C}$. After 7 hours, additional powdered $3 \AA$ molecular sieves were added and the reaction was stirred overnight at $80{ }^{\circ} \mathrm{C}$. The following day, progress had again ceased by TLC, so additional AcOH $(2 \mu \mathrm{L})$ and triethylorthoformate $(50 \mu \mathrm{L})$ were added, and the reaction was stirred overnight at $80{ }^{\circ} \mathrm{C}$. The following day, the reaction was still not complete, so it was purified directly by preparatory TLC (6:1 cyclohexane/EtOAc) to afford a top band, from which the desired product was isolated as a $4: 1$ mixture of inseparable imine isomers, ${ }^{17}$ and a bottom band, from which recovered 9ab was isolated (10.4 mg). (28.3 mg, 64\%; 99\% brsm) 
Physical appearance: Yellow oil

TLC R $R_{\text {f }}$ 6:1 cyclohexane/EtOAc, UV): 0.42

${ }^{1}$ H NMR (500 MHz, $\left.\mathbf{C D C l}_{3}\right): \delta 7.74^{*}(\mathrm{dd}, J=7.5,1.4 \mathrm{~Hz}, 0.25 \mathrm{H}), 7.55(\mathrm{~m}, \sim 4 \mathrm{H}), 7.28(\mathrm{~m}, \sim 7 \mathrm{H}), 7.14^{\dagger}(\mathrm{td}, J=7.9,1.3 \mathrm{~Hz}$, $1 \mathrm{H}), 7.08^{*}(\mathrm{td}, J=7.6,0.9 \mathrm{~Hz}, 0.25 \mathrm{H}), 6.71(\mathrm{~m}, 2.5 \mathrm{H}), 6.55^{\dagger}(\mathrm{dd}, J=7.7,1.3 \mathrm{~Hz}, 1 \mathrm{H}), 6.43^{\dagger}(\mathrm{m}, 2 \mathrm{H}), 6.35^{*}(\mathrm{ddd}, J=17.2$, $10.4,5.4 \mathrm{~Hz}, 0.25 \mathrm{H}), 6.16^{*}(\mathrm{~m}, 0.25 \mathrm{H}), 5.48(\mathrm{~m}, 1 \mathrm{H}), 5.44^{*}(\mathrm{ddd}, J=10.3,1.8,0.9 \mathrm{~Hz}, 0.25 \mathrm{H}), 5.33^{\dagger}(\mathrm{m}, 1 \mathrm{H}), 5.24^{*}(\mathrm{ddd}$, $J=17.2,1.9,0.9 \mathrm{~Hz}, 0.25 \mathrm{H}), 2.35^{\dagger}(\mathrm{s}, 3 \mathrm{H}), 2.25^{*}(\mathrm{~s}, 0.75 \mathrm{H}) ;^{\dagger}$ denotes peaks corresponding only the major isomer;

* denotes peaks corresponding only the the minor isomer.

${ }^{19}$ F NMR (376 MHz, $\mathbf{C D C l}_{3}$ ): $\delta-62.63$ (s, minor), -62.75 (s, major).

${ }^{13} \mathbf{C}$ NMR (126 MHz, $\left.\mathbf{C D C l}_{3}\right): \delta$ 162.6, 157.0, 154.8, 152.6, 150.8, 149.5, 147.4, 146.1, 137.6, 137.4, 135.0, 134.8, 134.4, $134.1,133.2,133.0,132.1$ (q, $J=32.6 \mathrm{~Hz}), 131.5,131.3,131.2(\mathrm{~m}), 130.2,129.1,128.6,128.5,128.4,128.1,126.3,126.3$, $126.1,124.2(\mathrm{~m}) 123.9$ (q, $J=272.5 \mathrm{~Hz}) 123.4,123.2,123.1,122.6,122.2(\mathrm{~m}), 122.0(\mathrm{q}, J=3.7 \mathrm{~Hz}), 121.9(\mathrm{~m}), 121.8$, $121.3,121.3,118.7,118.5,116.6(\mathrm{q}, J=3.9 \mathrm{~Hz}), 115.7,115.0(\mathrm{q}, J=3.8 \mathrm{~Hz}), 112.7,112.1,55.6,55.3,19.9,19.6$.

FT-IR (thin film): 3068, 3030, 2924, 2855, 1731, 1662, 1604, 1466, 1439, 1375, 1348, 1329, 1226, 1199, 1170, 1093, $1068,998,925,889,798,749,698,656 \mathrm{~cm}^{-1}$.

HRMS: Calculated for $\mathrm{C}_{25} \mathrm{H}_{19} \mathrm{~F}_{3} \mathrm{~N}_{2} \mathrm{O}[\mathrm{M}+\mathrm{H}]^{+} 421.1528$, found 421.1523 .

$[\boldsymbol{\alpha}]_{\mathbf{D}}{ }^{24}:+72.1^{\circ}\left(c=1.0, \mathrm{CHCl}_{3}\right)$

\section{2-[(R)-1-Phenyl-2-propenylamino]-5-chlorobenzoic acid (15)}

The title compound was prepared using literature conditions. ${ }^{6} \mathrm{~A}$ vial was charged with isatin 9da (41.4 mg, $0.139 \mathrm{mmol}, 1.00$ equiv) and bulk THF $(0.5 \mathrm{~mL})$ and the resulting orange solution was cooled to $0{ }^{\circ} \mathrm{C}$ under ambient atmosphere. An aqueous solution of $2.0 \mathrm{M}$ sodium hydroperoxide [0.32 mL, $0.63 \mathrm{mmol}, 4.5$ equiv; prepared by diluting $\mathrm{NaOH}(800 \mathrm{mg}, 20.0 \mathrm{mmol})$ and $30 \% \mathrm{H}_{2} \mathrm{O}_{2}(2.04 \mathrm{~mL}, 20.0 \mathrm{mmol})$ to $10.0 \mathrm{~mL}$ in a

volumetric flask] was added dropwise, during which time the reaction changed color from orange to light yellow. The reaction was stirred for $25 \mathrm{~min}$ at $0{ }^{\circ} \mathrm{C}$, then saturated $\mathrm{Na}_{2} \mathrm{SO}_{3}(0.3 \mathrm{~mL})$ was added. After stirring for approximately 10 minutes, concentrated $\mathrm{HCl}(53 \mu \mathrm{L}, 4.6$ equiv) was added dropwise. The layers were separated, then the aqueous phase was extracted with EtOAc $(4 \times 0.5 \mathrm{~mL})$. The combined organic layers were passed through a plug of $\mathrm{MgSO}_{4}$ and concentrated in vacuo to afford a light tan foam that was pure by ${ }^{1} \mathrm{H}$ NMR and was fully characterized without purification. (40.4 mg, quant.)

Physical appearance: Light tan foam

Melting point: $54-60{ }^{\circ} \mathrm{C}$

TLC $R_{\mathbf{f}}$ (2:1 petroleum ether/acetone w/ 1\% v/v AcOH, UV): 0.33

${ }^{1}$ H NMR (300 MHz, $\left.\mathbf{C D C l}_{3}, \mathbf{5 0}^{\circ} \mathbf{C}\right): \delta 8.65$ (bs, 2H), $7.90(\mathrm{~d}, J=2.6 \mathrm{~Hz}, 1 \mathrm{H}), 7.23(\mathrm{~m}, 6 \mathrm{H}), 6.49(\mathrm{~d}, J=9.1 \mathrm{~Hz}, 1 \mathrm{H}), 6.00$ (ddd, $J=16.5,10.1,5.7 \mathrm{~Hz}, 1 \mathrm{H}), 5.09(\mathrm{~m}, 3 \mathrm{H})$; signals were broad at rt, presumably due to aggregation.

${ }^{13} \mathbf{C}$ NMR (75 MHz, $\left.\mathbf{C D C l}_{3}, 50{ }^{\circ} \mathbf{C}\right): \delta 173.3$ (br, C), $149.2(\mathrm{C}), 141.2(\mathrm{C}), 138.6(\mathrm{CH}), 135.2(\mathrm{CH}), 131.9(\mathrm{CH}), 129.0$ $(\mathrm{CH}), 127.7(\mathrm{CH}), 127.0(\mathrm{CH}), 120.2(\mathrm{C}), 116.3\left(\mathrm{CH}_{2}\right), 114.6(\mathrm{CH}), 111.4(\mathrm{br}, \mathrm{C}), 60.2(\mathrm{CH})$.

FT-IR (thin film): 3371, 3026, 2917, 2623, 2543, 1667, 1574, 1504, 1443, 1416, 1274, 1316, 1228, 1161, 1120, 1077, $1028,990,908,882,811,755,733,701,658 \mathrm{~cm}^{-1}$.

HRMS: Calculated for $\mathrm{C}_{16} \mathrm{H}_{14} \mathrm{ClNO}_{2}[\mathrm{M}-\mathrm{H}]^{-} 286.0635$, found 286.0637.

$[\alpha]_{\mathbf{D}}{ }^{24}:-128.4^{\circ}\left(c=1.0, \mathrm{CHCl}_{3}\right)$ 
17-[(R)-1-Phenyl-2-propenyl]-13-chloro-5,6-dimethyl-2,9,17-triazatetracyclo $\left[8.7 .0 .0^{3,8} .0^{11,16}\right]$ heptadeca$1,3,5,7,9,11(16), 12,14-$ octaene (16)

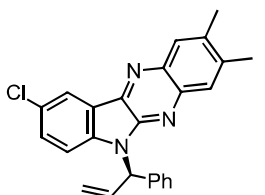

A vial was charged sequentially with isatin 9da $(22.3 \mathrm{mg}, 0.0749 \mathrm{mmol}, 1.00$ equiv), 4,5dimethylbenzene-1,2-diamine (10.2 mg, $0.0749 \mathrm{mmol}, 1.00$ equiv), and bulk $\mathrm{AcOH}(0.5 \mathrm{~mL})$. The vial was capped under ambient atmosphere, stirred for 5 minutes at room temperature, then placed in a preheated $110{ }^{\circ} \mathrm{C}$ heating block and stirred for 1 hour. The reaction was cooled to room temperature, concentrated in vacuo, and purified by flash chromatography (1.5 g silica, 22:1 petroleum ether/acetone) to afford a yellow solid that was further purified by preparatory TLC (20:1 heptane/acetone) to remove a trace - but highly UV-active impurity. (18.6 mg, 62\%)

Physical appearance: Light yellow solid

Melting point: $178-181^{\circ} \mathrm{C}$

TLC $\mathbf{R}_{\mathbf{f}}$ (19:1 petroleum ether/acetone, $\left.\mathbf{U V}\right): 0.36$

${ }^{1} \mathbf{H}$ NMR (400 MHz, CDCl $): \delta 8.42(\mathrm{~d}, J=2.2 \mathrm{~Hz}, 1 \mathrm{H}), 8.05(\mathrm{~s}, 1 \mathrm{H}), 7.90(\mathrm{~s}, 1 \mathrm{H}), 7.35(\mathrm{~m}, 6 \mathrm{H}), 7.15(\mathrm{~d}, J=6.4 \mathrm{~Hz}, 1 \mathrm{H})$, 7.07 (d, $J=8.7 \mathrm{~Hz}, 1 \mathrm{H}), 6.65$ (ddd, $J=16.9,10.4,6.4 \mathrm{~Hz}, 1 \mathrm{H}), 5.45$ (dd, $J=10.3,1.3 \mathrm{~Hz}, 1 \mathrm{H}), 5.33(\mathrm{dd}, J=17.1,1.4 \mathrm{~Hz}$, $1 \mathrm{H}), 2.54(\mathrm{~s}, 6 \mathrm{H})$.

${ }^{13}$ C NMR (101 MHz, CDCl $): \delta 145.9$ (C), 141.1 (C), 140.0 (C), 139.8 (C), 138.9 (C), 138.1 (C), 138.0 (C), 136.8 (C), $133.6(\mathrm{CH}), 129.9(\mathrm{CH}), 128.9(\mathrm{CH}), 128.6(\mathrm{CH}), 128.0(\mathrm{CH}), 127.4(\mathrm{CH}), 127.3(\mathrm{CH}), 126.6(\mathrm{C}), 122.2(\mathrm{CH}), 121.7(\mathrm{C})$, $119.7\left(\mathrm{CH}_{2}\right), 113.5(\mathrm{CH}), 58.0(\mathrm{CH}), 20.6\left(\mathrm{CH}_{3}\right), 20.3\left(\mathrm{CH}_{3}\right)$.

FT-IR (thin film): 3064, 3033, 2967, 2919, 2851, 1580, 1493, 1455, 1385, 1344, 1273, 1211, 1171, 1135, 1079, 1049, 1001, 932, 872, 805, $768 \mathrm{~cm}^{-1}$.

HRMS: Calculated for $\mathrm{C}_{25} \mathrm{H}_{20} \mathrm{ClN}_{3}[\mathrm{M}+\mathrm{H}]^{+} 398.1424$, found 398.1419 .

$[\alpha]_{\mathrm{D}}{ }^{24}:+110.7^{\circ}\left(c=1.0, \mathrm{CHCl}_{3}\right)$

\section{8-[(R)-1-Phenyl-2-propenyl]-4,5-dichloro-13,14-dimethyl-8,10,17-triazatetracyclo $\left[8.7 .0 .0^{2,7} \cdot 0^{11,16}\right]$ heptadeca- 1(17),2(7),3,5,11,13,15-heptaen-9-one (17)}

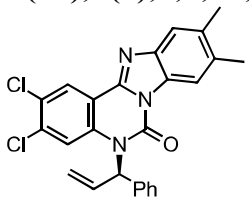

The title compound was prepared using a method developed by Ma. ${ }^{18} \mathrm{~A}$ vial was sequentially charged with isatin 9 ja $(23.1 \mathrm{mg}, 0.0695 \mathrm{mmol}, 1.00$ equiv), 4,5-dimethylbenzene-1,2-diamine $(9.5 \mathrm{mg}, 0.70$ mmol, 1.0 equiv), and bulk DMSO $(0.7 \mathrm{~mL})$. The resulting solution was stirred at room temperature for roughly one minute before TBHP ( $5-6 \mathrm{M}$ in decane; $70 \mu \mathrm{L}, 0.35 \mathrm{mmol}, 5.0$ equiv) was added. The resulting mixture was then placed in a preheated $90{ }^{\circ} \mathrm{C}$ bath and stirred open to the atmosphere. After 30 minutes, some product formation was observed by TLC, and catalytic $\mathrm{AcOH}(1.0 \mu \mathrm{L})$ was added. The reaction was stirred at $90{ }^{\circ} \mathrm{C}$ for an additional 2.5 hours, during which time the color changed from dark purple to light brown and a tan-colored solid precipitated. The reaction was cooled to room temperature, diluted with water $(6.5 \mathrm{~mL})$ and saturated $\mathrm{Na}_{2} \mathrm{SO}_{3}(0.5 \mathrm{~mL})$, and extracted with EtOAc $(3 \times 3 \mathrm{~mL})$. The combined organic extracts were concentrated in vacuo to a brown solid that was poorly soluble in most organic solvents, but very soluble in $\mathrm{CHCl}_{3}$. This solid was purified by preparatory TLC (9:1 petroleum ether/EtOAc) to afford the title compound as a cream-colored solid. (16.1 mg, 52\%)

Physical appearance: Cream-colored solid

Melting point: $225-228^{\circ} \mathrm{C}$

TLC $\mathbf{R}_{\mathbf{f}}$ (9:1 petroleum ether/EtOAc, UV): 0.31

${ }^{1}$ H NMR (400 MHz, CDCl $)$ ): $\delta 8.57(\mathrm{~s}, 1 \mathrm{H}), 8.26(\mathrm{~s}, 1 \mathrm{H}), 7.65(\mathrm{~s}, 1 \mathrm{H}), 7.36(\mathrm{~m}, 7 \mathrm{H}), 6.64(\mathrm{ddd}, J=17.0,10.4,6.6 \mathrm{~Hz}, 1 \mathrm{H})$, $5.59(\mathrm{~d}, J=10.4 \mathrm{~Hz}, 1 \mathrm{H}), 5.50(\mathrm{~d}, J=17.0 \mathrm{~Hz}, 1 \mathrm{H}), 2.46(\mathrm{~s}, 3 \mathrm{H}), 2.45(\mathrm{~s}, 3 \mathrm{H})$.

${ }^{13}$ C NMR (101 MHz, CDCl $)$ ): $\delta 147.5$ (C), 144.3 (C), 142.5 (C), 137.2 (C), 135.4 (C), 135.0 (C), 134.8 (C), 134.6 (C), $132.0(\mathrm{CH}), 129.9(\mathrm{C}), 129.4(\mathrm{CH}), 128.2(\mathrm{CH}), 128.2(\mathrm{C}), 126.5(\mathrm{CH}), 126.3(\mathrm{CH}), 121.9\left(\mathrm{CH}_{2}\right), 119.9(\mathrm{CH}), 119.9(\mathrm{CH})$, 
115.7 (CH), $114.3(\mathrm{C}), 59.6(\mathrm{CH}), 20.8\left(\mathrm{CH}_{3}\right)$; one less peak than expected due to merging of $\mathrm{CH}_{3}$ signals.

FT-IR (thin film): 3066, 3034, 2923, 2855, 1701, 1628, 1610, 1580, 1548, 1446, 1371, 1305, 1281, 1255, 1221, 1134, 1022, 1000, 941, 910, 864, 826, 777, 731, $699 \mathrm{~cm}^{-1}$.

HRMS: Calculated for $\mathrm{C}_{25} \mathrm{H}_{19} \mathrm{Cl}_{2} \mathrm{~N}_{3} \mathrm{O}[\mathrm{M}+\mathrm{H}]^{+} 448.0983$, found 448.0977 .

$[\alpha]_{\mathbf{D}}^{24}:+126.9^{\circ}\left(c=1.0, \mathrm{CHCl}_{3}\right)$

\section{1-[(R)-1-(o-Tolyl)-2-propenyl]-3-hydroxy-4-phenyl-1 H-quinolin-2-one (19)}

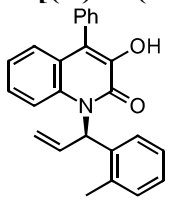

The title compound was prepared using a method developed by Babu. ${ }^{19} \mathrm{~A}$ vial was charged sequentially with benzaldehyde (10.6 mg, $0.100 \mathrm{mmol}, 1.00$ equiv), bulk EtOH $(0.5 \mathrm{~mL})$, and tosyl hydrazone (18.6 mg, 0.100 mmol, 1.00 equiv). The resulting solution was stirred at room temperature for 30 minutes, at which point the

benzaldehyde was consumed by TLC, then isatin $9 \mathbf{a b}\left(27.7 \mathrm{mg}, 0.100 \mathrm{mmol}, 1.00\right.$ equiv) and $\mathrm{K}_{2} \mathrm{CO}_{3}(27.6$ $\mathrm{mg}, 0.100 \mathrm{mmol}, 2.00$ equiv) were added sequentially. The vial was capped, placed in a preheated $80{ }^{\circ} \mathrm{C}$ heating block, and stirred for 3.5 hours under ambient atmosphere. The reaction was cooled to room temperature, quenched with $\mathrm{AcOH}(20 \mu \mathrm{L}$ $\mathrm{mL}$ ) and purified directly by preparatory TLC to afford a top band, from which the title compound was isolated (22.2 $\mathrm{mg}$, $61 \%)$, and a bottom band, from which a regioisomeric ring expansion product $\mathbf{2 0}$ was isolated (12.1 $\mathrm{mg}, 33 \%)$. The structure of $\mathbf{2 0}$ was assigned based on a comparison of the NMR data to those reported for similar compounds, ${ }^{20}$ and was further supported by nOe data.

\section{Data for major product 19}

Physical appearance: Light orange solid

Melting point: $138-145^{\circ} \mathrm{C}$

${ }^{1}$ H NMR (400 MHz, $\left.\mathbf{C D C l}_{3}\right): \delta 7.59(\mathrm{~d}, J=7.6 \mathrm{~Hz}, 1 \mathrm{H}), 7.52(\mathrm{~m}, 2 \mathrm{H}), 7.46(\mathrm{td}, J=8.1,7.4,3.2 \mathrm{~Hz}, 4 \mathrm{H}), 7.34(\mathrm{dd}, J=8.0$, $1.6 \mathrm{~Hz}, 1 \mathrm{H}), 7.20(\mathrm{~m}, 6 \mathrm{H}), 7.06(\mathrm{~m}, 1 \mathrm{H}), 6.62(\mathrm{ddd}, J=17.2,10.4,5.7 \mathrm{~Hz}, 1 \mathrm{H}), 5.48(\mathrm{dt}, J=10.5,1.4 \mathrm{~Hz}, 1 \mathrm{H}), 5.28(\mathrm{dt}, J=$ $17.2,1.3 \mathrm{~Hz}, 1 \mathrm{H}), 2.07(\mathrm{~s}, 3 \mathrm{H})$.

${ }^{13}$ C NMR (101 MHz, CDCl $): \delta 159.6(\mathrm{C}), 140.5(\mathrm{C}), 137.0(\mathrm{C}), 136.6(\mathrm{C}), 133.8(\mathrm{CH}), 133.5(\mathrm{C}), 133.1(\mathrm{C}), 131.5(\mathrm{CH})$, $130.1(\mathrm{CH}), 128.7(\mathrm{CH}), 128.5(\mathrm{CH}), 128.4(\mathrm{CH}), 128.1(\mathrm{CH}), 126.4(\mathrm{CH}), 126.4(\mathrm{CH}), 126.2(\mathrm{CH}), 123.9(\mathrm{C}), 123.0(\mathrm{CH})$, $122.7(\mathrm{C}), 118.7\left(\mathrm{CH}_{2}\right), 117.2(\mathrm{CH}), 59.8(\mathrm{CH}), 19.7\left(\mathrm{CH}_{3}\right)$.

FT-IR (thin film): 3307, 3062, 3029, 2924, 1854, 1739, 1618, 1599, 1493, 1461, 1399, 1325, 1285, 1223, 1163, 1133 , $1097,1055,1032,985,910,958,734,701,670 \mathrm{~cm}^{-1}$.

HRMS: Calculated for $\mathrm{C}_{25} \mathrm{H}_{21} \mathrm{NO}_{2}[\mathrm{M}+\mathrm{H}]^{+} 368.1651$, found 368.1646.

$[\alpha]_{\mathbf{D}}{ }^{24}:+161.2^{\circ}\left(c=1.0, \mathrm{CHCl}_{3}\right)$

\section{Data for minor product 20}

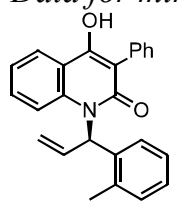

Physical appearance: Clear colorless gum

${ }^{1}$ H NMR (500 MHz, $\left.\mathbf{C D C l}_{3}\right): \delta 8.01(\mathrm{dd}, J=8.0,1.6 \mathrm{~Hz}, 1 \mathrm{H}), 7.54(\mathrm{~m}, 3 \mathrm{H}), 7.49(\mathrm{~m}, 2 \mathrm{H}), 7.43(\mathrm{~m}, 2 \mathrm{H}), 7.36$ $(\mathrm{d}, J=4.3 \mathrm{~Hz}, 1 \mathrm{H}), 7.29(\mathrm{~m}, 1 \mathrm{H}), 7.22(\mathrm{~m}, 1 \mathrm{H}), 7.16(\mathrm{~m}, 2 \mathrm{H}), 7.08(\mathrm{~d}, J=7.4 \mathrm{~Hz}, 1 \mathrm{H}), 6.55(\mathrm{ddd}, J=17.2$, $10.4,5.5 \mathrm{~Hz}, 1 \mathrm{H}), 6.11(\mathrm{~s}, 1 \mathrm{H}), 5.41(\mathrm{ddd}, J=10.4,1.8,1.1 \mathrm{~Hz}, 1 \mathrm{H}), 5.22(\mathrm{ddd}, J=17.2,1.9,1.1 \mathrm{~Hz}, 1 \mathrm{H})$, $2.11(\mathrm{~s}, 3 \mathrm{H})$.

${ }^{13}$ C NMR (75 MHz, $\left.\mathbf{C D C l}_{3}\right): \delta$ 162.7, 155.9, 138.4, 137.6, 137.3, 134.4, 131.5, 131.4, 130.8, 130.1, 129.7, 128.9, 128.4, $127.67,126.0,124.0,121.8,118.2,117.3,115.9,58.0,19.7$; one less signal then expected.

FT-IR (thin film): 3500, 3235, 3197, 3061, 3028, 2926, 2855, 1726, 1628, 1595, 1568, 1532, 1500, 1458, 1411, 1385, $1326,1298,1269,12141141,1061,1029,998,911,856,790,755,731,698 \mathrm{~cm}^{-1}$. 


\section{Crystallographic Data}
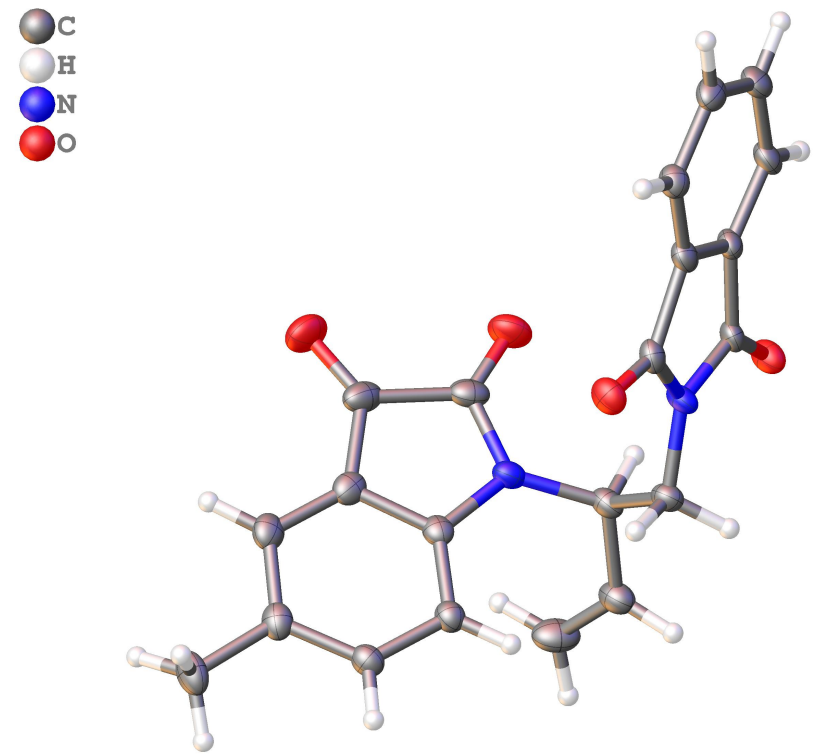

Table S1. Crystal data and structure refinement for $\mathbf{9 m j}$.

Identification code

Empirical formula

Formula weight

Temperature/K

Crystal system

Space group

$\mathrm{a} / \AA$

$\mathrm{b} / \AA$

$\mathrm{c} / \AA$

$\alpha /^{\circ}$

$\beta /{ }^{\circ}$

$\gamma /{ }^{\circ}$

Volume $/ \AA^{3}$

Z

$\rho_{\text {calc }} \mathrm{g} / \mathrm{cm}^{3}$

$\mu / \mathrm{mm}^{-1}$

$\mathrm{F}(000)$

Crystal size $/ \mathrm{mm}^{3}$

Radiation

$2 \Theta$ range for data collection/ ${ }^{\circ}$

Index ranges

Reflections collected

Independent reflections

Data/restraints/parameters

Goodness-of-fit on $\mathrm{F}^{2}$

Final $\mathrm{R}$ indexes $[\mathrm{I}>=2 \sigma(\mathrm{I})]$

Final $\mathrm{R}$ indexes [all data]

Largest diff. peak/hole / e $\AA^{-3}$

Flack parameter 9mj

$\mathrm{C}_{126} \mathrm{H}_{96} \mathrm{~N}_{12} \mathrm{O}_{24}$

2162.14

100

monoclinic

B $2 \mathrm{yb}$

15.5993(8)

49.062(2)

15.6099(8)

90

119.896(2)

90

10357.0(9)

4

1.387

0.802

4512.0

$0.68 \times 0.073 \times 0.073$

$\mathrm{CuK} \alpha(\lambda=1.54178)$

3.602 to 160.338

$-18 \leq \mathrm{h} \leq 19,-62 \leq \mathrm{k} \leq 62,-19 \leq 1 \leq 19$

349707

$43677\left[\mathrm{R}_{\mathrm{int}}=0.0579, \mathrm{R}_{\text {sigma }}=0.0357\right]$

$43677 / 1 / 1468$

1.080

$\mathrm{R}_{1}=0.0440, \mathrm{wR}_{2}=0.1138$

$\mathrm{R}_{1}=0.0497, \mathrm{wR}_{2}=0.1211$

$0.20 /-0.24$

$0.003(47)$ 
Table S2. Fractional Atomic Coordinates $\left(\times 10^{4}\right)$ and Equivalent Isotropic Displacement Parameters $\left(\AA^{2} \times 10^{3}\right)$ for $9 \mathrm{mj} . \mathrm{U}_{\mathrm{eq}}$ is defined as $1 / 3$ of of the trace of the orthogonalised $\mathrm{U}_{\mathrm{IJ}}$ tensor.

\begin{tabular}{|c|c|c|c|c|}
\hline Atom & $x$ & & & \\
\hline O1_1 & 7961(3) & $6996.6(8)$ & $3393(3)$ & $32.9(9)$ \\
\hline $\mathrm{O} 2 \_1$ & 6413(3) & $7569.2(8)$ & $737(3)$ & $29.7(8)$ \\
\hline O3_1 & 4598(3) & $7059.4(9)$ & $938(3)$ & $36.2(9)$ \\
\hline O4_1 & 4072(3) & $6588.2(10)$ & 1751(3) & $40.0(10)$ \\
\hline N1_1 & 7189(3) & $7233.8(8)$ & $1914(3)$ & $22.4(9)$ \\
\hline $\mathrm{N} 2-1$ & 5795(4) & 6757.1(9) & $1035(3)$ & $26.0(9)$ \\
\hline $\mathrm{Cl}{ }_{-}^{-} 1$ & 7554(4) & $7201.1(11)$ & $2925(4)$ & $24.7(11)$ \\
\hline $\mathrm{C} 2 \_1$ & 7332(4) & $7461.4(11)$ & $3261(4)$ & $22.7(10)$ \\
\hline C3_- 1 & $7539(5)$ & $7544.2(12)$ & $4194(4)$ & $29.9(12)$ \\
\hline $\mathrm{C} 4 \_1$ & $7206(5)$ & $7799.9(12)$ & $4264(5)$ & $33.8(13)$ \\
\hline $\mathrm{C} 5 \_1$ & 6704(5) & $7970.0(12)$ & $3455(5)$ & $32.3(13)$ \\
\hline C6_1 & $6509(5)$ & $7888.0(12)$ & $2519(5)$ & $29.2(11)$ \\
\hline C7_-1 & 6832(4) & $7632.3(11)$ & $2448(4)$ & $22.9(11)$ \\
\hline C8_1 & 6752(4) & $7489.9(11)$ & $1577(4)$ & $24.5(10)$ \\
\hline C9_1 & $7227(4)$ & $7032.7(10)$ & $1254(4)$ & $26.0(10)$ \\
\hline C10_1 & 6193(4) & $6924.8(10)$ & $538(4)$ & 26.1(10) \\
\hline C11_1 & $6105(4)$ & $6779.0(10)$ & $-345(3)$ & $31.2(11)$ \\
\hline C12_1 & 6759(4) & $6770.7(10)$ & $-643(4)$ & $28.7(11)$ \\
\hline C13_1 & 6054(4) & $6485.4(11)$ & $1340(4)$ & $25.5(11)$ \\
\hline C14_1 & $6815(4)$ & $6330.3(10)$ & $1381(4)$ & 27.1(10) \\
\hline C15_1 & 6898(4) & $6058.1(10)$ & $1679(4)$ & 29.1(10) \\
\hline C16_1 & $6259(4)$ & $5941.0(9)$ & $1966(3)$ & 29.1(10) \\
\hline $\mathrm{C} 17_{-}^{-} 1$ & 6390(4) & $5646.2(10)$ & $2286(4)$ & $36.4(11)$ \\
\hline C18_1 & $5523(4)$ & $6105.1(11)$ & $1959(3)$ & $28.5(10)$ \\
\hline C19_1 & $5427(4)$ & $6372.3(12)$ & $1651(4)$ & $28.2(11)$ \\
\hline C20_1 & 4735(4) & $6586.2(13)$ & $1557(4)$ & $30.5(12)$ \\
\hline $\mathrm{C} 21_{-}^{-} 1$ & 4994(5) & $6839.9(12)$ & $1126(4)$ & $29.0(11)$ \\
\hline O1_2 & 7783(3) & $6992.0(8)$ & $8077(3)$ & $36.7(9)$ \\
\hline $\mathrm{O} 2 \_2$ & 6199(3) & $7624.2(7)$ & $5627(3)$ & $31.4(8)$ \\
\hline O3_2 & 4390(3) & $7044.6(8)$ & $5831(3)$ & $30.3(8)$ \\
\hline O4_2 & 4284(3) & $6575.6(9)$ & $6950(3)$ & $34.2(9)$ \\
\hline N1_2 & 6996(3) & $7265.7(8)$ & $6686(3)$ & $24.4(9)$ \\
\hline N2_2 & $5651(3)$ & $6785.2(9)$ & $5865(3)$ & $22.2(8)$ \\
\hline C1_2 & 7393(4) & $7206.0(11)$ & $7693(4)$ & 27.3(11) \\
\hline $\mathrm{C} 2 \_2$ & $7218(4)$ & $7455.5(11)$ & $8126(4)$ & $26.3(11)$ \\
\hline C3_2 & $7464(5)$ & $7512.7(14)$ & $9088(5)$ & $36.6(14)$ \\
\hline C4_2 & $7158(5)$ & $7763.8(14)$ & $9248(5)$ & $40.3(16)$ \\
\hline C5_2 & $6647(5)$ & 7949.3(13) & $8502(5)$ & $36.4(14)$ \\
\hline C6_2 & 6413(5) & 7893.3(12) & $7537(5)$ & $30.1(12)$ \\
\hline C7_2 & 6712(4) & $7643.9(11)$ & $7375(4)$ & 24.1(11) \\
\hline C8_2 & 6574(4) & $7526.0(10)$ & $6446(4)$ & $23.4(10)$ \\
\hline C9_2 & 6974(4) & $7082.5(9)$ & $5950(4)$ & $24.9(10)$ \\
\hline C10_2 & 5926(4) & $6966.4(9)$ & $5299(3)$ & $23.3(10)$ \\
\hline C11_2 & $5756(4)$ & $6827.3(8)$ & $4372(3)$ & $24.0(10)$ \\
\hline C12_2 & $6425(4)$ & $6796.5(10)$ & $4095(4)$ & $31.3(12)$ \\
\hline $\mathrm{C} 13{ }_{-}^{-} 2$ & $6085(4)$ & $6528.9(10)$ & $6249(4)$ & 19.9(9) \\
\hline C14_2 & 6855(4) & 6399.8(9) & $6209(4)$ & 21.3(9) \\
\hline
\end{tabular}




\begin{tabular}{|c|c|c|c|c|}
\hline C15_2 & $7142(3)$ & $6142.5(9)$ & $6642(3)$ & $22.2(9)$ \\
\hline C16_2 & 6691(3) & $6013.3(8)$ & $7120(3)$ & 22.9(9) \\
\hline C17_2 & 7039(3) & $5737.8(8)$ & $7588(3)$ & $28.5(9)$ \\
\hline C18_2 & 5914(3) & $6149.2(9)$ & $7142(3)$ & 24.2(9) \\
\hline C19_2 & $5629(4)$ & $6402.7(10)$ & $6721(4)$ & $22.6(10)$ \\
\hline $\mathrm{C} 20 \_2$ & 4863(4) & $6591.5(11)$ & $6649(4)$ & $24.5(10)$ \\
\hline $\mathrm{C} 21 \_2$ & 4899(4) & $6842.2(11)$ & $6056(4)$ & $23.8(10)$ \\
\hline O1_ 5 & 4309(3) & $3630.8(7)$ & 2241(3) & $28.0(8)$ \\
\hline $\mathrm{O} 2 \_5$ & $6809(2)$ & $4252.0(7)$ & $3107(3)$ & $28.5(7)$ \\
\hline O3_5 & $6573(2)$ & $3712.3(6)$ & $1143(2)$ & $31.4(7)$ \\
\hline O4_5 & $5605(2)$ & $3233.9(7)$ & $-129(2)$ & $31.7(7)$ \\
\hline N1_5 & $5729(3)$ & $3897.1(8)$ & $2839(3)$ & $21.4(8)$ \\
\hline $\mathrm{N} 2-5$ & 6603(3) & $3427.3(7)$ & $2333(3)$ & 21.2(7) \\
\hline C1_5 & $4712(4)$ & $3841.7(9)$ & $2245(4)$ & $21.5(10)$ \\
\hline $\mathrm{C} 2-5$ & $4272(4)$ & 4093.2(9) & $1638(3)$ & 20.4(9) \\
\hline C3_5 & $3303(3)$ & 4155.1(9) & $936(3)$ & 24.9(9) \\
\hline C4_5 & $3130(4)$ & $4406.5(11)$ & $453(3)$ & $29.4(10)$ \\
\hline C5_5 & $3908(4)$ & $4588.0(10)$ & $686(4)$ & 28.1(11) \\
\hline C6_5 & 4873(4) & $4525.1(9)$ & 1397(3) & $26.7(9)$ \\
\hline C7_5 & $5048(4)$ & 4276.1(9) & $1873(4)$ & $23.5(10)$ \\
\hline C8_5 & 5973(3) & 4155.7(9) & $2660(3)$ & 20.6(9) \\
\hline C9_5 & $6465(3)$ & $3713.9(9)$ & $3558(3)$ & 24.6(9) \\
\hline C10_5 & $7147(3)$ & $3606.8(8)$ & $3192(3)$ & $22.3(8)$ \\
\hline C11_5 & $8083(3)$ & $3470.5(8)$ & $3970(3)$ & 24.1(8) \\
\hline C12_5 & 8371(3) & $3447.5(9)$ & $4918(3)$ & 25.4(9) \\
\hline C13_5 & $6278(3)$ & $3157.4(9)$ & $2348(3)$ & 20.3(9) \\
\hline C14_5 & 6322(4) & $3016.7(10)$ & $3125(4)$ & $23.1(10)$ \\
\hline C15_5 & $5967(4)$ & $2747.0(10)$ & $2948(4)$ & $24.6(10)$ \\
\hline $\mathrm{C} 16 \_5$ & $5572(4)$ & $2622.8(10)$ & $2035(4)$ & $25.7(10)$ \\
\hline C17_5 & $5174(4)$ & $2334.2(10)$ & $1868(4)$ & $31.6(11)$ \\
\hline $\mathrm{C} 18 \_5$ & $5516(3)$ & $2772.3(9)$ & $1246(4)$ & $23.9(10)$ \\
\hline C19_5 & $5864(3)$ & $3039.0(10)$ & $1409(3)$ & 23.1(9) \\
\hline C20_5 & $5902(3)$ & $3241.5(9)$ & $754(3)$ & $24.3(8)$ \\
\hline C21_5 & $6403(3)$ & $3497.3(10)$ & $1402(3)$ & 25.7(9) \\
\hline O1_ 6 & $4152(3)$ & $3705.6(7)$ & $7024(3)$ & $27.3(7)$ \\
\hline O2_6 & $6828(2)$ & $4280.9(6)$ & $8522(2)$ & $23.4(6)$ \\
\hline O3_6 & $6965(2)$ & $3812.1(6)$ & $6769(3)$ & $32.8(7)$ \\
\hline O4_6 & $6237(3)$ & $3355.3(7)$ & $5338(2)$ & $35.7(7)$ \\
\hline N1_6 & $5617(3)$ & $3945.6(7)$ & $7950(3)$ & $19.4(8)$ \\
\hline N2_6 & 6771(3) & $3491.4(7)$ & $7746(3)$ & $21.6(7)$ \\
\hline C1_6 & $4640(4)$ & $3910.7(10)$ & $7180(4)$ & $21.2(10)$ \\
\hline $\mathrm{C} 2 \_6$ & $4362(4)$ & $4176.2(9)$ & $6629(3)$ & 19.1(9) \\
\hline C3_6 & $3478(4)$ & $4262.8(9)$ & $5825(3)$ & $24.8(9)$ \\
\hline C4_6 & $3442(4)$ & $4527.4(9)$ & $5476(4)$ & $25.5(10)$ \\
\hline C5_6 & $4277(3)$ & 4694.1(8) & $5929(3)$ & 23.8(9) \\
\hline C6_6 & $5168(3)$ & $4606.6(8)$ & $6730(3)$ & 22.9(9) \\
\hline C7_6 & 5190(4) & $4346.8(9)$ & $7073(3)$ & 19.7(9) \\
\hline C8_6 & 5990(3) & $4201.9(9)$ & $7923(3)$ & 19.3(9) \\
\hline C9_6 & 6197(3) & $3746.9(8)$ & $8716(3)$ & 21.1(9) \\
\hline C10_6 & $7095(3)$ & $3653.4(8)$ & $8638(3)$ & $19.8(8)$ \\
\hline
\end{tabular}




\begin{tabular}{|c|c|c|c|c|}
\hline C11_6 & $7879(3)$ & $3513.8(8)$ & $9567(3)$ & $24.2(8)$ \\
\hline C12_6 & $8518(3)$ & $3333.9(9)$ & $9606(4)$ & $30.5(9)$ \\
\hline C13_6 & $6388(3)$ & $3223.2(9)$ & $7600(3)$ & $19.5(9)$ \\
\hline C14_6 & $6243(4)$ & $3065.5(9)$ & $8239(4)$ & $21.5(10)$ \\
\hline C15_6 & $5879(4)$ & $2801.3(10)$ & $7946(4)$ & $24.0(10)$ \\
\hline C16_6 & $5647(4)$ & $2695.8(10)$ & $7028(4)$ & $24.0(10)$ \\
\hline C17_6 & $5234(4)$ & $2410.4(10)$ & $6742(4)$ & $33.9(12)$ \\
\hline C18_6 & $5763(4)$ & $2863.1(10)$ & $6371(3)$ & $25.7(10)$ \\
\hline C19_6 & $6137(3)$ & $3126.4(9)$ & $6660(3)$ & $22.3(9)$ \\
\hline C20_6 & $6348(3)$ & $3344.1(10)$ & $6168(3)$ & $26.6(9)$ \\
\hline C21_6 & $6740(3)$ & $3587.2(9)$ & $6906(3)$ & $22.8(8)$ \\
\hline O1_- 3 & $4854(2)$ & $5357.9(6)$ & $5916(2)$ & $26.5(6)$ \\
\hline $\mathrm{O} 2 \_3$ & $5869(3)$ & $5877.4(6)$ & $4169(3)$ & $24.8(7)$ \\
\hline O3_3 & $4024(3)$ & $5368.4(7)$ & $2480(3)$ & $35.1(8)$ \\
\hline O4_3 & $2658(3)$ & $4896.6(8)$ & $1960(3)$ & $38.8(9)$ \\
\hline N1_3 & $5512(3)$ & $5564.3(7)$ & $5052(3)$ & $21.3(7)$ \\
\hline N2_3 & $5102(3)$ & $5077.7(7)$ & $3727(3)$ & $22.3(7)$ \\
\hline C1_3 & $4881(3)$ & $5548.2(8)$ & $5437(3)$ & $20.9(8)$ \\
\hline $\mathrm{C} 2 \_3$ & $4285(3)$ & $5804.2(8)$ & $5129(3)$ & $22.0(8)$ \\
\hline C3_3 & $3542(3)$ & $5895.8(8)$ & $5299(3)$ & $23.4(8)$ \\
\hline C4_3 & $3103(3)$ & $6147.4(9)$ & $4885(3)$ & $26.9(10)$ \\
\hline $\mathrm{C} 5 \_3$ & $3398(4)$ & $6298.2(10)$ & $4319(4)$ & $25.8(10)$ \\
\hline C6_3 & 4151(4) & $6205.4(9)$ & $4162(4)$ & $23.7(10)$ \\
\hline C7_3 & $4575(3)$ & $5955.8(8)$ & $4571(3)$ & 20.2(9) \\
\hline C8_3 & $5392(3)$ & $5809.9(9)$ & $4542(3)$ & $20.6(9)$ \\
\hline C9_3 & $6242(3)$ & $5361.1(8)$ & $5166(3)$ & $22.4(8)$ \\
\hline C10_3 & $5989(3)$ & $5248.7(8)$ & $4158(3)$ & $22.0(8)$ \\
\hline C11_3 & $6875(3)$ & $5114.0(8)$ & $4184(4)$ & 27.4(9) \\
\hline $\mathrm{C} 12 \_3$ & $6842(4)$ & $4930.2(10)$ & $3571(4)$ & $35.9(10)$ \\
\hline C13_3 & $5042(3)$ & $4803.3(8)$ & $4001(3)$ & $20.6(8)$ \\
\hline C14_3 & $5771(3)$ & $4657.3(8)$ & $4788(3)$ & $21.6(8)$ \\
\hline C15_3 & $5554(3)$ & $4383.7(9)$ & $4862(3)$ & $22.3(8)$ \\
\hline C16_3 & $4658(4)$ & $4258.9(10)$ & $4200(4)$ & $24.9(10)$ \\
\hline $\mathrm{C} 17 \_3$ & $4482(4)$ & $3963.3(9)$ & $4324(4)$ & 31.1(11) \\
\hline $\mathrm{C} 18 \_3$ & $3915(4)$ & $4417.0(10)$ & $3434(4)$ & $25.6(10)$ \\
\hline C19_3 & $4129(3)$ & $4686.9(9)$ & $3350(3)$ & 24.1(9) \\
\hline $\mathrm{C} 20 \_3$ & $3525(4)$ & $4898.9(11)$ & $2630(4)$ & $29.4(11)$ \\
\hline C21_3 & $4217(4)$ & $5150.6(9)$ & 2901(4) & $26.5(9)$ \\
\hline O1_ $\overline{4}$ & $4378(2)$ & $5354.4(6)$ & $10109(2)$ & $30.3(7)$ \\
\hline $\mathrm{O} 2 \_4$ & $5801(3)$ & $5981.7(6)$ & $9053(3)$ & $25.9(7)$ \\
\hline O3_4 & $3686(2)$ & $5447.7(6)$ & $7109(2)$ & $27.5(7)$ \\
\hline O4_4 & 2411(3) & $4960.7(7)$ & $6637(2)$ & $28.8(7)$ \\
\hline N1_4 & $5274(3)$ & $5623.9(7)$ & $9621(3)$ & $22.5(8)$ \\
\hline $\mathrm{N} 2 \_4$ & $4872(3)$ & $5155.4(7)$ & $8264(3)$ & $19.4(7)$ \\
\hline C1_4 & $4519(3)$ & $5570.3(8)$ & $9834(3)$ & $21.8(8)$ \\
\hline $\mathrm{C} 2 \_4$ & $3958(3)$ & $5833.5(8)$ & $9635(3)$ & $22.6(8)$ \\
\hline C3_4 & $3160(3)$ & $5900.3(9)$ & $9745(3)$ & $26.2(9)$ \\
\hline C4_4 & $2770(4)$ & $6164.7(9)$ & $9466(4)$ & $26.6(10)$ \\
\hline C5_4 & $3167(4)$ & $6347.9(10)$ & $9081(4)$ & $25.8(10)$ \\
\hline C6 4 & $3972(4)$ & $6278.2(9)$ & $8969(4)$ & $23.6(10)$ \\
\hline
\end{tabular}




$\begin{array}{lllll}\text { C7_4 } & 4363(3) & 6018.0(9) & 9266(3) & 20.4(9) \\ \text { C8_4 } & 5224(3) & 5888.8(9) & 9277(3) & 20.8(9) \\ \text { C9_4 } & 6028(3) & 5432.6(9) & 9705(3) & 24.4(9) \\ \text { C10_4 } & 5751(3) & 5330.7(8) & 8672(3) & 21.1(8) \\ \text { C11_4 } & 6580(3) & 5194.7(7) & 8605(3) & 23.1(8) \\ \text { C12_4 } & 7512(3) & 5172.4(8) & 9315(3) & 27.5(9) \\ \text { C13_4 } & 4857(3) & 4884.1(8) & 8584(3) & 17.5(8) \\ \text { C14_4 } & 5629(3) & 4741.8(8) & 9358(3) & 21.0(8) \\ \text { C15_4 } & 5418(3) & 4475.5(9) & 9518(3) & 22.5(8) \\ \text { C16_4 } & 4498(3) & 4352.2(9) & 8944(3) & 20.4(9) \\ \text { C17_4 } & 4306(4) & 4065.0(9) & 9169(4) & 26.3(10) \\ \text { C18_4 } & 3732(4) & 4500.1(9) & 8177(3) & 21.8(9) \\ \text { C19_4 } & 3922(3) & 4766.8(9) & 8005(3) & 20.9(9) \\ \text { C20_4 } & 3285(3) & 4971.0(9) & 7285(3) & 20.6(9) \\ \text { C21_4 } & 3949(4) & 5227.3(10) & 7511(3) & 22.3(9)\end{array}$

Table S3. Anisotropic Displacement Parameters $\left(\AA^{2} \times 10^{3}\right)$ for $\mathbf{9 m j}$. The anisotropic displacement factor exponent takes the form: $-2 \pi^{2}\left[h^{2} a^{* 2} U_{11}+2 h k a * b * U_{12}+\ldots\right]$.

\begin{tabular}{lllllll} 
Attom & & $\mathbf{U}_{11}$ & \multicolumn{1}{c}{$\mathbf{U}_{22}$} & \multicolumn{1}{c}{$\mathbf{U}_{\mathbf{3 3}}$} & \multicolumn{1}{c}{$\mathbf{U}_{13}$} & $\mathbf{U}_{12}$ \\
O1_1 & $45(2)$ & $24.0(16)$ & $28.3(19)$ & $4.6(14)$ & $17.5(17)$ & $-1.8(14)$ \\
O2_1 & $33.2(19)$ & $34.2(19)$ & $19.4(17)$ & $2.8(14)$ & $11.5(14)$ & $-3.5(15)$ \\
O3_1 & $38(2)$ & $40(2)$ & $38(2)$ & $-2.9(17)$ & $24.0(19)$ & $4.0(17)$ \\
O4_1 & $33(2)$ & $55(2)$ & $45(2)$ & $-7.6(19)$ & $29.0(19)$ & $-6.0(18)$ \\
N1_1 & $30(2)$ & $20.2(18)$ & $18.0(19)$ & $-4.3(14)$ & $13.1(17)$ & $-4.9(15)$ \\
N2_1 & $31(2)$ & $25(2)$ & $27(2)$ & $-6.6(16)$ & $18.9(18)$ & $-5.7(17)$ \\
C1_1 & $32(3)$ & $24(2)$ & $21(2)$ & $-1.4(18)$ & $16(2)$ & $-7.7(19)$ \\
C2_1 & $30(3)$ & $22(2)$ & $19(2)$ & $-1.6(17)$ & $14(2)$ & $-7.3(19)$ \\
C3_1 & $38(3)$ & $31(3)$ & $24(2)$ & $-5(2)$ & $18(2)$ & $-15(2)$ \\
C4_1 & $42(3)$ & $32(3)$ & $38(3)$ & $-15(2)$ & $28(3)$ & $-19(2)$ \\
C5_1 & $38(3)$ & $27(2)$ & $38(3)$ & $-16(2)$ & $24(3)$ & $-12(2)$ \\
C6_1 & $32(3)$ & $24(2)$ & $33(3)$ & $-5(2)$ & $17(2)$ & $-5(2)$ \\
C7_1 & $27(2)$ & $25(2)$ & $18(2)$ & $-1.3(18)$ & $13(2)$ & $-7.8(19)$ \\
C8_1 & $27(2)$ & $26(2)$ & $22(2)$ & $-2.7(18)$ & $12(2)$ & $-7.2(18)$ \\
C9_1 & $29(2)$ & $26(2)$ & $26(2)$ & $-7.8(18)$ & $17(2)$ & $-7.1(18)$ \\
C10_1 & $30(3)$ & $32(2)$ & $24(2)$ & $-5.9(19)$ & $19(2)$ & $-8.4(19)$ \\
C11_1 & $39(3)$ & $34(2)$ & $22(2)$ & $-12(2)$ & $17(2)$ & $-14(2)$ \\
C12_1 & $38(3)$ & $21(2)$ & $31(3)$ & $-2.9(18)$ & $20(2)$ & $5.0(19)$ \\
C13_1 & $27(2)$ & $33(3)$ & $19(2)$ & $-6.2(18)$ & $12(2)$ & $-7(2)$ \\
C14_1 & $28(2)$ & $29(2)$ & $29(2)$ & $-6.5(19)$ & $17(2)$ & $-4.6(19)$ \\
C15_1 & $36(3)$ & $29(2)$ & $27(2)$ & $-6.3(19)$ & $19(2)$ & $-3.5(19)$ \\
C16_1 & $33(3)$ & $31(2)$ & $21(2)$ & $-3.6(17)$ & $12(2)$ & $-8.7(19)$ \\
C17_1 & $44(3)$ & $37(2)$ & $27(2)$ & $0.7(19)$ & $17(2)$ & $-6(2)$ \\
C18_1 & $28(2)$ & $41(3)$ & $19(2)$ & $-7.7(18)$ & $13.8(19)$ & $-13(2)$ \\
C19_1 & $32(3)$ & $34(3)$ & $24(2)$ & $-12(2)$ & $18(2)$ & $-11(2)$ \\
C20_1 & $30(3)$ & $39(3)$ & $29(3)$ & $-7(2)$ & $19(2)$ & $-5(2)$ \\
C21_1 & $33(3)$ & $36(3)$ & $23(2)$ & $-7(2)$ & $18(2)$ & $-4(2)$ \\
O1_2 & $45(2)$ & $30.3(18)$ & $34(2)$ & $7.8(16)$ & $19.6(18)$ & $-0.6(16)$ \\
O2_2 & $46(2)$ & $23.4(16)$ & $23.6(17)$ & $0.5(13)$ & $16.4(17)$ & $1.3(15)$ \\
O3_2 & $35(2)$ & $28.6(17)$ & $32.6(19)$ & $1.9(15)$ & $21.2(17)$ & $9.2(15)$
\end{tabular}




\begin{tabular}{|c|c|c|c|c|c|c|}
\hline O4_2 & $35(2)$ & $39.6(19)$ & $40(2)$ & $-0.8(16)$ & $27.3(18)$ & $1.5(16)$ \\
\hline N1_2 & $38(2)$ & $18.4(18)$ & $23(2)$ & $-3.6(15)$ & 19.3(19) & $-2.6(16)$ \\
\hline N2_2 & $27(2)$ & $19.5(18)$ & 22.2(19) & $-0.2(15)$ & 14.1(17) & $0.3(15)$ \\
\hline C1_2 & $35(3)$ & $27(2)$ & $23(2)$ & $0.8(19)$ & $17(2)$ & $-7(2)$ \\
\hline $\mathrm{C} 2 \_2$ & $33(3)$ & $28(2)$ & $21(2)$ & $-3.6(19)$ & $16(2)$ & $-10(2)$ \\
\hline C3_2 & $44(3)$ & $46(3)$ & $22(3)$ & $-8(2)$ & $18(2)$ & $-25(3)$ \\
\hline C4_2 & $52(4)$ & $47(3)$ & $35(3)$ & $-22(3)$ & $31(3)$ & $-30(3)$ \\
\hline C5_2 & $43(3)$ & $38(3)$ & $41(3)$ & $-26(3)$ & $31(3)$ & $-22(3)$ \\
\hline C6_2 & $36(3)$ & $28(3)$ & $34(3)$ & $-13(2)$ & $23(3)$ & $-10(2)$ \\
\hline C7_2 & $29(3)$ & $28(3)$ & $20(2)$ & $-6.6(19)$ & $16(2)$ & $-9(2)$ \\
\hline C8_2 & $29(2)$ & $21(2)$ & $21(2)$ & $-2.3(18)$ & $13(2)$ & $-4.8(18)$ \\
\hline C9_2 & $36(3)$ & $18.0(19)$ & $28(2)$ & $-2.3(17)$ & $22(2)$ & $-1.9(18)$ \\
\hline $\mathrm{C} 10 \_2$ & $34(3)$ & 17.6(19) & $23(2)$ & $3.5(16)$ & $17(2)$ & $3.3(17)$ \\
\hline C11_2 & $34(3)$ & $15.6(18)$ & $23(2)$ & $0.0(16)$ & $15(2)$ & $1.1(17)$ \\
\hline C12_2 & $48(3)$ & $25(2)$ & $21(2)$ & $0.1(18)$ & $18(2)$ & $7(2)$ \\
\hline C13_2 & $26(2)$ & $15(2)$ & $17(2)$ & $1.3(15)$ & $9.0(18)$ & $-0.3(17)$ \\
\hline C14_2 & $23(2)$ & $21(2)$ & $25(2)$ & $1.5(16)$ & $15.5(18)$ & $-1.7(16)$ \\
\hline C15_2 & $26(2)$ & $18(2)$ & $26(2)$ & $-0.3(17)$ & $15.4(18)$ & $2.5(17)$ \\
\hline C16_2 & $29(2)$ & $20.5(19)$ & $18(2)$ & $0.4(16)$ & $10.9(18)$ & $-3.0(17)$ \\
\hline C17_2 & $34(2)$ & 21.3(19) & $25(2)$ & $2.3(16)$ & $11.4(19)$ & $-2.1(17)$ \\
\hline C18_2 & $32(3)$ & $24(2)$ & $20(2)$ & $-1.1(16)$ & $16(2)$ & $-5.8(18)$ \\
\hline C19_2 & $26(2)$ & $23(2)$ & $21(2)$ & $-5.1(17)$ & $14.4(19)$ & $-5.5(18)$ \\
\hline $\mathrm{C} 20 \_2$ & $27(3)$ & $24(2)$ & $25(2)$ & $-2.7(19)$ & $14(2)$ & $-1.0(19)$ \\
\hline C21_2 & $29(3)$ & $25(2)$ & $19(2)$ & $-2.9(18)$ & $13(2)$ & $2(2)$ \\
\hline O1_5 & $31.4(18)$ & $24.3(17)$ & $28.1(17)$ & $-0.9(13)$ & $14.6(15)$ & $-6.0(13)$ \\
\hline $\mathrm{O} 2 \_5$ & $21.7(15)$ & $25.4(15)$ & $33.9(18)$ & $2.4(14)$ & $10.4(15)$ & $-1.5(12)$ \\
\hline O3_5 & $37.7(18)$ & $29.6(16)$ & $27.7(16)$ & $9.4(13)$ & $17.0(14)$ & $0.8(13)$ \\
\hline O4_5 & $37.2(18)$ & $41.6(18)$ & $19.4(15)$ & $4.8(13)$ & $16.5(14)$ & $5.7(14)$ \\
\hline N1_5 & 22.1(19) & $19.7(17)$ & $21.4(18)$ & $1.1(14)$ & $10.0(16)$ & $1.4(14)$ \\
\hline N2_5 & $25.6(18)$ & $21.0(17)$ & 18.1(18) & $3.4(14)$ & $11.8(16)$ & $2.3(14)$ \\
\hline C1_5 & $24(2)$ & $21(2)$ & $23(2)$ & $-4.5(17)$ & $14.5(19)$ & $-1.3(17)$ \\
\hline $\mathrm{C} 2{ }_{-}$ & $21(2)$ & $23(2)$ & $17(2)$ & $-1.4(17)$ & $10.4(17)$ & $3.0(17)$ \\
\hline C3_5 & $24(2)$ & $29(2)$ & $22(2)$ & $-4.2(18)$ & $11.9(18)$ & $2.1(18)$ \\
\hline C4_5 & $27(2)$ & $37(3)$ & $24(2)$ & $-3(2)$ & 12.1(19) & $10(2)$ \\
\hline C5_5 & $35(3)$ & $25(2)$ & $26(2)$ & $3.7(19)$ & $17(2)$ & $10(2)$ \\
\hline C6_5 & $32(3)$ & $22(2)$ & $26(2)$ & $1.5(17)$ & $15(2)$ & $4.0(18)$ \\
\hline C7_5 & $28(2)$ & $20(2)$ & $25(2)$ & $-2.7(17)$ & $15(2)$ & $2.5(18)$ \\
\hline C8_5 & $21(2)$ & $20(2)$ & $20(2)$ & $-1.1(16)$ & $9.9(18)$ & $1.4(16)$ \\
\hline C9_5 & $27(2)$ & 22.2(19) & $25(2)$ & $4.3(16)$ & $12.6(19)$ & $4.9(17)$ \\
\hline $\mathrm{C} 10 \_5$ & $24(2)$ & $17.9(18)$ & $25(2)$ & $0.8(16)$ & $12.2(19)$ & $-0.9(15)$ \\
\hline $\mathrm{C} 11 \_5$ & $26(2)$ & $18.3(17)$ & $26(2)$ & $1.3(15)$ & $11.5(18)$ & $1.4(15)$ \\
\hline C12_5 & $27(2)$ & $22(2)$ & $24(2)$ & 7.7(17) & 10.1(19) & $4.0(17)$ \\
\hline C13_5 & $20(2)$ & $21(2)$ & $21(2)$ & $2.9(16)$ & $11.2(18)$ & $6.2(16)$ \\
\hline C14_5 & $25(2)$ & $25(2)$ & $22(2)$ & $0.7(17)$ & 13.2(19) & $1.5(17)$ \\
\hline $\mathrm{C} 15 \_5$ & $25(2)$ & $24(2)$ & $26(2)$ & $4.9(18)$ & $14(2)$ & $1.3(18)$ \\
\hline C16_5 & $23(2)$ & $23(2)$ & $32(3)$ & $0.9(19)$ & $15(2)$ & $1.6(18)$ \\
\hline C17_5 & $28(2)$ & $26(2)$ & $39(3)$ & $-1(2)$ & $15(2)$ & $-1.2(19)$ \\
\hline C18_5 & $24(2)$ & $28(2)$ & $22(2)$ & $-2.7(17)$ & $12.6(18)$ & $0.6(17)$ \\
\hline C19_5 & $20(2)$ & $31(3)$ & $20(2)$ & $2.2(18)$ & 11.1(18) & $6.1(18)$ \\
\hline C20_5 & $23(2)$ & $30(2)$ & $21(2)$ & $2.0(17)$ & 11.4(18) & $4.2(17)$ \\
\hline
\end{tabular}




\begin{tabular}{|c|c|c|c|c|c|c|}
\hline C21_5 & $27(2)$ & $29(2)$ & $22(2)$ & $8.0(19)$ & 13.3(19) & $5.9(18)$ \\
\hline O1_ 6 & $27.3(17)$ & $21.4(16)$ & $27.0(17)$ & $-2.3(13)$ & $8.9(14)$ & $-6.0(13)$ \\
\hline O2_6 & $20.4(15)$ & $20.4(13)$ & $24.8(16)$ & $-1.0(12)$ & $7.8(13)$ & $-1.7(11)$ \\
\hline O3_6 & $39.3(18)$ & $30.3(16)$ & $35.9(18)$ & $12.9(14)$ & 24.1(16) & $2.3(14)$ \\
\hline O4_6 & $45(2)$ & $45.1(19)$ & $26.4(16)$ & $10.9(14)$ & $24.7(16)$ & $13.9(16)$ \\
\hline N1_6 & $21.7(18)$ & $14.4(16)$ & $21.0(18)$ & $1.0(14)$ & $9.7(15)$ & $-1.3(14)$ \\
\hline N2_6 & $25.3(19)$ & $18.8(17)$ & $22.7(19)$ & $5.2(15)$ & $13.6(16)$ & $2.0(14)$ \\
\hline C1_6 & $22(2)$ & $19(2)$ & $21(2)$ & $-2.0(17)$ & $10.4(18)$ & $1.4(17)$ \\
\hline $\mathrm{C} 2 \_6$ & $20(2)$ & $18(2)$ & 17.1(19) & $-2.7(16)$ & $8.0(17)$ & $0.3(16)$ \\
\hline C3_6 & $29(2)$ & $20(2)$ & $22(2)$ & $-5.2(17)$ & $10.1(18)$ & $1.7(18)$ \\
\hline C4_6 & $33(3)$ & $21(2)$ & $22(2)$ & $-0.9(17)$ & $13(2)$ & $6.6(19)$ \\
\hline C5_6 & $34(2)$ & $16.4(18)$ & $21(2)$ & $-0.5(15)$ & $13.0(19)$ & 4.6(17) \\
\hline C6_6 & $28(2)$ & $14.8(18)$ & $26(2)$ & $-2.4(15)$ & $13(2)$ & $1.0(16)$ \\
\hline C7_6 & $23(2)$ & $16.8(19)$ & $18(2)$ & $-2.0(16)$ & $9.8(18)$ & $1.0(16)$ \\
\hline C8_6 & $21(2)$ & $15.9(19)$ & $22(2)$ & $0.3(16)$ & $11.3(18)$ & $1.7(15)$ \\
\hline C9_6 & $26(2)$ & $15.9(18)$ & $24(2)$ & $2.8(15)$ & $14.7(18)$ & $2.5(16)$ \\
\hline C10_6 & $17.8(19)$ & 19.1(18) & $19(2)$ & $1.6(15)$ & $6.9(16)$ & $-1.7(15)$ \\
\hline C11_6 & $25(2)$ & $18.8(18)$ & $27(2)$ & $1.5(16)$ & 11.6(19) & $-3.7(16)$ \\
\hline C12_6 & $26(2)$ & $27(2)$ & $35(2)$ & $9.2(19)$ & $12(2)$ & $5.2(18)$ \\
\hline C13_6 & $20(2)$ & $18(2)$ & $20(2)$ & $3.1(16)$ & $9.6(17)$ & $4.4(15)$ \\
\hline C14_6 & $24(2)$ & $20(2)$ & $23(2)$ & $0.7(16)$ & $13.9(19)$ & $0.7(17)$ \\
\hline C15_6 & $27(2)$ & $22(2)$ & $26(2)$ & $1.8(18)$ & $15(2)$ & $0.0(17)$ \\
\hline C16_6 & $22(2)$ & $22(2)$ & $24(2)$ & $-3.7(18)$ & $9.5(18)$ & $1.3(17)$ \\
\hline C17_6 & $36(3)$ & $23(2)$ & $35(3)$ & $-5(2)$ & $12(2)$ & $-3(2)$ \\
\hline C18_6 & $26(2)$ & $28(2)$ & $20(2)$ & $0.9(19)$ & $9.4(19)$ & $6.2(19)$ \\
\hline C19_6 & $24(2)$ & $26(2)$ & $20(2)$ & $2.5(17)$ & $12.9(18)$ & $6.5(17)$ \\
\hline C20_6 & $27(2)$ & $35(2)$ & $21(2)$ & $9.9(19)$ & 14.7(19) & $14(2)$ \\
\hline C21_6 & $22(2)$ & $25(2)$ & $23(2)$ & $8.6(17)$ & $13.0(18)$ & $4.2(16)$ \\
\hline O1_3 & $33.2(16)$ & $21.8(13)$ & $29.6(16)$ & $2.9(12)$ & $19.5(14)$ & $-1.5(12)$ \\
\hline $\mathrm{O} 2 \_3$ & $30.1(16)$ & $19.5(15)$ & $33.1(17)$ & $1.7(13)$ & $22.0(14)$ & $0.4(13)$ \\
\hline O3_3 & $39(2)$ & $28.4(18)$ & $33.0(19)$ & $9.2(15)$ & $14.1(17)$ & $10.2(16)$ \\
\hline O4_3 & $27.5(19)$ & $46(2)$ & $27(2)$ & $-4.9(16)$ & $2.2(16)$ & $3.3(16)$ \\
\hline N1_3 & $26.2(18)$ & $13.8(15)$ & $27.5(19)$ & $0.7(14)$ & $16.2(17)$ & $2.6(13)$ \\
\hline N2_3 & $24.2(18)$ & $18.7(17)$ & $21.6(18)$ & $0.5(14)$ & $9.7(16)$ & $5.1(14)$ \\
\hline C1_3 & $25(2)$ & 17.1(18) & $22(2)$ & $-4.3(16)$ & $12.9(18)$ & $-4.9(15)$ \\
\hline C2 23 & $25(2)$ & $16.7(18)$ & $23(2)$ & $-5.1(16)$ & $10.5(18)$ & $-2.0(16)$ \\
\hline C3_3 & $25(2)$ & $21.9(19)$ & $26(2)$ & $-3.9(17)$ & $14.7(18)$ & $-2.1(16)$ \\
\hline C4_3 & $24(2)$ & $26(2)$ & $30(2)$ & $-11.8(19)$ & $14(2)$ & $-2.4(18)$ \\
\hline C5_3 & $29(2)$ & $18(2)$ & $31(3)$ & $-5.3(18)$ & $14(2)$ & $1.4(17)$ \\
\hline C6_3 & $29(2)$ & $14(2)$ & $27(2)$ & $-3.4(17)$ & $13(2)$ & $-0.8(17)$ \\
\hline C7_3 & $21(2)$ & $14.4(19)$ & $27(2)$ & $-4.8(16)$ & $13.3(19)$ & $-2.6(15)$ \\
\hline C8_3 & $23(2)$ & $12.6(18)$ & $25(2)$ & $-2.3(16)$ & $11.2(18)$ & $-2.8(16)$ \\
\hline C9_3 & $23(2)$ & $17.7(18)$ & $26(2)$ & $2.4(16)$ & $12.2(18)$ & $3.7(15)$ \\
\hline C10_3 & $26(2)$ & $16.5(18)$ & $28(2)$ & $0.9(16)$ & $17.3(18)$ & $1.8(15)$ \\
\hline $\mathrm{C} 11 \_3$ & $30(2)$ & $21.8(19)$ & $35(2)$ & $2.1(18)$ & $20(2)$ & $3.4(17)$ \\
\hline C12_3 & $37(3)$ & $42(3)$ & $34(2)$ & $1(2)$ & $21(2)$ & $14(2)$ \\
\hline C13_3 & $22(2)$ & $21(2)$ & $24(2)$ & $-2.4(16)$ & 15.3(19) & $0.8(16)$ \\
\hline C14_3 & $23(2)$ & $20(2)$ & $24(2)$ & $-1.7(16)$ & $13.0(18)$ & $-0.3(16)$ \\
\hline C15_3 & $27(2)$ & $20.0(19)$ & $23(2)$ & $1.2(16)$ & $14.5(18)$ & $1.2(16)$ \\
\hline C16_3 & $28(2)$ & $25(2)$ & $27(2)$ & $-4.8(18)$ & $18(2)$ & $-4.9(18)$ \\
\hline
\end{tabular}




$\begin{array}{lllllll}\text { C17_3 } & 42(3) & 24(2) & 38(3) & -6(2) & 28(2) & -9(2) \\ \text { C18_3 } & 27(2) & 27(2) & 25(2) & -8.9(18) & 15(2) & -3.2(19) \\ \text { C19_3 } & 23(2) & 29(2) & 21(2) & -6.2(18) & 11.7(19) & 0.7(18) \\ \text { C20_3 } & 25(2) & 35(3) & 23(2) & -6(2) & 8(2) & 8(2) \\ \text { C21_3 } & 28(2) & 28(2) & 25(2) & 1.1(18) & 14(2) & 10.3(19) \\ \text { O1_4 } & 36.6(17) & 22.0(14) & 38.7(18) & 4.3(13) & 23.6(16) & -2.1(12) \\ \text { O2_4 } & 27.1(16) & 26.5(17) & 30.7(17) & -3.8(13) & 19.3(14) & -5.2(13) \\ \text { O3_4 } & 31.3(17) & 24.6(15) & 29.4(17) & 9.4(13) & 17.3(15) & 7.5(13) \\ \text { O4_4 } & 22.8(17) & 33.9(17) & 23.6(17) & 1.4(13) & 6.8(14) & 4.4(13) \\ \text { N1_4 } & 26.8(19) & 17.6(18) & 23.9(19) & -2.9(14) & 13.3(16) & -2.4(14) \\ \text { N2_4 } & 20.6(18) & 18.0(16) & 20.2(18) & 1.7(13) & 10.4(16) & -0.8(14) \\ \text { C1_4 } & 26(2) & 19.2(18) & 22(2) & -2.5(16) & 13.7(18) & -1.9(16) \\ \text { C2_4 } & 26(2) & 18.9(18) & 21(2) & -0.6(16) & 11.0(18) & -2.2(16) \\ \text { C3_4 } & 30(2) & 21.3(19) & 32(2) & -5.3(18) & 19(2) & -4.4(17) \\ \text { C4_4 } & 27(2) & 24(2) & 33(3) & -6.4(19) & 17(2) & 0.1(18) \\ \text { C5_4 } & 31(2) & 17(2) & 26(2) & -2.9(17) & 12(2) & 0.7(18) \\ \text { C6_4 } & 32(2) & 16(2) & 24(2) & -2.3(16) & 15(2) & -4.3(17) \\ \text { C7_4 } & 25(2) & 18(2) & 16(2) & -4.0(16) & 8.9(18) & -3.9(17) \\ \text { C8_4 } & 22(2) & 21(2) & 20(2) & -4.9(16) & 10.2(18) & -6.0(17) \\ \text { C944 } & 22(2) & 25(2) & 27(2) & -1.7(17) & 13.3(19) & 0.7(16) \\ \text { C10_4 } & 24(2) & 16.3(18) & 25(2) & -1.7(15) & 13.7(18) & -1.4(15) \\ \text { C11_4 } & 29(2) & 13.0(16) & 31(2) & 0.2(16) & 18.4(19) & -0.2(15) \\ \text { C12_4 } & 28(2) & 21.2(19) & 38(2) & 2.6(17) & 20(2) & 3.4(17) \\ \text { C13_4 } & 19.9(19) & 18.2(19) & 17.6(19) & -1.0(15) & 11.7(17) & 0.1(15) \\ \text { C14_4 } & 23(2) & 23(2) & 19(2) & 1.7(16) & 11.3(18) & 0.0(16) \\ \text { C15_4 } & 26(2) & 22(2) & 20(2) & 0.7(16) & 11.9(18) & 0.2(17) \\ \text { C16_4 } & 22(2) & 19(2) & 23(2) & -0.8(16) & 13.9(19) & -0.7(17) \\ \text { C17_4 } & 33(2) & 20(2) & 34(2) & 2.1(18) & 22(2) & -0.3(18) \\ \text { C18_4 } & 25(2) & 22(2) & 23(2) & -3.5(16) & 14.8(19) & -1.2(17) \\ \text { C19_4 } & 21(2) & 24(2) & 19(2) & 0.7(17) & 10.6(18) & 3.2(17) \\ \text { C20_4 } & 21(2) & 27(2) & 15(2) & -0.5(16) & 9.7(18) & 2.5(17) \\ \text { C21_4 } & 26(2) & 26(2) & 19(2) & 2.5(18) & 14.5(19) & 6.8(18)\end{array}$

Table S4. Bond Lengths for $9 \mathbf{m j}$.

\begin{tabular}{|c|c|c|c|c|c|}
\hline Atom & Atom & Length/Å & Atom & Atom & Length/Å \\
\hline O1 1 & $\mathrm{C} 1 \quad 1$ & $1.217(7)$ & O1 6 & $\mathrm{C} 16$ & $1.211(6)$ \\
\hline O2_1 & C8_1 & $1.208(7)$ & O2_6 & C8_6 & $1.230(6)$ \\
\hline O3_1 & C21_1 & $1.203(8)$ & O3_6 & C21_6 & $1.209(5)$ \\
\hline O4_1 & C20_1 & $1.215(7)$ & O4_6 & C20_6 & $1.221(5)$ \\
\hline N1_1 & C1_1 & $1.393(6)$ & N1_6 & C1_6 & $1.404(6)$ \\
\hline N1_1 & C8_1 & $1.401(7)$ & N1_6 & C8_6 & $1.395(6)$ \\
\hline N1_1 & C9_1 & $1.449(6)$ & N1_6 & C9_6 & $1.457(6)$ \\
\hline N2_1 & C10_1 & $1.465(6)$ & N2_6 & C10_6 & $1.458(5)$ \\
\hline N2_1 & C13_1 & $1.405(7)$ & N2_6 & C13_6 & $1.415(6)$ \\
\hline N2_1 & C21_1 & $1.386(7)$ & $\mathrm{N} 2 \_6$ & C21_6 & $1.371(5)$ \\
\hline C1_1 & $\mathrm{C} 2 \_1$ & $1.486(8)$ & C1_6 & C2_6 & $1.501(6)$ \\
\hline $\mathrm{C} 2 \_1$ & C3_1 & $1.386(8)$ & $\mathrm{C} 2 \_6$ & C3_6 & $1.390(7)$ \\
\hline C2_1 & C7_1 & $1.391(8)$ & C2_6 & C7_6 & $1.399(6)$ \\
\hline C3_1 & C4_1 & $1.383(9)$ & C3_6 & C4_6 & $1.398(6)$ \\
\hline
\end{tabular}




\begin{tabular}{|c|c|c|c|c|c|}
\hline C4_1 & C5_1 & $1.386(10)$ & C4_6 & C5_6 & $1.395(7)$ \\
\hline $\mathrm{C} 5 \_1$ & C6_1 & $1.394(8)$ & C5_6 & C6_6 & $1.395(6)$ \\
\hline C6_1 & $\mathrm{C} 7 \_1$ & $1.377(8)$ & C6_6 & C7_6 & $1.376(6)$ \\
\hline C7_1 & C8 1 & $1.478(7)$ & C7 6 & C8 6 & $1.474(7)$ \\
\hline C9_1 & C10_1 & $1.528(7)$ & C9_6 & C10_6 & $1.536(6)$ \\
\hline $\mathrm{C} 10 \_1$ & C11_1 & $1.496(6)$ & C10_6 & C11_6 & $1.516(6)$ \\
\hline C11_1 & C12_1 & $1.317(7)$ & C11_6 & C12_6 & $1.310(6)$ \\
\hline C13_1 & C14_1 & $1.385(8)$ & C13 6 & C14_6 & $1.368(7)$ \\
\hline C13_1 & C19_1 & $1.406(7)$ & C13_6 & C19_6 & $1.399(6)$ \\
\hline C14_1 & C15_1 & $1.399(7)$ & C14_6 & C15_6 & $1.397(7)$ \\
\hline C15_1 & C16_1 & $1.403(7)$ & C15_6 & C16_6 & $1.391(8)$ \\
\hline C16_1 & C17_1 & $1.511(6)$ & C16_6 & C17_6 & $1.512(6)$ \\
\hline C16_1 & C18_1 & $1.398(7)$ & C16_6 & C18_6 & $1.395(7)$ \\
\hline C18_1 & C19_1 & $1.378(8)$ & C18_6 & C19_6 & $1.397(6)$ \\
\hline C19_1 & C20_1 & $1.459(9)$ & C19_6 & C20_6 & $1.446(6)$ \\
\hline C20_1 & C21_1 & $1.560(8)$ & C20_6 & C21_6 & $1.555(7)$ \\
\hline O1_2 & $\mathrm{C} 1 \_2$ & $1.212(7)$ & O1_3 & C1_3 & $1.211(5)$ \\
\hline $\mathrm{O} 2 \_2$ & C8_2 & $1.209(7)$ & $\mathrm{O} 2 \_3$ & C8_3 & $1.199(6)$ \\
\hline O3_2 & C21_2 & $1.210(7)$ & O3_3 & C21_3 & $1.211(6)$ \\
\hline O4_2 & C20_2 & $1.210(7)$ & O4_3 & $\mathrm{C} 20 \_3$ & $1.229(6)$ \\
\hline N1_2 & $\mathrm{C} 1 \_2$ & $1.404(6)$ & N1_3 & C1_3 & $1.387(5)$ \\
\hline N1_2 & C8_2 & $1.400(6)$ & N1_3 & C8_3 & $1.404(5)$ \\
\hline N1_2 & C9_2 & $1.445(6)$ & N1_3 & C9_3 & $1.456(5)$ \\
\hline N2_2 & C10_2 & $1.461(6)$ & N2_3 & C10_3 & $1.464(5)$ \\
\hline N2_2 & C13_2 & $1.413(6)$ & N2_3 & C13_3 & $1.429(5)$ \\
\hline N2_2 & C21_2 & $1.375(7)$ & N2_3 & C21_3 & $1.386(6)$ \\
\hline C1_2 & $\mathrm{C} 2 \_2$ & $1.488(8)$ & C1_3 & $\mathrm{C} 2 \_3$ & $1.493(6)$ \\
\hline $\mathrm{C} 2 \_2$ & C3_2 & $1.380(8)$ & C2_3 & C3_3 & $1.385(6)$ \\
\hline $\mathrm{C} 2 \_2$ & C7_2 & $1.389(8)$ & 233 & C7_3 & $1.383(6)$ \\
\hline C3_2 & C4_2 & $1.388(10)$ & C3_3 & C4_3 & $1.403(6)$ \\
\hline C4_2 & C5_2 & $1.376(11)$ & C4_3 & C5_3 & $1.395(7)$ \\
\hline C5_2 & C6_2 & $1.389(8)$ & C5_3 & C6_3 & $1.390(7)$ \\
\hline C6_2 & C7_2 & $1.377(8)$ & C6_3 & C7_3 & $1.387(6)$ \\
\hline C7_2 & C8_2 & $1.474(7)$ & C7_3 & C8_3 & $1.481(6)$ \\
\hline C9_2 & C10_2 & $1.541(7)$ & C9_3 & C10_3 & $1.522(6)$ \\
\hline C10_2 & C11_2 & $1.499(6)$ & C10_3 & C11_3 & $1.514(6)$ \\
\hline C11_2 & C12_2 & $1.322(7)$ & C11_3 & C12_3 & $1.297(6)$ \\
\hline C13_2 & C14_2 & $1.386(7)$ & C13_3 & C14_3 & $1.386(6)$ \\
\hline C13_2 & C19_2 & $1.398(7)$ & C13_3 & C19_3 & $1.394(6)$ \\
\hline C14_2 & C15_2 & $1.396(6)$ & C14_3 & C15_3 & $1.403(6)$ \\
\hline C15_2 & C16_2 & $1.404(6)$ & C15_3 & C16_3 & $1.399(6)$ \\
\hline C16_2 & C17_2 & $1.504(6)$ & C16_3 & C17_3 & $1.506(6)$ \\
\hline C16_2 & C18_2 & $1.399(7)$ & C16_3 & C18_3 & $1.412(7)$ \\
\hline C18_2 & C19_2 & $1.373(7)$ & C18_3 & C19_3 & $1.388(6)$ \\
\hline C19_2 & $\mathrm{C} 20 \_2$ & $1.471(8)$ & C19_3 & $\mathrm{C} 20 \_3$ & $1.475(6)$ \\
\hline C20_2 & C21_2 & $1.558(7)$ & C20_3 & C21_3 & $1.554(7)$ \\
\hline O1_5 & C1_5 & $1.209(6)$ & O1_4 & C1_4 & $1.203(5)$ \\
\hline $\mathrm{O} 2 \_5$ & C8_5 & $1.227(6)$ & $\mathrm{O} 2 \_4$ & C8_4 & $1.205(6)$ \\
\hline O3_5 & C21_5 & $1.206(5)$ & O3_4 & C21_4 & $1.214(6)$ \\
\hline O4_5 & $\mathrm{C} 20 \_5$ & $1.215(5)$ & O4_4 & $\mathrm{C} 2 \mathrm{C}_{-} 4$ & $1.229(6)$ \\
\hline
\end{tabular}




\begin{tabular}{|c|c|c|c|c|c|}
\hline N1_5 & C1_5 & $1.408(7)$ & N1_4 & C1_4 & $1.399(5)$ \\
\hline N1_5 & C8_5 & $1.392(6)$ & N1_4 & C8_4 & $1.393(5)$ \\
\hline N1_5 & C9_5 & $1.450(6)$ & N1_4 & C9_4 & $1.458(6)$ \\
\hline N2_5 & C10_5 & $1.470(5)$ & $\mathrm{N} 2 \_4$ & C10_4 & $1.468(5)$ \\
\hline N2_5 & C13_5 & $1.422(6)$ & N2_4 & C13_4 & $1.426(5)$ \\
\hline N2_5 & C21_5 & $1.369(5)$ & $\mathrm{N} 2 \_4$ & C21_4 & $1.375(6)$ \\
\hline C1_5 & $\mathrm{C} 2 \_5$ & $1.498(6)$ & C1_4 & $\mathrm{C} 2 \_4$ & $1.503(6)$ \\
\hline $\mathrm{C} 2-5$ & C3_5 & $1.389(6)$ & $\mathrm{C} 2 \_4$ & C3_ 4 & $1.378(6)$ \\
\hline $\mathrm{C} 2 \_5$ & C7_5 & $1.400(7)$ & $\mathrm{C} 2 \_4$ & C7_4 & $1.383(6)$ \\
\hline C3_5 & C4_5 & $1.400(7)$ & C3_4 & C4_4 & $1.407(6)$ \\
\hline C4_5 & C5_5 & $1.399(7)$ & C4 44 & C5 4 & $1.387(7)$ \\
\hline C5_5 & C6_5 & $1.388(7)$ & C5_4 & C6 4 & $1.394(7)$ \\
\hline C6_5 & C7_5 & $1.384(7)$ & C6_4 & C7_4 & $1.391(6)$ \\
\hline C7_5 & C8_5 & $1.474(7)$ & C7_4 & C8_4 & $1.479(6)$ \\
\hline C9 5 & C10_5 & $1.530(6)$ & C9 4 & C10_4 & $1.531(6)$ \\
\hline C10_5 & C11_5 & $1.512(6)$ & C10_4 & C11_4 & $1.504(6)$ \\
\hline C11_5 & C12_5 & $1.320(6)$ & C11_4 & C12_4 & $1.322(6)$ \\
\hline C13_5 & C14_5 & $1.368(7)$ & C13_4 & C14_4 & $1.395(6)$ \\
\hline C13_5 & C19_5 & $1.400(6)$ & C13_4 & C19_4 & $1.401(6)$ \\
\hline C14_5 & C15_5 & $1.407(7)$ & C14_4 & C15_4 & $1.400(6)$ \\
\hline C15_5 & C16_5 & $1.381(8)$ & C15_4 & C16_4 & $1.395(6)$ \\
\hline C16_5 & C17_5 & $1.515(6)$ & C16_4 & C17_4 & $1.518(6)$ \\
\hline C16_5 & C18_5 & $1.399(7)$ & C16_4 & C18_4 & $1.402(6)$ \\
\hline C18_5 & C19_5 & $1.391(6)$ & C18_4 & C19_4 & $1.397(6)$ \\
\hline C19_5 & C20_5 & $1.448(6)$ & C19_4 & $\mathrm{C} 20 \_4$ & $1.462(6)$ \\
\hline $\mathrm{C} 20 \_5$ & C21_5 & $1.556(7)$ & C20_4 & C21_4 & $1.553(7)$ \\
\hline
\end{tabular}

Table S5. Bond Angles for $\mathbf{9 m j}$.

\begin{tabular}{|c|c|c|c|c|c|c|c|}
\hline m & tom & m & $1^{\circ}$ & tom & tom & Itom & \\
\hline 111 & N1_1 & C8_1 & $2.1(4)$ & $1 \_6$ & N1_6 & C9_6 & \\
\hline 1_1 & N1_1 & C9_1 & 0. & 3_6 & -0 & - & \\
\hline B_ 1 & N1_1 & C9_1 & & 8 & & C9 & \\
\hline 13_1 & N2_1 & C10_1 & 1 & 13_6 & N2_6 & 10_6 & \\
\hline 211 & $\mathrm{~N} 2$ & & & & N2_6 & & \\
\hline 21 & N2_1 & C & & & N2_6 & & \\
\hline $1 \_1$ & $\mathrm{C} 1 \_1$ & N1_1 & 12 & U & $\mathrm{C} 1 \_6$ & -0 & (4) \\
\hline 1_1 & C1_1 & $\mathrm{C} 2$ & & -6 & C1_6 & 6 & \\
\hline 11 & $\mathrm{C} 1 \_1$ & $\mathrm{C} 2$ & & & $\mathrm{C} 1 \_6$ & & \\
\hline 3_1 & C2_1 & $\mathrm{C} 1 \_1$ & & 6 & C2_6 & 6 & \\
\hline $3 \_1$ & C2 1 & C7 1 & & 6 & 6 & 6 & \\
\hline 7_1 & C2_1 & $\mathrm{C} 1$ & & 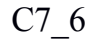 & C2_6 & 6 & \\
\hline $4 \_1$ & C3 1 & C2 1 & & 6 & C3_6 & +6 & \\
\hline $3 \_1$ & C4_1 & $\mathrm{C} 5 \_1$ & & C5_6 & C4_6 & C3_6 & 12 \\
\hline $4 \_1$ & C5 1 & C6_1 & & & C5 6 & $4 \_6$ & \\
\hline 7_1 & C6_ & C5 & & $\mathrm{C}^{\prime}$ & C6_6 & 5_6 & \\
\hline 21 & C7 1 & C8_1 & & $\mathrm{C} 2{ }^{6}$ & C7_6 & 86 & \\
\hline $6 \_1$ & C7_1 & C2_1 & 122. & C6_6 & C7_6 & C2_6 & $121.6(4)$ \\
\hline 61 & & & & & C7 6 & & 1. \\
\hline $2 \_1$ & C8_1 & N1_1 & 124 & O2_6 & C8_6 & N1_6 & 124.2 \\
\hline
\end{tabular}




\begin{tabular}{|c|c|c|c|c|c|c|c|}
\hline $2 \_1$ & C8_1 & C7_1 & $129.6(5)$ & O2_6 & C8_6 & C7_6 & $129.4(4)$ \\
\hline $1 \_1$ & C8_1 & C7_1 & $106.0(4)$ & N1_6 & C8_6 & C7_6 & $106.4(4)$ \\
\hline N1_1 & C9_1 & C10_1 & $110.7(4)$ & N1_6 & C9_6 & C10_6 & $110.6(3)$ \\
\hline N2 1 & $\mathrm{C} 10 \_1$ & C9 1 & $112.2(4)$ & N2 6 & $\mathrm{C} 10 \_6$ & C9 6 & $110.0(3)$ \\
\hline $2 \_1$ & C10_1 & C11_1 & $110.5(4)$ & N2_6 & C10_6 & C11_6 & $113.5(3)$ \\
\hline C11_1 & $\mathrm{C} 10 \_1$ & C9 1 & $114.8(4)$ & C11_6 & $\mathrm{C} 10 \_6$ & C9 6 & 113.1(3) \\
\hline C12_1 & C11_1 & C10_1 & $127.5(5)$ & C12_6 & C11_6 & C10_6 & $125.5(4)$ \\
\hline N2 1 & $\mathrm{C} 13 \_1$ & C19_1 & $111.0(5)$ & C14_6 & $\mathrm{C} 13 \_6$ & N2_ 6 & $128.2(4)$ \\
\hline C14_1 & C13_1 & N2_1 & $129.2(5)$ & C14_6 & C13_6 & C19_6 & $120.7(4)$ \\
\hline C14_1 & C13_1 & C19_1 & $119.7(5)$ & C19_6 & C13_6 & N2_6 & 111.1(4) \\
\hline C13_1 & C14_1 & C15_1 & $118.4(5)$ & C13_6 & C14_6 & C15_6 & $118.3(5)$ \\
\hline C14_1 & $\mathrm{C} 15 \_1$ & C16_1 & $122.3(5)$ & C16_6 & $\mathrm{C} 15 \_6$ & C14_6 & $122.5(5)$ \\
\hline C15_1 & C16_1 & C17_1 & $119.9(5)$ & C15_6 & C16_6 & C17_6 & $120.2(5)$ \\
\hline C18_1 & C16_1 & C15_1 & $118.2(4)$ & C15_6 & C16_6 & C18_6 & $118.4(4)$ \\
\hline C18_1 & C16_1 & C17_1 & $121.9(4)$ & C18_6 & C16_6 & C17_6 & $121.4(5)$ \\
\hline C19_1 & C18_1 & C16_1 & $119.8(4)$ & C16_6 & C18_6 & C19_6 & $119.5(4)$ \\
\hline C13_1 & C19_1 & C20_1 & $106.8(5)$ & C13_6 & C19_6 & C20_6 & $106.7(4)$ \\
\hline C18_1 & C19_1 & C13_1 & $121.4(5)$ & C18_6 & C19_6 & C13_6 & $120.5(4)$ \\
\hline C18_1 & C19_1 & C20_1 & $131.8(5)$ & C18_6 & C19_6 & C20_6 & $132.9(4)$ \\
\hline O4_1 & C20_1 & C19_1 & $131.3(6)$ & O4_6 & C20_6 & C19_6 & $130.4(5)$ \\
\hline O4_1 & C20_1 & C21_1 & $122.5(6)$ & O4_6 & C20_6 & C21_6 & $123.1(4)$ \\
\hline C19_1 & C20_1 & C21_1 & $106.3(5)$ & C19_6 & C20_6 & C21_6 & $106.4(4)$ \\
\hline O3_1 & C21_1 & N2_1 & $128.1(5)$ & O3_6 & C21_6 & N2_6 & $127.4(4)$ \\
\hline O3_1 & C21_1 & C20_1 & $127.4(5)$ & O3_6 & C21_6 & C20_6 & $127.7(4)$ \\
\hline N2_1 & C21_1 & C20_1 & $104.5(5)$ & N2_6 & C21_6 & C20_6 & $105.0(3)$ \\
\hline C1_2 & N1_2 & C9_2 & $125.7(4)$ & C1_3 & N1_3 & C8_3 & $111.8(3)$ \\
\hline C8_2 & N1_2 & C1_2 & $112.1(4)$ & C1_3 & N1_3 & C9_3 & $126.1(3)$ \\
\hline C8_2 & N1_2 & C9_2 & $122.2(4)$ & C8_3 & N1_3 & C9_3 & $122.1(4)$ \\
\hline C13_2 & N2_2 & C10_2 & $125.1(4)$ & C13_3 & N2_3 & C10_3 & $126.2(4)$ \\
\hline C21_2 & N2_2 & C10_2 & $123.1(4)$ & C21_3 & N2_3 & C10_3 & $123.6(4)$ \\
\hline C21_2 & $\mathrm{N} 2 \_2$ & C13_2 & $111.7(4)$ & C21_3 & N2_3 & C13_3 & $109.8(4)$ \\
\hline O1_2 & $\mathrm{C} 1 \_2$ & N1_2 & $124.5(5)$ & O1_3 & $\mathrm{C} 1 \_3$ & N1_3 & $124.2(4)$ \\
\hline O1_2 & $\mathrm{C} 1 \_2$ & $\mathrm{C} 2 \_2$ & $130.5(5)$ & O1_3 & $\mathrm{C} 1 \_3$ & $\mathrm{C} 2 \_3$ & $129.7(4)$ \\
\hline N1_2 & C1_2 & C2_2 & $105.0(4)$ & N1_3 & C1_3 & C2_3 & $106.0(3)$ \\
\hline C3_2 & C2 2 & C1_2 & $130.3(6)$ & C3_3 & $\mathrm{C} 2 \_3$ & C1_3 & $131.2(4)$ \\
\hline C3_2 & C2_2 & C7_2 & 121.1(6) & C7_3 & $\mathrm{C} 2 \_3$ & C1_3 & $107.9(4)$ \\
\hline C7_2 & C2 2 & C1_2 & $108.7(5)$ & C7_3 & $\mathrm{C} 2 \_3$ & C3_3 & $120.9(4)$ \\
\hline C2_2 & C3_2 & C4_2 & $116.2(6)$ & C2_3 & C3_3 & C4_3 & $117.4(4)$ \\
\hline C5_2 & C4_2 & C3_2 & $122.7(6)$ & C5_3 & C4_3 & C3_3 & $121.3(4)$ \\
\hline C4_2 & C5_2 & C6_2 & $121.0(6)$ & C6_3 & C5_3 & C4_3 & $120.8(4)$ \\
\hline C7_2 & C6_2 & $\mathrm{C} 5 \_2$ & $116.4(6)$ & C7_3 & C6_3 & $\mathrm{C} 5 \_3$ & $117.3(4)$ \\
\hline C2_2 & C7_2 & C8_2 & $108.2(5)$ & C2_3 & C7_3 & C6_3 & $122.3(4)$ \\
\hline C6_2 & C7_2 & $\mathrm{C} 2 \_2$ & $122.5(5)$ & C2_3 & C7_3 & C8_3 & $108.4(4)$ \\
\hline C6_2 & C7_2 & C8_2 & $129.3(5)$ & C6_3 & C7_3 & C8_3 & $129.3(4)$ \\
\hline $\mathrm{O} 2 \_2$ & C8_2 & N1_2 & $124.1(5)$ & $\mathrm{O} 2 \_3$ & $\mathrm{C} 8 \_3$ & N1_3 & $124.9(4)$ \\
\hline $\mathrm{O} 2 \_2$ & C8_2 & C7_2 & $129.8(5)$ & $\mathrm{O} 2 \_3$ & C8_3 & C7_3 & $129.3(4)$ \\
\hline N1_2 & C8_2 & C7_2 & $106.1(4)$ & N1_3 & C8_3 & C7_3 & $105.8(4)$ \\
\hline N1_2 & C9_2 & C10_2 & $110.6(4)$ & N1_3 & C9_3 & C10_3 & $109.8(3)$ \\
\hline N2_2 & C10_2 & C9_2 & 111.1(4) & N2_3 & C10_3 & C9_3 & $112.4(3)$ \\
\hline $\mathrm{N} 2 \_2$ & C10_2 & C11_2 & $110.1(4)$ & N2_3 & C10_3 & C11_3 & $113.8(3)$ \\
\hline
\end{tabular}




\begin{tabular}{|c|c|c|c|c|c|c|c|}
\hline $11 \_2$ & $10 \_2$ & C9_2 & $115.1(4)$ & C11_3 & C10_3 & C9 3 & $111.6(4)$ \\
\hline C12_2 & C11_2 & C10_2 & $125.4(5)$ & $\mathrm{C} 12 \_3$ & C11_3 & C10_3 & $125.7(4)$ \\
\hline C14_2 & C13_2 & $\mathrm{N} 2 \_\overline{2}$ & $129.1(4)$ & C14_3 & C13_3 & $\mathrm{N} 2 \_\overline{3}$ & $127.2(4)$ \\
\hline C14_2 & C13_2 & C19_2 & $120.1(4)$ & C14_3 & C13_3 & C19_3 & $121.5(4)$ \\
\hline C19_2 & $\mathrm{C} 132$ & $\mathrm{~N} 22$ & $110.8(5)$ & $\mathrm{C} 193$ & $\mathrm{C} 13 \_3$ & N2 3 & $111.3(4)$ \\
\hline C13_2 & C14_2 & C15_2 & $117.8(4)$ & C13_3 & C14_3 & C15_3 & $116.3(4)$ \\
\hline C14 2 & $\mathrm{C} 152$ & $\mathrm{C} 162$ & $122.8(4)$ & $\mathrm{C} 163$ & $\mathrm{C} 153$ & C14 3 & $123.4(4)$ \\
\hline C15_2 & C16_2 & C17_2 & $120.6(4)$ & C15_3 & C16_3 & C17_3 & $120.4(5)$ \\
\hline C18_2 & $\mathrm{C} 162$ & $\mathrm{C} 15 \_2$ & $117.8(4)$ & $\mathrm{C} 153$ & $\mathrm{C} 16 \_3$ & C18_3 & 3(4) \\
\hline C18_2 & C16_2 & C17_2 & $121.6(4)$ & C18_3 & C16_3 & C17_3 & $120.8(4)$ \\
\hline C19_2 & C18_2 & C16_2 & 119.8 & $19 \_3$ & C18_3 & C16_3 & $118.0(4)$ \\
\hline C13_2 & C19_2 & C20_2 & $106.8(5)$ & C13_3 & C19_3 & C20_3 & $107.4(4)$ \\
\hline C18_2 & C19 2 & $\mathrm{C} 1322$ & $121.7(5)$ & 83 & $\mathrm{C} 193$ & C13_3 & (4) \\
\hline C18_2 & C19_2 & C20_2 & $131.5(5)$ & C18_3 & C19_3 & $\mathrm{C} 20 \_3$ & $130.7(5)$ \\
\hline O4_2 & C20_2 & C19_2 & 131. & O4_3 & C20_3 & C19_3 & 13 \\
\hline O4_2 & C20_2 & C21_2 & $122.4(5)$ & O4_3 & C20_3 & C21_3 & $124.0(5)$ \\
\hline C19 2 & $\mathrm{C} 202$ & $\mathrm{C} 212$ & $105.9(4)$ & C19 3 & $\mathrm{C} 203$ & $\mathrm{C} 213$ & $105.0(4)$ \\
\hline O3_2 & C21_2 & $\mathrm{N} 2 \_2$ & 127 & O3_3 & C21_3 & N2_3 & $126.6(5)$ \\
\hline O3_2 & C21_2 & C20_2 & 127. & O3_3 & C21_3 & C20_3 & $127.0(5)$ \\
\hline N2_2 & C21_2 & C20_2 & $104.8(4)$ & N2_3 & C21_3 & $\mathrm{C} 20 \_3$ & $106.4(4)$ \\
\hline $1 \_5$ & N1_5 & C9_5 & $126.0(4)$ & $\mathrm{C} 1 \_4$ & N1_4 & C9_4 & $126.4(4)$ \\
\hline C8_5 & N1_5 & C1_5 & 112. & C8_4 & N1_4 & C1_4 & $112.0(4)$ \\
\hline C8_5 & N1_5 & C9_5 & 122 & 4 & N1_4 & C9_4 & $5(4)$ \\
\hline C13_5 & N2_5 & C10_5 & $126.3(4)$ & C13_4 & $\mathrm{N} 2 \_4$ & C10_4 & $124.7(4)$ \\
\hline C21_5 & N2_5 & C10_5 & $122.8(4)$ & C21_4 & N2_4 & C10_4 & $125.1(4)$ \\
\hline C21_5 & N2_5 & C13_5 & 110 & 21_4 & N2_4 & C13_4 & $.3(3)$ \\
\hline O1_5 & C1_5 & N1_5 & $125.5(5)$ & O1_4 & C1_4 & N1_4 & $125.1(4)$ \\
\hline O1_5 & $\mathrm{C} 1 \_5$ & C2_5 & $129.0(5)$ & O1_4 & C1_4 & C2_4 & $129.7(4)$ \\
\hline N1_5 & $\mathrm{C} 1 \_5$ & C2_5 & $105.6(4)$ & N1_4 & C1_4 & C2_4 & $105.2(3)$ \\
\hline C3_5 & $\mathrm{C} 2 \_5$ & C1_5 & 130 & C3_4 & $\mathrm{C} 2 \_4$ & C1_4 & $130.4(4)$ \\
\hline C3_5 & $\mathrm{C} 2 \_5$ & C7_5 & $121.7(4)$ & C3_4 & C2_4 & C7_4 & $121.6(4)$ \\
\hline C7_5 & $\mathrm{C} 2 \_5$ & C1_5 & $107.4(4)$ & C7_4 & C2 4 & C1_4 & $108.0(4)$ \\
\hline $\mathrm{C} 2 \_5$ & C3_5 & C4_5 & $117.3(4)$ & $\mathrm{C} 2 \_4$ & C3_4 & C4_4 & $117.4(4)$ \\
\hline C5_5 & C4_5 & C3_5 & 120 & $5 \_4$ & C4_4 & C3_4 & $120.9(4)$ \\
\hline C6_5 & C5_5 & C4_5 & $121.4(5)$ & C4_4 & C5_4 & C6_4 & $121.3(4)$ \\
\hline C7_5 & C6_5 & C5_5 & $118.1(5)$ & C7 4 & C6_4 & C5 4 & $117.1(4)$ \\
\hline $\mathrm{C} 2 \_5$ & C7_5 & C8_5 & $108.7(4)$ & $\mathrm{C} 2 \_4$ & C7_4 & C6_4 & $121.6(4)$ \\
\hline C6_5 & C7_5 & $\mathrm{C} 2 \_5$ & $120.8(5)$ & $\mathrm{C} 2 \_4$ & C7_4 & C8_4 & $108.4(4)$ \\
\hline C6_5 & C7_5 & C8_5 & $130.6(5)$ & C6_ 4 & C7_4 & C8_4 & $129.9(4)$ \\
\hline O2_5 & C8_5 & N1_5 & $123.8(4)$ & $\mathrm{O} 2 \_4$ & C8 4 & N1_4 & $123.9(4)$ \\
\hline $\mathrm{O} 2 \_5$ & C8_5 & C7_5 & $129.9(4)$ & $\mathrm{O} 2 \_4$ & C8_4 & C7_4 & $129.8(4)$ \\
\hline N1_5 & C8_5 & C7_5 & $106.3(4)$ & N1_4 & C8_4 & C7_4 & $106.3(4)$ \\
\hline N1_5 & C9_5 & C10_5 & $110.7(4)$ & N1_4 & C9_4 & C10_4 & $108.9(4)$ \\
\hline N2_5 & C10_5 & C9 5 & $110.7(4)$ & $\mathrm{N} 24$ & $\mathrm{C} 10 \_4$ & C9 4 & 111.1(3) \\
\hline N2_5 & C10_5 & C11_5 & $110.8(3)$ & N2_4 & C10_4 & C11_4 & 111.1(3) \\
\hline C11_5 & C10_5 & C9_5 & $115.2(4)$ & C11_4 & C10_4 & C9_4 & $114.7(4)$ \\
\hline C12_5 & C11_5 & C10_5 & $126.2(4)$ & C12_4 & C11_4 & C10_4 & $127.1(4)$ \\
\hline C14_5 & C13_5 & N2_5 & $128.6(4)$ & C14_4 & C13_4 & $\mathrm{N} 2 \_4$ & $127.8(4)$ \\
\hline C14_5 & C13_5 & C19_5 & $120.8(4)$ & C14_4 & C13_4 & C19_4 & $121.7(4)$ \\
\hline C19_5 & C13 5 & N2 5 & $110.6(4)$ & $\mathrm{C} 194$ & $\mathrm{C} 13 \_4$ & $\mathrm{~N} 24$ & $110.5(4)$ \\
\hline
\end{tabular}




\begin{tabular}{|c|c|c|c|c|c|c|c|}
\hline $13 \_5$ & C14_5 & C15_5 & $117.4(5)$ & $\mathrm{C} 13 \_4$ & C14_4 & $\mathrm{C} 15 \_4$ & 116 \\
\hline 165 & $\mathrm{C} 155$ & C14 5 & $123.2(5)$ & $\mathrm{C} 164$ & $\mathrm{C} 154$ & $\mathrm{C} 144$ & $123.3(4)$ \\
\hline $15 \_5$ & C16_5 & C17_5 & $121.6(5)$ & C15_4 & C16_4 & C17_4 & $120.9(4)$ \\
\hline $15 \_5$ & C16_5 & C18_5 & $118.5(4)$ & C15_4 & C16_4 & C18_4 & $119.4(4)$ \\
\hline C18_5 & C16_5 & C17_5 & $119.8(5)$ & C18_4 & C16_4 & C17_4 & $119.7(4)$ \\
\hline $19 \_5$ & C18_5 & C16_5 & $119.0(4)$ & C19_4 & C18_4 & C16_4 & $118.5(4)$ \\
\hline C13_5 & C19_5 & C20_5 & $107.2(4)$ & $\mathrm{C} 13 \_4$ & C19_4 & $\mathrm{C} 20 \_4$ & $107.7(4)$ \\
\hline C18_5 & C19_5 & C13_5 & 121.1(4) & C18_4 & C19_4 & C13_4 & $120.9(4)$ \\
\hline C18_5 & C19_5 & C20_5 & $131.8(4)$ & C18_4 & C19_4 & C20_4 & $131.4(4)$ \\
\hline O4_5 & C20_5 & C19_5 & $130.8(5)$ & O4_4 & C20_4 & C19_4 & $130.9(4)$ \\
\hline O4_5 & C20_5 & C21_5 & $123.1(4)$ & $\mathrm{O} 4 \_4$ & $\mathrm{C} 20 \_4$ & C21_4 & $124.0(4)$ \\
\hline C19_5 & C20_5 & C21_5 & 106.1(4) & C19_4 & C20_4 & C21_4 & $105.1(4)$ \\
\hline O3_5 & C21_5 & N2_5 & $127.1(5)$ & O3_4 & C21_4 & N2_4 & $127.6(4)$ \\
\hline O3_5 & C21_5 & C20_5 & $127.6(4)$ & O3_4 & C21_4 & C20_4 & $126.1(4)$ \\
\hline N2_5 & C21_5 & C20_5 & $105.3(4)$ & $\mathrm{N} 2$ & C21_4 & $\mathrm{C} 20 \_4$ & $106.3(4)$ \\
\hline
\end{tabular}

Table S6. Torsion Angles for $\mathbf{9 m j}$.

\begin{tabular}{|c|c|c|c|c|c|c|c|c|c|}
\hline A & B & $\mathrm{C}$ & D & ngle $/^{\circ}$ & $\mathbf{A}$ & B & C & D & Angle/ \\
\hline 1_1 & 1_1 & $2 \_1$ & $3 \_1$ & & $1 \_6$ & 16 & 6 & $3 \_6$ & \\
\hline- & 1 & 1 & & & 6 & 6 & 6 & 7 -6 & \\
\hline 4_1 & 20_1 & 21_1 & 3_1 & 0 & 4_6 & $20 \_6$ & 21_6 & 33 & $2(7)$ \\
\hline 41 & 201 & 211 & 21 & & 46 & 206 & 216 & J 6 & \\
\hline-1 & $1 \_1$ & $2 \_1$ & 3 & & $1 \_6$ & 16 & 2 & $3 \_6$ & \\
\hline 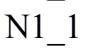 & $1 \_1$ & $\mathrm{C} 2 \_1$ & 7 -1 & & 6 & 6 & 266 & $7 \_6$ & \\
\hline $1 \_1$ & $9 \_1$ & C10_1 & $2 \_1$ & & 6 & 9 & $0 \_6$ & 2 & \\
\hline 1 & 9 & C10_1 & $11 \_1$ & & L_6 & $9 \_6$ & $0 \_6$ & $11 \_6$ & \\
\hline 1 & $0 \_1$ & 1 & & & & $0 \_6$ & & 266 & \\
\hline 2 & & $14 \_1$ & 1 & & 6 & 3 & $4 \_6$ & $5 \_6$ & \\
\hline $2 \_1$ & 1 & $19 \_1$ & 1 & & & 6 & $9 \_6$ & $8 \_6$ & \\
\hline 2 & 13_1 & C19_1 & 0_1 & & 266 & $3 \_6$ & 9_6 & $0 \_6$ & \\
\hline 11 & 1 & 1 & & & 6 & 6 & 6 & 26 & \\
\hline 1_1 & & C8_1 & & & 6 & 16 & 6 & 7_6 & \\
\hline C1_1 & N1_1 & C9_1 & $10 \_1$ & & 6 & 6 & 6 & $0 \_6$ & \\
\hline 1_1 & C2_1 & C3_1 & -1 & & 6 & - 6 & 6 & 4_6 & \\
\hline $1 \_1$ & 1 & 1 & & & 6 & 6 & 6 & 66 & \\
\hline 1_1 & C2_1 & C7_1 & & & & -6 & 7_6 & 6 & \\
\hline C2 21 & C3_1 & C4_1 & C5_1 & & $2 \_6$ & $3 \_6$ & $4 \_6$ & $5 \_6$ & 0 . \\
\hline $\mathrm{C} 2-1$ & C7 7 & $\mathrm{C} 8{ }_{-}^{-} 1$ & 2 & & $2 \_6$ & 7_6 & 8 & $2 \_6$ & \\
\hline C2_1 & -1 & C8_1 & _1 & 1. & - 6 & 7_6 & $8 \_6$ & 1_6 & \\
\hline 3_1 & C2_1 & C7_1 & C6_1 & & 6 & 2 & 7_6 & C6_6 & \\
\hline 3_1 & C2_1 & C7_1 & 1 & & 3 & 2 & 7_6 & $8 \_6$ & \\
\hline 3_1 & 4_1 & C5_1 & 1 & & 6 & $4 \_6$ & 5 & $6 \_6$ & \\
\hline C4_1 & C5_1 & C6_1 & C7_1 & & 4_6 & 5_6 & $6 \_6$ & 7 7_6 & \\
\hline C5_1 & C6_1 & C7_1 & & & 6 & $6 \_6$ & 7 7_6 & C2_6 & \\
\hline & C6_1 & C7_1 & & & 6 & 6 & 7_6 & $8 \_6$ & \\
\hline C6_1 & C7_1 & C8_1 & O2_1 & & C6_6 & 776 & C8_6 & O2_6 & \\
\hline C6_1 & C7_1 & C8_1 & N1_1 & & C6_6 & 7 6_6 & $8 \_6$ & N1_6 & $179.0(5)$ \\
\hline & & C3_1 & & & 6 & 6 & C3_6 & C4_6 & \\
\hline $8 \quad 1$ & {$\left[\begin{array}{lll}1 & 1\end{array}\right.$} & $\mathrm{C} 1 \_1$ & O1_1 & 17 & C8 6 & N1_6 & C1_6 & O1_6 & $178.5(5)$ \\
\hline
\end{tabular}




\begin{tabular}{|c|c|c|c|c|c|c|c|c|c|}
\hline-1 & -1 & 1 & $2-1$ & & C8_6 & N1_6 & C1_6 & & \\
\hline $8 \_1$ & $1 \_1$ & 9 & $10 \_1$ & & $8 \_6$ & 1_6 & C9_6 & C10_6 & $-65.4(5)$ \\
\hline 1 & $1 \_1$ & 1 & 1 & .1(9) & $9 \_6$ & $1 \_6$ & $1 \_6$ & O1_6 & (10) \\
\hline 9_1 & 1_1 & 1 & 2 & $-179.7(5$ & 99 & 116 & C1_6 & $\mathrm{C} 2 \_6$ & 0.91 \\
\hline 91 & 11 & 81 & 21 & 10 & 96 & 16 & 86 & 26 & \\
\hline 91 & $1 \_1$ & 8 & 7 & & 96 & $1 \_6$ & $8 \_6$ & 766 & \\
\hline $9 \_1$ & $10 \_1$ & 11_1 & $1 \overline{2} \_1$ & $1.3(8)$ & 9 & $10 \_6$ & $11 \_6$ & $1 \overline{2} 6$ & $4.0(4)$ \\
\hline 101 & $2 \_1$ & $\mathrm{C} 13 \_1$ & $14 \_1$ & 0.5 & 106 & 26 & C13 6 & 6 & 7) \\
\hline $10 \_1$ & $2 \_1$ & C13_1 & 19_1 & -170 & $0 \_6$ & $2-6$ & $13 \_6$ & C19_6 & \\
\hline 01 & 21 & 11 & 31 & & 06 & 26 & $\mathrm{C} 216$ & 36 & \\
\hline 10_1 & $2 \_1$ & 1_1 & 20_1 & 72. & 6 & N2_6 & C21_6 & $20 \_6$ & -1 \\
\hline 131 & 21 & $0 \_1$ & 91 & -77.6 & 36 & 26 & C10_6 & C9 6 & $-72.1(5)$ \\
\hline $13 \_1$ & $2 \_1$ & $10 \_1$ & $11 \_1$ & $51.9(7)$ & $3 \_6$ & $2 \_6$ & $10 \_6$ & $11 \_6$ & 7(5) \\
\hline 13 & $2 \_1$ & & 1 & & 6 & 6 & 16 & $\overline{6}$ & 2(4) \\
\hline 13_1 & $2 \_1$ & -1 & 20_1 & $8(6)$ & _6 & I2_6 & C21_6 & $0 \_6$ & \\
\hline 131 & 141 & & 1 & -2.1 & & 46 & & $\mathrm{C} 166$ & 8) \\
\hline 3_1 & 19_1 & $0 \_1$ & 1 & - & 6 & 19_6 & 0_6 & 6 & \\
\hline 3_1 & 19_1 & __1 & 1 & ) & _6 & 9_6 & 0_6 & $1 \_6$ & \\
\hline 14_1 & 13_1 & & & & & $3 \_6$ & C19_6 & & \\
\hline 141 & 131 & 1 & 1 & 177. & & 6 & 6 & 6 & (4) \\
\hline 4_1 & 5_1 & 1 & 1 & & 6 & 6 & 6 & 6 & \\
\hline 4_1 & _ 1 & 1 & & & & 6 & 6 & 6 & \\
\hline 5_1 & 16_1 & & & & & & & & \\
\hline 61 & 181 & & & $0.1(7)$ & 6 & 6 & 6 & 6 & \\
\hline 6_1 & 18_1 & 1 & & & 6 & 6 & 6 & 6 & \\
\hline _1 & -1 & & & & & & 6 & 6 & \\
\hline 8_1 & 19_1 & & & & & & 6 & & \\
\hline 81 & 191 & $\mathrm{C} 2$ & & -1 & 6 & & 6 & & 1 \\
\hline $9 \_1$ & 13_1 & 1 & & 3 & 6 & 6 & 6 & 6 & \\
\hline-1 & 1 & & $\mathrm{O} 3$ & & & & _6 & & \\
\hline 19_1 & C20_1 & C21_1 & 1 & -1 & 6 & 6 & C21_6 & 6 & \\
\hline 21_1 & $2 \_1$ & C10_1 & 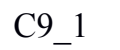 & & -6 & 6 & $0 \_6$ & 6 & T) \\
\hline $1 \_1$ & $2 \_1$ & & & & & & 6 & & \\
\hline 1 1_1 & 2_1 & & & & & & & & -1 \\
\hline C21_1 & $2 \_1$ & C13_1 & C19_1 & $0.1(6)$ & 6 & 6 & C13_6 & C19_6 & \\
\hline $1 \_2$ & 1 2 & $C_{2} 2$ & $C_{3} 2$ & & 3 & 3 & 3 & $3 \_3$ & 8) \\
\hline O1_2 & $\mathrm{C} 1 \_2$ & $\mathrm{C} 2 \_2$ & $e^{2}$ & & 3 & 3 & 3 & 3 & 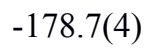 \\
\hline 4 4_2 & C20_2 & 21_2 & O3_2 & $0.5(9)$ & & & C21_3 & O3_3 & (8) \\
\hline O4_2 & C20_2 & C21_2 & J2_2 & 1702 & +3 & C20_3 & C21_3 & N2_3 & \\
\hline $1 \_2$ & 112 & $\mathrm{C} 2{ }_{2}$ & 2 & -1 & 3 & 3 & $2 \_3$ & C $3 \_3$ & 1 \\
\hline N1_2 & $\mathrm{C} 1 \_2$ & $\mathrm{C} 2 \_2$ & C7_2 & 1 & 3 & 3 & 23 & 73 & 5) \\
\hline N1_2 & C9_2 & C10_2 & N2_2 & $-67.7(5$ & $1 \_3$ & C9_3 & C10_3 & $\mathrm{N} 2 \_3$ & $-70.1(4)$ \\
\hline I1_2 & C9 2 & C10_2 & 1112 & $=2$ & & C9 3 & C10_3 & C11_3 & $6(3)$ \\
\hline N2_2 & C10_2 & C11_2 & 2 & -1 & & $10 \_3$ & C11_3 & $\mathrm{C} 12 \_3$ & (7) \\
\hline N2_2 & C13_2 & C14_2 & C15_2 & -179 & $2 \_3$ & C13_3 & C14_3 & $15 \_3$ & 175 \\
\hline N2_2 & C13_2 & C19_2 & $18 \_2$ & $179.0(5)$ & $2 \_3$ & C13_3 & C19_3 & C18_3 & $176.6(4)$ \\
\hline N2_2 & C13_2 & C19_2 & C20_2 & & & $3 \_3$ & C19_3 & 3 & \\
\hline C1_2 & N1_2 & C8_2 & O2_2 & . & $1 \_3$ & 1_3 & C8_3 & O2_3 & $177.5(4)$ \\
\hline C1_2 & N1_2 & C8_2 & C7_2 & $0.2(6)$ & C1_3 & N1_3 & C8_3 & C7_3 & $-2.8(5)$ \\
\hline C1_2 & N1_2 & C9_2 & C10_2 & $104.2(6)$ & C1_3 & N1_3 & C9_3 & C10_3 & $117.7(4)$ \\
\hline
\end{tabular}




\begin{tabular}{|c|c|c|c|c|c|c|c|c|c|}
\hline 12 & $2 \_2$ & C3_2 & 2 & & 3 & C2_3 & C3_3 & C4_3 & \\
\hline C1_2 & $2-2$ & 7 2 2 & $6 \_2$ & 177 & 1 1_3 & $\mathrm{C} 2{ }_{-}^{-} 3$ & C7_3 & C6_3 & $9.2(4)$ \\
\hline $1 \_2$ & 22 & C7_2 & $8 \_2$ & $1.7(6)$ & $1 \_3$ & C2_3 & 7 -3 & C8_3 & $.6(5)$ \\
\hline 22 & 32 & C4 2 & 52 & .8 & 23 & 33 & 43 & 53 & 587 \\
\hline 2 & $7 \_2$ & $8 \_2$ & $2 \_2$ & & $2 \_3$ & 7 -3 & $8 \_3$ & $2 \_3$ & \\
\hline 2 & 7_2 & $8 \_2$ & $1 \_2$ & $1.0(6)$ & $2 \_3$ & C7_3 & $8 \_3$ & 11_3 & $3(5)$ \\
\hline $3 \_2$ & 22 & C7_2 & $6 \_2$ & $-1.3(9)$ & 3_3 & C2_3 & 7_3 & 663 & $0.5(7)$ \\
\hline 32 & 22 & C7 2 & 82 & 179 & 33 & $\mathrm{C} 23$ & 73 & 83 & 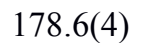 \\
\hline 32 & $4 \_2$ & $5 \_2$ & 6_2 & 0) & $3 \_3$ & 4 & $5 \_3$ & $6 \_3$ & (7) \\
\hline $4 \_2$ & $5 \_2$ & C6_2 & $7 \_2$ & 9) & 3 & 553 & $6 \_3$ & $7 \_3$ & (7) \\
\hline $5 \_2$ & $6 \_2$ & C7_2 & $2 \_2$ & 3) & $5 \_3$ & C6_3 & $7 \_3$ & $2 \_3$ & (7) \\
\hline $5 \_2$ & $6 \_2$ & C7_2 & $8 \_2$ & 179 & $5 \_3$ & C6_3 & 773 & $8 \_3$ & \\
\hline $6 \_2$ & 7_2 & $8 \_2$ & 2 & - & 5 & 7_3 & 8_3 & 2 23 & \\
\hline $6 \_2$ & $7 \_2$ & C8_2 & $1 \_2$ & -17 & $6 \_3$ & C7_3 & 8_3 & $1 \_3$ & - \\
\hline 7_2 & $2 \_2$ & C3_2 & $4 \_2$ & $1.5(8)$ & $7 \_3$ & $\mathrm{C} 2 \_3$ & 3_3 & C4_3 & 6) \\
\hline 2 & 1_2 & C1_2 & $1 \_2$ & 78 & $8 \_3$ & J1_3 & $1 \_3$ & D1_3 & - \\
\hline 2 & $1 \_2$ & $1 \_2$ & 2 & . & 3 & $1 \_3$ & 3 & $2 \_3$ & \\
\hline $8 \_2$ & J1_2 & C9_2 & 10_2 & -73. & 8_3 & N1_3 & $9 \_3$ & $10 \_3$ & $2(5)$ \\
\hline $9 \_2$ & N1_2 & C1_2 & O1_2 & $1.2(9)$ & $9 \_3$ & N1_3 & 1 3 & O1_3 & $-0.7(7)$ \\
\hline 2 & 1_2 & $1 \_2$ & 2 & -178 & $9 \_3$ & $1 \_3$ & $1 \_3$ & $2 \_3$ & $179.6(4)$ \\
\hline 2 & $1 \_2$ & 8 & 2 & -3 & 9 & $1 \_3$ & 3 & $2 \_3$ & \\
\hline 9_ 2 & 112 & $8 \_2$ & 7_2 & & 933 & 3 & 8_3 & C7_3 & \\
\hline $9 \_2$ & $10 \_2$ & C11_2 & $12 \_2$ & $0.7(6)$ & C9 3 & $\mathrm{C} 10 \_3$ & C11_3 & C12_3 & $9(5)$ \\
\hline $0 \_2$ & 2_2 & C13_2 & $4 \_2$ & 2.2 & $10 \_3$ & N2_3 & $13 \_3$ & $14 \_3$ & 7 \\
\hline $0 \_2$ & 22 & $13 \_2$ & $9 \_2$ & -17 & $0 \_3$ & J2_3 & $13 \_3$ & C19_3 & \\
\hline $0 \_2$ & $2 \_2$ & $21 \_2$ & $3 \_2$ & $-2.7(9)$ & $10 \_3$ & N2_3 & $21 \_3$ & O3_3 & \\
\hline $0 \_2$ & 22 & C21_2 & $20 \_2$ & 178. & $10 \_3$ & N2_3 & $21 \_3$ & C20_3 & 1 \\
\hline 3_2 & $2 \_2$ & $10 \_2$ & $9 \_2$ & $-68 .($ & $13 \_3$ & N2_3 & $10 \_3$ & $9 \_3$ & 5) \\
\hline $3 \_2$ & 2 & $0 \_2$ & $1 \_2$ & 60 & 3 & $\mathrm{~N} 2 \_3$ & 0_3 & $1 \_3$ & 6) \\
\hline 3_2 & $2 \_2$ & 21_2 & & & & N2_3 & $1 \_3$ & 3 & \\
\hline 322 & J2_2 & C21_2 & $20 \_2$ & $0.9(6)$ & $13 \_3$ & N2_3 & $21 \_3$ & C20_3 & 0 \\
\hline 3_2 & 14_2 & $15 \_2$ & $6 \_2$ & -0 . & 3 & $14 \_3$ & $5 \_3$ & 53 & -( \\
\hline $3 \_2$ & $19 \_2$ & $20 \_2$ & +2 & -17 & 3 & $19 \_3$ & $20 \_3$ & $4 \_3$ & - \\
\hline 3_2 & $19 \_2$ & $20 \_2$ & 21_2 & & & C19_3 & 0_3 & $1 \_3$ & \\
\hline $4 \_2$ & $13 \_2$ & 192 & $18 \_2$ & -1.0 & $14 \_3$ & $\mathrm{C} 13 \_3$ & $19 \_3$ & C18_3 & 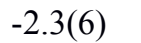 \\
\hline $4 \_2$ & $13 \_2$ & $19 \_2$ & 20_2 & 17 & $4 \_3$ & C13_3 & $9 \_3$ & C20_3 & 1 \\
\hline $4 \_2$ & $15 \_2$ & 5 & 72 & -179 & t_3 & 3 & 3 & 73 & $5(4)$ \\
\hline $4 \_2$ & C15_2 & C16_2 & C18_2 & $1.0(7)$ & $14 \_3$ & C15_3 & $16 \_3$ & C18_3 & $-2.2(7)$ \\
\hline $5 \_2$ & 16_2 & $18 \_2$ & $9 \_2$ & -1.2 & $5 \_3$ & C16_3 & $8 \_3$ & C19_3 & 6) \\
\hline $6 \_2$ & $18 \_2$ & 92 & 32 & 1.2 & 3 & 3 & 3 & C13_3 & -0 \\
\hline $6 \_2$ & $18 \_2$ & C19_2 & C20_2 & -178 & $16 \_3$ & C18_3 & C19_3 & C20_3 & $178.9(5)$ \\
\hline 17_2 & C16_2 & C18_2 & $19 \_2$ & $1788(4)$ & $17 \_3$ & C16_3 & C18_3 & C19_3 & -179. \\
\hline 82 & 192 & $20 \_2$ & 2 & 0 & 33 & $\mathrm{C} 193$ & 3 & O4_3 & 5. \\
\hline C18_2 & C19_2 & C20_2 & 21_2 & -178.6( & $18 \_3$ & C19_3 & C20_3 & C21_3 & -176.4 \\
\hline C19_2 & C13_2 & C14_2 & C15_2 & $0.7(7)$ & 19 -3 & C13_3 & C14_3 & C15_3 & $2.9(6)$ \\
\hline 9_2 & C20_2 & C21_2 & 3_2 & 1800 & $19 \_3$ & C20_3 & 3 & O3_3 & $178.1(5$ \\
\hline $9 \_2$ & C20_2 & C21_2 & N2_2 & $-1.3(6)$ & 19 3 & C20_3 & C21_3 & N2_3 & $-2.3(5)$ \\
\hline C21_2 & N2_2 & C10_2 & C9_2 & $114.5(5$ & C21_3 & N2_3 & C10_3 & C9_3 & $111.5(4)$ \\
\hline $21 \_2$ & N2_2 & C10_2 & $11 \_2$ & $-116.9(5)$ & $21 \_3$ & N2_3 & C10_3 & C11_3 & $-120.4(4)$ \\
\hline C21_2 & N2_2 & C13_2 & C14_2 & $180.0(5)$ & C21_3 & N2_3 & C13_3 & C14_3 & $-179.6(4)$ \\
\hline
\end{tabular}




\begin{tabular}{|c|c|c|c|c|c|c|c|c|c|}
\hline $21 \_2$ & $2 \_2$ & $13 \_2$ & C19_2 & $0.0(6)$ & C21_3 & N2_3 & C13_3 & C19_3 & $1.5(5)$ \\
\hline $01 \_\overline{5}$ & $\mathrm{C} 1 \_5$ & $\mathrm{C} 2 \_\overline{5}$ & C3_5 & $1.6(9)$ & O1_ 4 & $\mathrm{C} 1 \_4$ & $\mathrm{C} 2 \_\overline{4}$ & C3_ 4 & $2.5(8)$ \\
\hline $1 \_5$ & C1_5 & $\mathrm{C} 2 \_5$ & C7_5 & $-176.8(5)$ & $1 \_4$ & $\mathrm{C} 1 \_4$ & $\mathrm{C} 2 \_4$ & C7_4 & 1750 \\
\hline 4 -5 & C20_5 & $21 \_5$ & O3_5 & $-1.0(7)$ & 4 & $\mathrm{C} 2 \mathrm{C}_{4} 4$ & C21_4 & O3_4 & $2.7(7)$ \\
\hline $4+5$ & $\mathrm{C} 20 \_5$ & $21 \_5$ & N2_5 & $178.5(4)$ & 4 & $\mathrm{C} 2 \mathrm{C}_{4} 4$ & C21_4 & $\mathrm{N} 2-4$ & $-178.7(4)$ \\
\hline $1 \_5$ & C1_ 5 & $2 \_\overline{5}$ & C3_5 & -179 & $1 \_4$ & $\mathrm{C} 1 \_\overline{4}$ & $\mathrm{C} 2 \_\overline{4}$ & C3_4 & -178 \\
\hline 15 & C1 5 & $\mathrm{C} 25$ & C7 5 & $2.4(5)$ & 14 & $\mathrm{C} 14$ & $\mathrm{C} 24$ & C7 4 & $3.6(5)$ \\
\hline $1 \_5$ & C9_5 & C10_5 & N2_5 & -67.5 & $1 \_4$ & C9_4 & C10_4 & N2_4 & $-69.1(4)$ \\
\hline 15 & C9 5 & C10 5 & $\mathrm{C} 11 \_5$ & 165. & 4 & C9 4 & $\mathrm{C} 10 \_4$ & 114 & $163.9(3)$ \\
\hline $2 \_5$ & C10_5 & C11_5 & $\mathrm{C} 12 \_5$ & -129 & 24 & C10_4 & C11_4 & C12_4 & -132 \\
\hline 25 & $\mathrm{C} 135$ & 145 & $\mathrm{C} 155$ & -178 & 4 & 134 & $\mathrm{C} 144$ & 54 & -179 \\
\hline $2 \_5$ & C13_5 & $19 \_5$ & C18_5 & 178. & 4 & C13_4 & C19_4 & C18_4 & $179.6(4)$ \\
\hline 25 & $\mathrm{C} 13 \_5$ & C19_5 & $\mathrm{C} 20 \_5$ & $-1.3(5)$ & 24 & $\mathrm{C} 134$ & $\mathrm{C} 19 \_4$ & $\mathrm{C} 2 \mathrm{C}_{4}$ & $-1.3(5)$ \\
\hline $1 \leq 5$ & N1_5 & C8_5 & O2_5 & 178 & $1 \_4$ & J1_4 & C8_4 & $\mathrm{O} 2 \_4$ & $179.5(4)$ \\
\hline $1 \_5$ & N1_5 & C8_5 & C7_5 & -1.3 & 4 & $1 \_4$ & C8_4 & 4 & $0.0(5)$ \\
\hline $1 \_5$ & N1_5 & C9_5 & C10_5 & 108 & $1 \_4$ & N1_4 & C9_4 & $0 \_4$ & $102.7(5)$ \\
\hline 5 & $\mathrm{C} 25$ & C3 5 & C4 5 & -176 & 14 & $\mathrm{C} 24$ & C3 4 & C4 4 & $-178.1(4)$ \\
\hline $1 \leq 5$ & $\mathrm{C} 2-5$ & C7_5 & C6_5 & 177 & $1 \_4$ & C2_4 & $7 \_4$ & 5 & 177 \\
\hline $1 \_5$ & $\mathrm{C} 2 \_5$ & C7_5 & C8_5 & -3.3 & 4 & $2 \_4$ & 74 & 4 & -3 \\
\hline $2 \_5$ & C3_5 & C4_5 & C5_5 & -1.1 & 4 & C3_4 & C4_4 & & $1.0(7)$ \\
\hline 25 & C7 5 & C8 5 & O2 5 & -176 & 4 & C7 4 & C8 4 & $\mathrm{O} 24$ & -177.1 \\
\hline $2 \_5$ & C7_5 & C8_5 & N1_5 & 2.9 & -4 & $7 \_4$ & 8_4 & L_ 4 & $2.4(5)$ \\
\hline 3 & $\mathrm{C} 2 \_5$ & C7_5 & C6_5 & -1 . & 4 & $\mathrm{C} 2 \_4$ & C7_4 & 5 & $-1.3(7)$ \\
\hline 3 & $\mathrm{C} 2 \_5$ & C7_5 & C8_5 & & & $\mathrm{C} 2 \_4$ & C7_4 & 4 & $177.8(4)$ \\
\hline 35 & C4 5 & C5 5 & C6 5 & 0.3 & 4 & C4 4 & C5 4 & 4 & -0 \\
\hline 45 & C5_5 & C6_5 & C7_5 & 0 & & 5 & C6_4 & 4 & -0 \\
\hline 5 & C6_5 & C7_5 & $\mathrm{C} 2 \_5$ & 0.3 & & C6_4 & C7_4 & 4 & $1.4(7)$ \\
\hline 5 & C6_5 & C7_5 & C8_5 & -17 & & C6_4 & C7_4 & 4 & \\
\hline 65 & C7 5 & C8 5 & O2 5 & 2.6 & 4 & C7 4 & C8 4 & $\mathrm{O} 24$ & 2.0 \\
\hline $6 \_5$ & C7_5 & C8_5 & -5 & -17 & 4 & 4 & C8_4 & -4 & -17 \\
\hline $7 \_5$ & $\mathrm{C} 2 \_5$ & C3_5 & C4_5 & 1.4 & 4 & $\mathrm{C} 2 \_4$ & C3_4 & C4_4 & $0.1(7)$ \\
\hline $8 \_5$ & N1_5 & C1_5 & O1_5 & 178 & 8_4 & N1_4 & C1_4 & O1_4 & $177.3(4)$ \\
\hline $8 \_5$ & N1_5 & C1_5 & $\mathrm{C} 2 \_5$ & -0.6 & $8 \_4$ & N1_4 & C1_4 & $\mathrm{C} 2 \_4$ & $-2.2(5)$ \\
\hline 3 & 5 & C9_5 & C10_5 & -70 & 4 & 1_4 & C9_4 & C10_4 & $-75.4(5)$ \\
\hline $9 \_5$ & N1_5 & C1_5 & O1_5 & -1.0 & $9 \_4$ & N1_4 & C1_4 & O1_4 & $-0.9(7)$ \\
\hline C9 5 & N1_5 & C1_5 & C2_ 5 & 179 & 94 & N1_4 & C1_4 & C2 4 & $179.5(4)$ \\
\hline 9 & N1_5 & C8_5 & $\mathrm{O} 2 \_5$ & -2.2 & $9 \_4$ & N1_4 & C8_4 & $\mathrm{O} 2 \_4$ & $-2.1(7)$ \\
\hline C9_5 & N1_5 & C8_5 & C7_5 & 178 & $9 \_4$ & N1_4 & C8_4 & C7_4 & $178.4(4)$ \\
\hline $9 \_5$ & C10_5 & C11_5 & $\mathrm{C} 12 \_5$ & -3.0 & $9 \_4$ & $\mathrm{C} 10_{4} 4$ & C11_4 & $\mathrm{C} 12 \_4$ & $-5.0(6)$ \\
\hline $10 \_5$ & N2_5 & C13_5 & C14_5 & $5.9{ }^{\circ}$ & 104 & N2_4 & $\mathrm{C} 13 \_4$ & $\mathrm{C} 14 \_4$ & $4.0(6)$ \\
\hline $10 \_5$ & N2_5 & C13_5 & C19_5 & -174 & $10 \_4$ & $\mathrm{~N} 2 \_4$ & C13_4 & C19_4 & -176.3 \\
\hline $10 \_5$ & $\mathrm{~N} 2 \_5$ & C21_5 & O3_5 & -5.1 & 4 & $\mathrm{~N} 2 \_4$ & C21_4 & O3_4 & $-5.4(7)$ \\
\hline $10 \_5$ & N2_5 & C21_5 & C20_5 & 175.4 & $10 \_4$ & N2_4 & C21_4 & C20_4 & $176.0(3)$ \\
\hline $13 \_5$ & N2_ 5 & C10_5 & C9 5 & -73. & 134 & N2_4 & $\mathrm{C} 10 \_4$ & C9 4 & $-73.0(5)$ \\
\hline 135 & N2_5 & C10_5 & C11_5 & 55.7 & 34 & $\mathrm{~N} 2 \_4$ & C10_4 & C11_4 & $56.0(5)$ \\
\hline $13 \_5$ & $\mathrm{~N} 2 \_5$ & C21_5 & O3_5 & $178.9(4)$ & $13 \_4$ & $\mathrm{~N} 2 \_4$ & C21_4 & O3_4 & $175.3(4)$ \\
\hline $13 \_5$ & N2_5 & C21_5 & C20_5 & $-0.6(5)$ & $13 \_4$ & N2_4 & C21_4 & C20_4 & $-3.3(4)$ \\
\hline 5 & C14_5 & $\mathrm{C} 15 \_5$ & $\mathrm{C} 165$ & -0.9 & & $\mathrm{C} 1444$ & $\mathrm{C} 154$ & $\mathrm{C} 1644$ & $0.5(6)$ \\
\hline $13 \_5$ & C19_5 & $\mathrm{C} 20 \_5$ & O4_5 & $-177.7(5$ & C13_4 & $\mathrm{C} 19 \_4$ & $\mathrm{C} 20 \_4$ & O4_ 4 & -179.4 \\
\hline C13 5 & $\mathrm{C} 19 \_5$ & $\mathrm{C} 20 \_5$ & $\mathrm{C} 21 \_5$ & $0.9(5)$ & $\mathrm{C} 13 \_4$ & $\mathrm{C} 194$ & $\mathrm{C} 20 \_4$ & $\mathrm{C} 21 \_4$ & $-0.7(4)$ \\
\hline
\end{tabular}




\begin{tabular}{|c|c|c|c|c|c|c|c|c|c|}
\hline+5 & $13 \_5$ & 19 5 & C18_5 & $-2.1(7)$ & C14_4 & $\mathrm{C} 13 \_4$ & C19_4 & C18_4 & $-0.7(6)$ \\
\hline 145 & C13_5 & C19_5 & $\mathrm{C} 20 \_5$ & $178.2(4)$ & C14_4 & C13_4 & C19_4 & C20_4 & $178.5(4)$ \\
\hline 14 & C15_5 & C16_5 & C17_5 & -178.5 & $14 \_4$ & C15_4 & C16_4 & C17_4 & $-178.5(4)$ \\
\hline $14 \_5$ & C15_5 & C16_5 & C18_5 & $-0.4(8)$ & 14 & C15_4 & $\mathrm{C} 16^{-} 4$ & $\mathrm{C} 18^{-} 4$ & $-1.1(7)$ \\
\hline 5 & C16_5 & C18_5 & C19 5 & $0.4(7)$ & 54 & $\mathrm{C} 16 \_4$ & $\mathrm{C} 18 \_4$ & $\mathrm{C} 19 \_4$ & $0.8(6)$ \\
\hline 165 & C18_5 & C19_5 & C13_5 & $0.8(7)$ & C16_4 & C18_4 & C19_4 & C13_4 & $0.0(6)$ \\
\hline 65 & C18 5 & C19 5 & $\mathrm{C} 205$ & -179 & 64 & $\mathrm{C} 184$ & C19 4 & $\mathrm{C} 204$ & $9(4)$ \\
\hline 17 5 & C16_5 & C18_5 & C19_5 & $178.5(4)$ & C17_4 & C16_4 & C18_4 & C19_4 & $178.2(4)$ \\
\hline $18 \_5$ & C19_5 & $\mathrm{C} 20 \_5$ & O4 5 & $2.7(8)$ & $\mathrm{C} 18 \_4$ & $\mathrm{C} 19 \_4$ & $\mathrm{C} 20 \_4$ & O4_ 4 & $-0.4(8)$ \\
\hline $8 \_5$ & C19_5 & C20_5 & C21_5 & -178.7 & C18_4 & C19_4 & C20_4 & C21_4 & $178.3(4)$ \\
\hline 95 & $\mathrm{C} 135$ & $\mathrm{C} 145$ & C15 5 & $2.1(7)$ & $\mathrm{C} 194$ & $\mathrm{C} 134$ & $\mathrm{C} 144$ & C15 4 & $0.4(6)$ \\
\hline $19-5$ & $\mathrm{C} 20 \_5$ & $\mathrm{C} 21{ }^{-} 5$ & O3_ $\overline{5}$ & $-179.7(4)$ & $\mathrm{C} 19 \_4$ & C20_4 & $\mathrm{C} 21 \_4$ & O3_ 4 & $-176.1(4)$ \\
\hline 195 & $\mathrm{C} 20 \_5$ & C21_5 & $\mathrm{N} 25$ & $-0.2(5)$ & $\mathrm{C} 19 \_4$ & $\mathrm{C} 20 \_4$ & $\mathrm{C} 21 \_4$ & $\mathrm{~N} 24$ & $2.5(4)$ \\
\hline $21 \_5$ & N2_5 & C10_5 & C9_5 & 111.3(4) & C21_4 & N2_4 & C10_4 & C9 4 & $107.8(5)$ \\
\hline $21 \_5$ & $\mathrm{~N} 2 \_5$ & C10_5 & C11_5 & $-119.7(4)$ & C21_4 & N2_4 & C10_4 & C11_4 & $-123.2(4)$ \\
\hline $21 \_5$ & N2_5 & C13_5 & C14_5 & $-178.2(5)$ & C21_4 & N2_4 & C13_4 & C14_4 & $-176.7(4)$ \\
\hline $\mathrm{C} 215$ & N2 5 & $\mathrm{C} 135$ & C19 5 & $1.2(5)$ & $\mathrm{C} 214$ & $\mathrm{~N} 24$ & $\mathrm{C} 134$ & $\mathrm{C} 194$ & $3.0(5)$ \\
\hline
\end{tabular}

Table S7. Hydrogen Atom Coordinates $\left(\AA \times 10^{4}\right)$ and Isotropic Displacement Parameters $\left(\AA^{2} \times 10^{3}\right)$ for 9mj.

\begin{tabular}{|c|c|c|c|c|}
\hline Atom & . & & & \\
\hline H3_1 & 7891.8 & 7430.86 & 4757.03 & 36 \\
\hline $\mathrm{H} 4 \_1$ & 7325.32 & 7861.23 & 4891.82 & 41 \\
\hline H5_1 & 6491.34 & 8144.41 & 3538.05 & 39 \\
\hline H6_1 & 6170.39 & 8002.97 & 1958.31 & 35 \\
\hline H9A_1 & 7658.81 & 6879.7 & 1645.83 & 31 \\
\hline H9B_1 & 7515.2 & 7116.13 & 876.9 & 31 \\
\hline H10_1 & 5753.85 & 7088.2 & 277.53 & 31 \\
\hline H11_1 & 5508.08 & 6681.15 & -734.33 & 37 \\
\hline H12A 1 & 7369.39 & 6864.76 & -282.64 & 34 \\
\hline H12B_1 & 6620.13 & 6670.65 & -1219.39 & 34 \\
\hline H14_1 & 7270.06 & 6406.87 & 1210.68 & 32 \\
\hline H15_1 & 7405.4 & 5948.72 & 1688.34 & 35 \\
\hline H17A_1 & 6833.59 & 5635.02 & 3002.15 & 55 \\
\hline H17B_1 & 6674.28 & 5544.09 & 1947.96 & 55 \\
\hline $\mathrm{H} 17 \mathrm{C} \_1$ & 5745.5 & 5568.26 & 2116.07 & 55 \\
\hline $\mathrm{H} 18 \_-$ & 5091.17 & 6032.64 & 2165.52 & 34 \\
\hline H3_ 2 & 7823.43 & 7387.39 & 9610.9 & 44 \\
\hline $\mathrm{H} 4 \_2$ & 7307.04 & 7809.49 & 9899.25 & 48 \\
\hline $\mathrm{H} 5 \_2$ & 6451.74 & 8118.26 & 8649.06 & 44 \\
\hline H6_2 & 6065.52 & 8020.23 & 7016.94 & 36 \\
\hline H9A__2 & 7444.42 & 6931.02 & 6281.3 & 30 \\
\hline H9B_2 & 7181.78 & 7181.32 & 5531.21 & 30 \\
\hline H10_2 & 5460.45 & 7124.74 & 5088.46 & 28 \\
\hline H11_2 & 5115.39 & 6755.18 & 3948.66 & 29 \\
\hline H12A_2 & 7075.15 & 6865.62 & 4496.27 & 38 \\
\hline H12B_2 & 6255.53 & 6705.39 & 3493.61 & 38 \\
\hline H14_2 & 7177.37 & 6483.62 & 5896.51 & 26 \\
\hline H15_2 & 7663.28 & 6050.96 & 6613.2 & 27 \\
\hline H17A 2 & 7219.18 & 5746.93 & 8285.37 & 43 \\
\hline
\end{tabular}




\begin{tabular}{|c|c|c|c|c|}
\hline H17B_2 & 7617.16 & 5682.65 & 7539.48 & 43 \\
\hline $\mathrm{H} 17 \mathrm{C} \_2$ & 6507.8 & 5604.31 & 7245.25 & 43 \\
\hline H18_2 & 5583.74 & 6066.29 & 7446.75 & 29 \\
\hline H3_5 & 2777.32 & 4031.98 & 790.7 & 30 \\
\hline $\mathrm{H} 4 \_5$ & 2477.36 & 4454.34 & -39.86 & 35 \\
\hline H5_5 & 3772.74 & 4757.8 & 350.5 & 34 \\
\hline H6_5 & 5397.53 & 4649.31 & 1552.55 & 32 \\
\hline H9A_5 & 6130.81 & 3558.74 & 3676.41 & 30 \\
\hline H9B_5 & 6861.69 & 3811.29 & 4192.32 & 30 \\
\hline H10_5 & 7358.2 & 3768.85 & 2954.65 & 27 \\
\hline H11_5 & 8504.22 & 3394.19 & 3755.62 & 29 \\
\hline H12A_5 & 7975.25 & 3520.64 & 5167.63 & 30 \\
\hline H12B_5 & 8974.99 & 3357.82 & 5350.8 & 30 \\
\hline H14_5 & 6581.84 & 3097.77 & 3759.45 & 28 \\
\hline H15_5 & 6000.66 & 2645.59 & 3482.78 & 30 \\
\hline H17A_5 & 5362.51 & 2249.01 & 2505.22 & 47 \\
\hline H17B_5 & 5450.82 & 2228.87 & 1528.83 & 47 \\
\hline $\mathrm{H} 17 \mathrm{C} \_5$ & 4451.68 & 2338.11 & 1459.99 & 47 \\
\hline H18_ 5 & 5244.55 & 2692.76 & 608.22 & 29 \\
\hline H3_6 & 2917.69 & 4146.25 & 5523.89 & 30 \\
\hline H4_6 & 2849.17 & 4593.64 & 4929.99 & 31 \\
\hline H5_6 & 4237.11 & 4873.58 & 5683.6 & 29 \\
\hline H6_6 & 5733.65 & 4721.2 & 7025.39 & 28 \\
\hline H9A_6 & 5778.15 & 3587.49 & 8647.75 & 25 \\
\hline H9B_6 & 6424.54 & 3829.18 & 9374.1 & 25 \\
\hline H10_6 & 7406.97 & 3821.85 & 8554.42 & 24 \\
\hline H11_6 & 7908.77 & 3560.79 & 10171.58 & 29 \\
\hline H12A_6 & 8514 & 3281.1 & 9018.62 & 37 \\
\hline H12B_6 & 8985.65 & 3256.32 & 10222.11 & 37 \\
\hline H14_6 & 6385.66 & 3133.75 & 8866.09 & 26 \\
\hline H15_6 & 5786.86 & 2689.48 & 8390.24 & 29 \\
\hline H17A_6 & 5371.65 & 2308.39 & 7337.38 & 51 \\
\hline H17B_6 & 5545.33 & 2318.11 & 6410.24 & 51 \\
\hline H17C_6 & 4517.87 & 2419.7 & 6294.41 & 51 \\
\hline H18_6 & 5589.37 & 2798.41 & 5730.67 & 31 \\
\hline H3_3 & 3338 & 5792.28 & 5680.04 & 28 \\
\hline $\mathrm{H} 4 \_3$ & 2595.68 & 6216.32 & 4992.85 & 32 \\
\hline H5_3 & 3080.41 & 6466.37 & 4036.77 & 31 \\
\hline H6_3 & 4365.68 & 6308.72 & 3788.83 & 28 \\
\hline H9A_3 & 6249.75 & 5211.06 & 5593.48 & 27 \\
\hline H9B_3 & 6907.51 & 5444.98 & 5484.77 & 27 \\
\hline H10_3 & 5824.09 & 5409.98 & 3711.02 & 26 \\
\hline H11_3 & 7510.16 & 5168.99 & 4693.35 & 33 \\
\hline H12A_3 & 6222.52 & 4869.09 & 3049.72 & 43 \\
\hline H12B_3 & 7438.34 & 4856.64 & 3643.75 & 43 \\
\hline H14_3 & 6382.46 & 4738.03 & 5250.51 & 26 \\
\hline H15_3 & 6041.29 & 4277.67 & 5389.77 & 27 \\
\hline H17A_3 & 4190.02 & 3948.8 & 4751.16 & 47 \\
\hline H17B_3 & 5111.95 & 3864.69 & 4624.5 & 47 \\
\hline $\mathrm{H} 17 \mathrm{C}^{-} 3$ & 4029.24 & 3884.08 & 3675.95 & 47 \\
\hline
\end{tabular}




$\begin{array}{lllll}\text { H18_3 } & 3287.87 & 4340.84 & 2988.73 & 31 \\ \text { H3_4 } & 2885 & 5772.95 & 9999.33 & 31 \\ \text { H4_4 } & 2226.7 & 6218.5 & 9542.52 & 32 \\ \text { H5_4 } & 2883.92 & 6524.46 & 8888.84 & 31 \\ \text { H6_4 } & 4242.99 & 6403.07 & 8702.6 & 28 \\ \text { H9A_4 } & 6070.08 & 5276.83 & 10128.27 & 29 \\ \text { H9B_4 } & 6680.26 & 5523.74 & 10013.22 & 29 \\ \text { H10_4 } & 5562.7 & 5495.36 & 8238.19 & 25 \\ \text { H11_4 } & 6415.62 & 5116.35 & 7985.71 & 28 \\ \text { H12A_4 } & 7716.91 & 5247.1 & 9950.07 & 33 \\ \text { H12B_4 } & 7976.69 & 5081.64 & 9190.61 & 33 \\ \text { H14_4 } & 6262.71 & 4821.33 & 9755.18 & 25 \\ \text { H15_4 } & 5926.97 & 4373.63 & 10042.83 & 39 \\ \text { H17A_4 } & 3801.02 & 4069.81 & 9367.63 & 39 \\ \text { H17B_4 } & 4918.97 & 3988.3 & 9706.85 & 39 \\ \text { H17C_4 } & 4073.61 & 3951.37 & 8577.83 & 26 \\ \text { H18_4 } & 3096.99 & 4420.9 & 7783.32 & \end{array}$

\section{Experimental}

Single crystals of $\mathrm{C}_{126} \mathrm{H}_{96} \mathrm{~N}_{12} \mathrm{O}_{24} 9 \mathbf{m j}$ were obtained by recrystallization from DCM/hexanes. A suitable crystal was selected and measured on a Bruker D8Venture diffractometer with a PHOTON-II detector. The crystal was kept at $100 \mathrm{~K}$ during data collection. Using Apex3, the data were integrated and corrected for Lorentz and polarization effects using SAINT and corrected for absorption effects using SADABS. ${ }^{21}$ The structure was solved with the ShelXD structure solution program using Dual Space methods. ${ }^{22}$ Least squares minimization refinement was carried out using Olex ${ }^{23}$ with the $\mathrm{XL}^{22}$ refinement package. The structure was refined as a three-component twin. All non-hydrogen atoms were refined with anisotropic atomic displacement parameters. Atomic displacement parameters for the hydrogens were tied to the equivalent isotropic displacement parameter of the atom to which they are bonded $\left(U_{\text {iso }}(\mathrm{H})=1.5 U_{\text {eq }}(\mathrm{C})\right.$ for methyl, $1.2 U_{\text {eq }}(\mathrm{C})$ for all others). The absolute structure was determined using anomalous dispersion. The Flack parameter ${ }^{24}$ was determined from 9960 selected quotients with the XL package. ${ }^{22}$

As determined by single crystal X-ray diffraction, compound 9mj crystallizes in monoclinic space group $B 2_{1}$ (No. 4). The crystals appear hexagonal. Close inspection of the data reveals that the crystal is pseudohexagonal with lattice parameter $a$ nearly equivalent to $c$ and $\beta$ nearly $120^{\circ}$; it is monoclinic with three-fold twinning. This unit cell can be described as Bcentered in order to facilitate the twinned data analysis. The asymmetric unit contains six independent molecules, in all of which $\mathrm{C} 10$ is in the $R$ configuration. The absolute configuration was determined by anomalous dispersion, and the Flack parameter of 0.003(47) indicates that the crystal is enantiopure. ${ }^{24}$

\section{Crystal structure determination of $9 \mathrm{mj}$}

Crystal Data for $\mathrm{C}_{126} \mathrm{H}_{96} \mathrm{~N}_{12} \mathrm{O}_{24}(M=2162.14 \mathrm{~g} / \mathrm{mol})$ : monoclinic, space group B 2yb (No. 4), $a=15.5993(8) \AA, b=$ 49.062(2) $\AA, c=15.6099(8) \AA, \beta=119.896(2)^{\circ}, V=10357.0(9) \AA^{3}, Z=4, T=100 \mathrm{~K}, \mu(\mathrm{CuK} \alpha)=0.802 \mathrm{~mm}^{-1}$, Dcalc $=$ $1.387 \mathrm{~g} / \mathrm{cm}^{3}, 349707$ reflections measured $\left(3.602^{\circ} \leq 2 \Theta \leq 160.338^{\circ}\right), 43677$ unique $\left(R_{\text {int }}=0.0579, R_{\text {sigma }}=0.0357\right)$ which were used in all calculations. The final $R_{1}$ was $0.0440(\mathrm{I}>2 \sigma(\mathrm{I}))$ and $w R_{2}$ was 0.1211 (all data). 


\section{Refinement model description}

Number of restraints -1 , number of constraints - 0 .

Details:

1. Twinned data refinement

Scales: $0.2514 \quad 0.3729 \quad 0.3757$

2. Fixed Uiso

At 1.2 times of: All $\mathrm{C}(\mathrm{H})$ groups, All C(H,H) groups

At 1.5 times of: All $\mathrm{C}(\mathrm{H}, \mathrm{H}, \mathrm{H})$ groups

3.a Ternary $\mathrm{CH}$ refined with riding coordinates:

$\mathrm{C} 10(\mathrm{H} 10), \mathrm{C} 10(\mathrm{H} 10), \mathrm{C} 10(\mathrm{H} 10), \mathrm{C} 10(\mathrm{H} 10), \mathrm{C} 10(\mathrm{H} 10), \mathrm{C} 10(\mathrm{H} 10)$

3.b Secondary $\mathrm{CH} 2$ refined with riding coordinates:

$\mathrm{C} 9(\mathrm{H} 9 \mathrm{~A}, \mathrm{H} 9 \mathrm{~B}), \mathrm{C} 9(\mathrm{H} 9 \mathrm{~A}, \mathrm{H9B}), \mathrm{C} 9(\mathrm{H} 9 \mathrm{~A}, \mathrm{H} 9 \mathrm{~B}), \mathrm{C} 9(\mathrm{H} 9 \mathrm{~A}, \mathrm{H9B}), \mathrm{C} 9(\mathrm{H} 9 \mathrm{~A}, \mathrm{H} 9 \mathrm{~B}), \mathrm{C} 9(\mathrm{H} 9 \mathrm{~A}, \mathrm{H} 9 \mathrm{~B})$

3.C Aromatic/amide $\mathrm{H}$ refined with riding coordinates:

$\mathrm{C} 3(\mathrm{H} 3), \mathrm{C} 4(\mathrm{H} 4), \mathrm{C} 5(\mathrm{H} 5), \mathrm{C} 6(\mathrm{H} 6), \mathrm{C} 11(\mathrm{H} 11), \mathrm{C} 14(\mathrm{H} 14), \mathrm{C} 15(\mathrm{H} 15), \mathrm{C} 18(\mathrm{H} 18), \mathrm{C} 3(\mathrm{H} 3)$,

$\mathrm{C} 4(\mathrm{H} 4), \mathrm{C} 5(\mathrm{H} 5), \mathrm{C} 6(\mathrm{H} 6), \mathrm{C} 11(\mathrm{H} 11), \mathrm{C} 14(\mathrm{H} 14), \mathrm{C} 15(\mathrm{H} 15), \mathrm{C} 18(\mathrm{H} 18), \mathrm{C} 3(\mathrm{H} 3), \mathrm{C} 4(\mathrm{H} 4)$,

$\mathrm{C} 5(\mathrm{H} 5), \mathrm{C} 6(\mathrm{H} 6), \mathrm{C} 11(\mathrm{H} 11), \mathrm{C} 14(\mathrm{H} 14), \mathrm{C} 15(\mathrm{H} 15), \mathrm{C} 18(\mathrm{H} 18), \mathrm{C} 3(\mathrm{H} 3), \mathrm{C} 4(\mathrm{H} 4), \mathrm{C} 5(\mathrm{H} 5)$,

$\mathrm{C} 6(\mathrm{H} 6), \mathrm{C} 11(\mathrm{H} 11), \mathrm{C} 14(\mathrm{H} 14), \mathrm{C} 15(\mathrm{H} 15), \mathrm{C} 18(\mathrm{H} 18), \mathrm{C} 3(\mathrm{H} 3), \mathrm{C} 4(\mathrm{H} 4), \mathrm{C} 5(\mathrm{H} 5), \mathrm{C} 6(\mathrm{H} 6)$,

$\mathrm{C} 11$ (H11) , C14(H14), C15(H15), C18(H18), C3(H3), C4(H4), C5(H5), C6(H6),

$\mathrm{C} 11$ (H11), C14(H14), C15(H15), C18(H18)

3. $\mathrm{d} \mathrm{X}=\mathrm{CH} 2$ refined with riding coordinates:

C12 (H12A, H12B), C12 (H12A,H12B), C12 (H12A,H12B), C12 (H12A,H12B), C12 (H12A,

H12B), C12 (H12A, H12B)

3.e Idealised Me refined as rotating group:

C17 (H17A, H17B,H17C) , C17 (H17A, H17B,H17C), C17 (H17A, H17B,H17C),

C17 (H17A, H17B, H17C), C17 (H17A, H17B, H17C), C17 (H17A, H17B, H17C) 


\section{References}

(1) Varano, F.; Catarzi, D.; Colotta, V.; Calabri, F. R.; Lenzi, O.; Filacchioni, G.; Galli, A.; Costagli, C.; Deflorian, F.; Moro, S. 1-Substituted pyrazolo[1,5-c]quinazolines as novel Gly/NMDA receptor antagonists: Synthesis, biological evaluation, and molecular modeling study. Bioorg. Med. Chem. 2005, 13, 5536-5549.

(2) Ziegler, E.; Wolf, R.; Kappe, T. Synthesen von Heterocyclen, 66. Mitt.: Eine einfache Synthese des 4Hydroxycarbostyrils und seiner Derivate. Monatsh. Chem. 1965, 96, 418-422.

(3) Herscheid, J. D. M.; Colstee, J. H.; Ottenheijm, H. C. J. 1,4-Dihydroxy-2,5-dioxopiperazines from activated Nhydroxyamino acids. J. Org. Chem. 1981, 46, 3346-3348.

(4) Klein, L. L.; Tufano, M. D. Synthesis of substituted isatins. Tetrahedron Lett. 2013, 54, 1008-1011.

(5) Mann, F. G.; Turnbull, J. H. 162. Xanthones and thioxanthones. Part I. The synthesis of 2- and 3dialkylaminoalkylamino-derivatives. J. Chem. Soc. 1951, 747-756.

(6) Comess, K. M.; Erickson, S. A.; Henkin, J.; Kalvin, D. M.; Kawai, M.; Kim, K. H.; BaMaung, N. Y.; Park, C. H.; Sheppard, G. S.; Vasudevan, A.; Wang, J.; Barnes, D. M.; Fidanze, S. D.; Kolaczkowski, L.; Mantei, R. A.; Park, D. C.; Sanders, W. J.; Tedrow, J. S.; Wang, G. T. Sulfonamides Having Antiangiogenic and Anticancer Activity. U.S. Pat. Appl. US 2004/0157836 A1, Aug 12, 2004.

(7) Chupakhin, O. N.; Rusinov, V. L.; Beresnev, D. G.; Neunhoeffer, H. Reactions of 1,2,4-triazin-5(2H)-ones with phenols and aromatic amines. J. Heterocyclic Chem. 1997, 34, 573-578.

(8) Marion, N.; Gealageas, R.; Nolan, S. P. [(NHC)Au ${ }^{\mathrm{I}}$-Catalyzed Rearrangement of Allylic Acetates. Org. Lett. 2007, 9, 2653-2656.

(9) Fuchter, M. J.; Levy, J.-N. One-Pot Formation of Allylic Chlorides from Carbonyl Derivatives. Org. Lett. 2008, 10, 4919-4922.

(10) Schmidt, B. Ruthenium-Catalyzed Olefin Metathesis Double-Bond Isomerization Sequence. J. Org. Chem. 2004, 69, $7672-7687$.

(11) Hu, Q.; Negri, M.; Jahn-Hoffmann, K.; Zhuang, Y.; Olgen, S.; Bartels, M.; Müller-Vieira, U.; Lauterbach, T.; Hartmann, R. W. Synthesis, biological evaluation, and molecular modeling studies of methylene imidazole substituted biaryls as inhibitors of human 17 $\alpha$-hydroxylase-17,20-lyase (CYP17) - Part II: Core rigidification and influence of substituents at the methylene bridge. Bioorg. Med. Chem. 2008, 16, 7715-7727.

(12) Musolino, S. F.; Ojo, O. S.; Westwood, N. J.; Taylor, J. E.; Smith, A. D. Isothiourea-Catalysed Acylative Kinetic

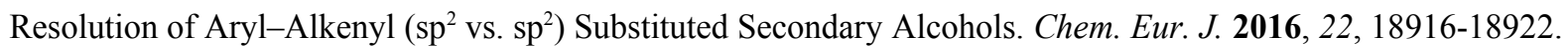

(13) Tu, Z.; Li, S.; Cui, J.; Xu, J.; Taylor, M.; Ho, D.; Luedtke, R. R.; Mach, R. H. Synthesis and Pharmacological Evaluation of Fluorine-Containing D3 Dopamine Receptor Ligands. J. Med. Chem. 2011, 54, 1555-1564.

(14) Capuano, L.; Drescher, S.; Hammerer, V.; Hanisch, M. Neue Heterocyclensynthesen durch Wittig-Reaktion, II. 2,2'Biindolyle; 1,2-Di(2-indolyl)- und 1,2-Di(1-benzofuran-2-yl)ethylene. Chem. Ber. 1988, 121, 2259-2261.

(15) Schweizer, E. E.; Creasy, W. S.; Light, K. K.; Shaffer, E. T. Reactions of phosphorous compounds. XX. Reactions of furfuryl-, dihydrofurfuryl, and tetrahydrofurfuryltriphenylphosphonium bromide. J. Org. Chem. 1969, 34, 212-218.

(16) Sun, L.; Tran, N.; Tang, F.; App, H.; Hirth, P.; McMahon, G.; Tang, C. Synthesis and Biological Evaluations of 3Substituted Indolin-2-ones: A Novel Class of Tyrosine Kinase Inhibitors That Exhibit Selectivity toward Particular Receptor Tyrosine Kinases. J. Med. Chem. 1998, 41, 2588-2603.

(17) Konkel, M. J.; Lagu, B.; Boteju, L. W.; Jimenez, H.; Noble, S.; Walker, M. W.; Chandrasena, G.; Blackburn, T. P.; Nikam, S. S.; Wright, J. L.; Kornberg, B. E.; Gregory, T.; Pugsley, T. A.; Akunne, H.; Zoski, K.; Wise, L. D. 3Arylimino-2-indolones Are Potent and Selective Galanin $\mathrm{GAL}_{3}$ Receptor Antagonists. J. Med. Chem. 2006, 49, 3757-3758.

(18) Dai, Z.; Li, S.; Li, Y.; Feng, L.; Ma, C. Metal-free synthesis of benzimidazo[1,2-c]quinazolin-6-ones with indole and benzenediamine oxidized by I2/TBHP. Tetrahedron 2019, 75, 2012-2017. 
(19) Tangella, Y.; Manasa, K. L.; Krishna, N. H.; Sridhar, B.; Kamal, A.; Babu, B. N. Regioselective Ring Expansion of Isatins with In Situ Generated a-Aryldiazomethanes: Direct Access to Viridicatin Alkaloids. Org. Lett. 2018, 20, 3639-3642.

(20) Manley, P. J.; Bilodeau, M. T. A New Synthesis of Naphthyridinones and Quinolinones: Palladium-Catalyzed Amidation of o-Carbonyl-Substituted Aryl Halides. Org. Lett. 2004, 6, 2433-2435.

(21) Bruker-AXS (2016). APEX3. Version 2016.9-0. Madison, Wisconsin.

(22) Sheldrick, G. M. A Short History of SHELX. Acta Cryst. 2008, A64, 112-122.

(23) Dolomanov, O. V.; Bourhis, L. J.; Gildea, R. J.; Howard, J. A. K.; Puschmann, H. OLEX2: A Complete Structure Solution, Refinement, and Analysis Program. J. Appl. Cryst. 2009, 42, 339-341.

(24) Parsons, S.; Flack, H. D.; Wagner, T. Use of Intensity Quotients and Differences in Absolute Structure Refinement. Acta Cryst. 2013, B69, 249-259. 


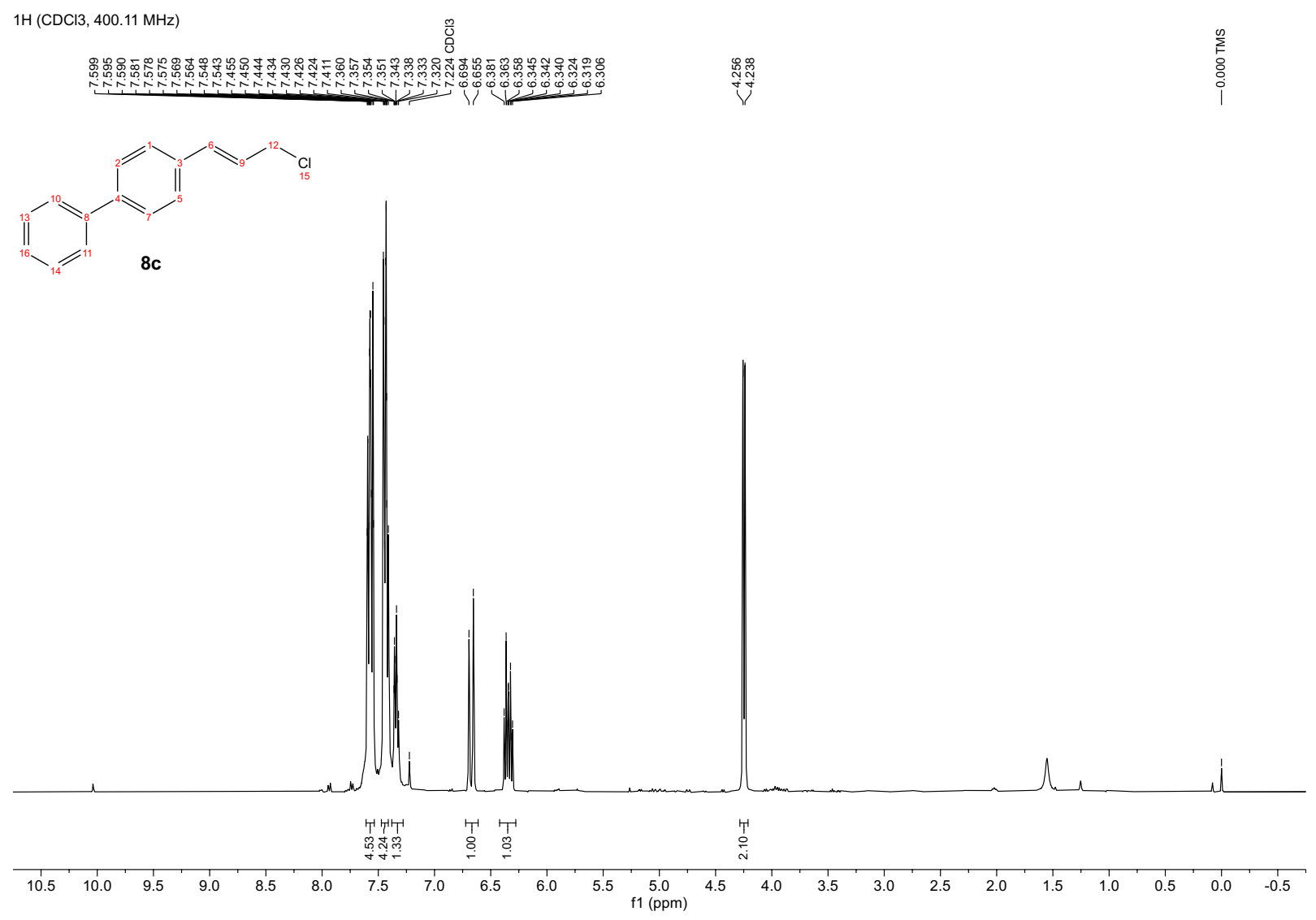

$13 \mathrm{C}(\mathrm{CDCl} 3,100.62 \mathrm{MHz})$
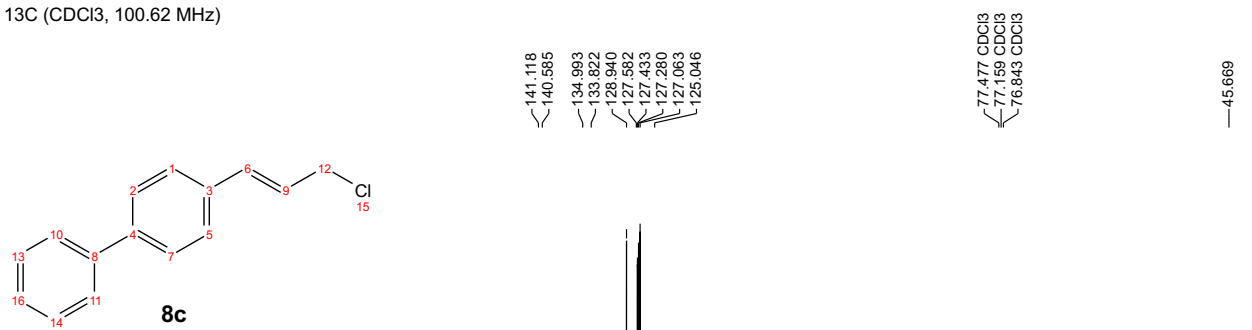

il

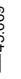

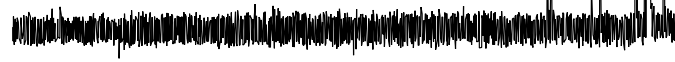

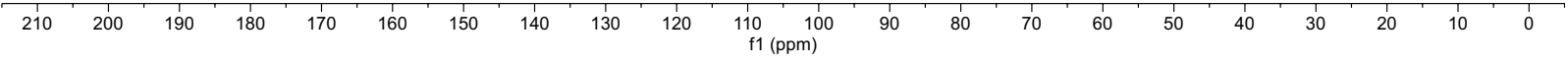



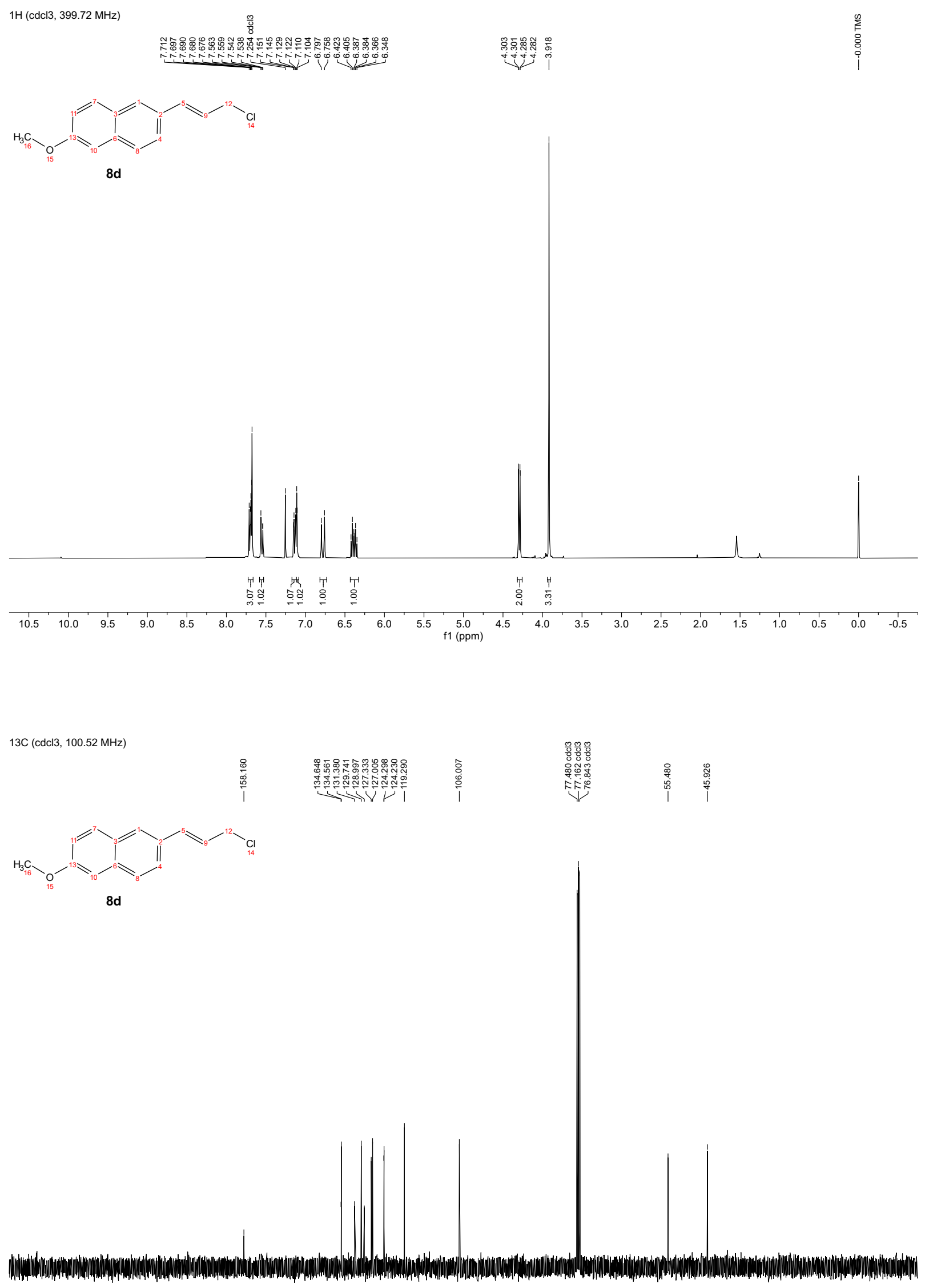

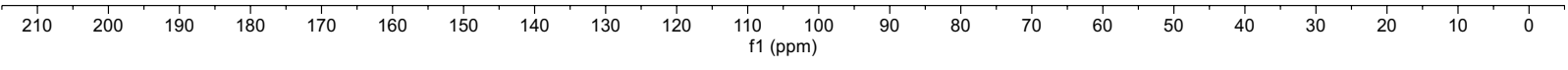


<smiles>[2H]C1([Hg])OC(=O)C(CC=CCP)(CC=Cc2ccccc2)C(=O)O1</smiles>

on

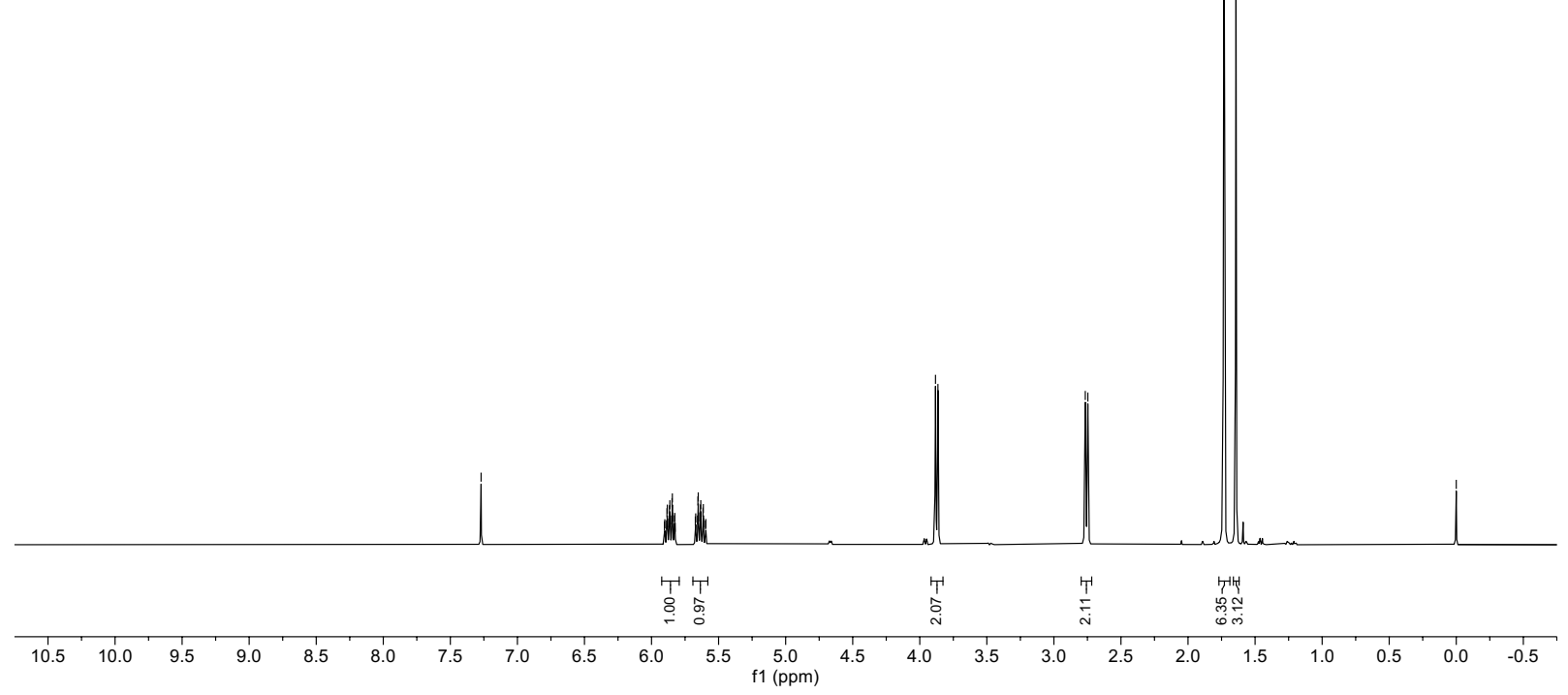

$13 \mathrm{C}(\mathrm{cdcl} 3,100.52 \mathrm{MHz})$

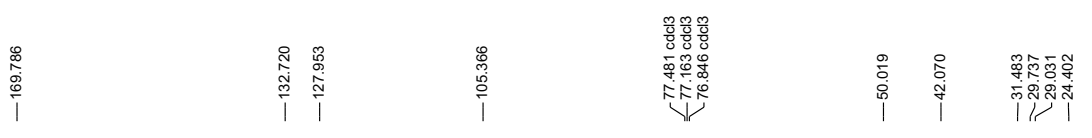

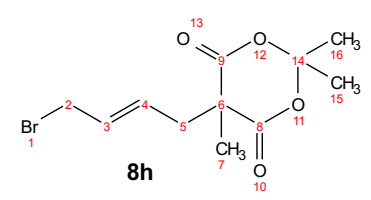

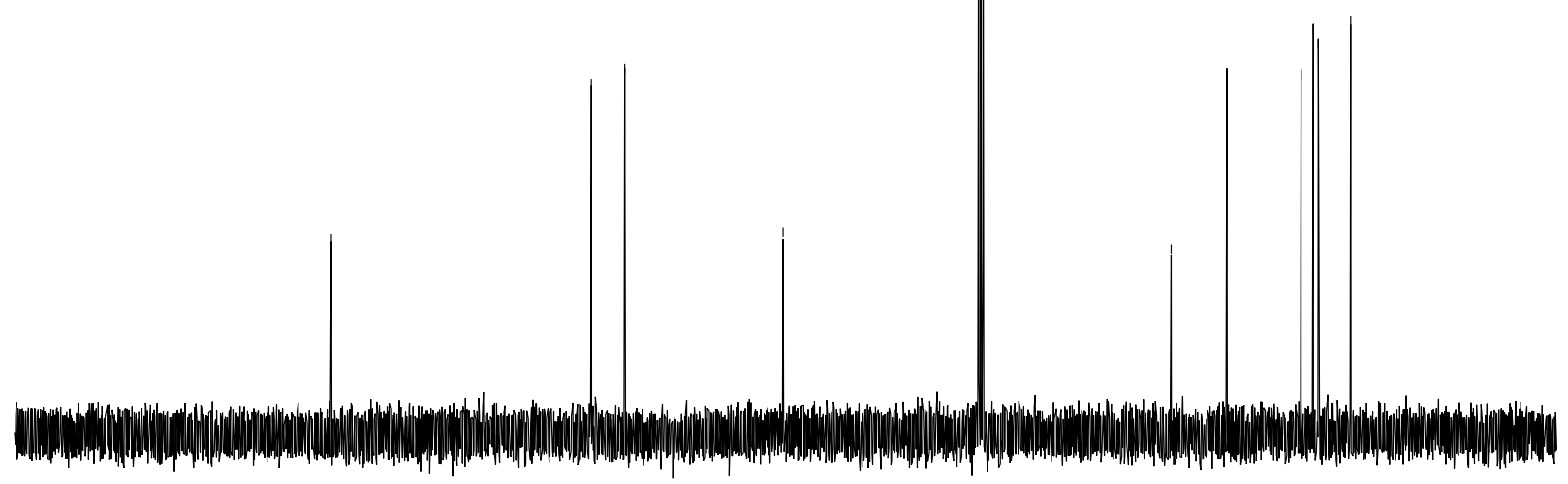

\begin{tabular}{lllllllllllllllllllll}
\hline 210 & 200 & 190 & 180 & 170 & 160 & 150 & 140 & 130 & 120 & $\begin{array}{c}110 \\
\mathrm{f} 1(\mathrm{ppm})\end{array}$ & 90 & 80 & 70 & 60 & 50 & 40 & 30 & 20 & 10 & 0
\end{tabular} 


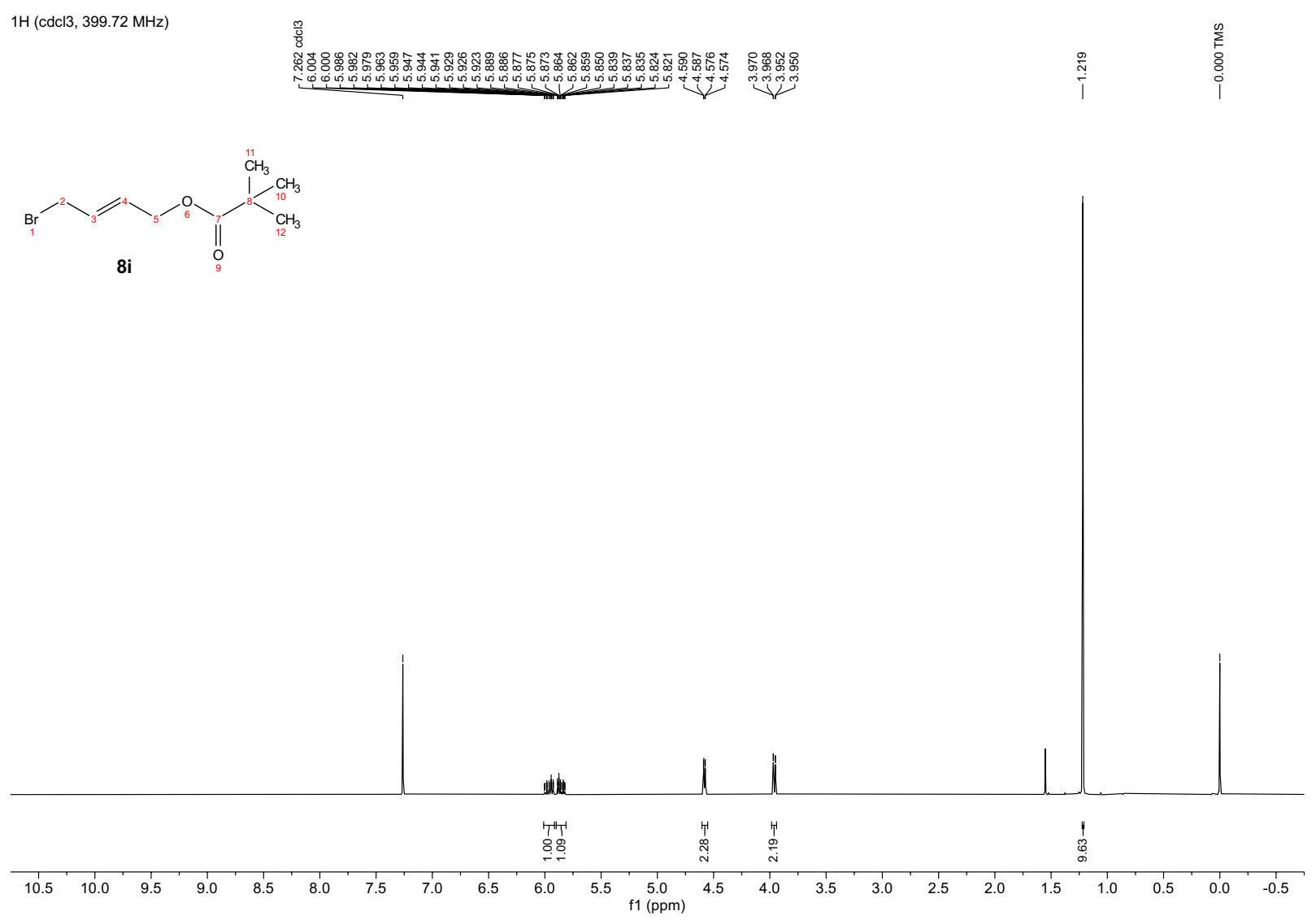

$13 \mathrm{C}(\mathrm{cdcl} 3,100.52 \mathrm{MHz})$
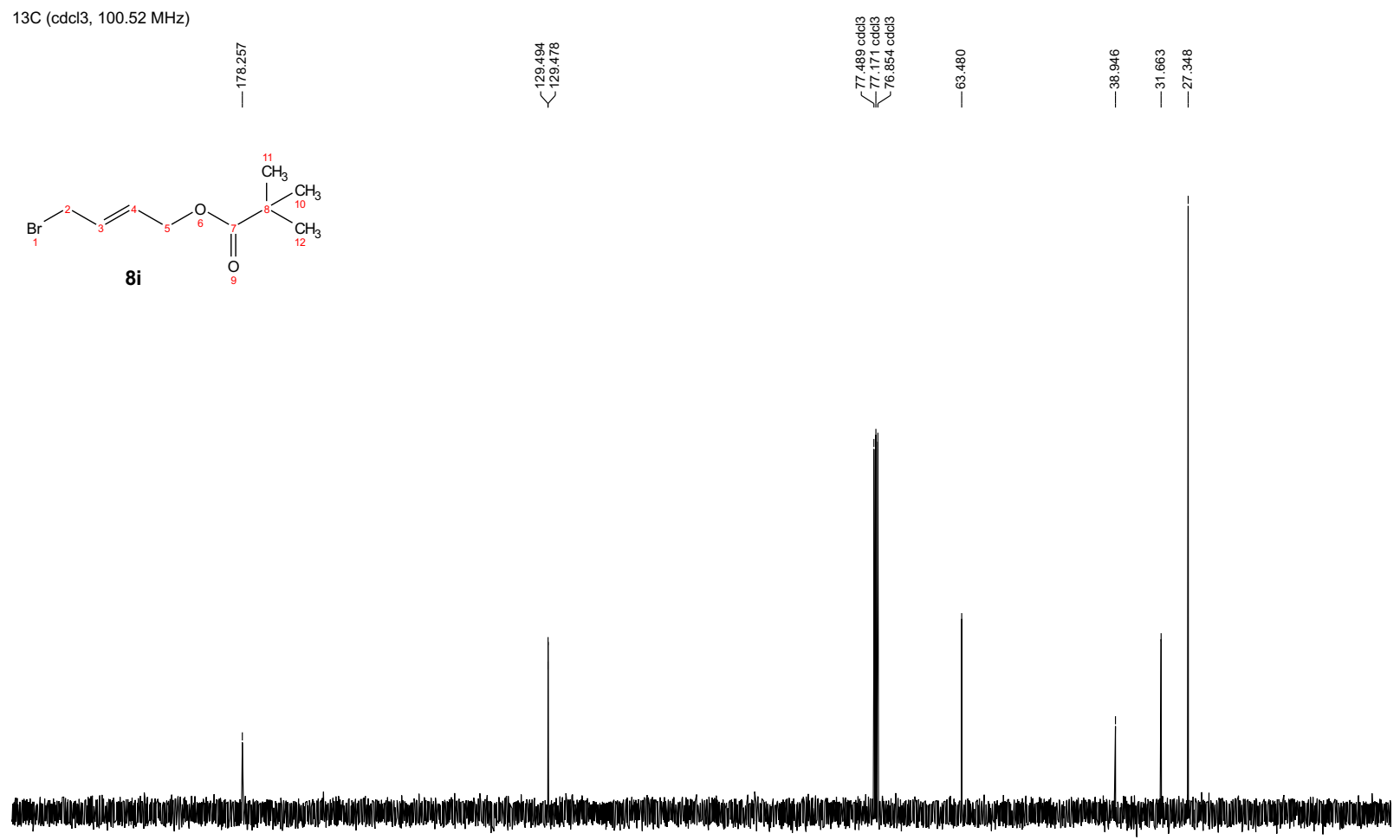

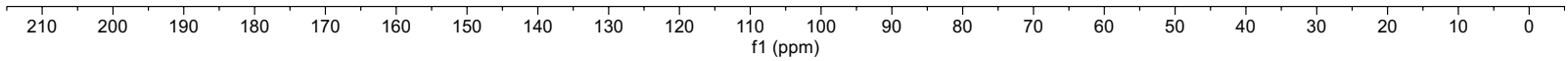


$1 \mathrm{H}(\mathrm{CDCl} 3,299.95 \mathrm{MHz})$

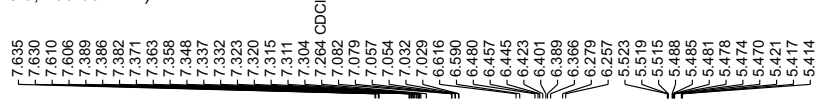
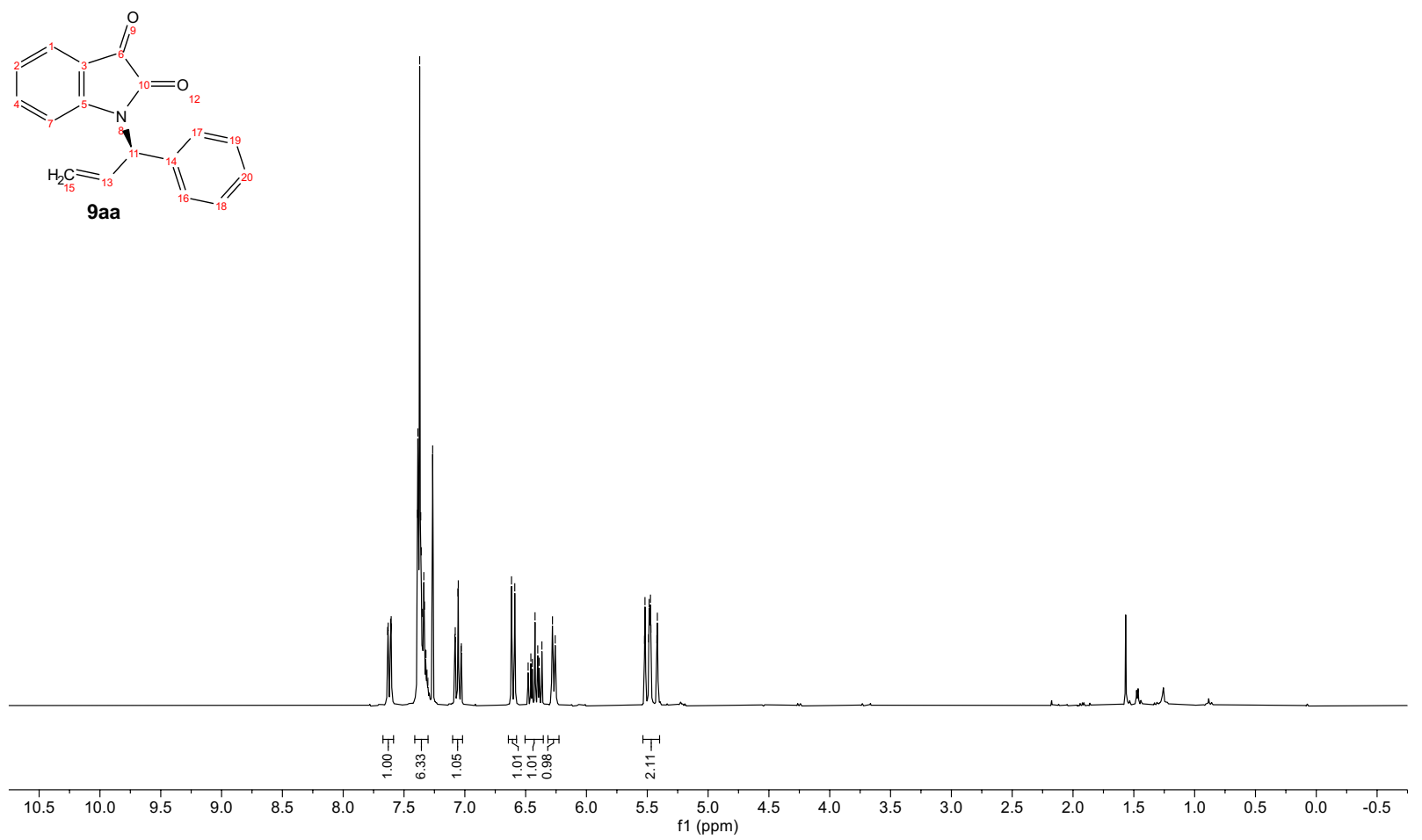

$13 \mathrm{C}(\mathrm{CDCl} 3,100.62 \mathrm{MHz})$

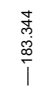

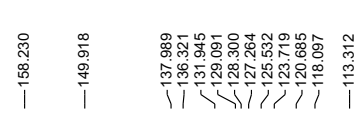

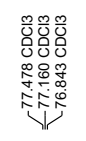

i̊
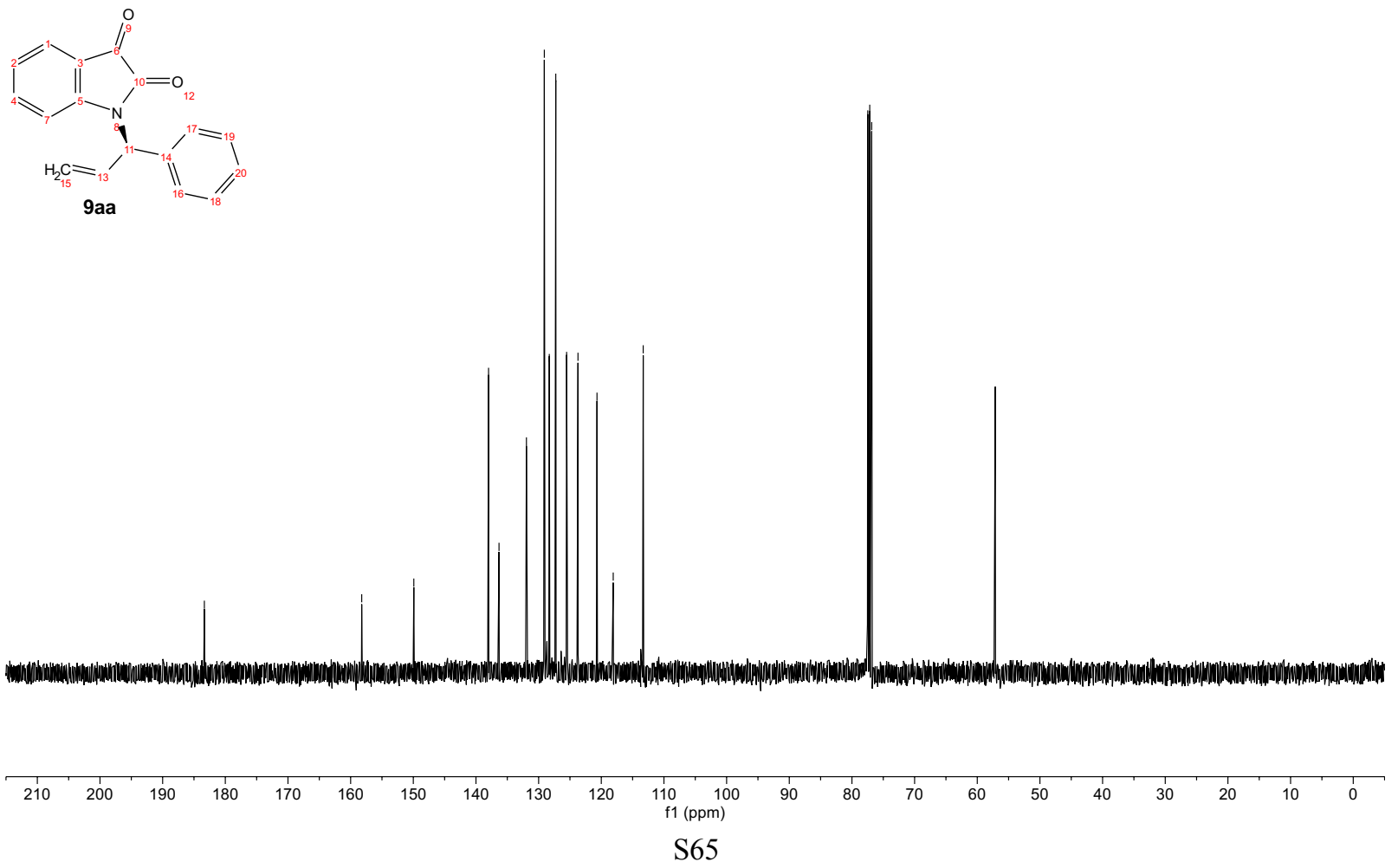
$1 \mathrm{H}(\mathrm{CDCl} 3,299.95 \mathrm{MHz})$

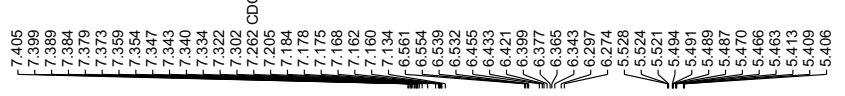

0
$\vdots$
$\vdots$
0
0
0
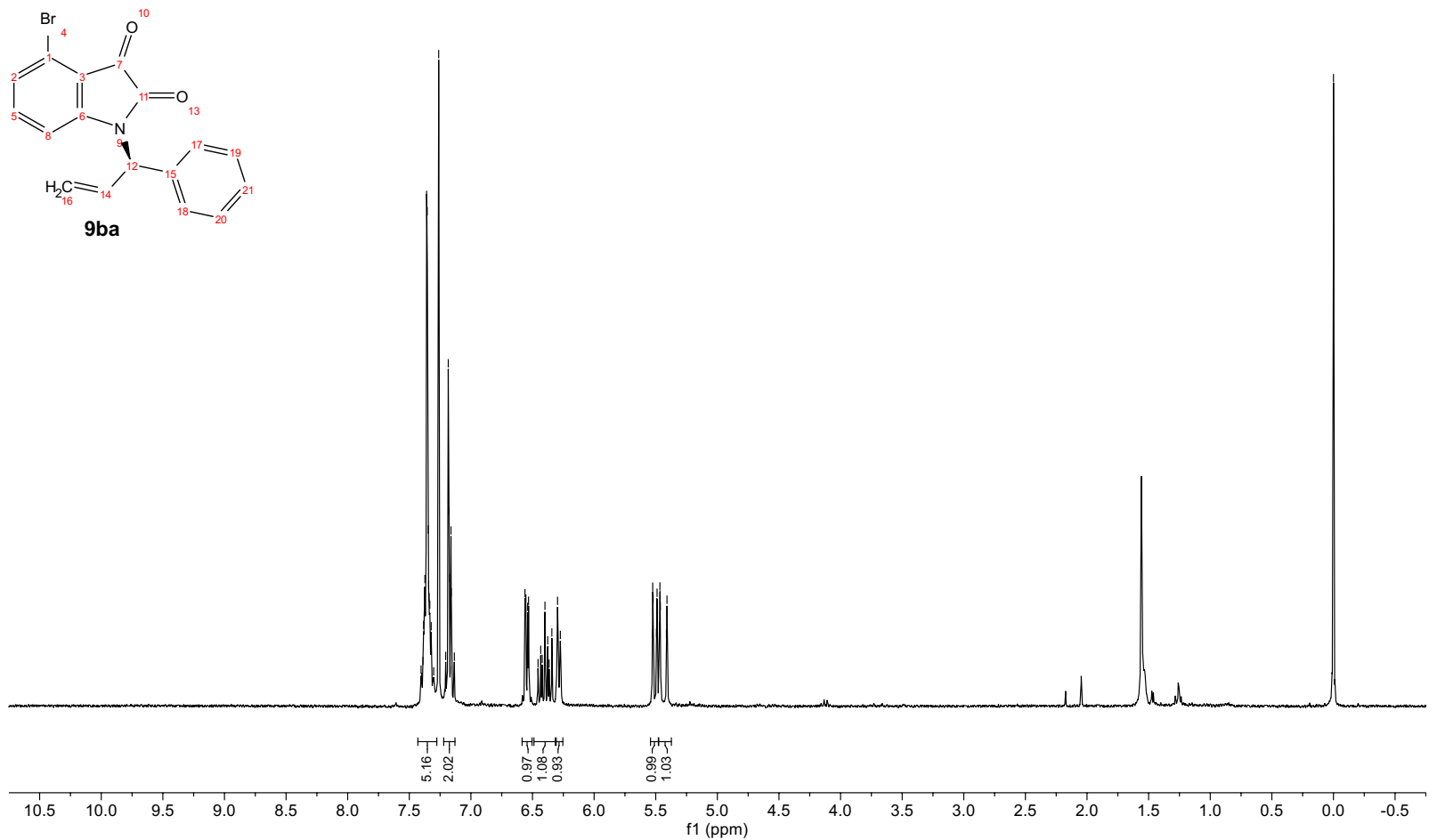

$13 \mathrm{C}(\mathrm{CDCl} 3,100.62 \mathrm{MHz})$

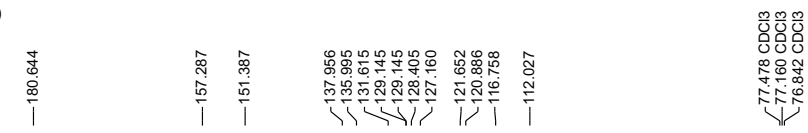

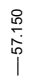
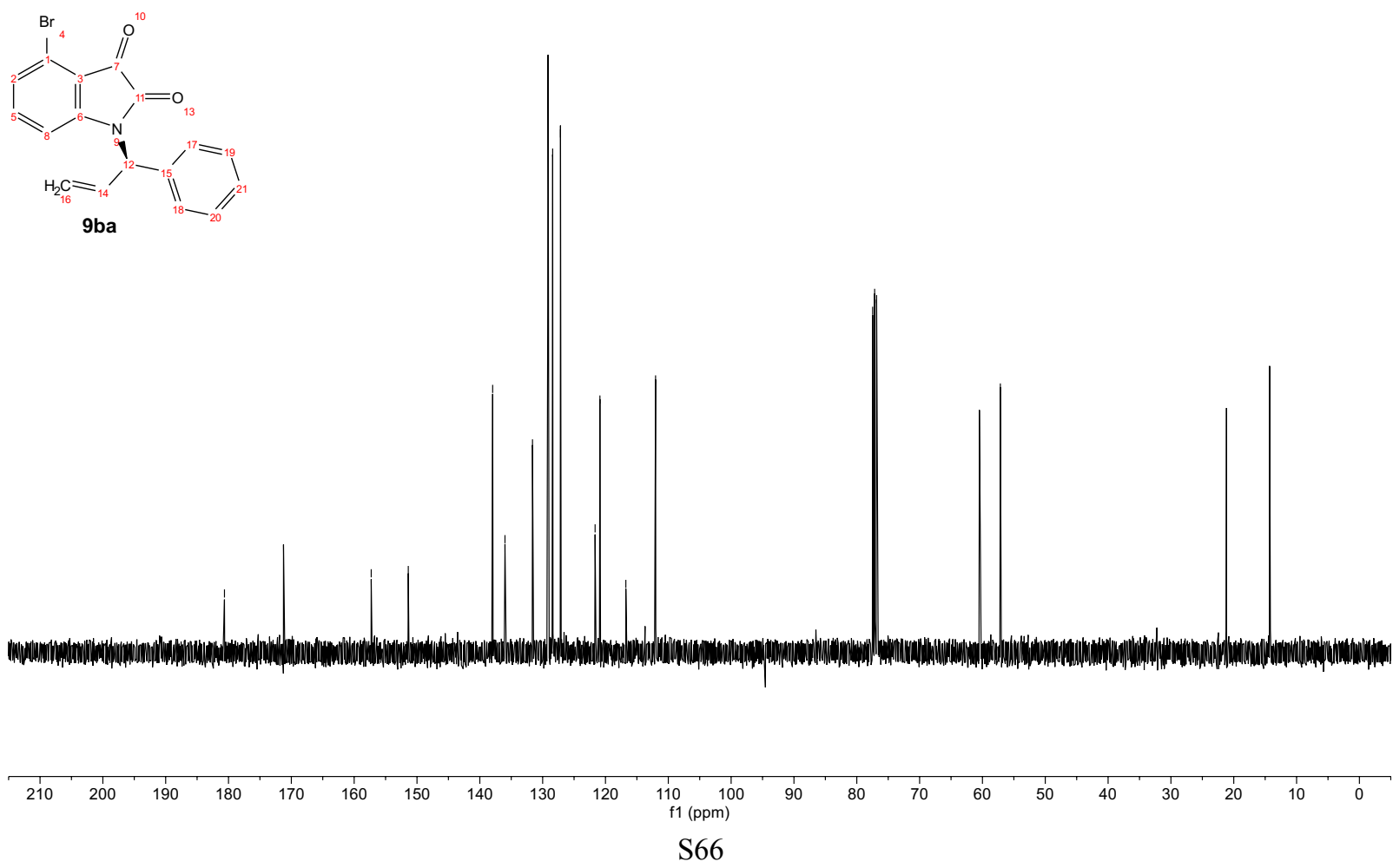


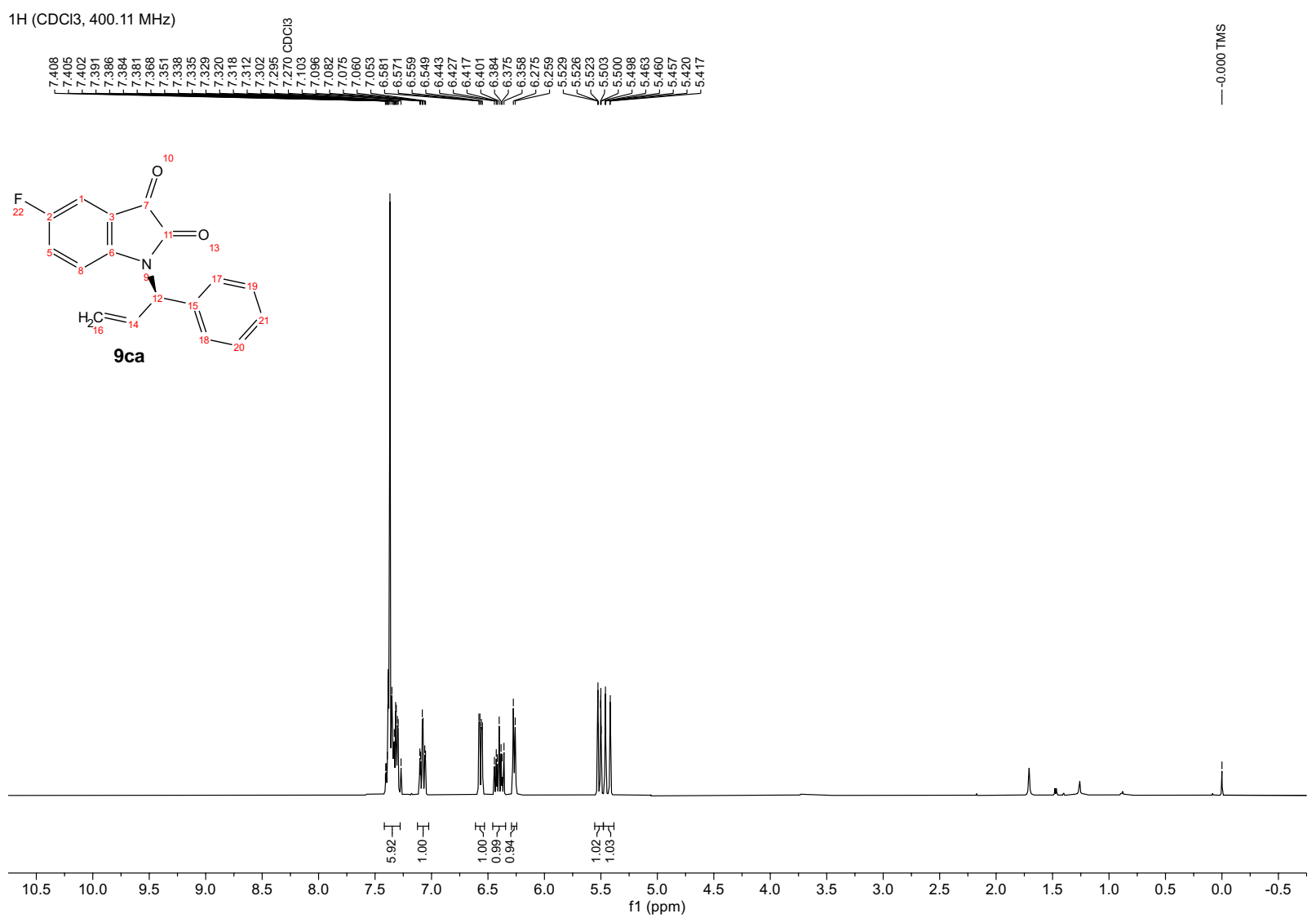

$13 \mathrm{C}(\mathrm{CDCl} 3,100.62 \mathrm{MHz})$
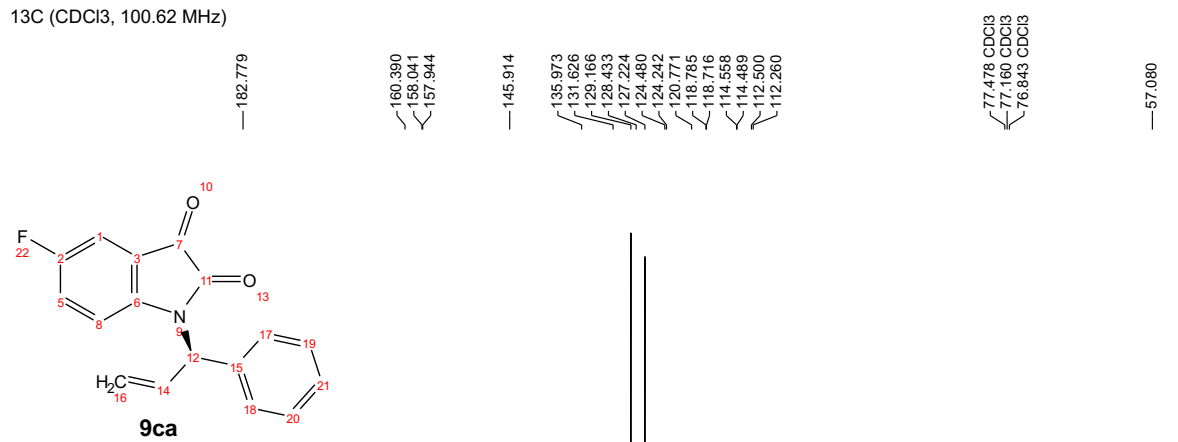

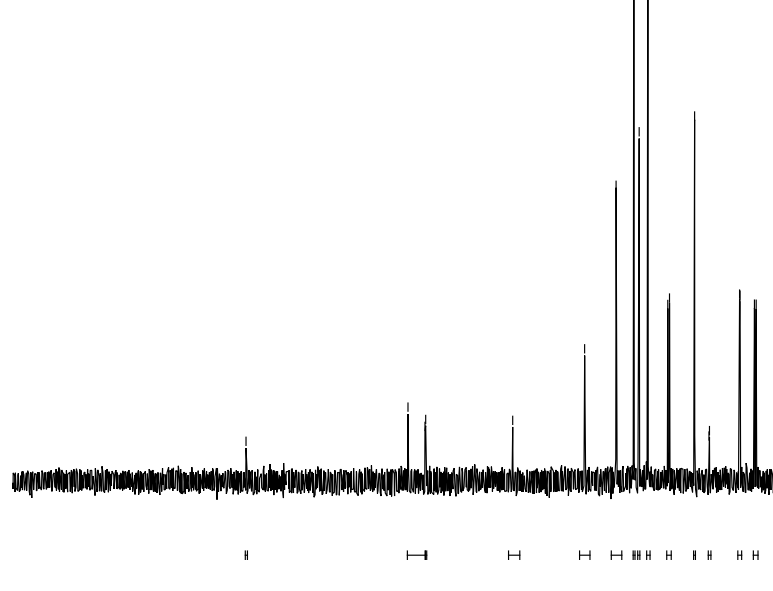

\begin{tabular}{lllllllllllllllllllll}
\hline 210 & 200 & 190 & 180 & 170 & 160 & 150 & 140 & 130 & 120 & $\begin{array}{c}110 \\
\mathrm{f} 1(\mathrm{ppm})\end{array}$ & 90 & 80 & 70 & 60 & 50 & 40 & 30 & 20 & 10 & 0
\end{tabular}




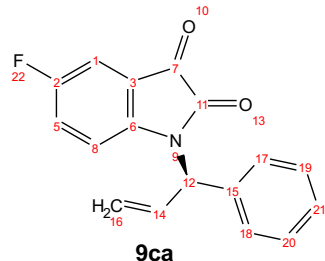

$9 c a$

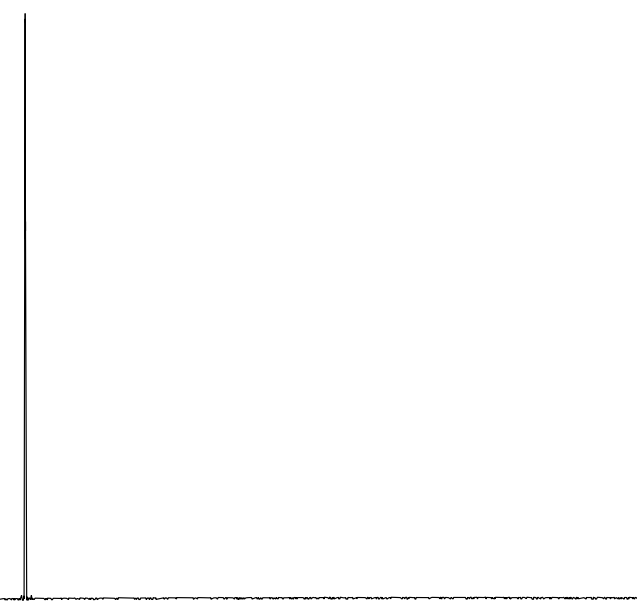

$\longmapsto$

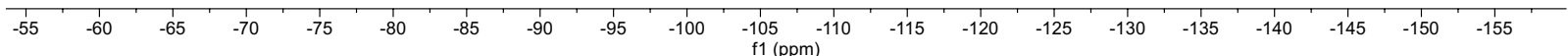




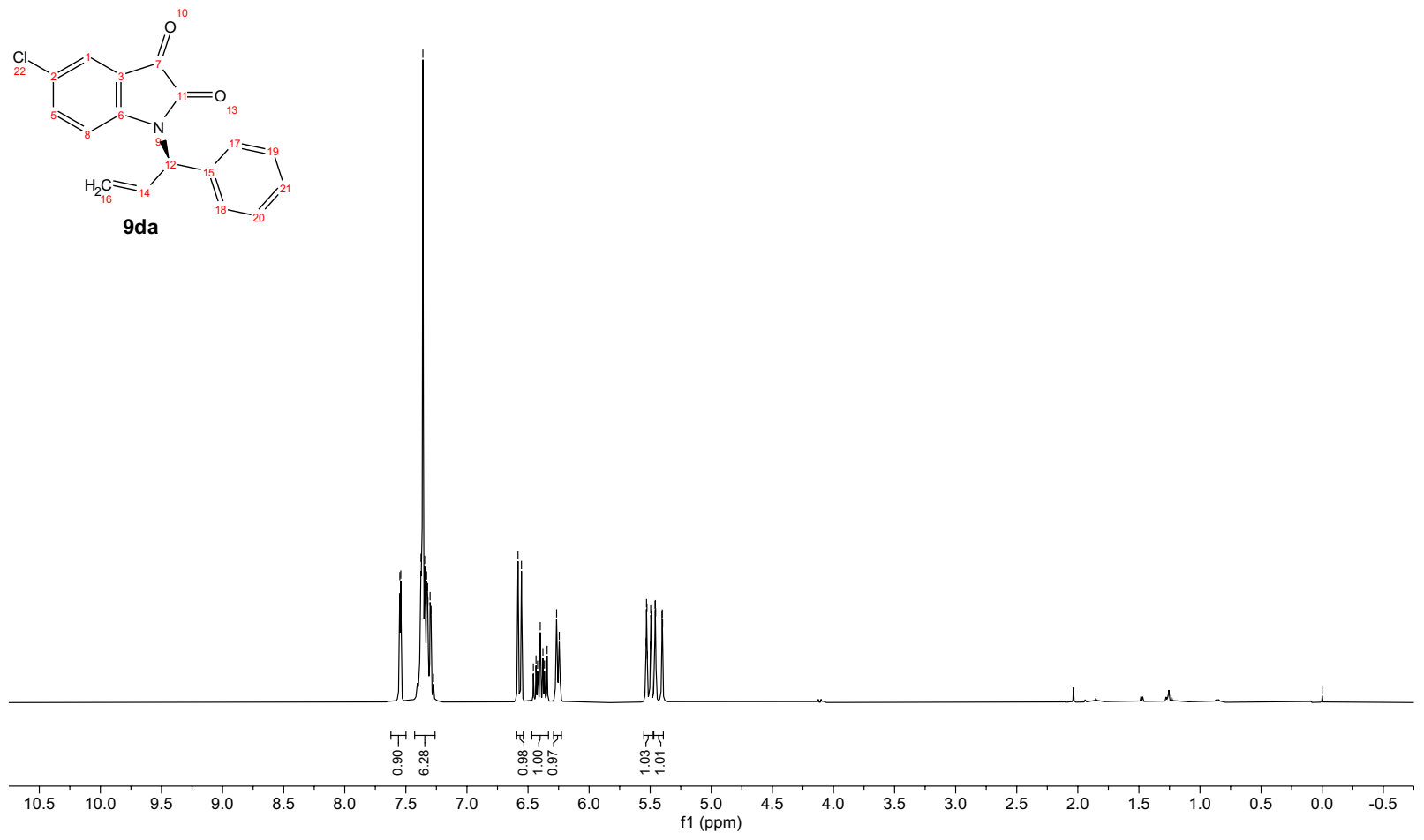

$13 \mathrm{C}(\mathrm{CDCl} 3,100.62 \mathrm{MHz})$

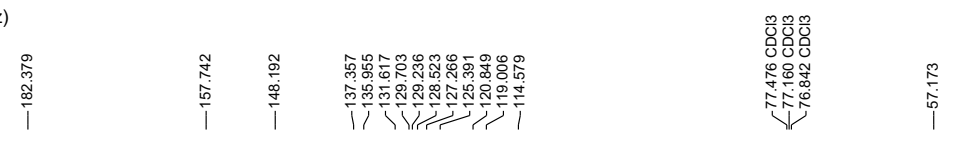

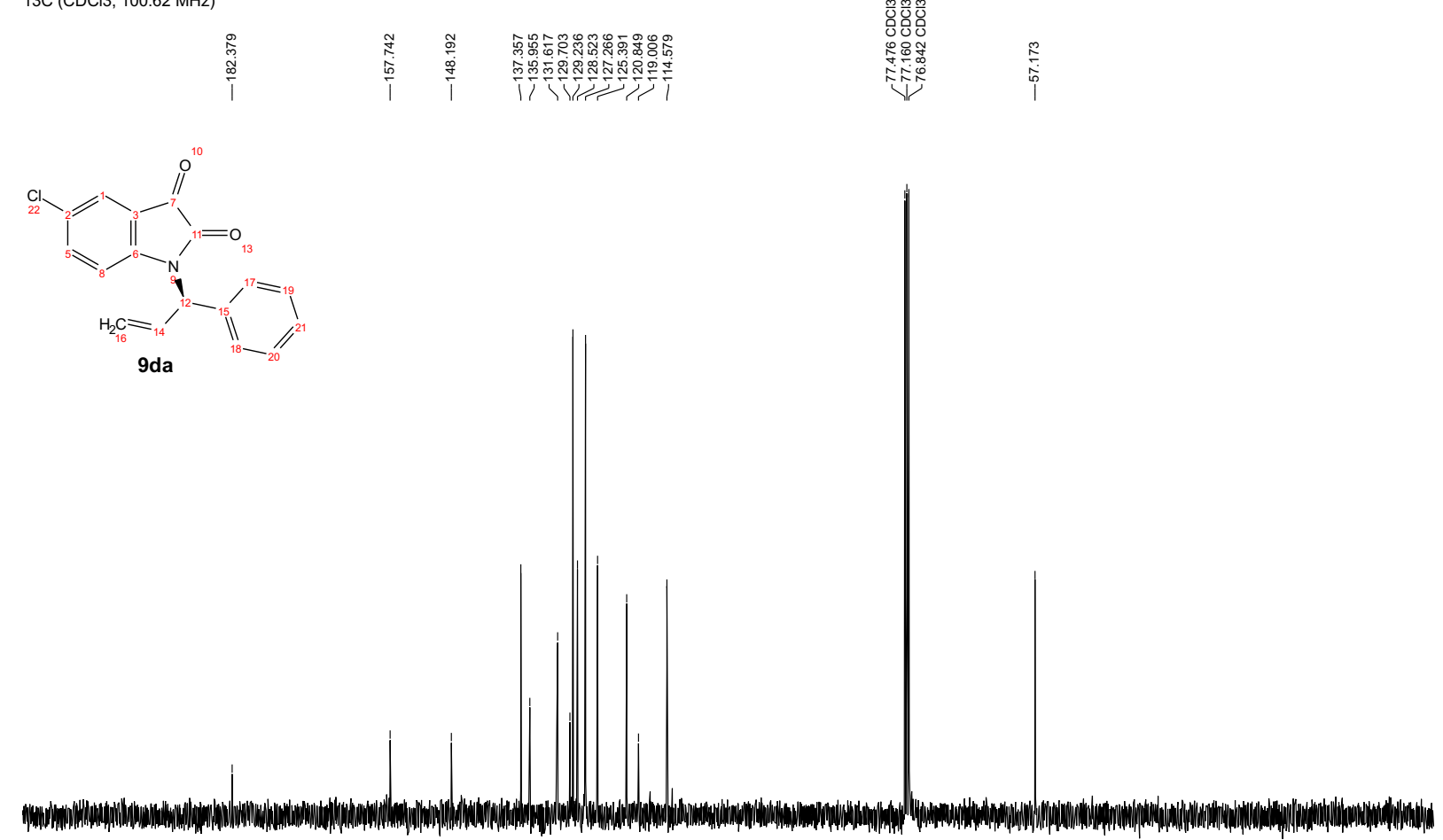

9da

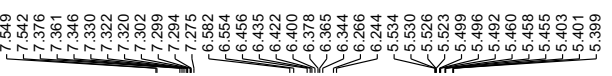

\begin{tabular}{llllllllllllllllllllllll}
\hline 10 & 200 & 190 & 180 & 170 & 160 & 150 & 140 & 130 & 120 & $\begin{array}{c}110 \\
\mathrm{f} 1(\mathrm{ppm})\end{array}$ & 90 & 80 & 70 & 60 & 50 & 40 & 30 & 20 & 10 & 0
\end{tabular} 


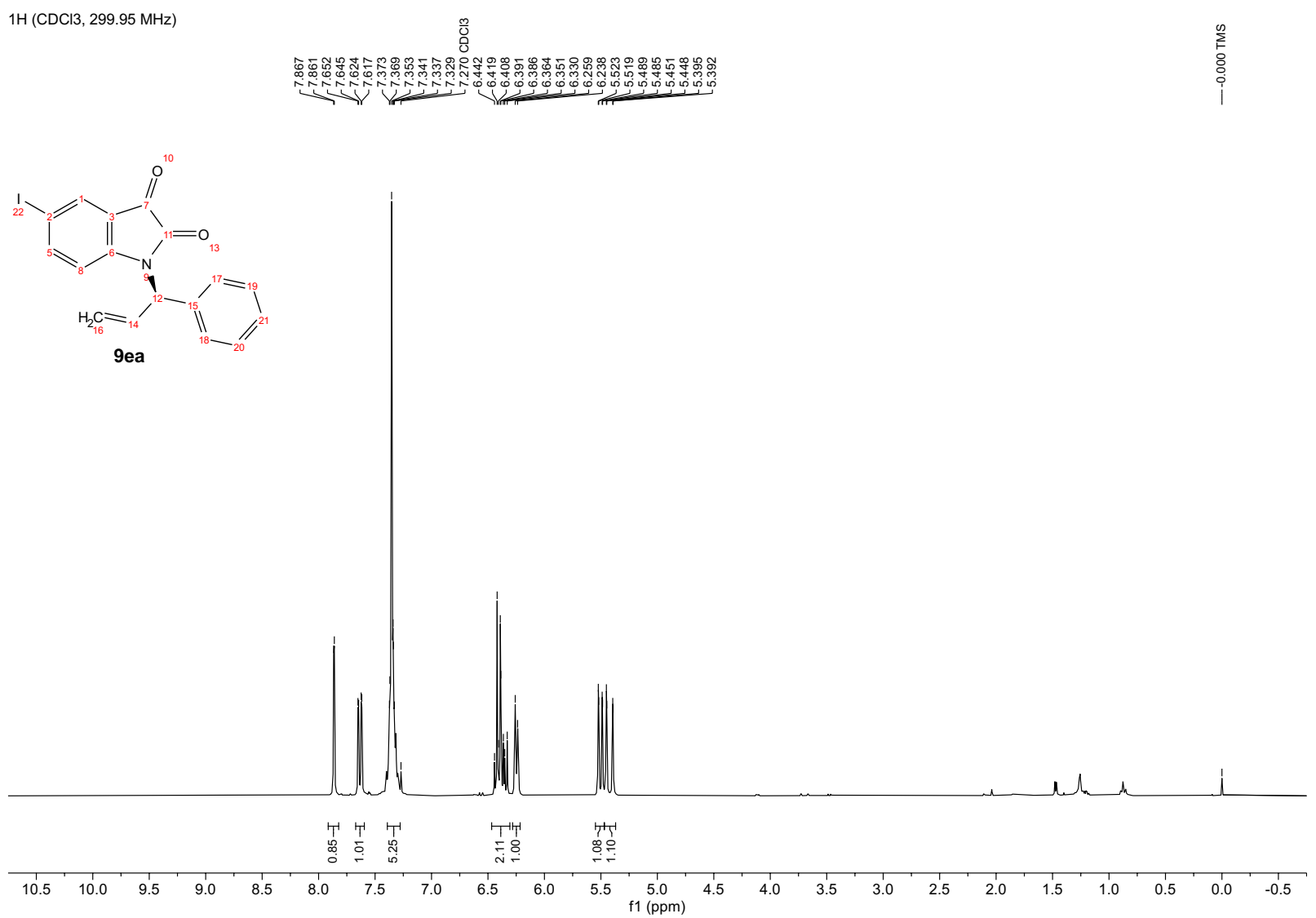

$13 \mathrm{C}(\mathrm{CDCl} 3,75.43 \mathrm{MHz})$

\begin{tabular}{|c|c|c|c|c|c|c|}
\hline i & $\frac{\substack{0\\
}}{i}$ & 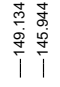 & 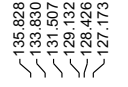 & 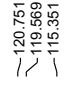 & $\begin{array}{l}\stackrel{0}{0} \\
\stackrel{\infty}{\infty} \\
0 \\
\mid\end{array}$ & 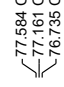 \\
\hline
\end{tabular}
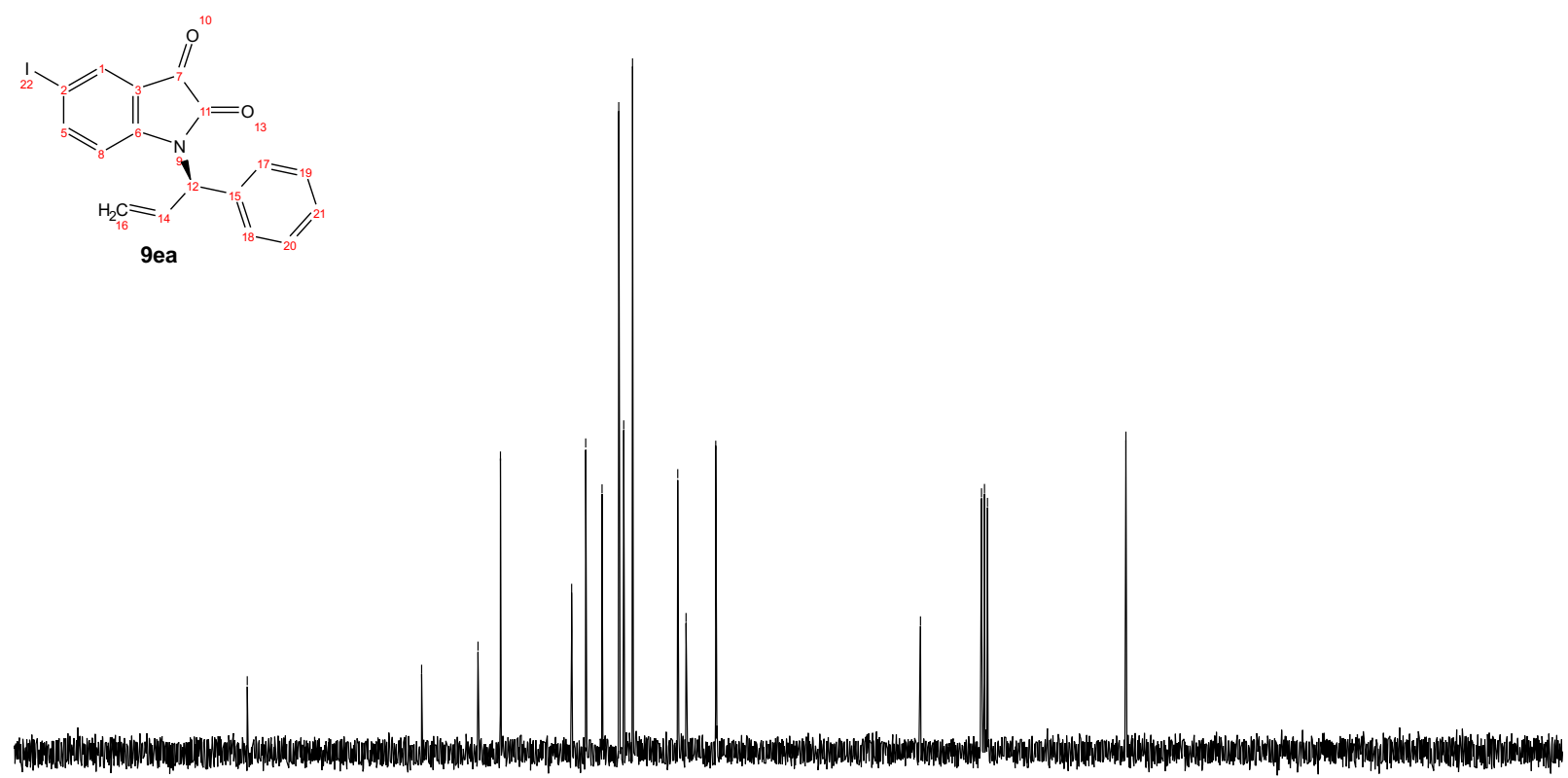

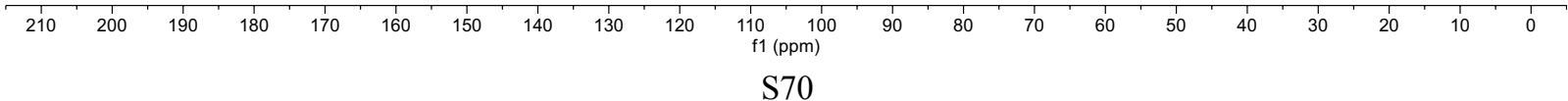




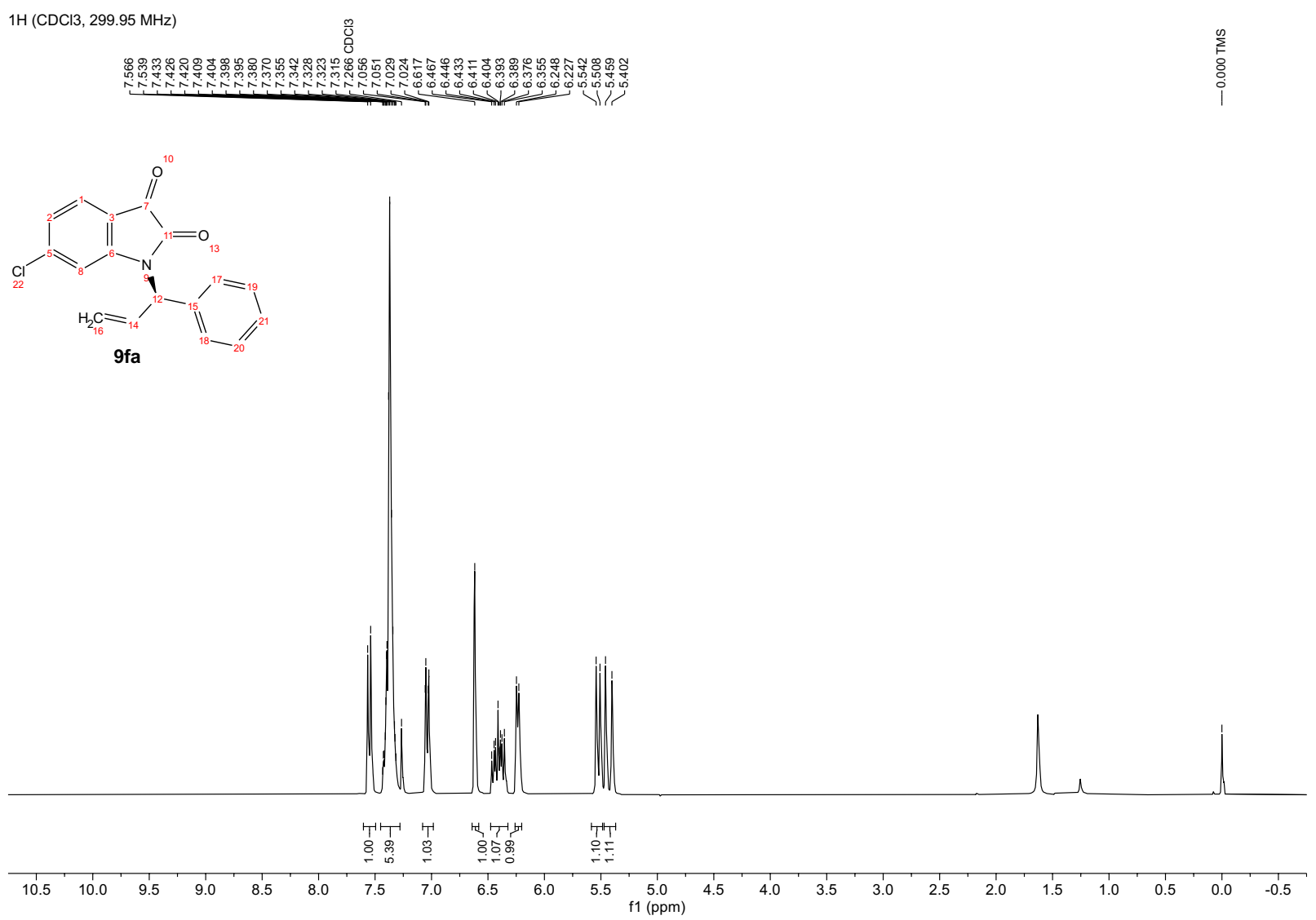

$13 \mathrm{C}(\mathrm{cdcl} 3,100.52 \mathrm{MHz})$

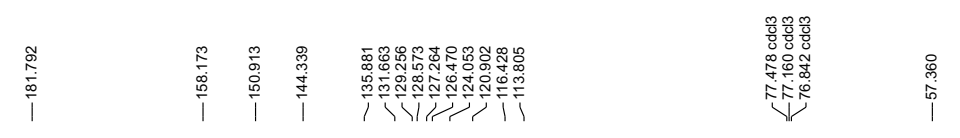<smiles>C=NN1C(=O)S(=O)N2C=CC(Cl)=CN12</smiles>

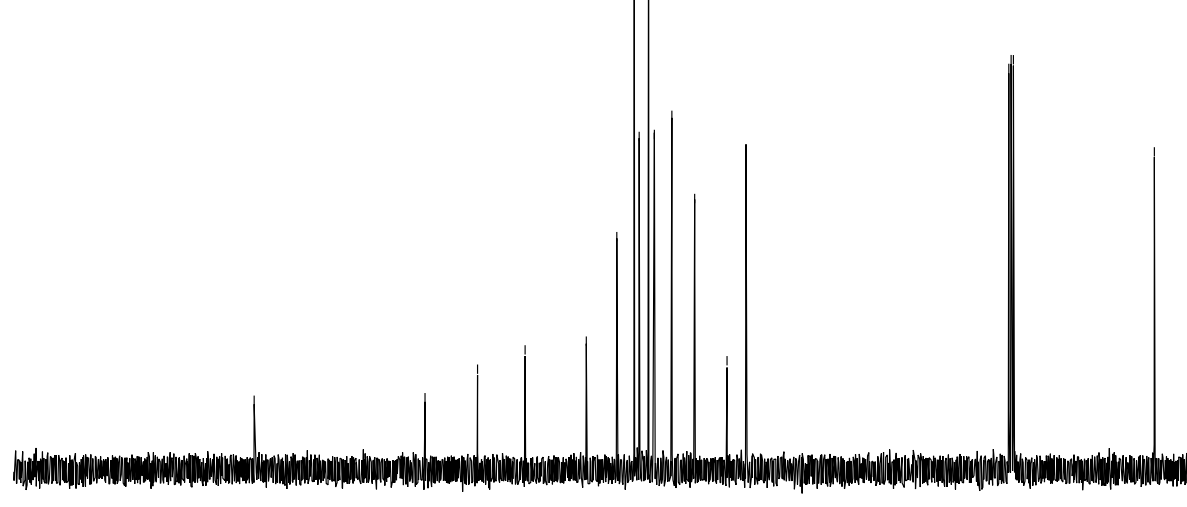

\begin{tabular}{lllllllllllllllllllll}
\hline 210 & 200 & 190 & 180 & 170 & 160 & 150 & 140 & 130 & 120 & $\begin{array}{c}110 \\
\mathrm{f} 1(\mathrm{ppm})\end{array}$ & 90 & 80 & 70 & 60 & 50 & 40 & 30 & 20 & 10 & 0
\end{tabular} 


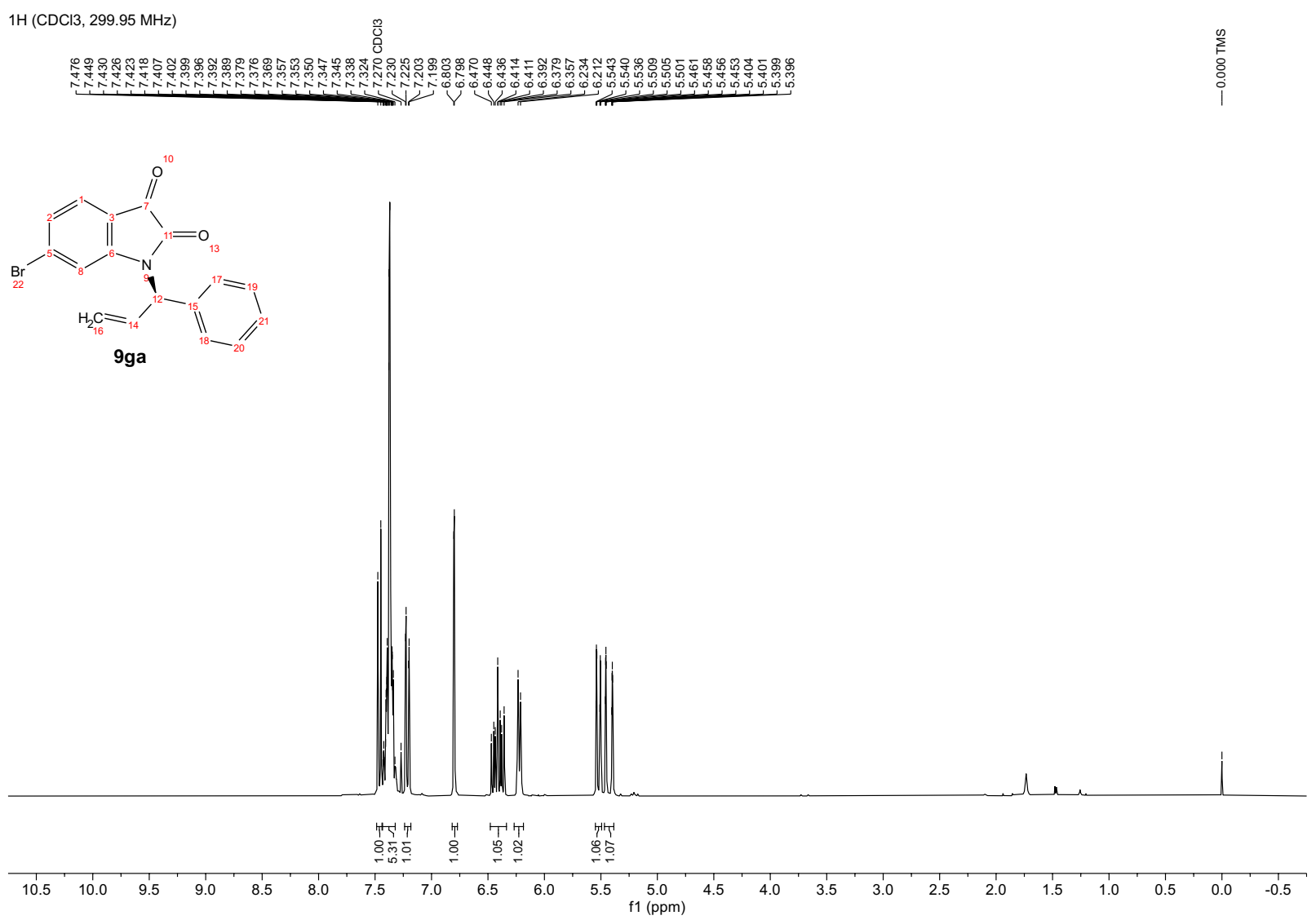

$13 \mathrm{C}(\mathrm{CDCl} 3,75.43 \mathrm{MHz})$
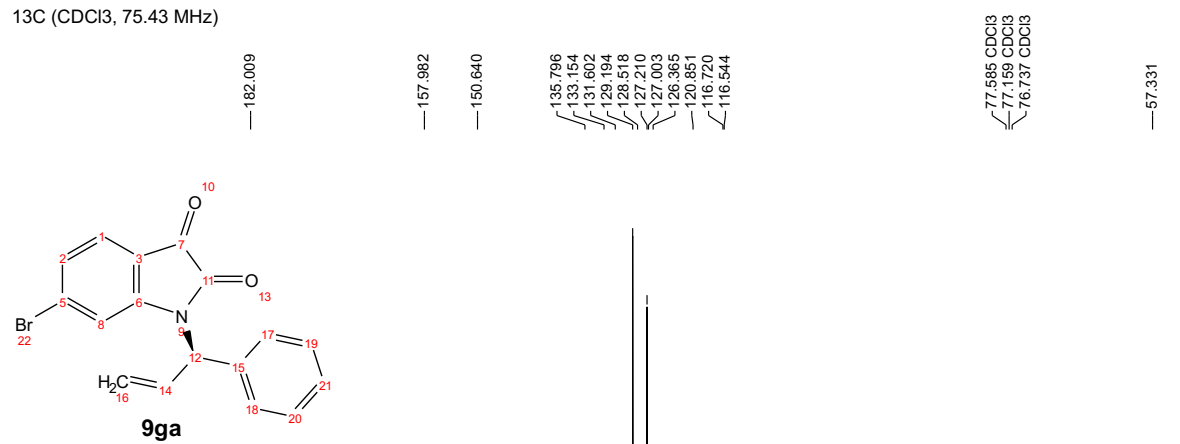

9ga

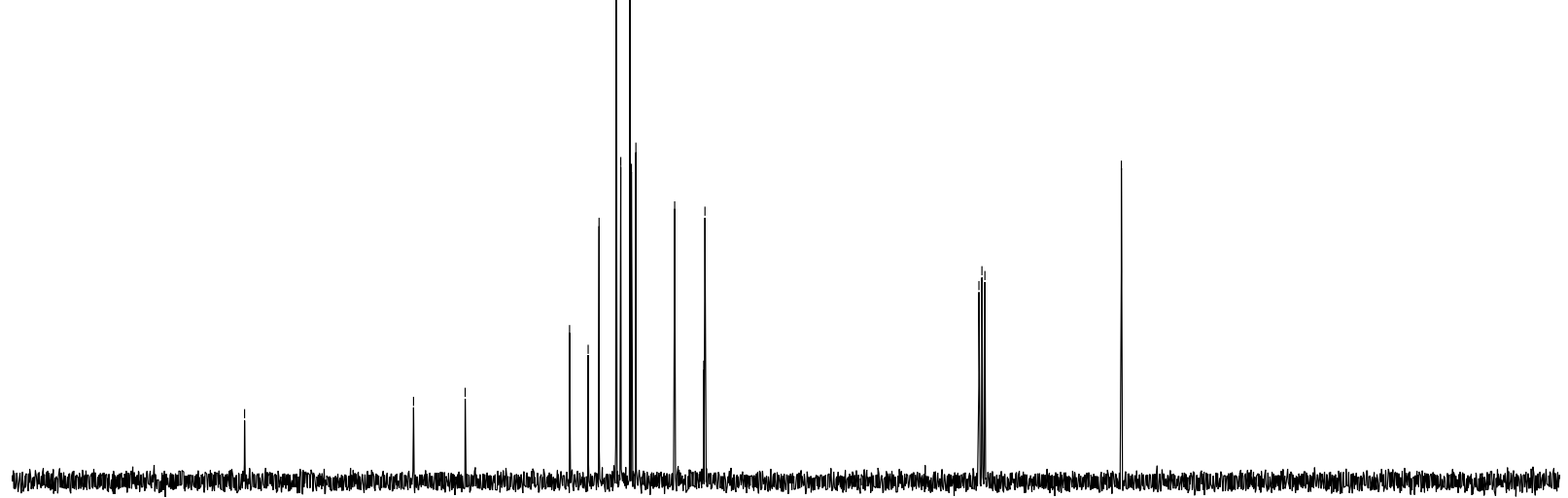

\begin{tabular}{lllllllllllllllllllll}
\hline 210 & 200 & 190 & 180 & 170 & 160 & 150 & 140 & 130 & 120 & $\begin{array}{c}110 \\
\mathrm{f} 1(\mathrm{ppm})\end{array}$ & 90 & 80 & 70 & 60 & 50 & 40 & 30 & 20 & 10 & 0
\end{tabular} 


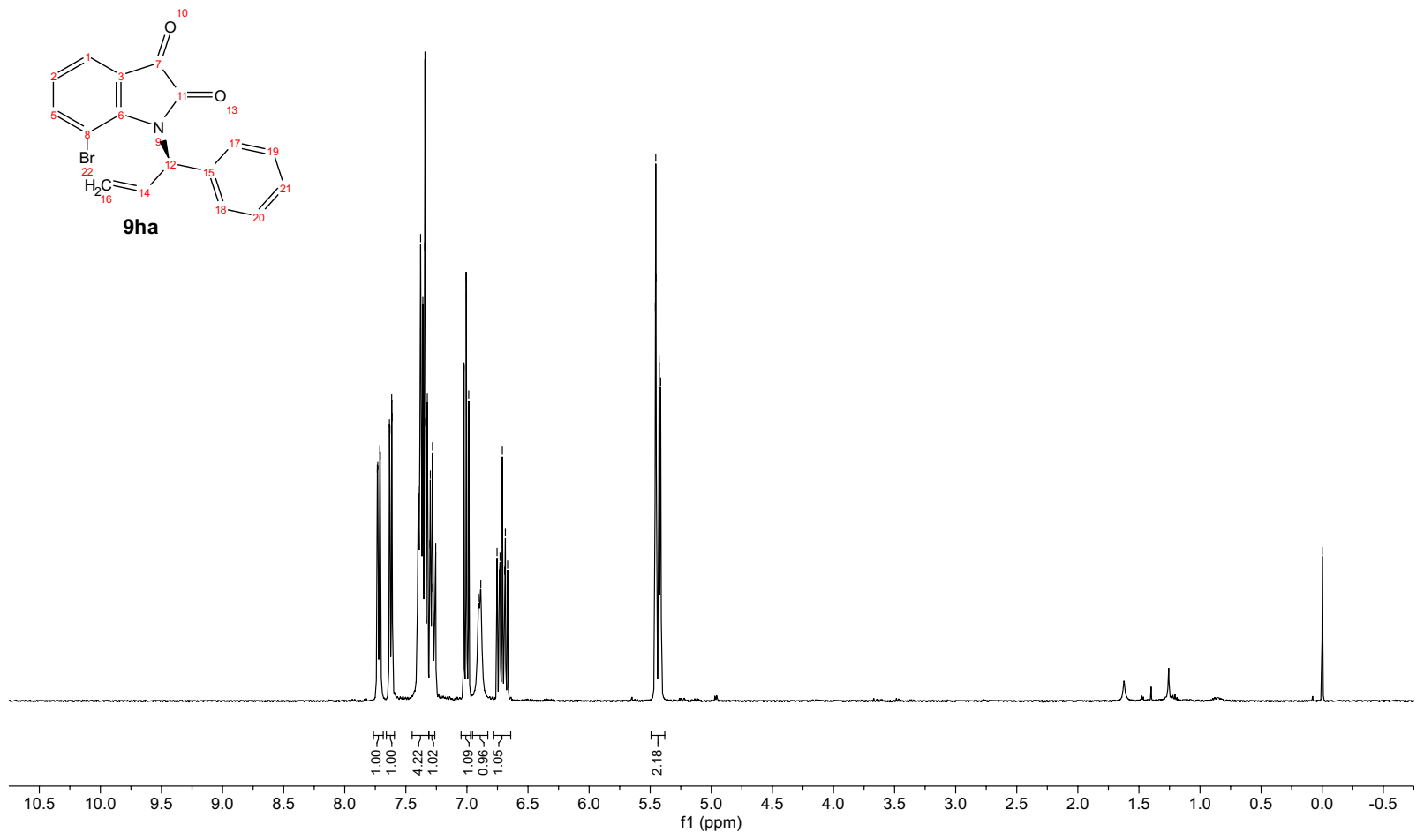

$13 \mathrm{C}(\mathrm{cdcl} 3,100.52 \mathrm{MHz})$

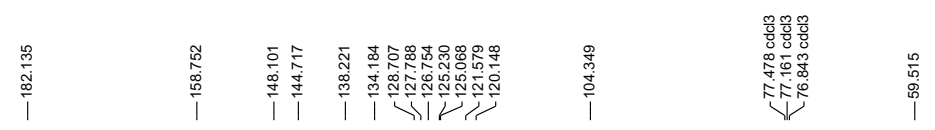

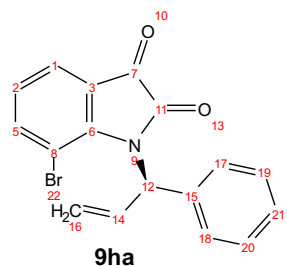

9ha

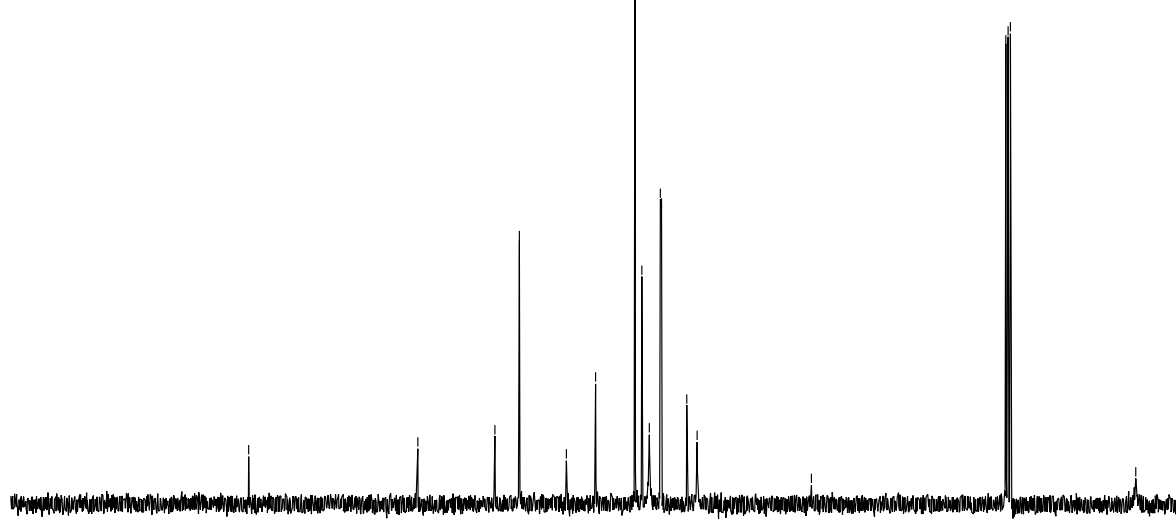

\begin{tabular}{lllllllllllllllllllll}
\hline 210 & 200 & 190 & 180 & 170 & 160 & 150 & 140 & 130 & 120 & $\begin{array}{c}110 \\
\mathrm{f} 1(\mathrm{ppm})\end{array}$ & 90 & 80 & 70 & 60 & 50 & 40 & 30 & 20 & 10 & 0
\end{tabular}




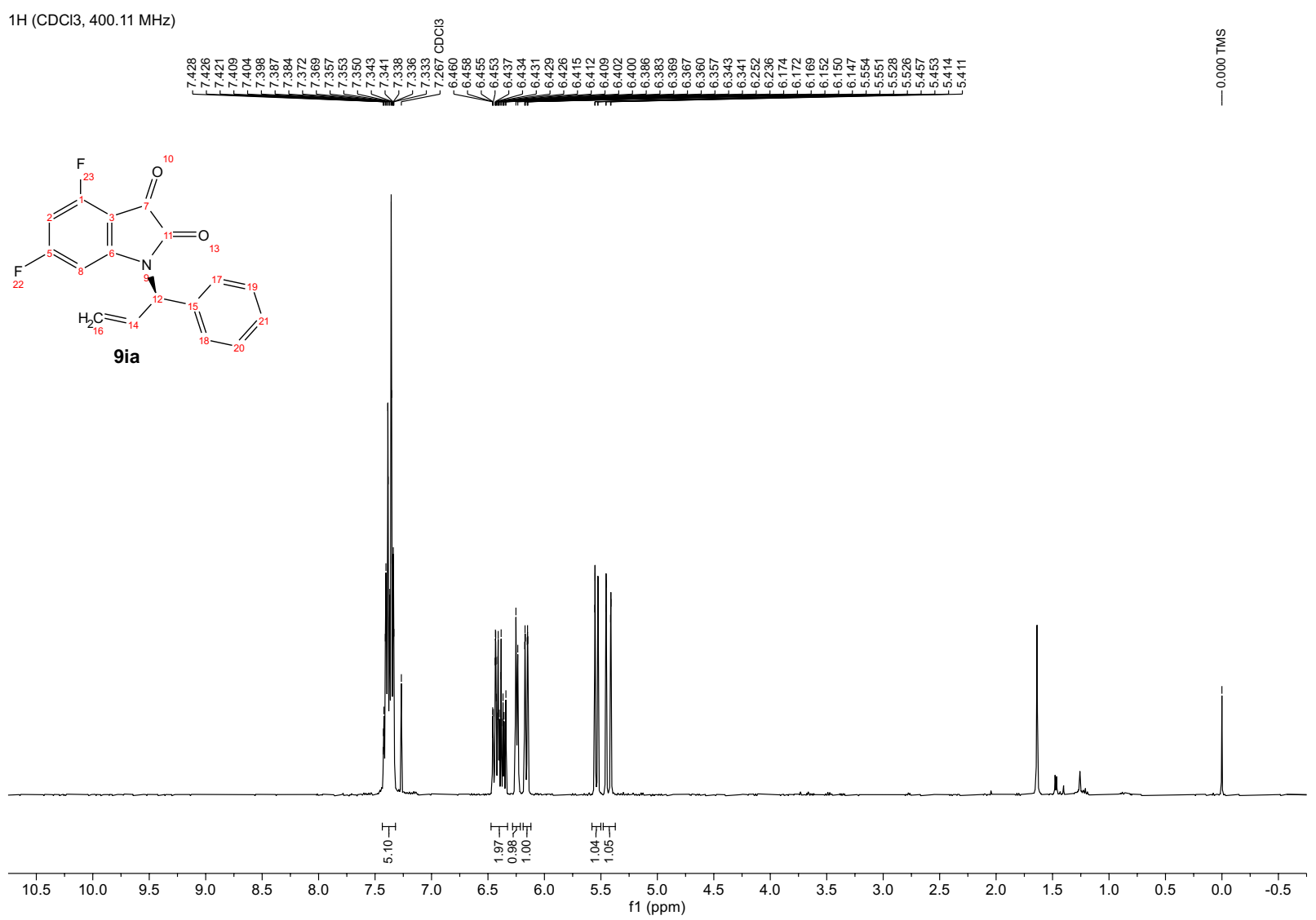

13C (CDCl3, $100.62 \mathrm{MHz})$
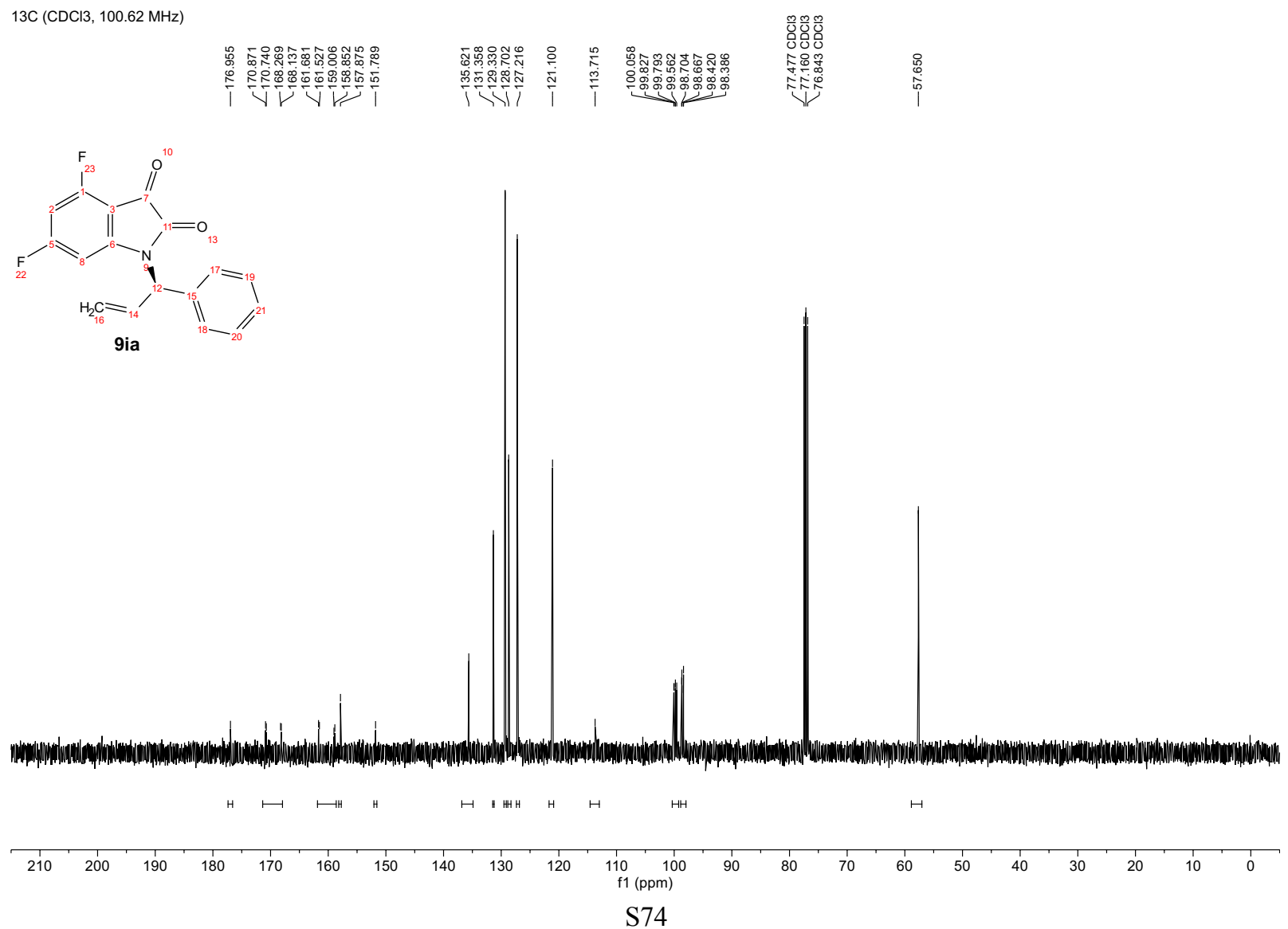
$19 \mathrm{~F}(\mathrm{CDCl} 3,376.44 \mathrm{MHz})$

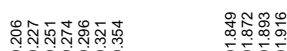

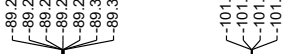

9ia

$\longmapsto$

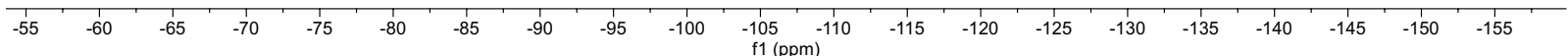




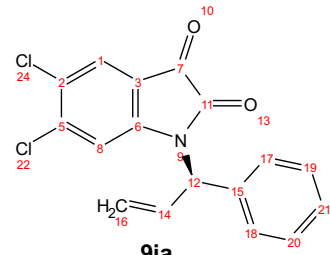

9ja

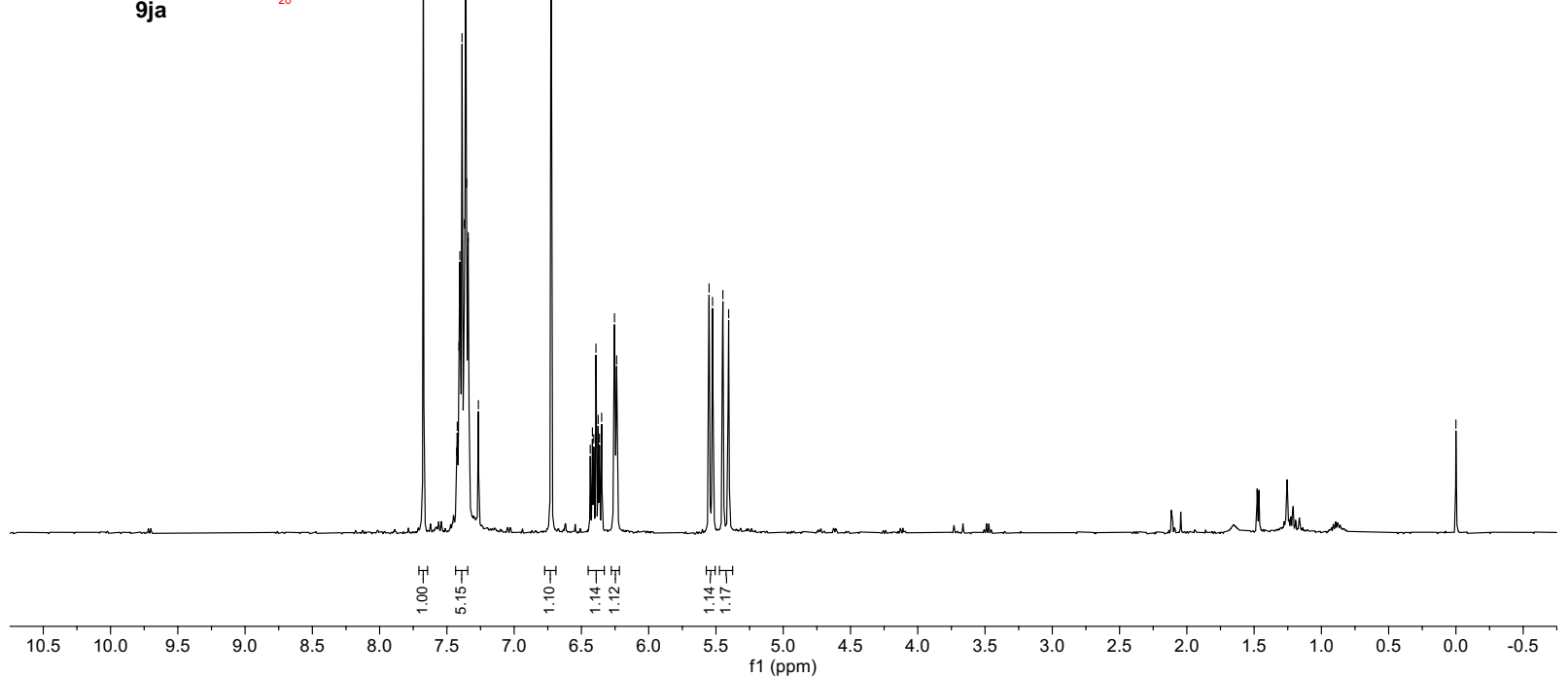

13C (cdcl3, $100.52 \mathrm{MHz})$

广

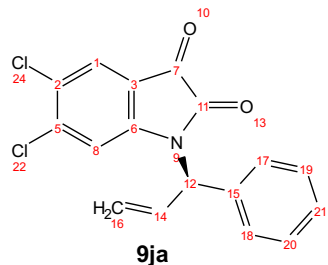

9ja

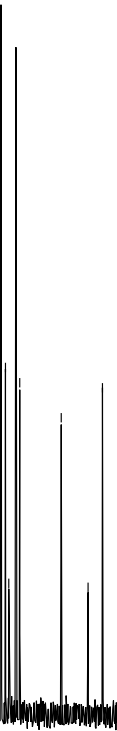

Nin

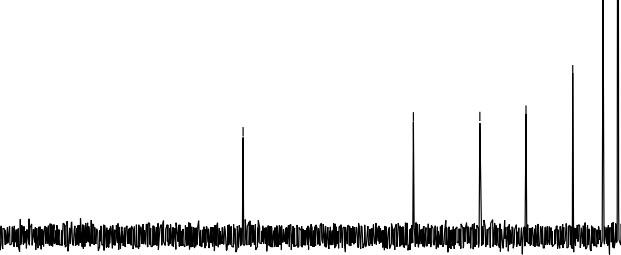

\begin{tabular}{lllllllllll}
\hline 210 & 200 & 190 & 180 & 170 & 160 & 150 & 140 & 130 & 120 & $\begin{array}{c}110 \\
\mathrm{f} 1(\mathrm{ppm})\end{array}$
\end{tabular} 
<smiles></smiles>

9ka

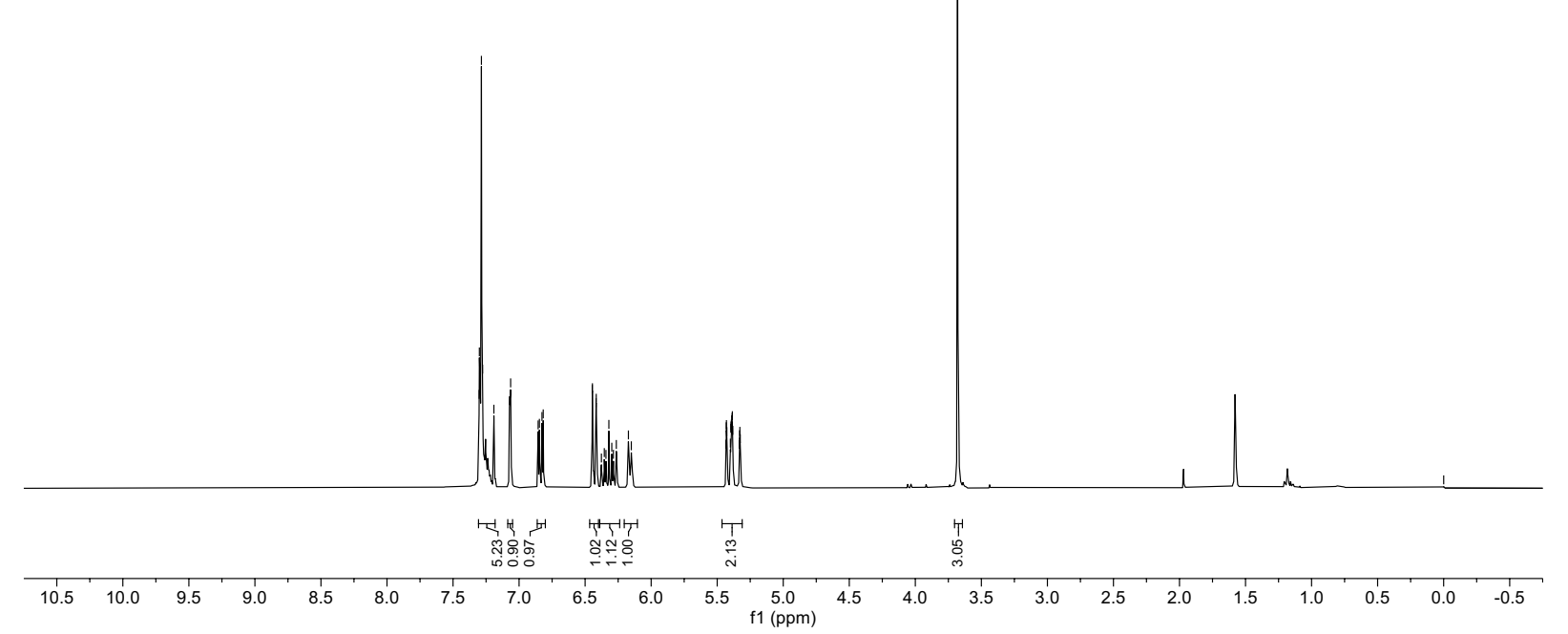

$13 \mathrm{C}(\mathrm{cdcl} 3,100.52 \mathrm{MHz})$

\begin{tabular}{|c|c|c|c|c|}
\hline 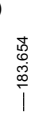 & 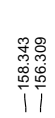 & 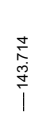 & 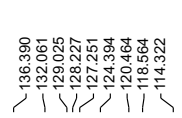 & $\begin{array}{l}\bar{g} \\
\text { o }\end{array}$ \\
\hline
\end{tabular}
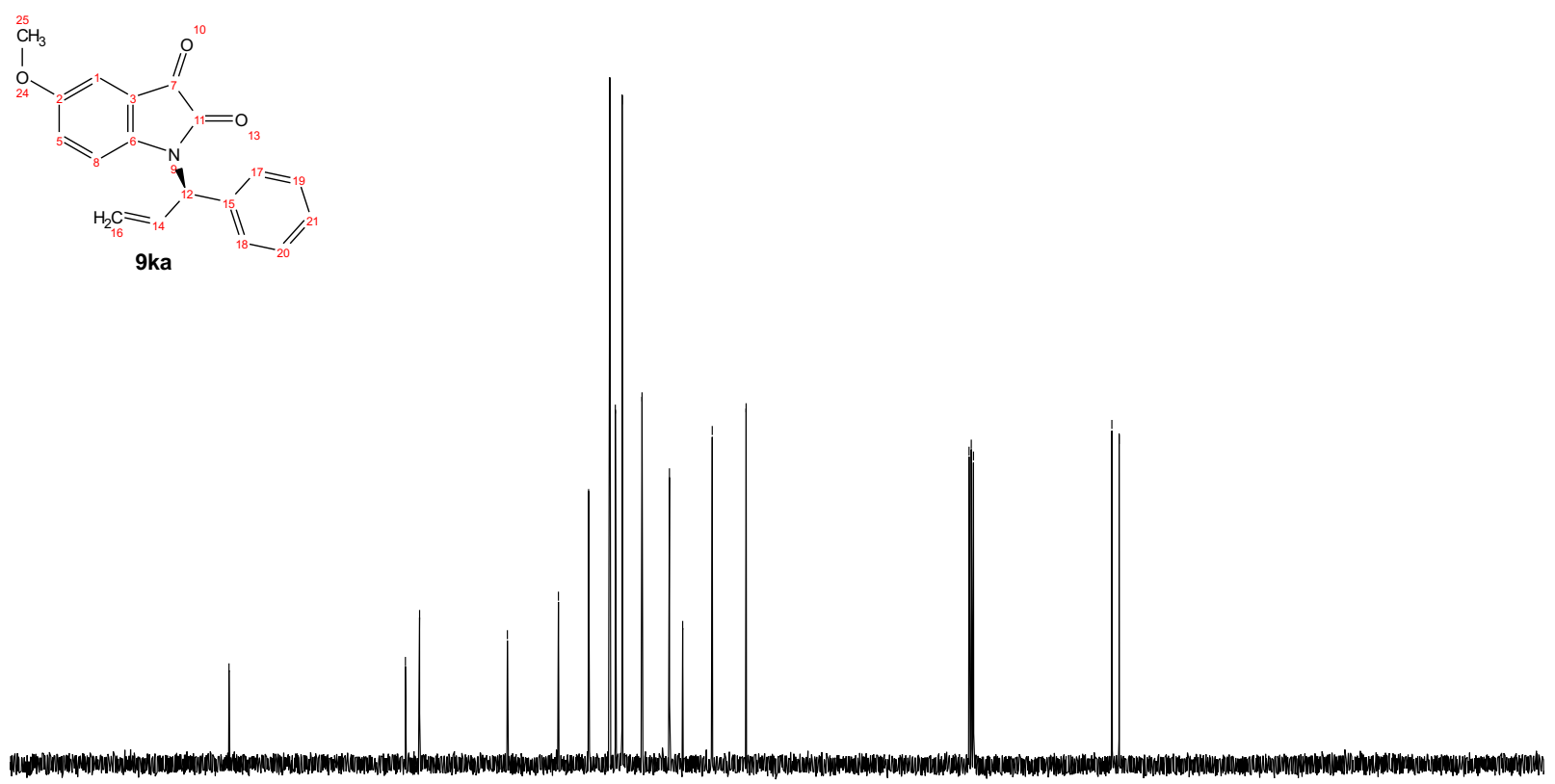

\begin{tabular}{llllllllllllllllllllll}
\hline 210 & 200 & 190 & 180 & 170 & 160 & 150 & 140 & 130 & 120 & $\begin{array}{c}110 \\
\mathrm{f} 1(\mathrm{ppm})\end{array}$ & 90 & 80 & 70 & 60 & 50 & 40 & 30 & 20 & 10 & 0
\end{tabular} 

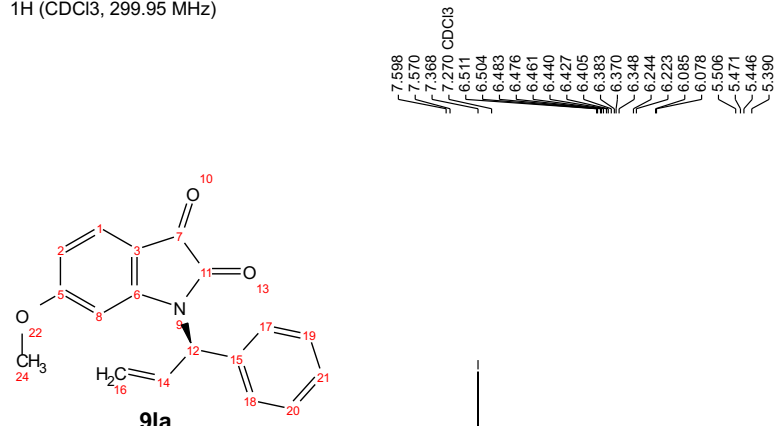

9la

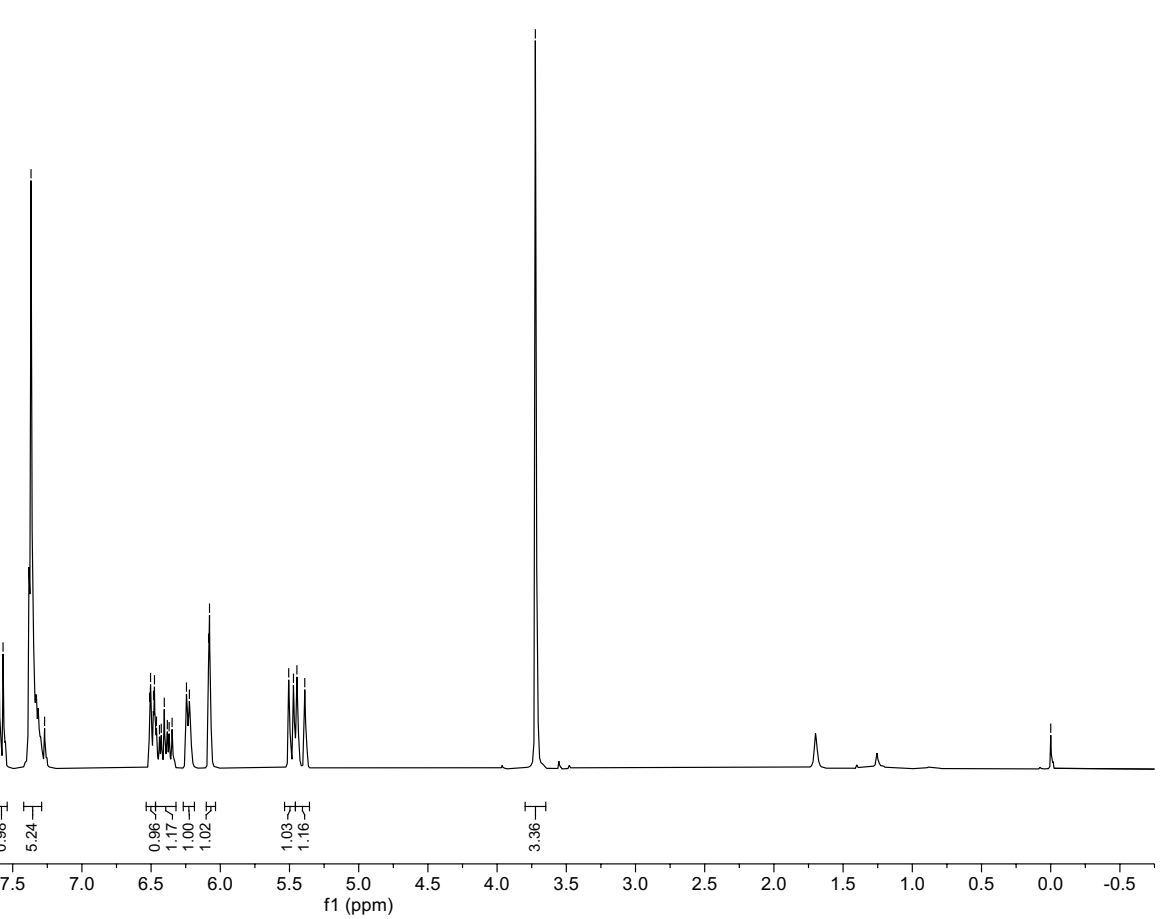

13C (cdcl3, $100.52 \mathrm{MHz}$ )

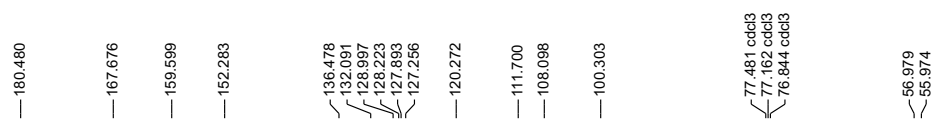<smiles>[13CH3]Oc1ccc2c(c1)N(P(c1cnccn1)c1nccnn1)C(=O)C2=O</smiles>

9la

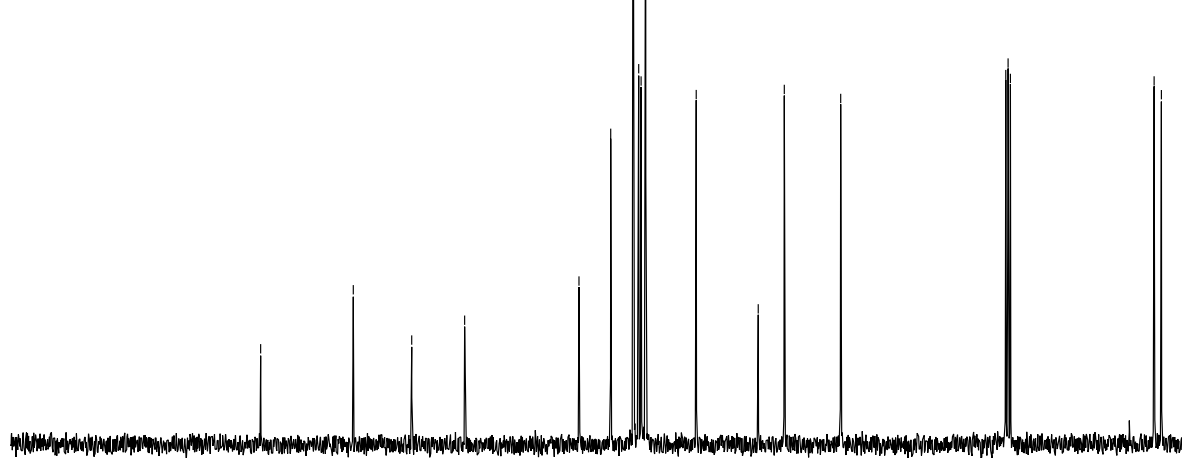

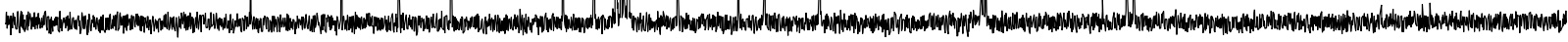

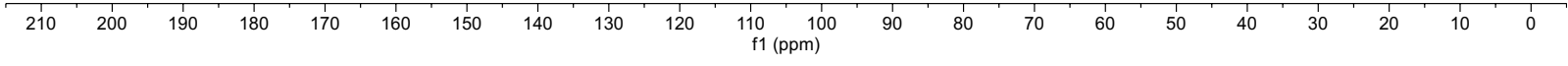


$1 \mathrm{H}(\mathrm{CDCl} 3,299.95 \mathrm{MHz})$

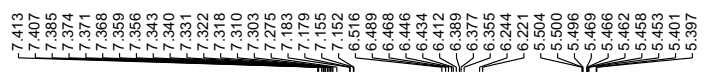

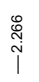

2
$\vdots$
$\vdots$
0
0
0
0
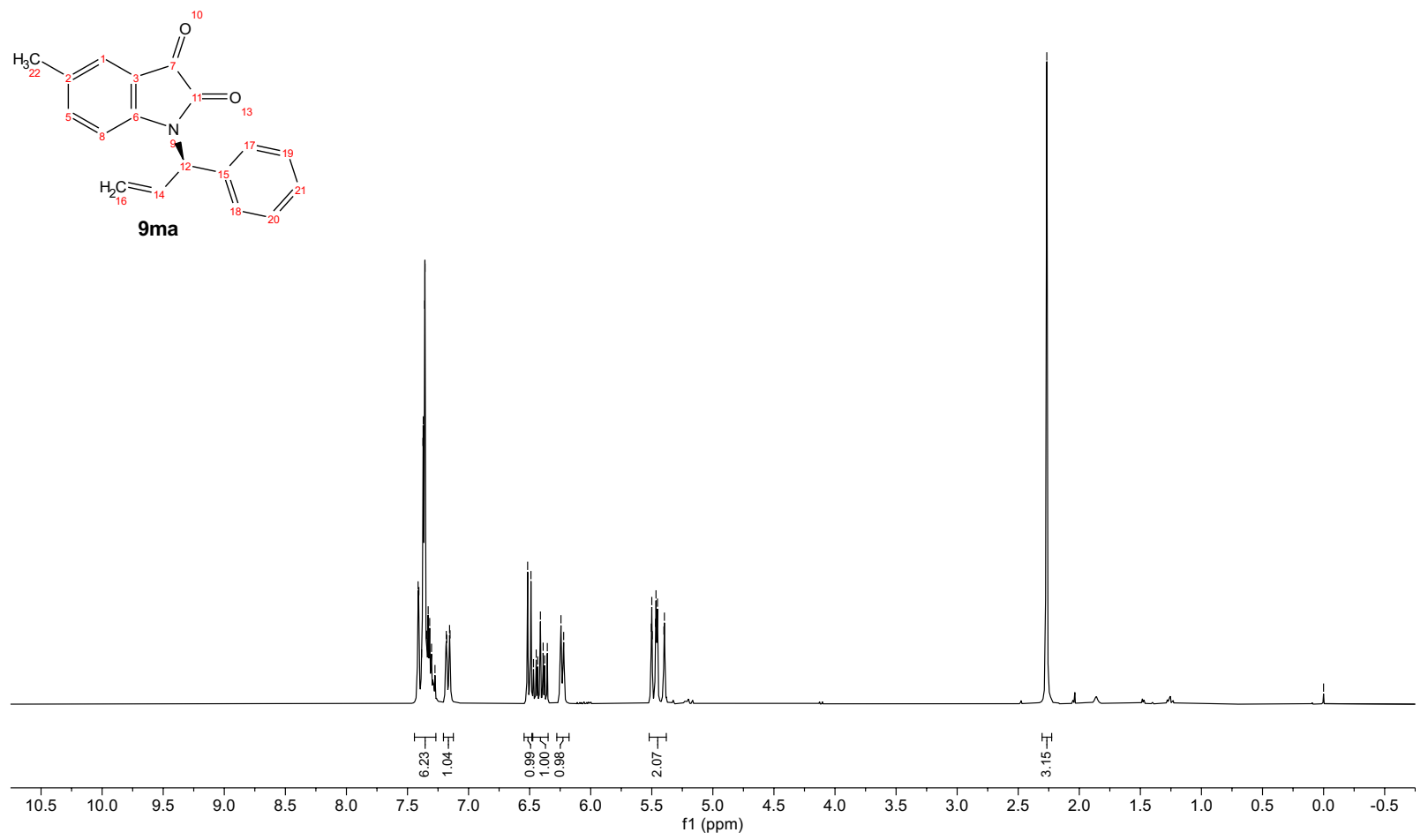

$13 \mathrm{C}(\mathrm{CDCl} 3,100.62 \mathrm{MHz})$

章
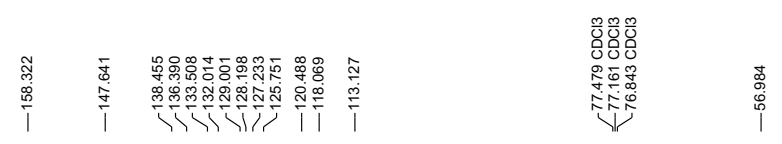

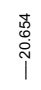
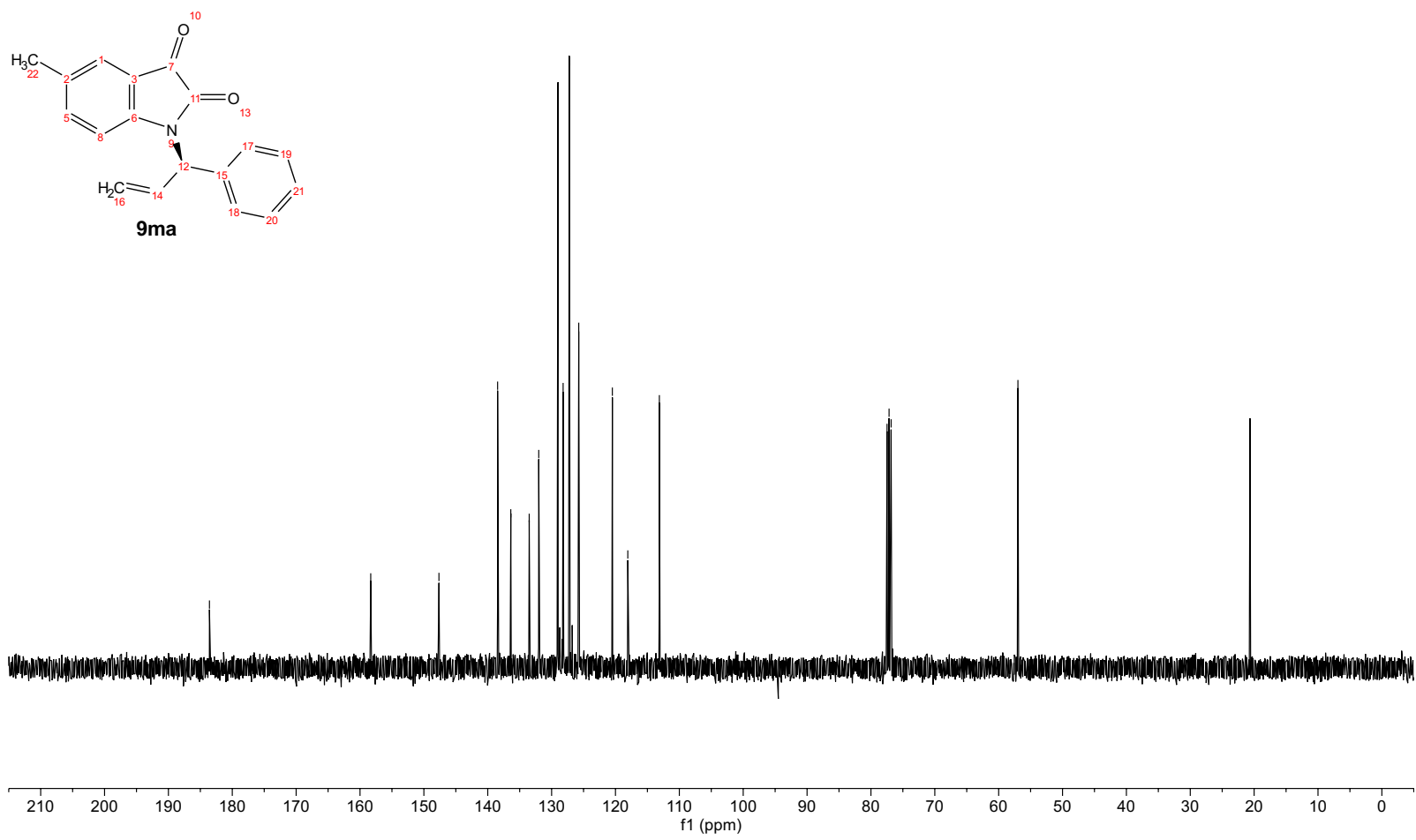

S79 
1H (CDCl3, 400.11 MHz)

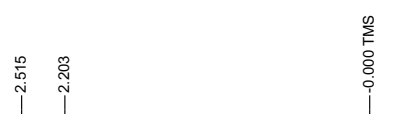<smiles></smiles>

9na

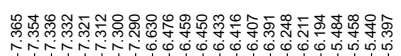

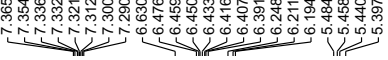

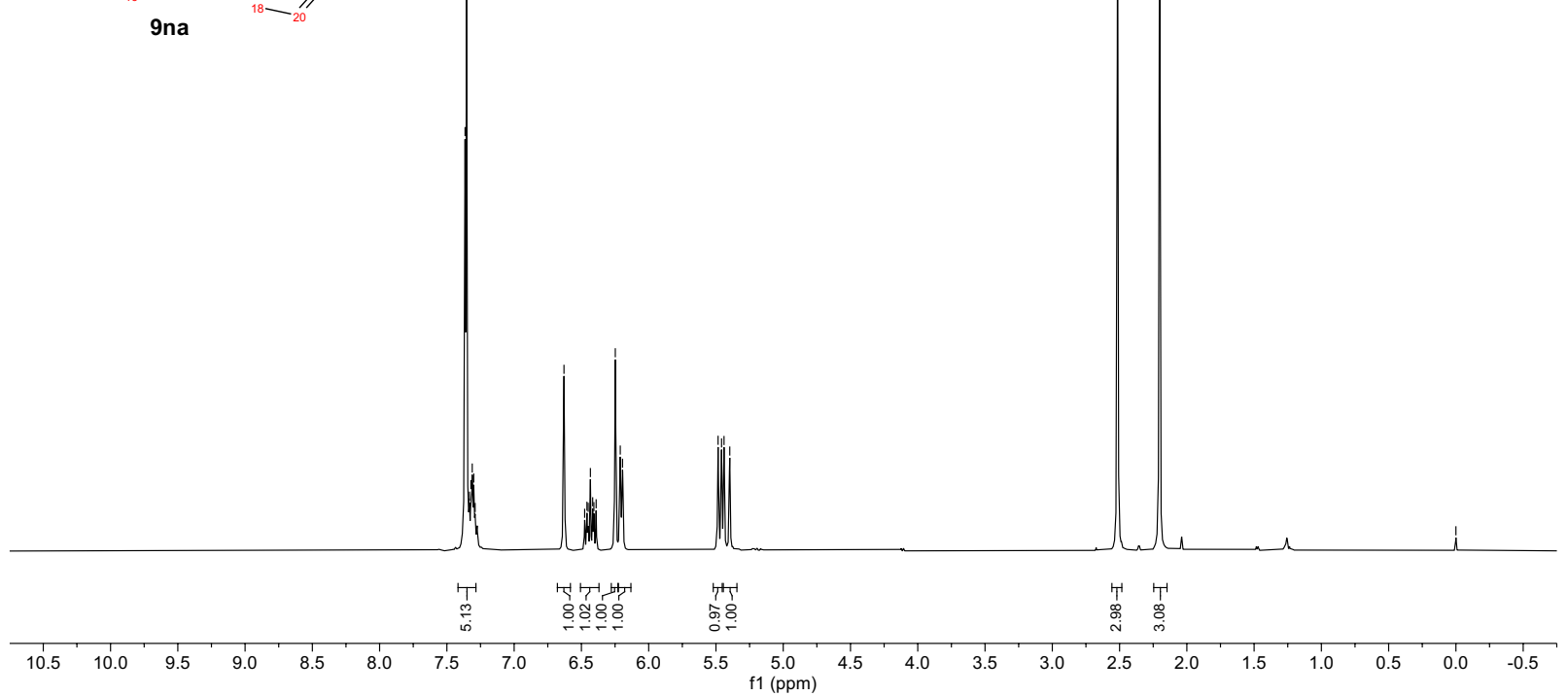

13C (CDCl3, $100.62 \mathrm{MHz})$

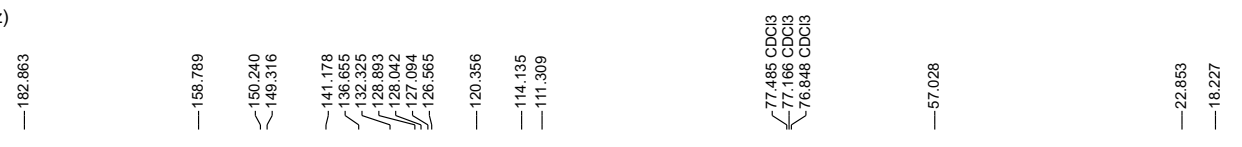<smiles>C=CN(c1cccnc1)N1C(=O)C(=O)c2c(O)cc(C)cc21</smiles>

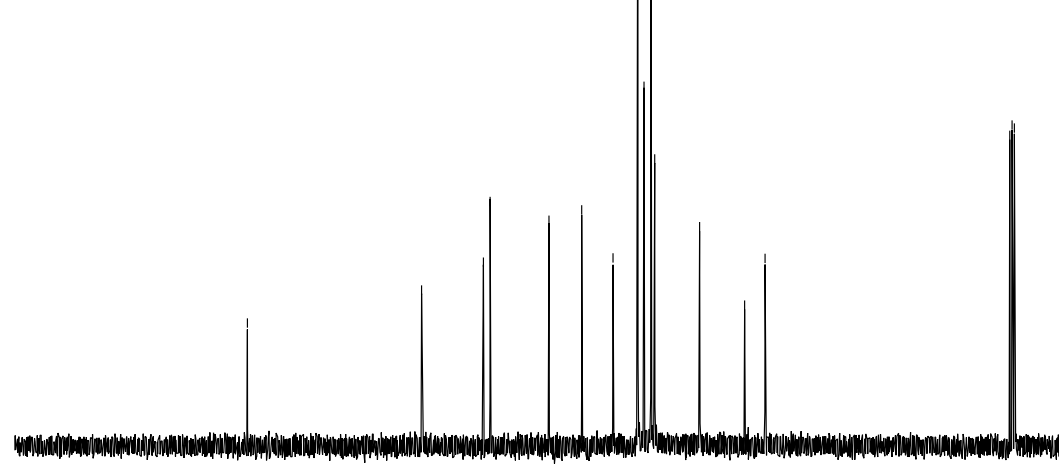

\begin{tabular}{llllllllllllllllllllll}
\hline 210 & 200 & 190 & 180 & 170 & 160 & 150 & 140 & 130 & 120 & $\begin{array}{c}110 \\
\mathrm{f} 1(\mathrm{ppm})\end{array}$ & 90 & 80 & 70 & 60 & 50 & 40 & 30 & 20 & 10 & 0
\end{tabular} 


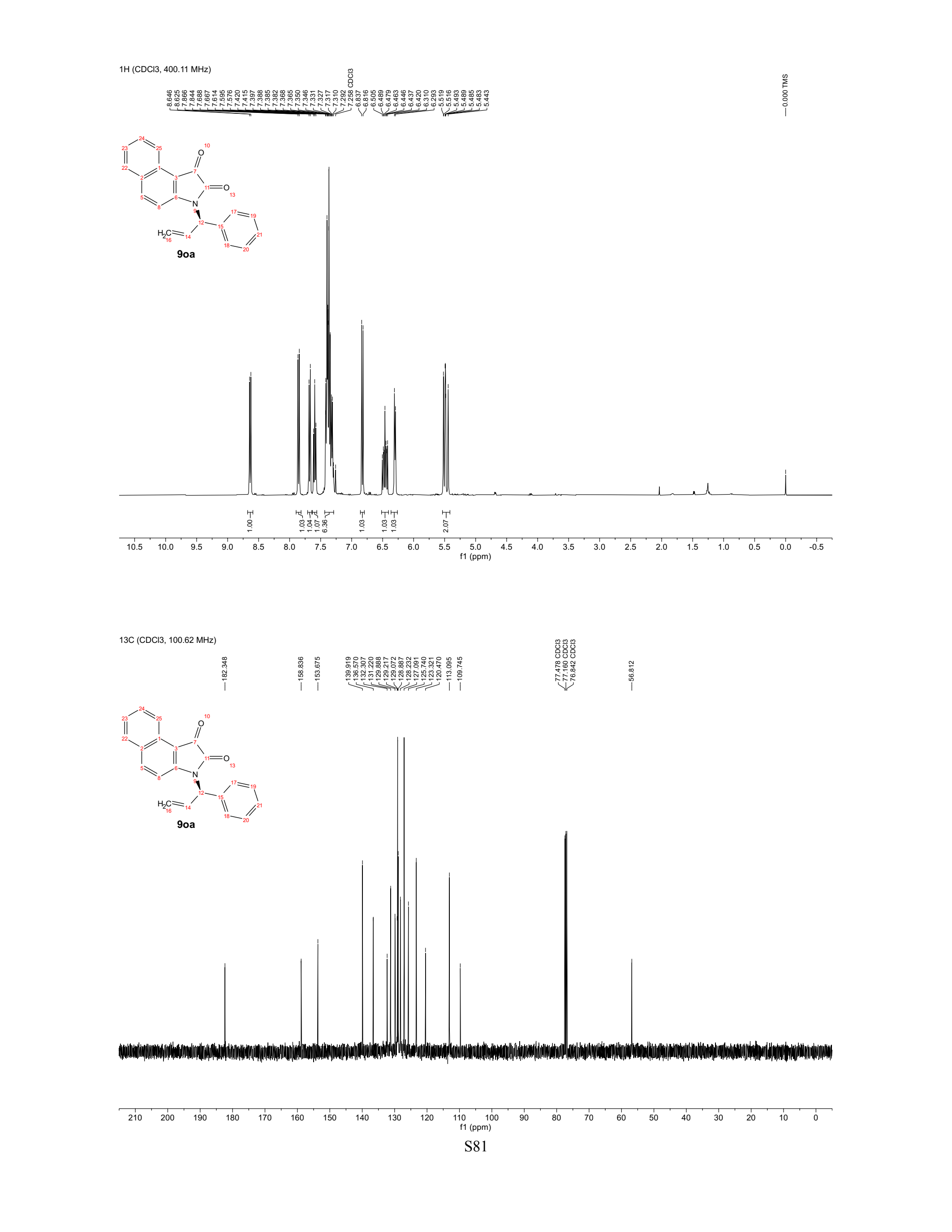



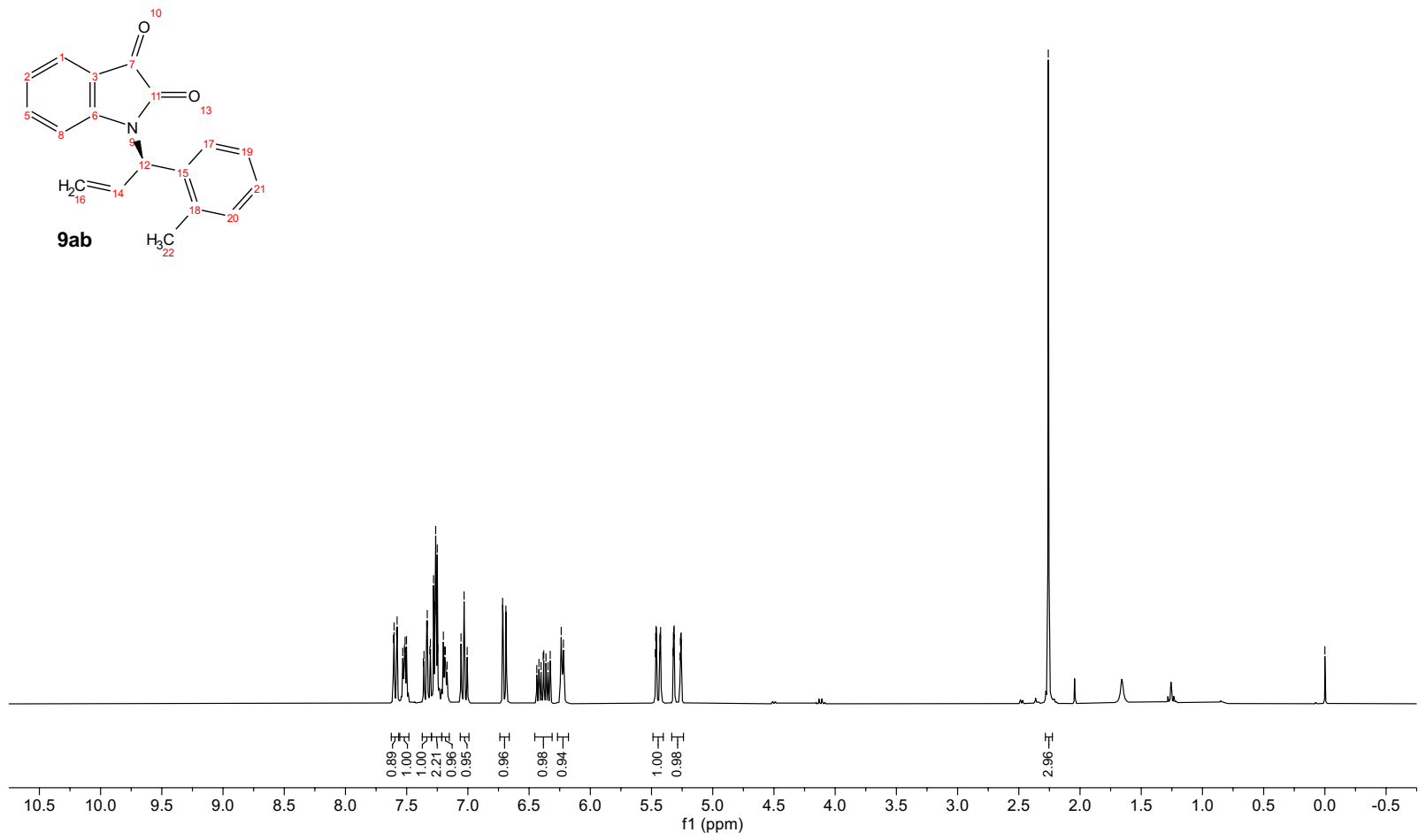

13C (cdcl3, $100.52 \mathrm{MHz}$ )

$$
\text { | }
$$
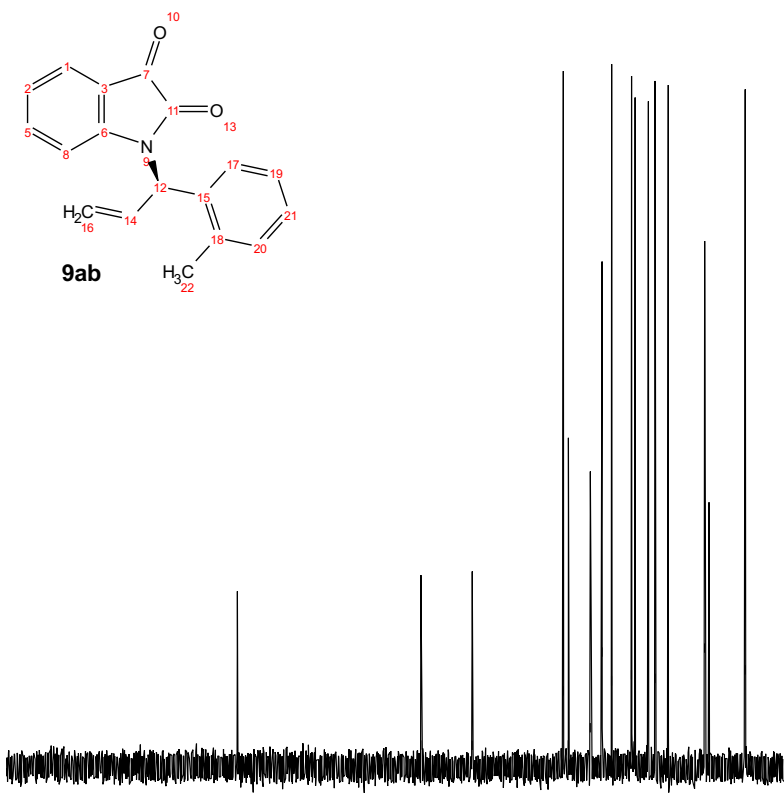

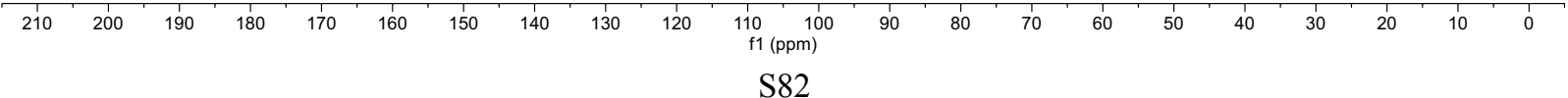



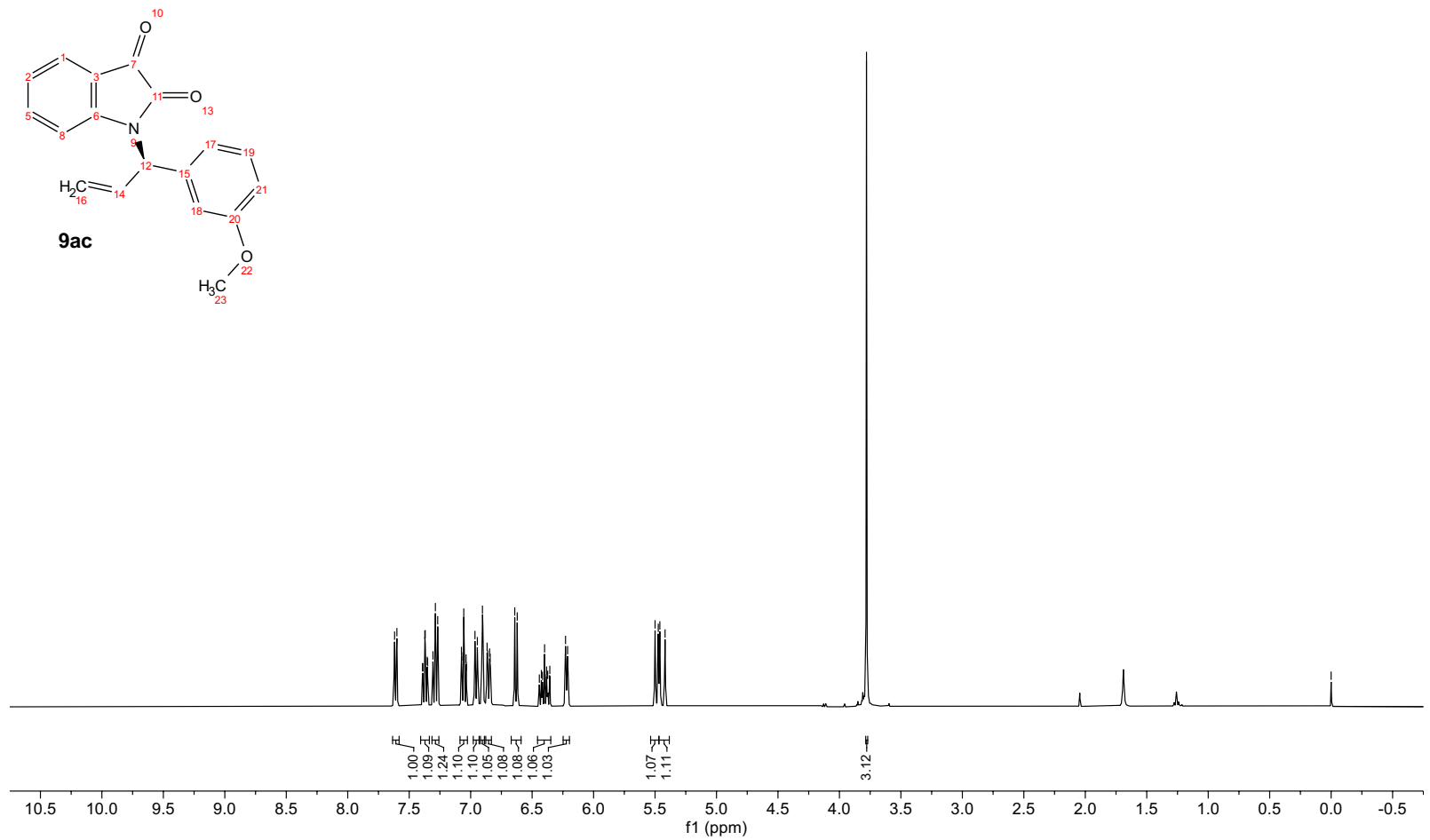

$13 \mathrm{C}(\mathrm{cdcl} 3,100.52 \mathrm{MHz})$

\begin{tabular}{|c|c|c|c|}
\hline & 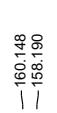 & 兽 & 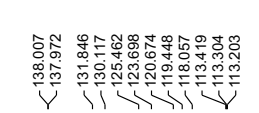 \\
\hline
\end{tabular}<smiles></smiles>

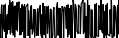

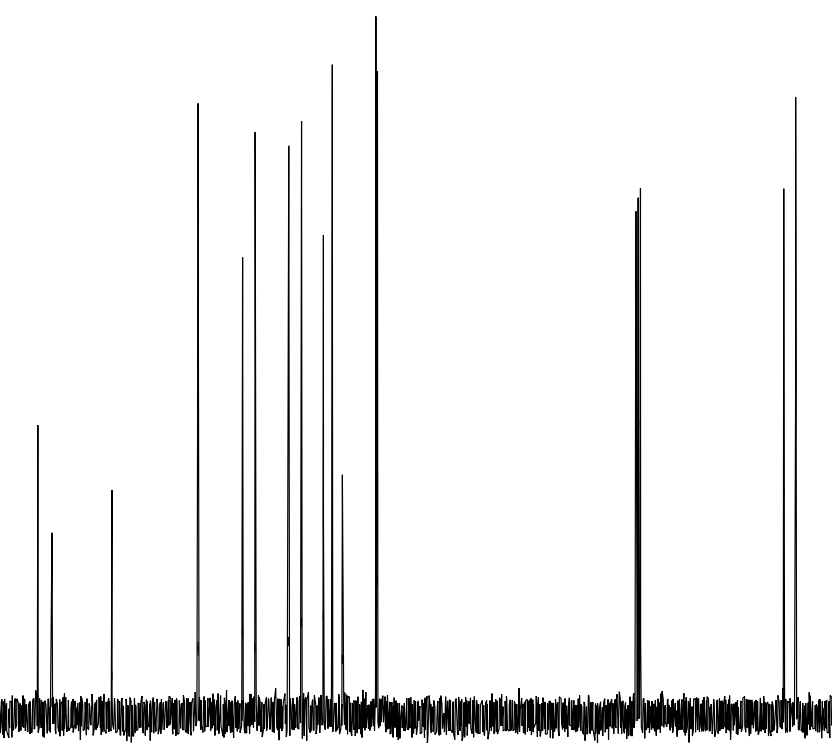

\begin{tabular}{lllllllllllllllllllllll}
\hline & 200 & 190 & 180 & 170 & 160 & 150 & 140 & 130 & 120 & $\begin{array}{c}1 \\
\mathrm{f} 1\end{array}(\mathrm{ppm})$ & 100 & 90 & 80 & 70 & 60 & 50 & 40 & 30 & 20 & 10 & 0
\end{tabular}



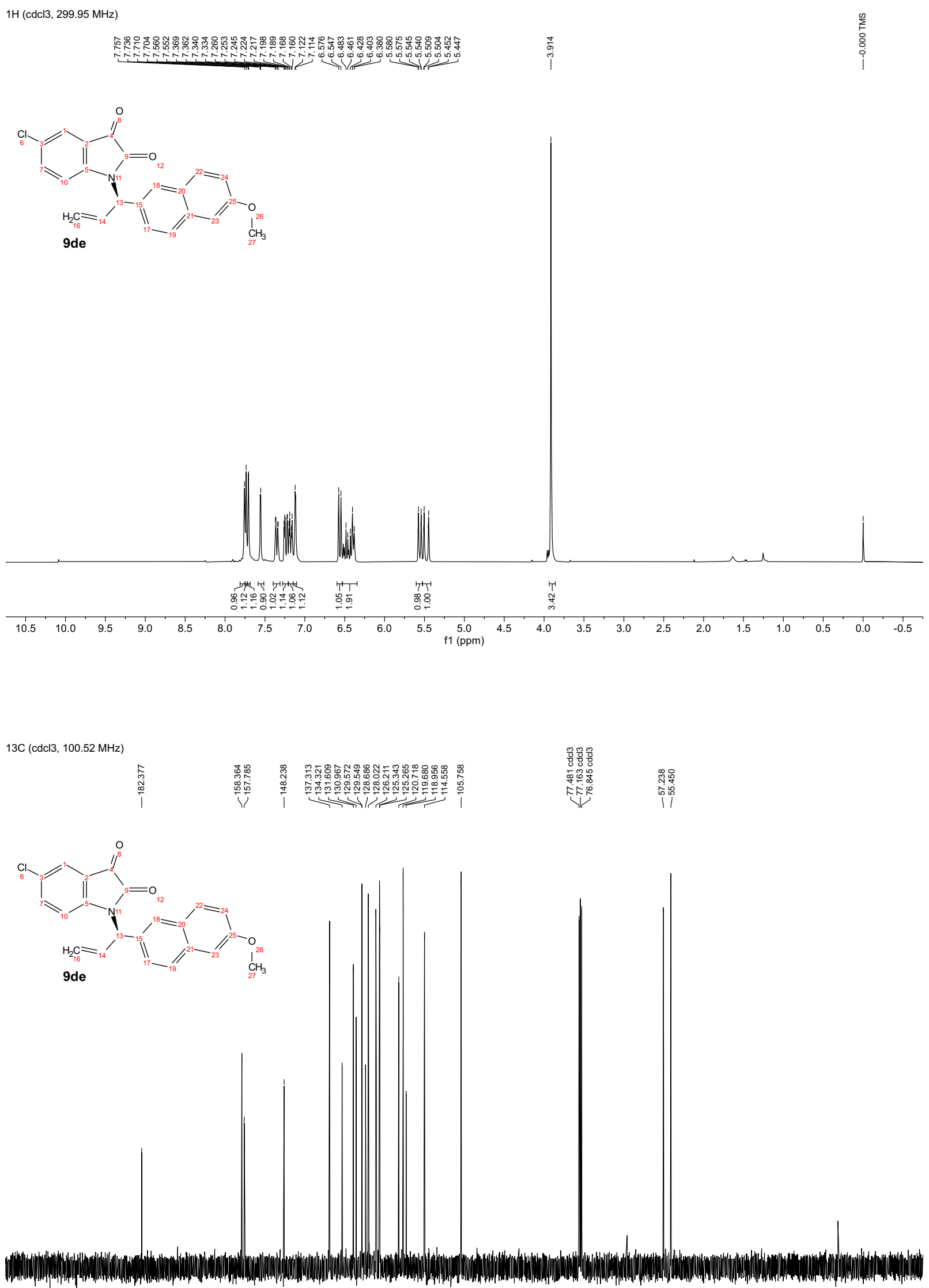

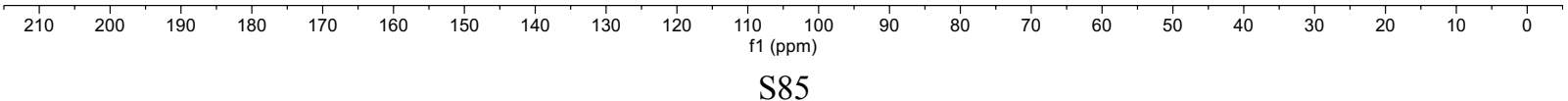



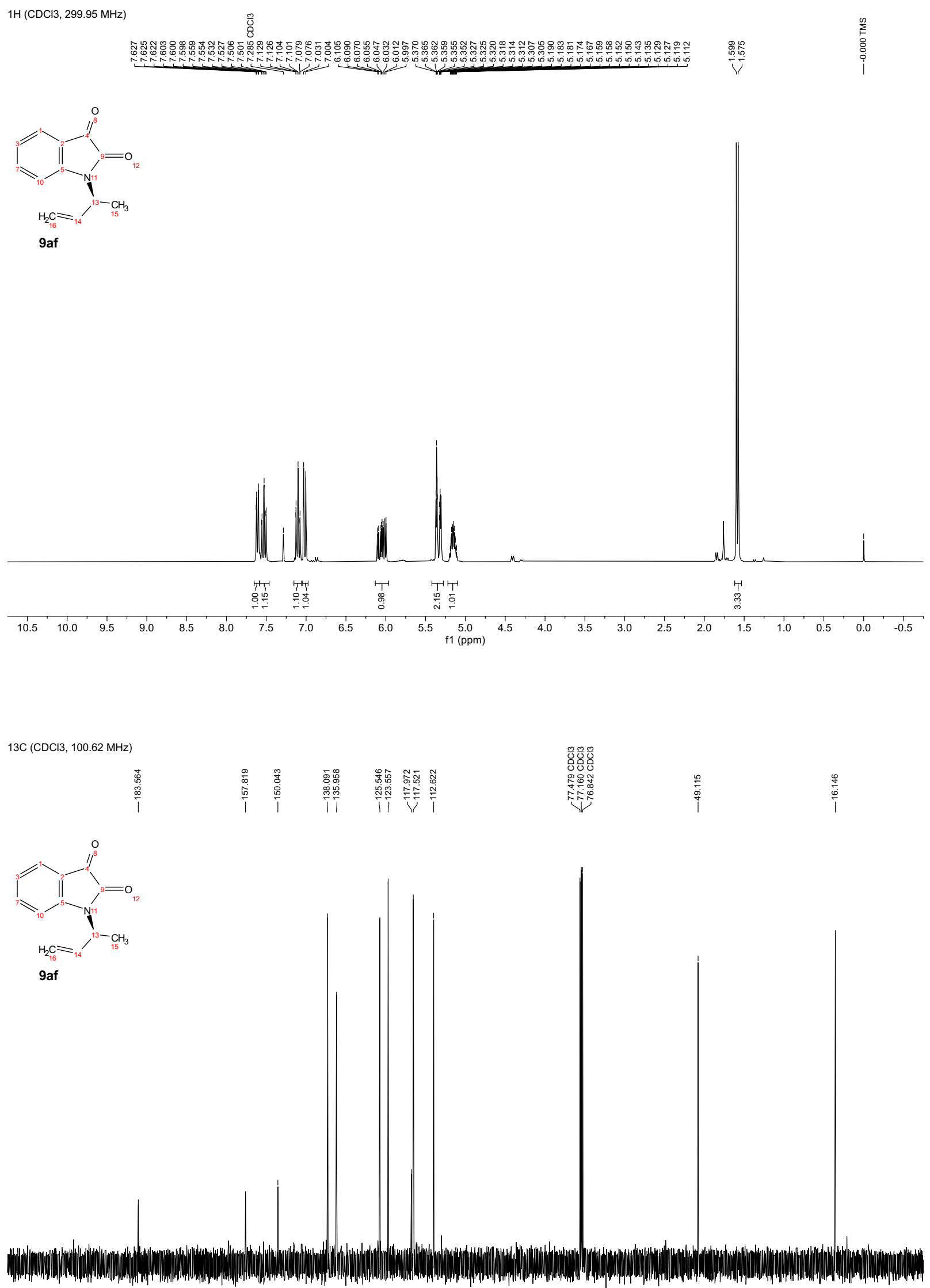

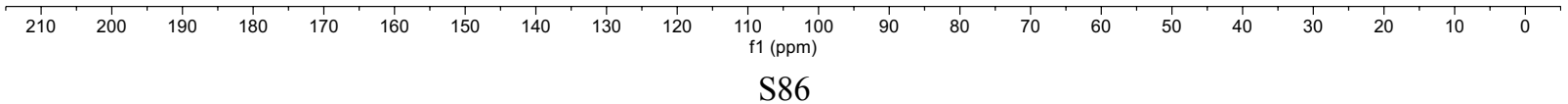




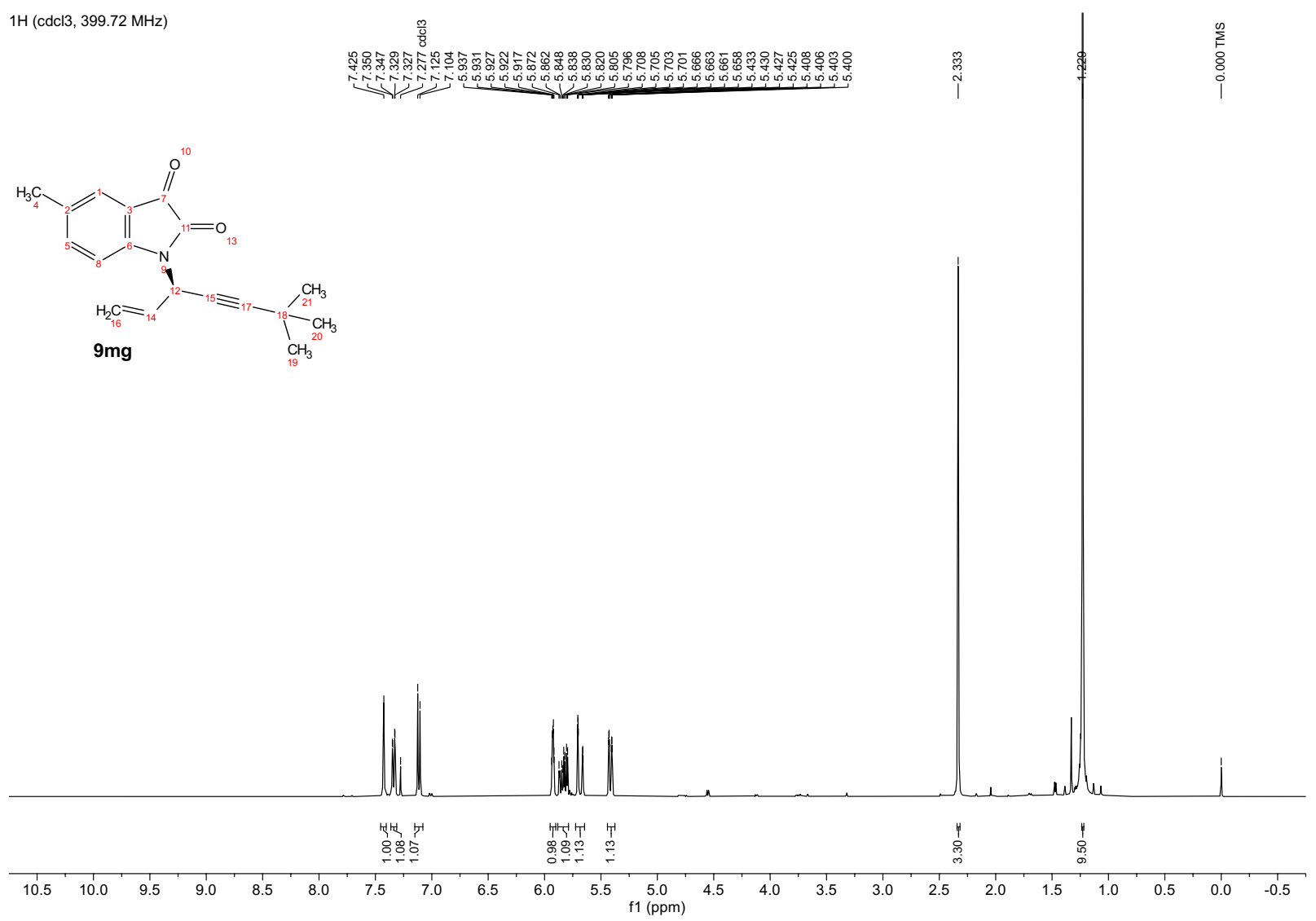

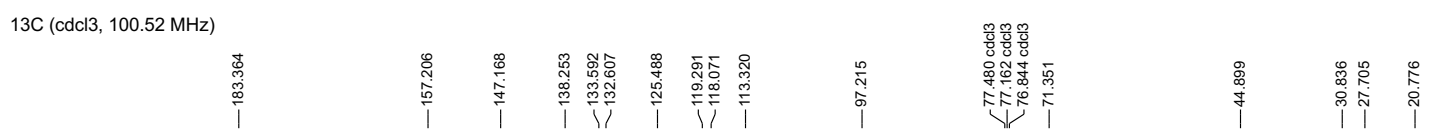
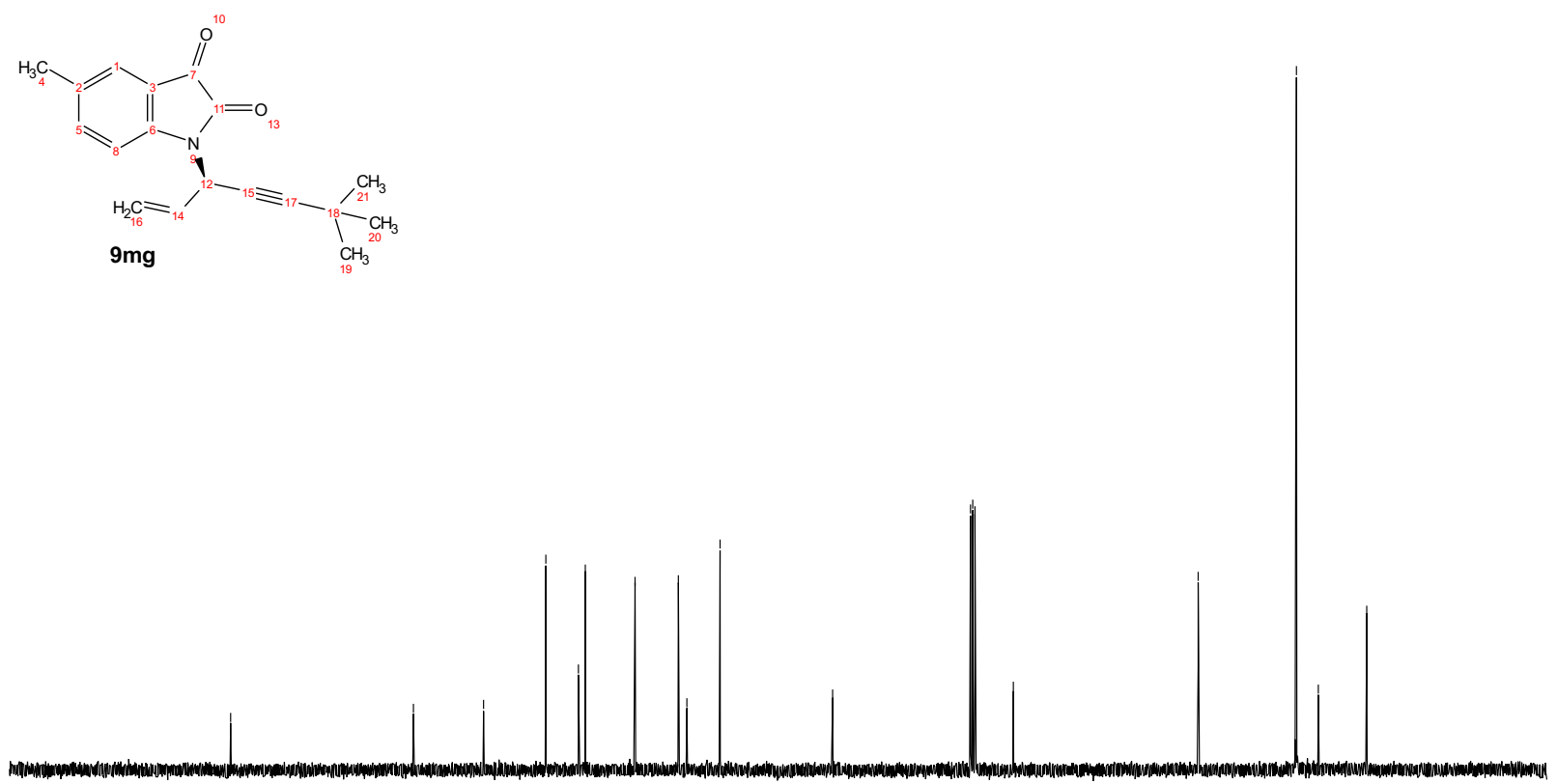

\begin{tabular}{llllllllllllllllllllllllll}
\hline 10 & 200 & 190 & 180 & 170 & 160 & 150 & 140 & 130 & 120 & $\begin{array}{c}110 \\
\mathrm{f} 1(\mathrm{ppm})\end{array}$ & 90 & 80 & 70 & 60 & 50 & 40 & 30 & 20 & 10 & 0
\end{tabular} 

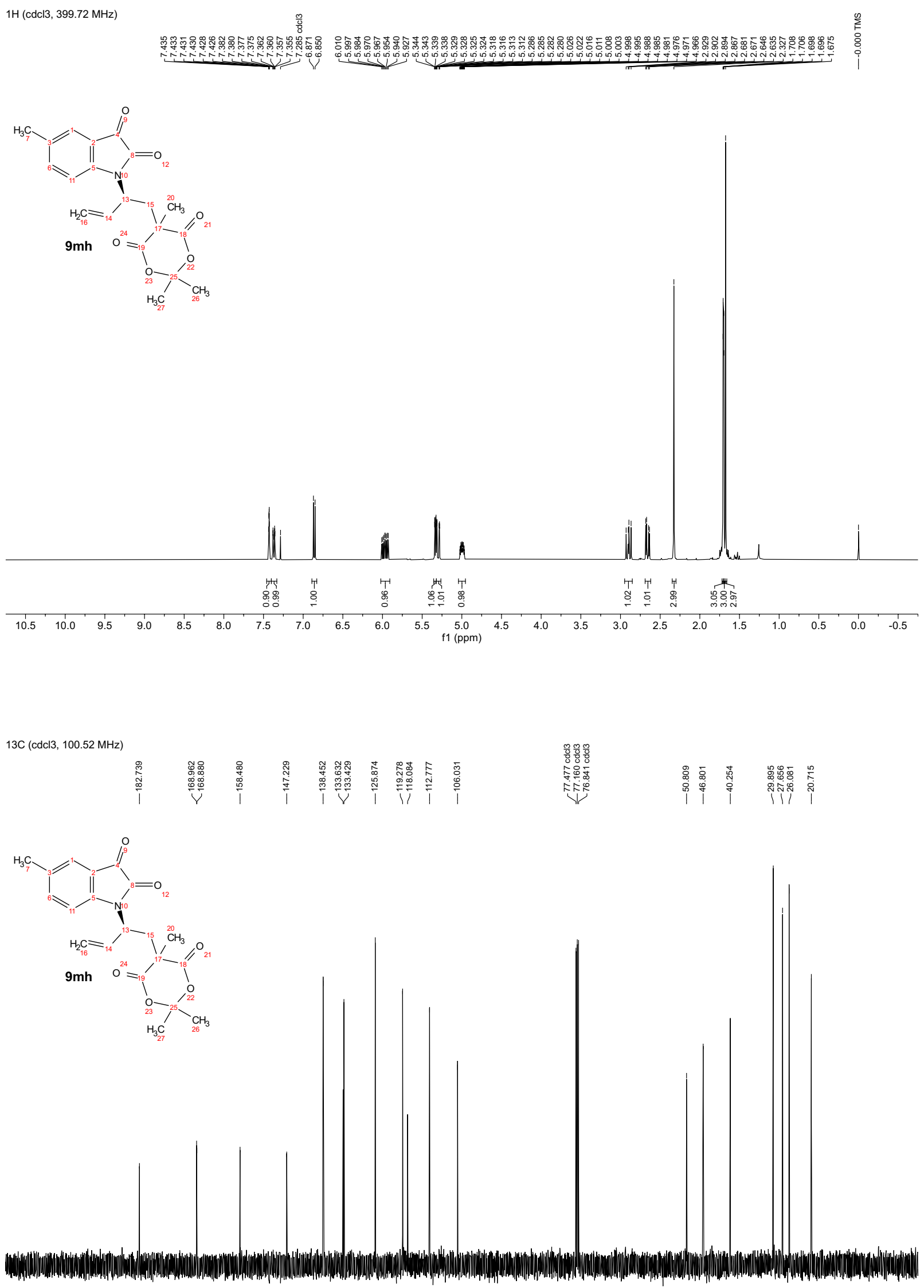

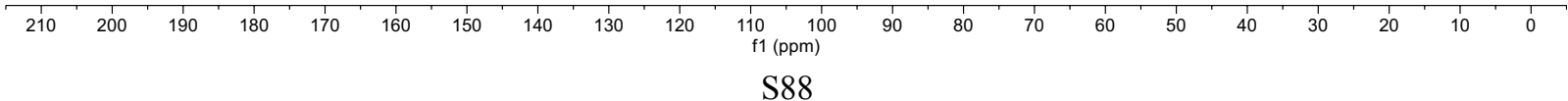


<smiles>C=CN(NOC(=O)C(C)(C)C)N1C(=O)C(=O)c2cc(I)cnc21</smiles>

9ei

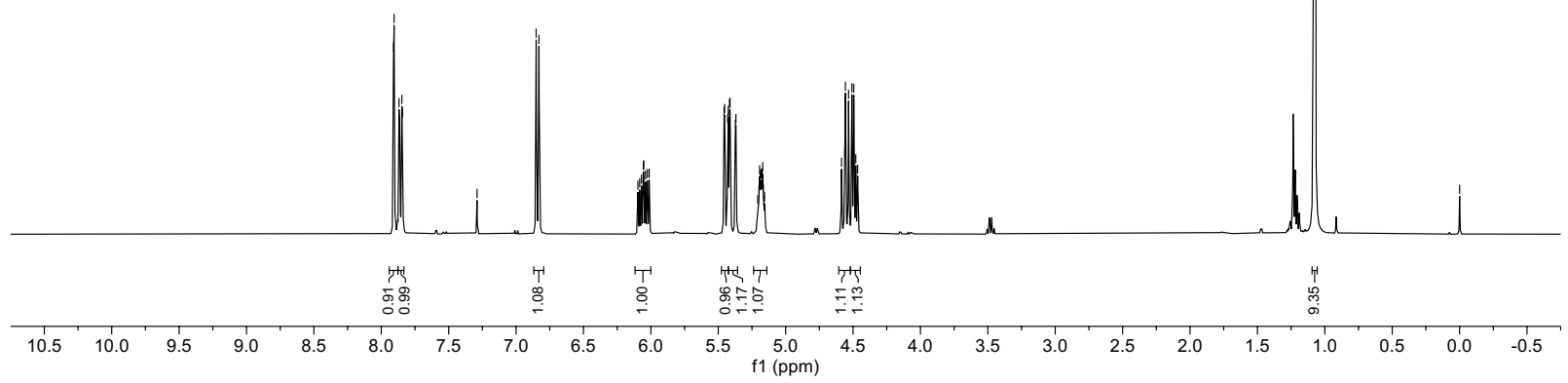

13C (cdcl3, 100.52 MHz)

至<smiles>C=CN(NOC(=O)C(C)(C)C)N1C(=O)C(=O)c2cc(I)cnc21</smiles>

9 ei

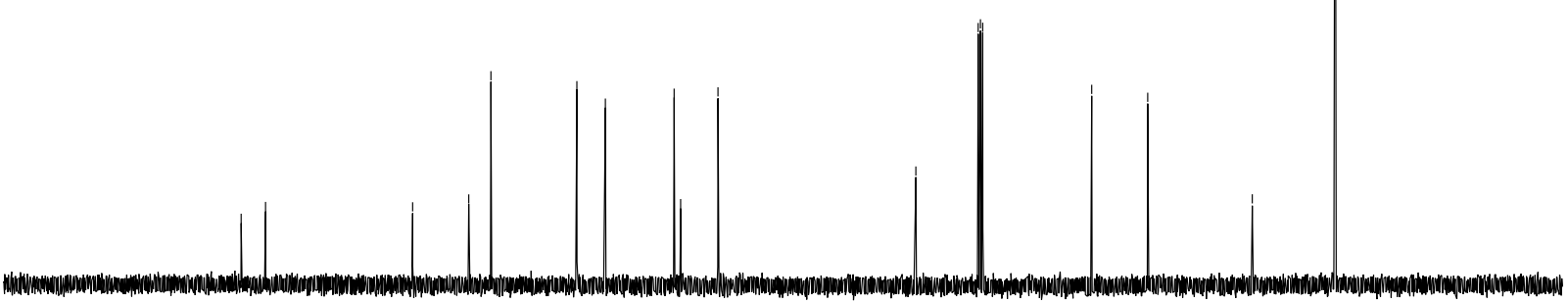

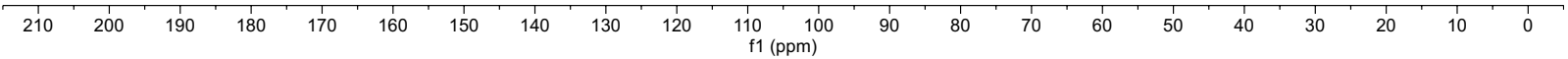


<smiles>C=CN(Nn1c(=O)c2nccnc2n1Cc1ccccc1)N1C(=O)C(=O)c2cc(Cl)ccc21</smiles>

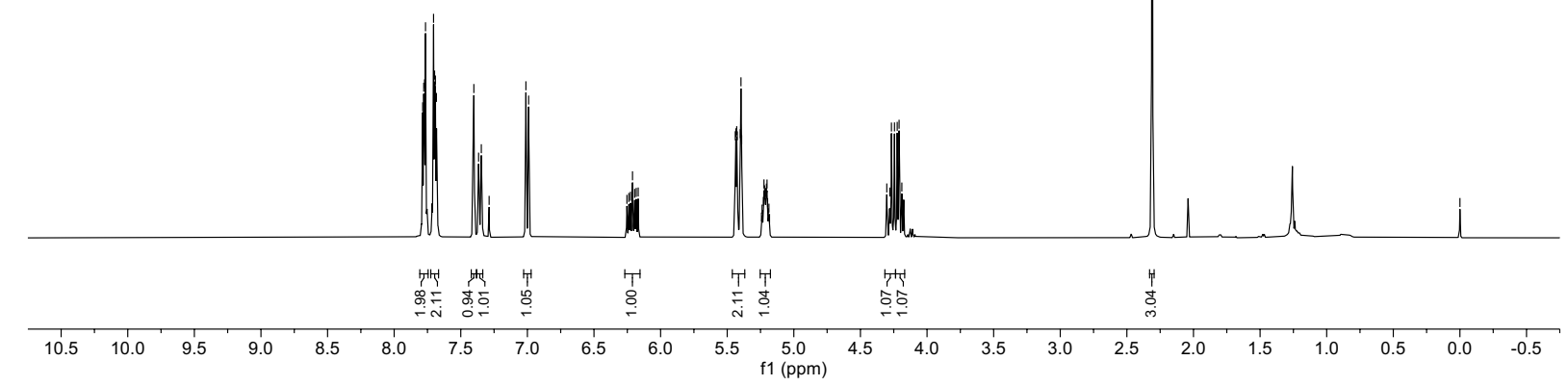

$13 \mathrm{C}(\mathrm{cdcl} 3,100.52 \mathrm{MHz})$

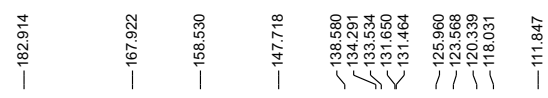

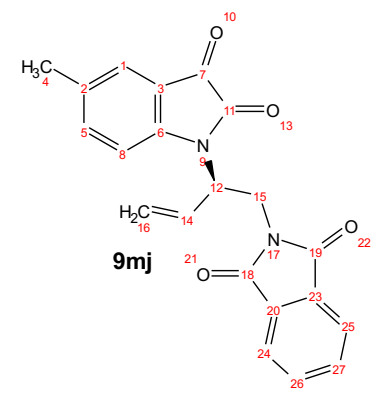

$\begin{array}{lllllllllll}210 & 200 & 190 & 180 & 170 & 160 & 150 & 140 & 130 & 120 & \begin{array}{c}\mid \\ 110 \\ \mathrm{f} 1(\mathrm{ppm})\end{array}\end{array}$ 
<smiles></smiles>

12

Y V

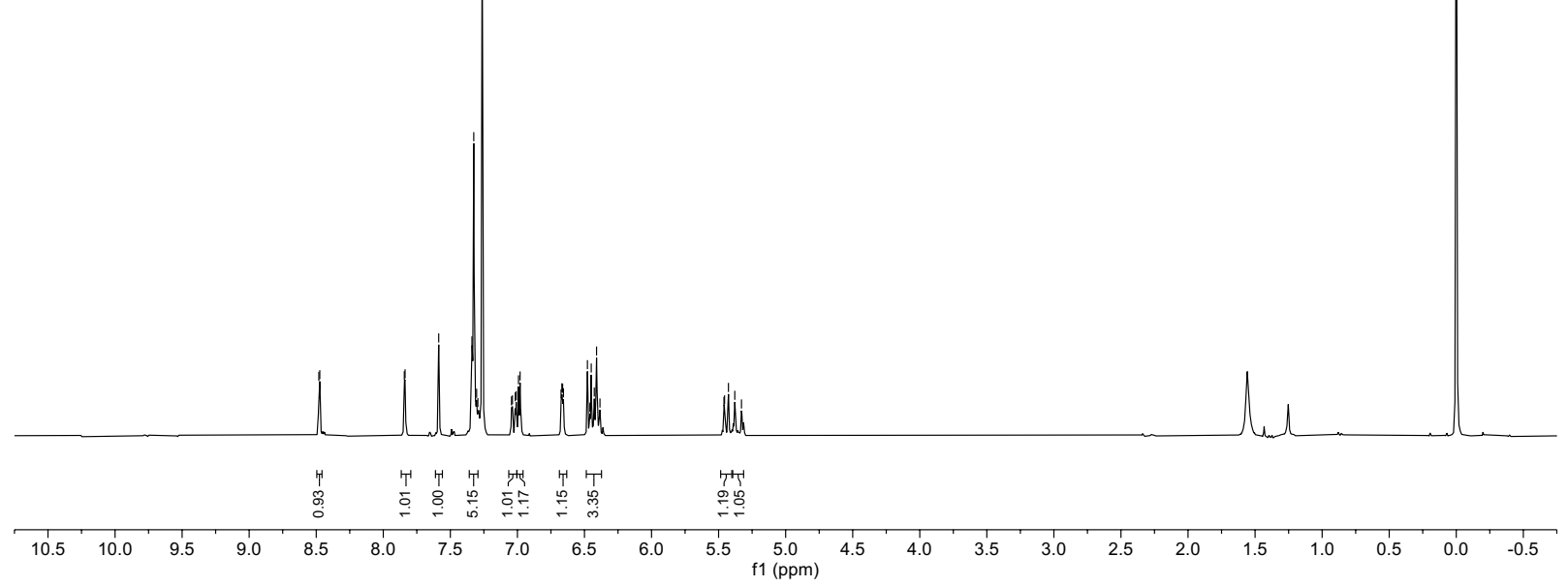

$13 \mathrm{C}(\mathrm{cdcl} 3,100.52 \mathrm{MHz})$

\begin{tabular}{|c|c|}
\hline & 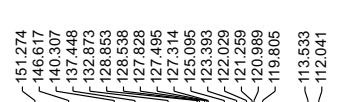 \\
\hline
\end{tabular}

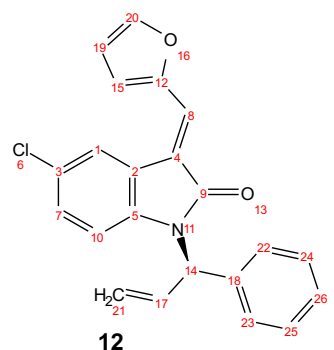

12

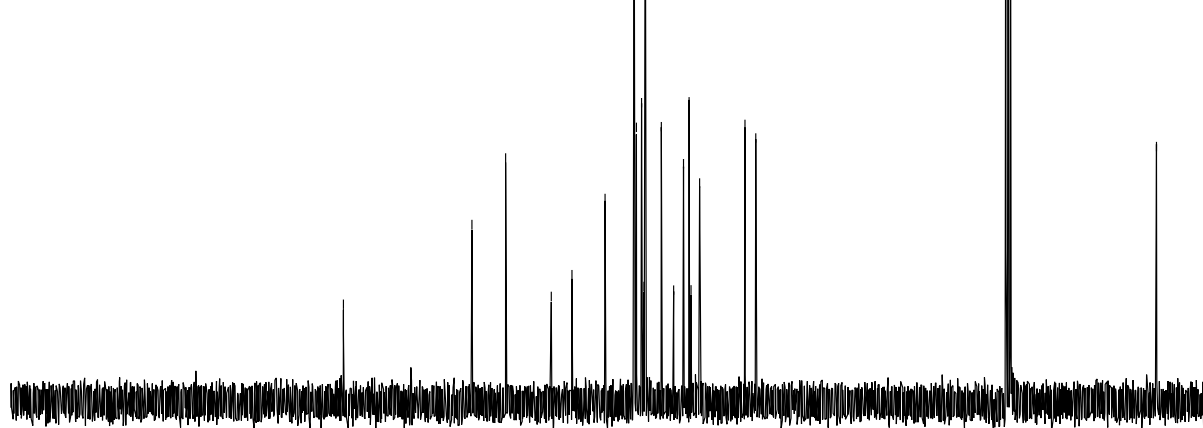

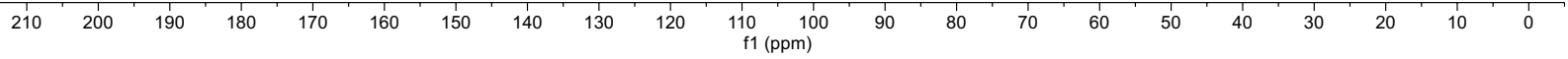


1H (CDCl3, 400.11 MHz)
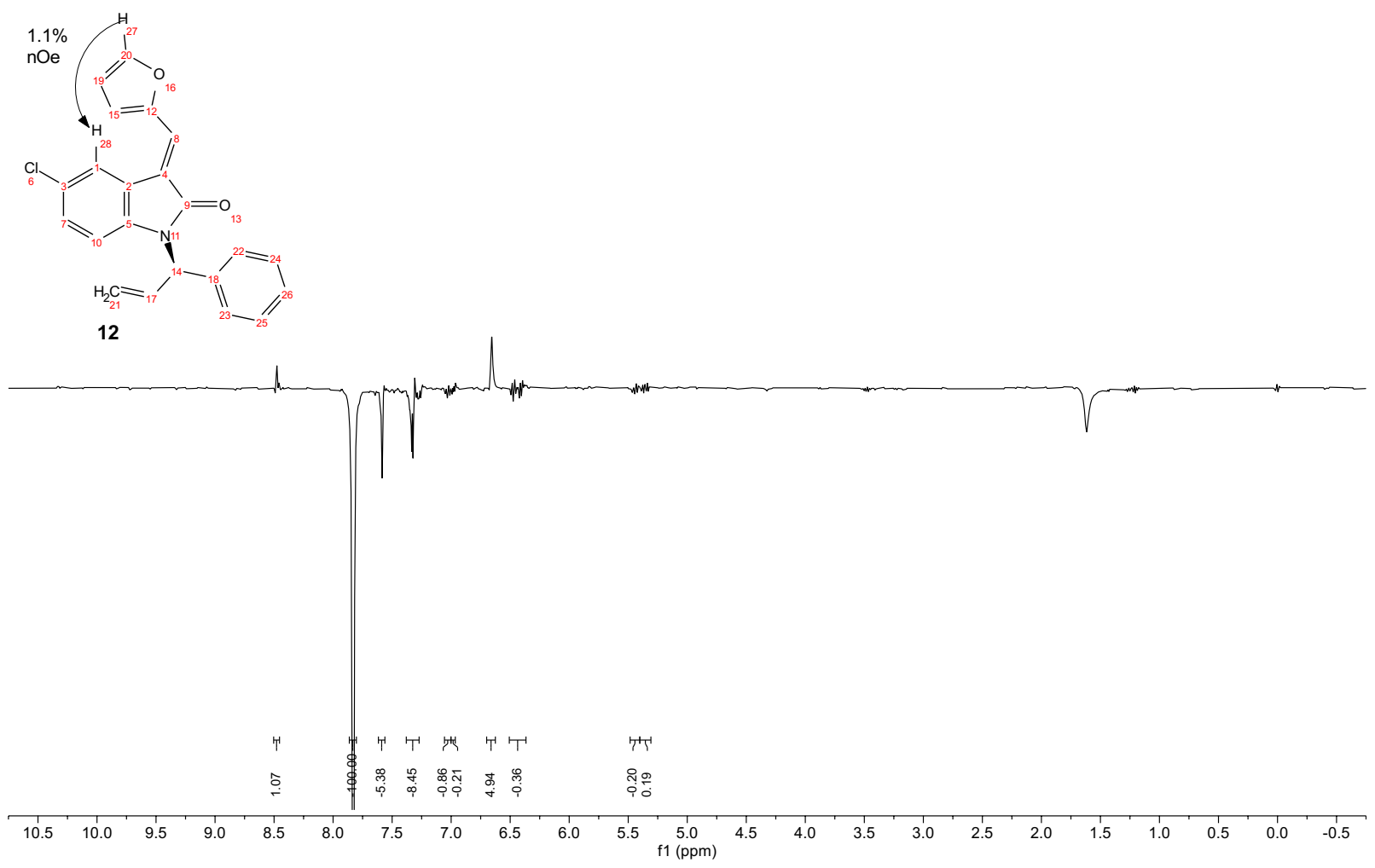

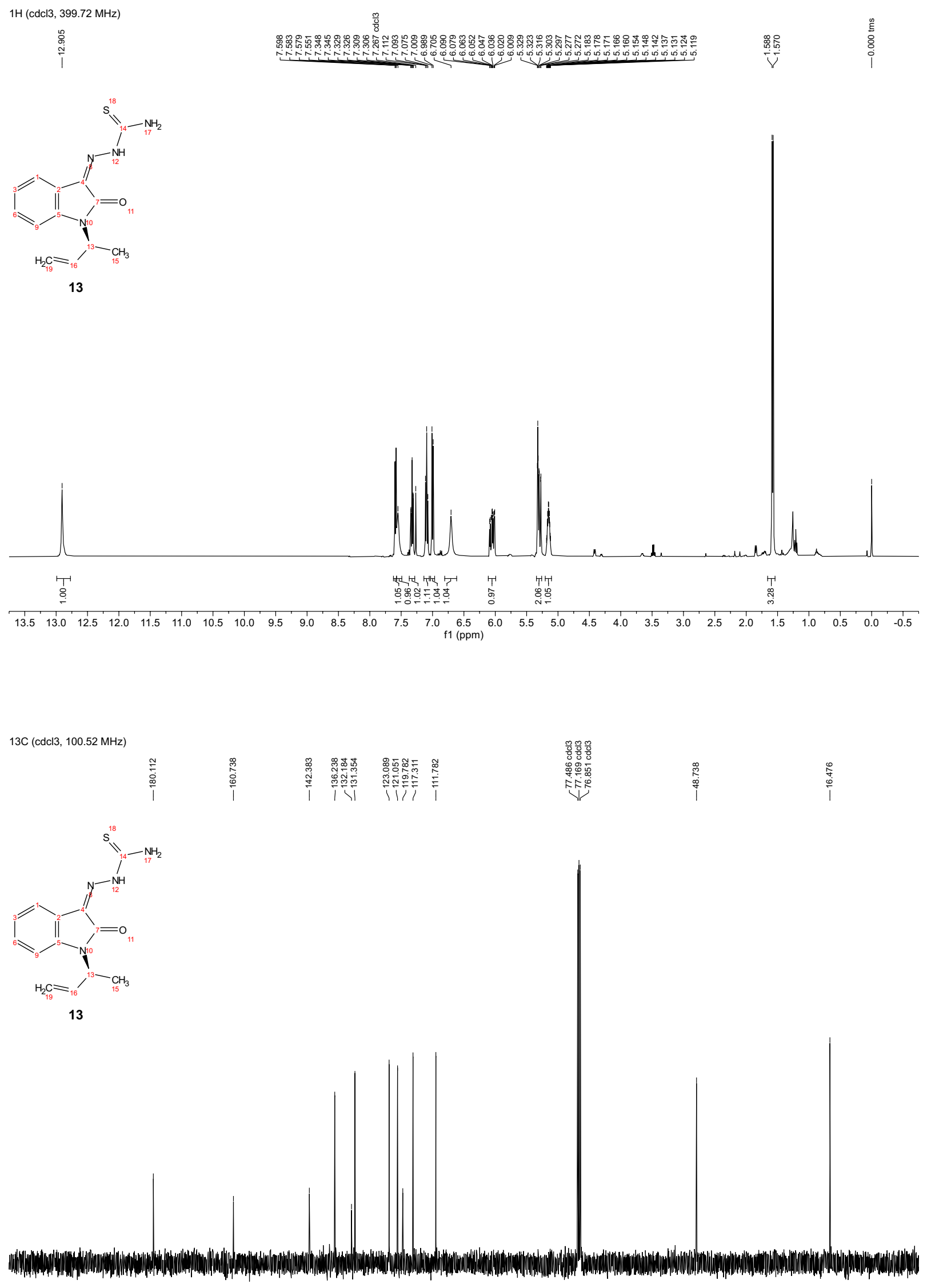

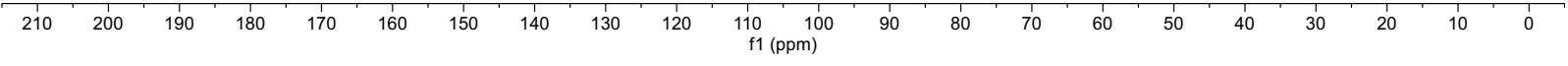


<smiles></smiles>

14

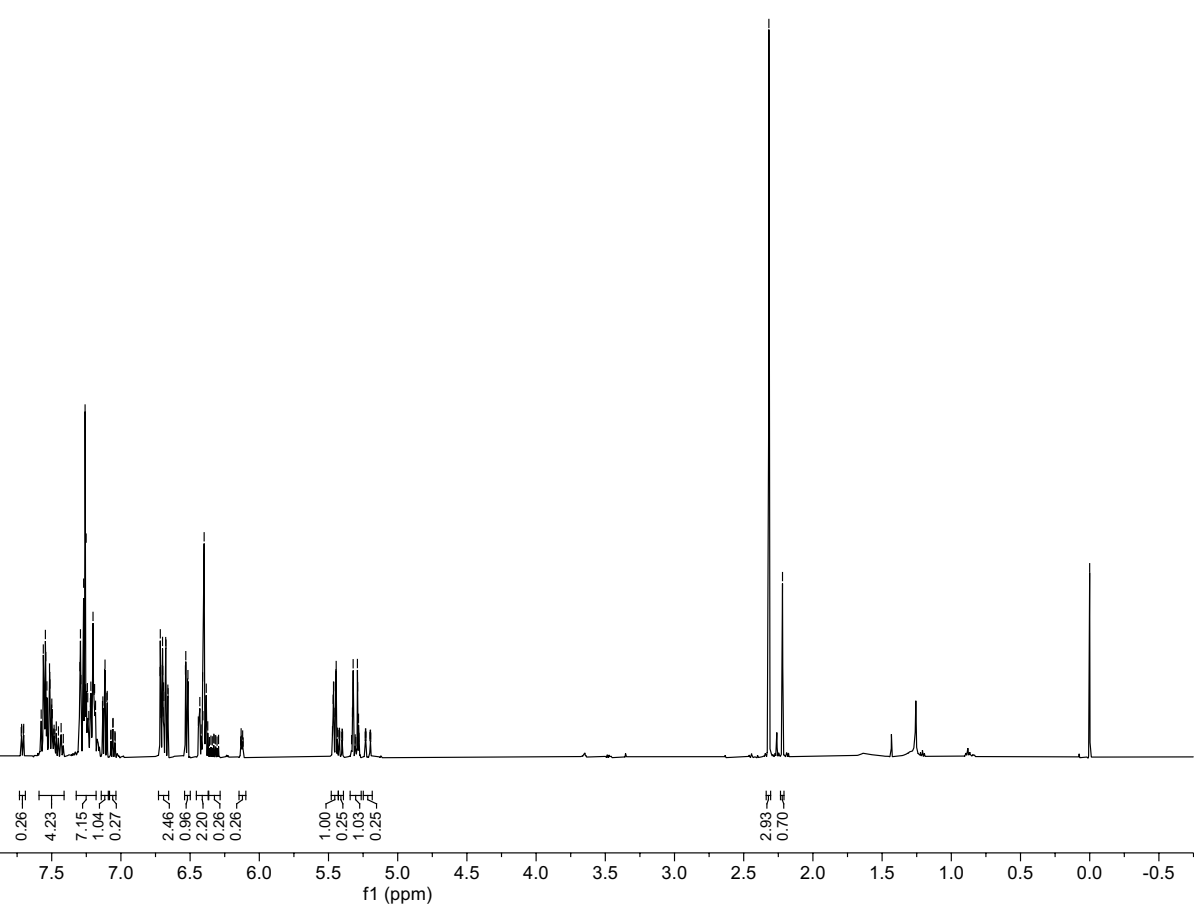

$13 \mathrm{C}(\mathrm{CDCl} 3,125.67 \mathrm{MHz})$

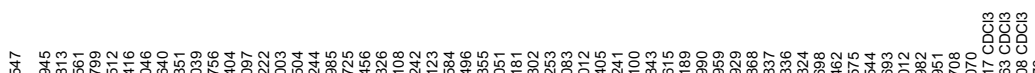

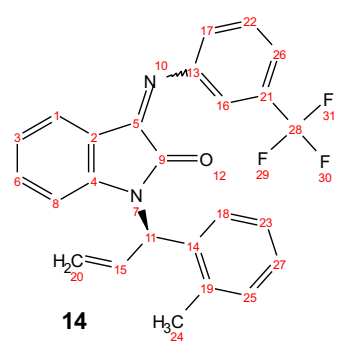

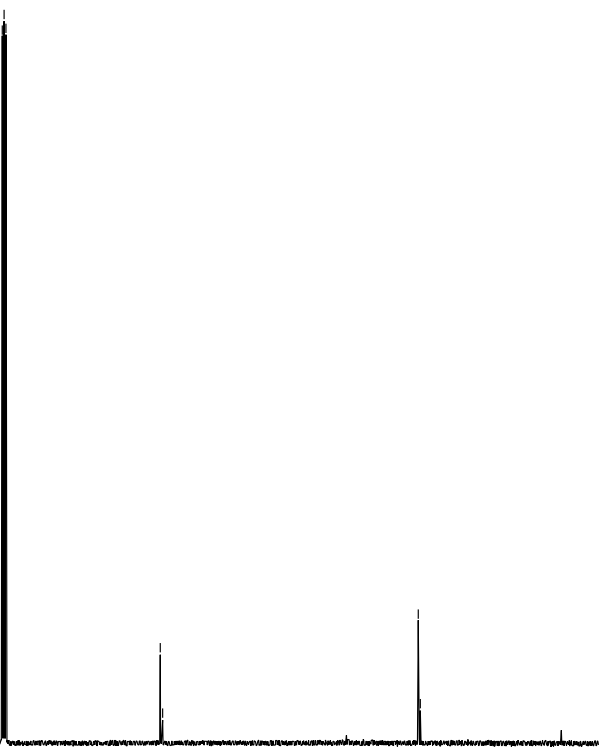

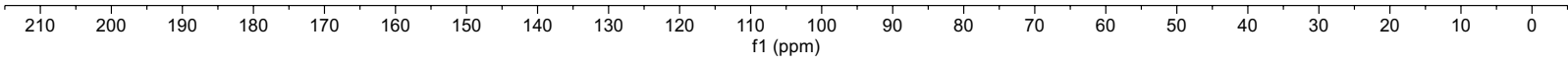




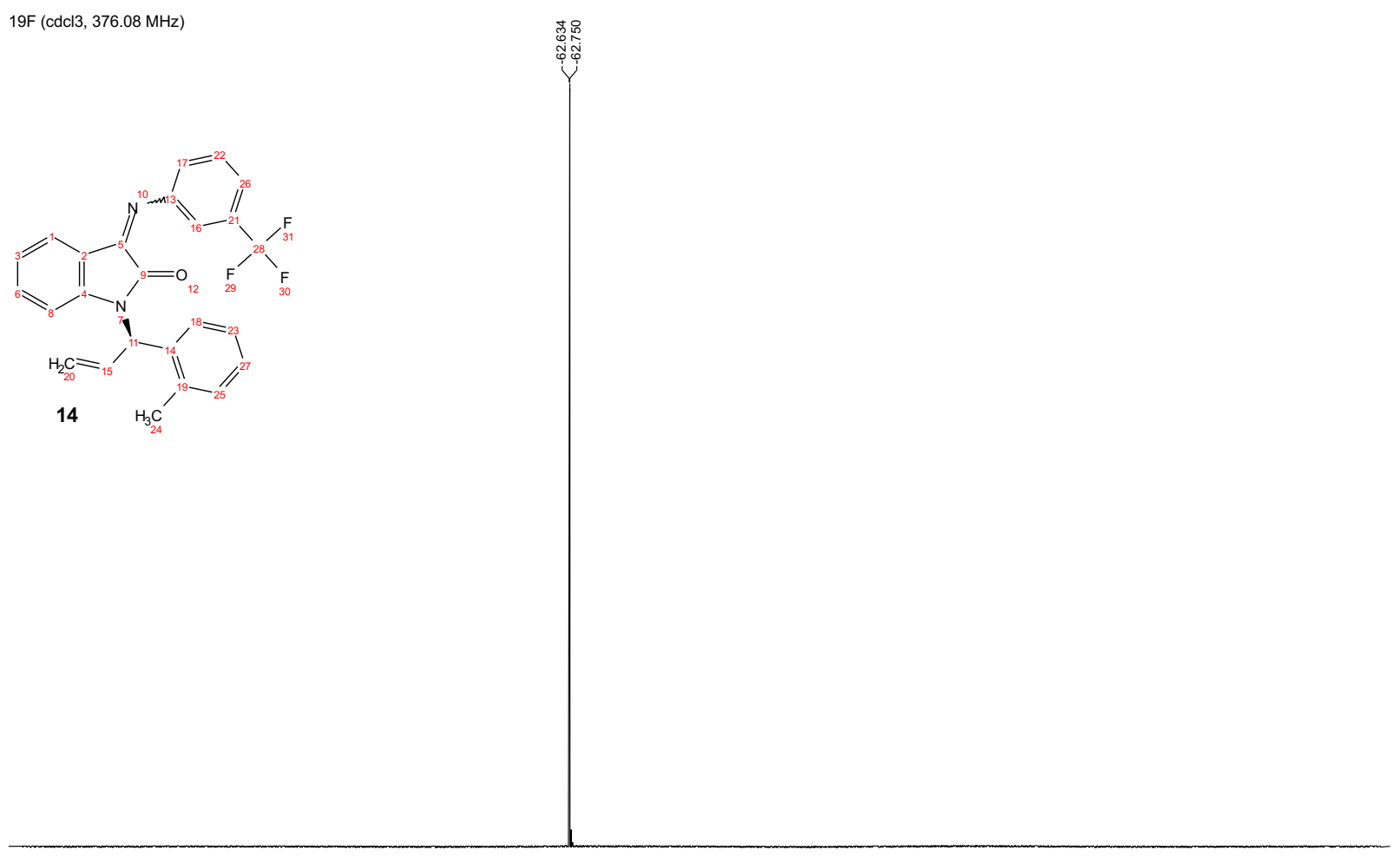

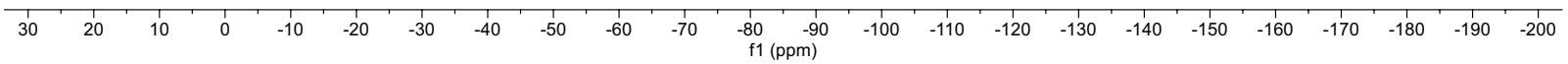



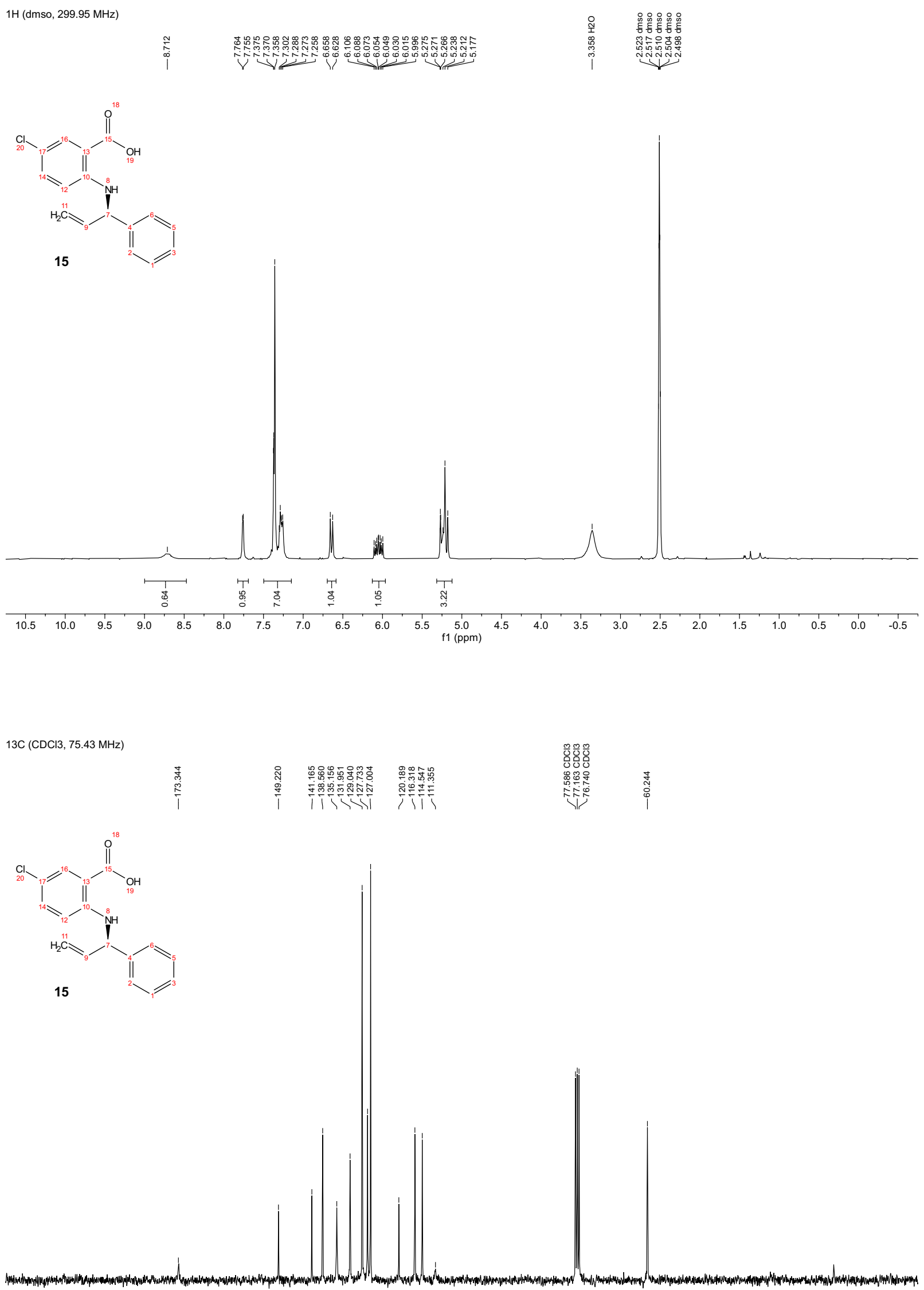

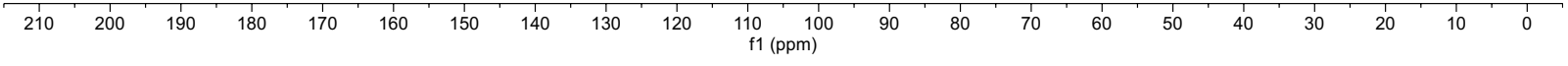


<smiles>C=CN(c1cccnc1)n1nc2c3nc4cc(O)c(Cl)cc4nc3c3ccc(Cl)cc3nc1-2</smiles>

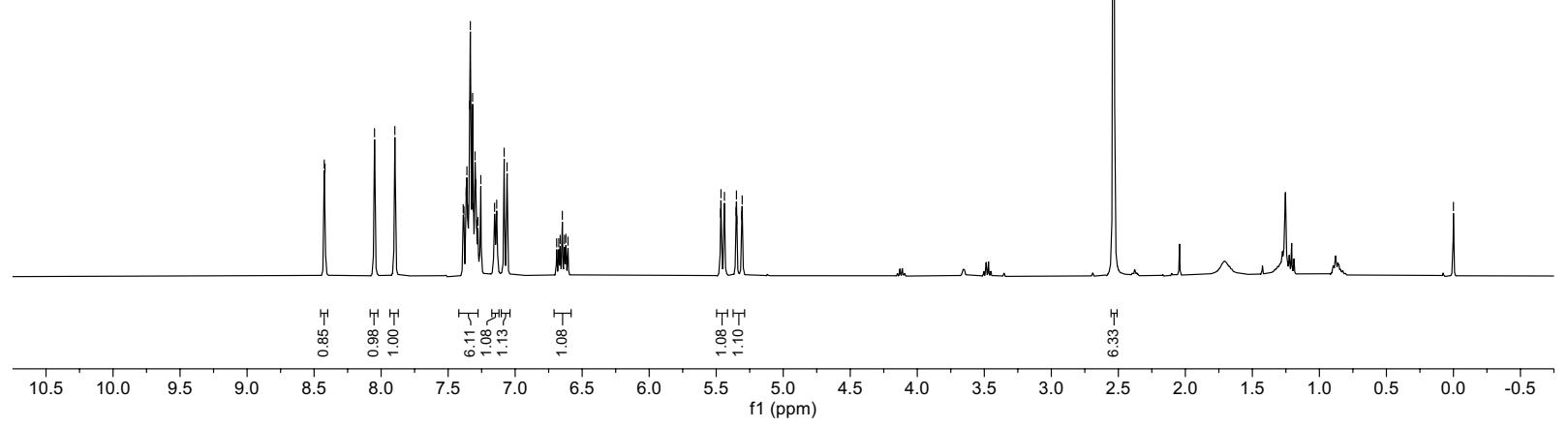

13C (cdcl3, $100.52 \mathrm{MHz}$ )
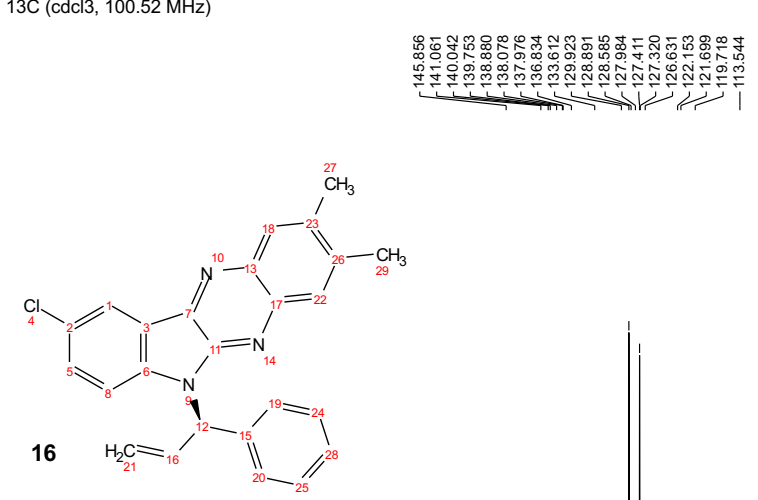

(N)
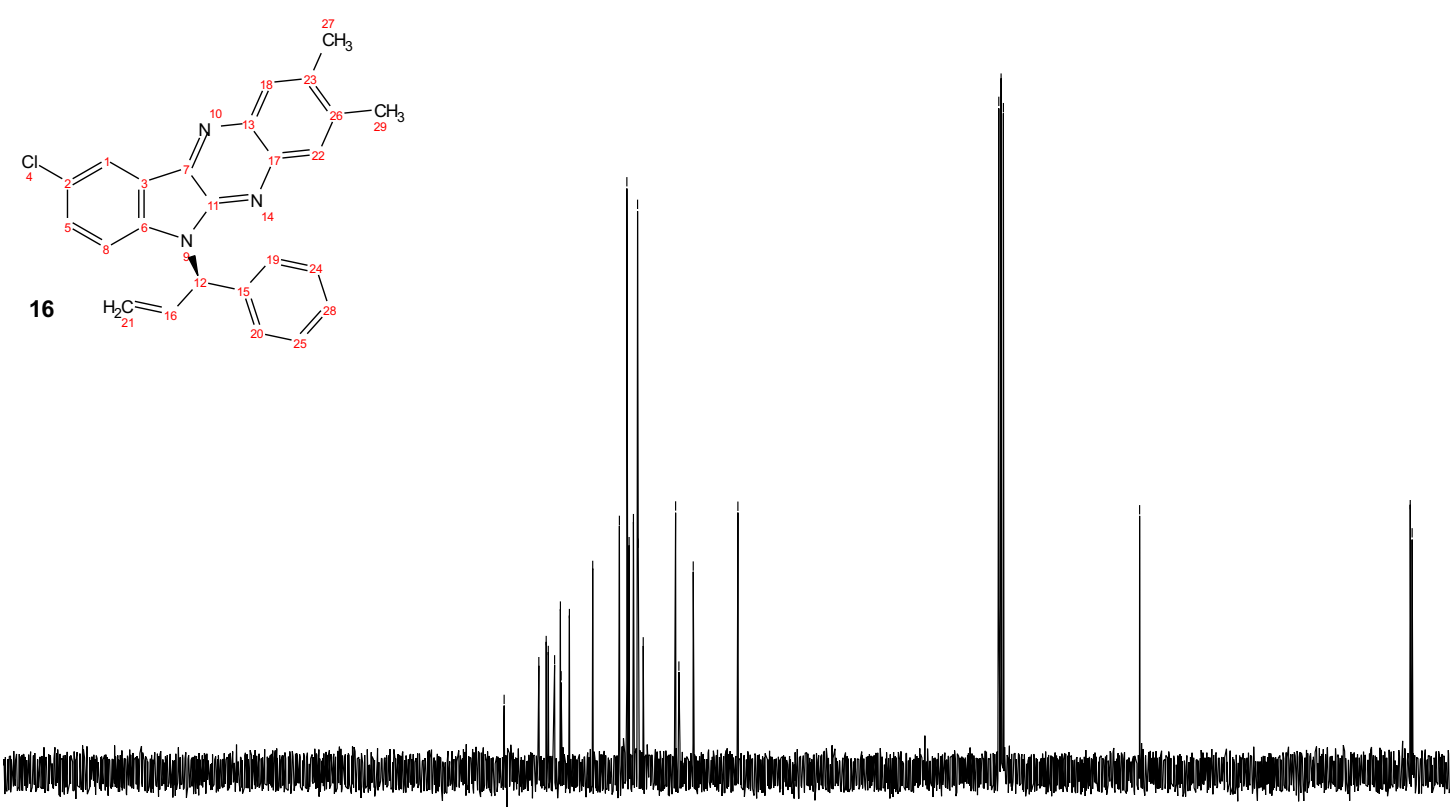

\begin{tabular}{llllllllllllllllllllllll}
\hline 10 & 200 & 190 & 180 & 170 & 160 & 150 & 140 & 130 & 120 & $\begin{array}{c}110 \\
\mathrm{f} 1(\mathrm{ppm})\end{array}$ & 90 & 80 & 70 & 60 & 50 & 40 & 30 & 20 & 10 & 0
\end{tabular} 


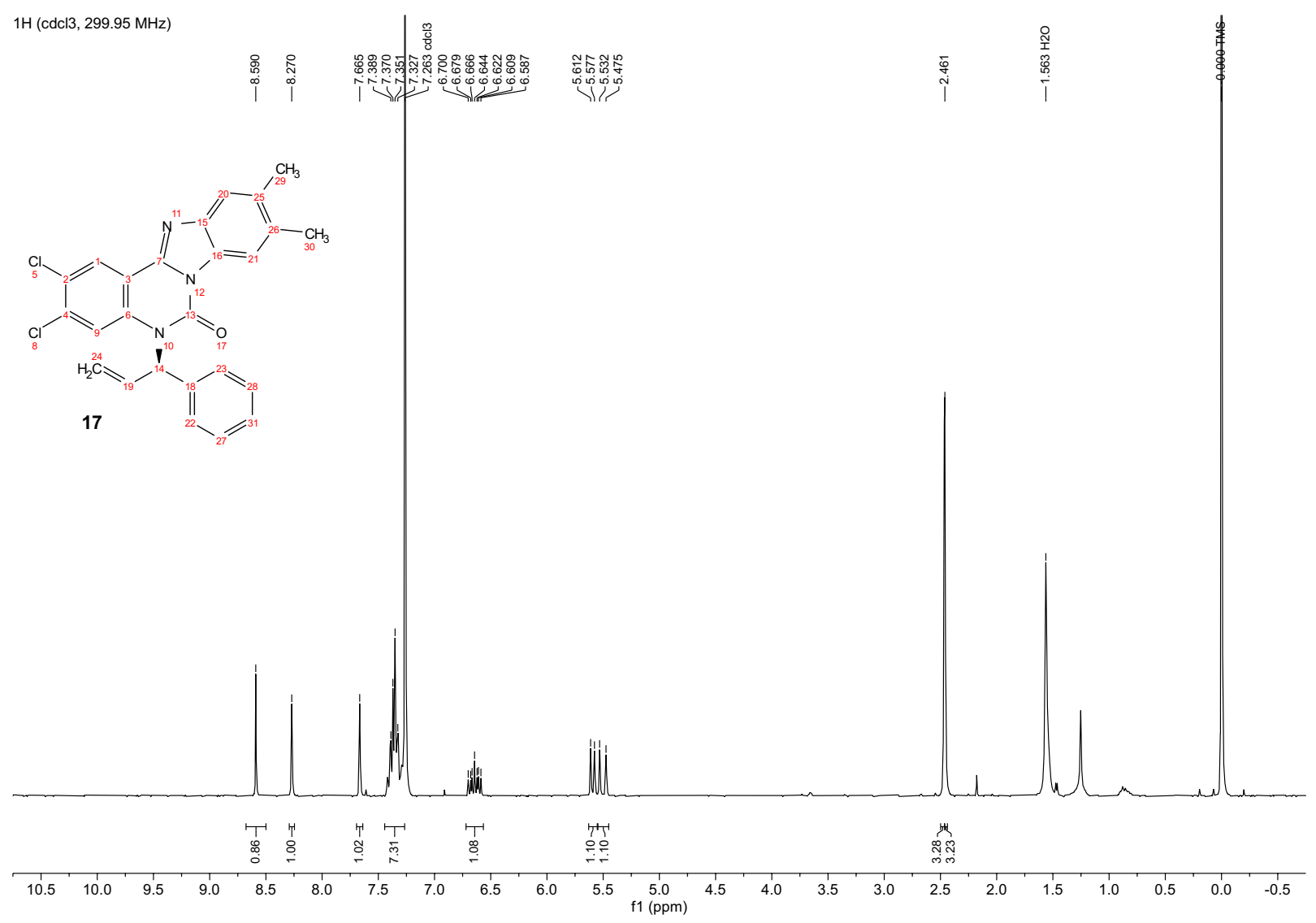

$13 \mathrm{C}(\mathrm{cdcl} 3,100.52 \mathrm{MHz})$
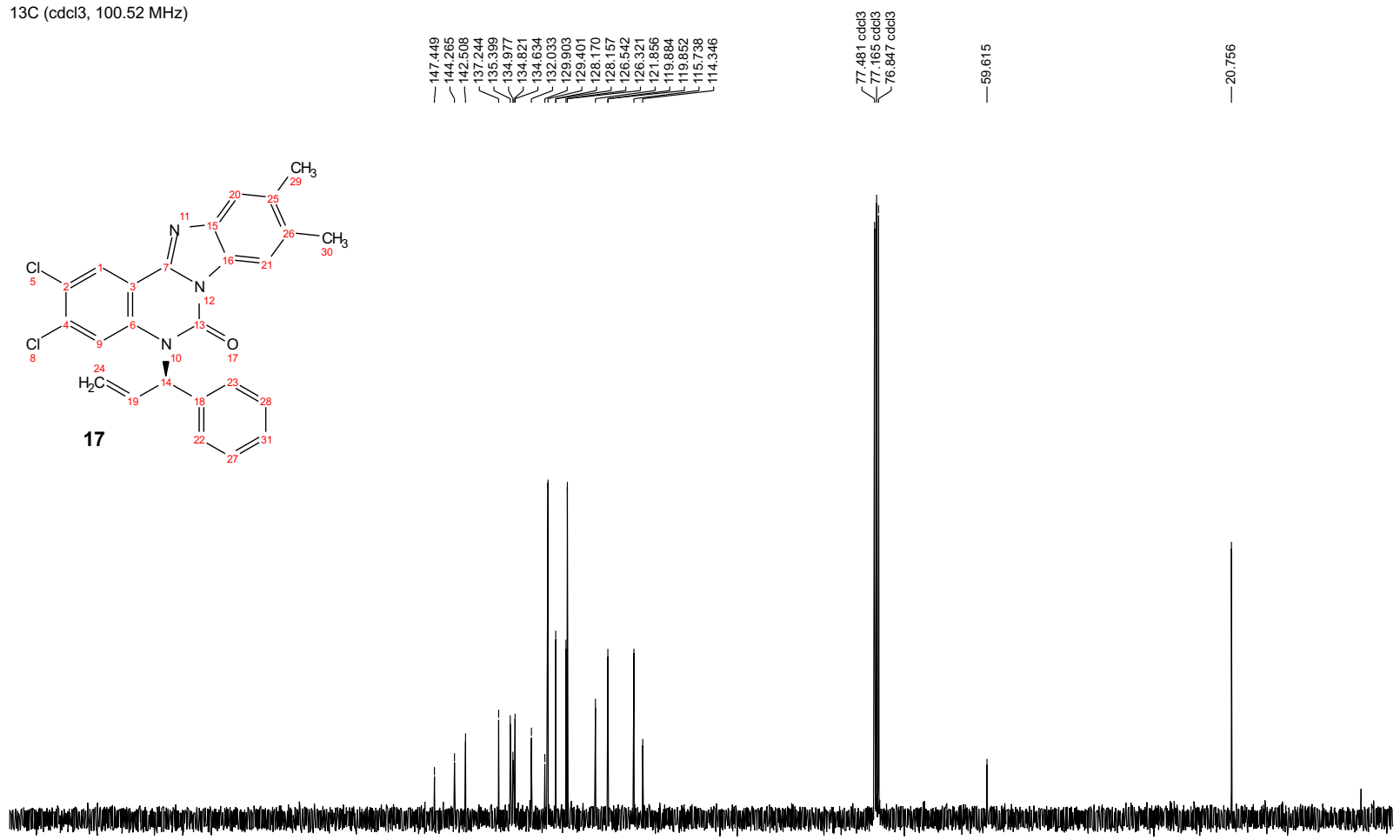

\begin{tabular}{lllllllllllllllllllll}
\hline 210 & 200 & 190 & 180 & 170 & 160 & 150 & 140 & 130 & 120 & $\begin{array}{c}110 \\
\mathrm{f} 1(\mathrm{ppm})\end{array}$ & 90 & 80 & 70 & 60 & 50 & 40 & 30 & 20 & 10 & 0
\end{tabular} 
<smiles></smiles>

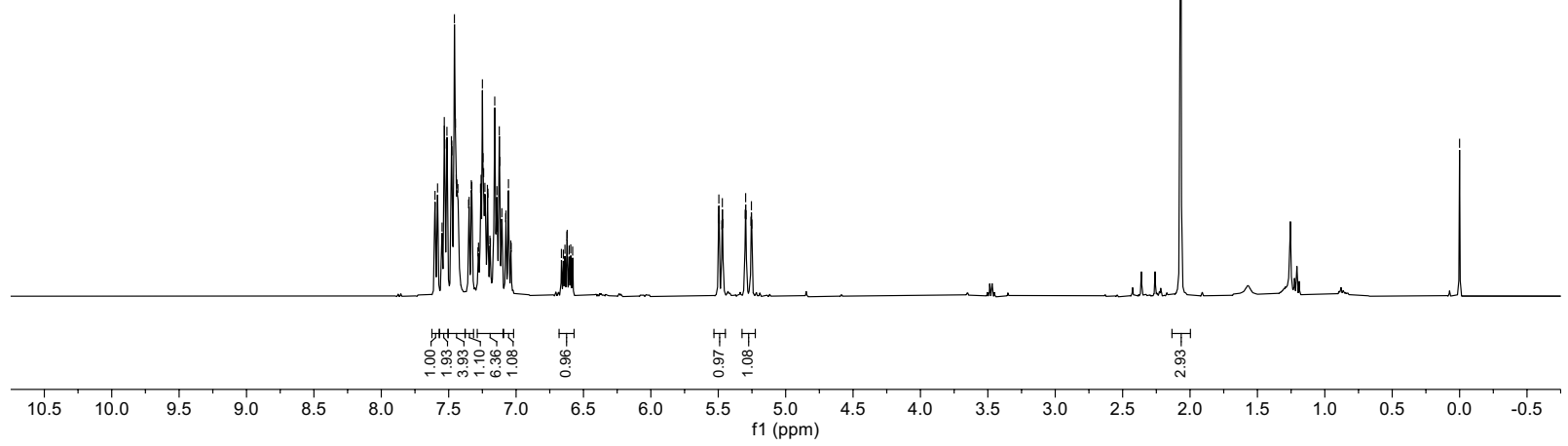

13C (cdcl3, $100.52 \mathrm{MHz})$
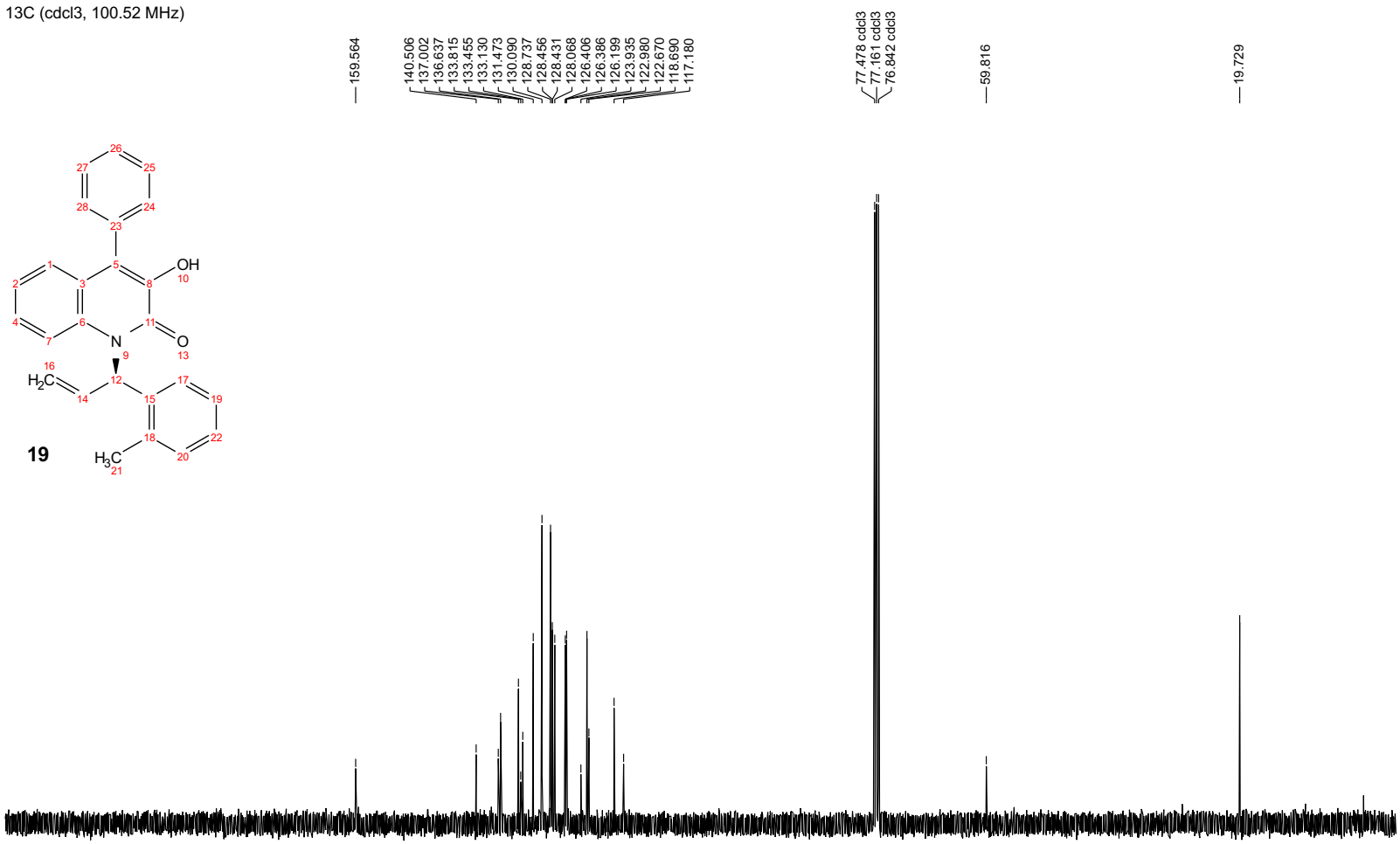

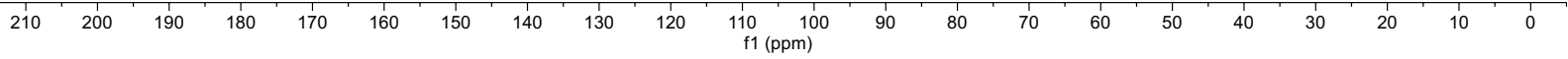



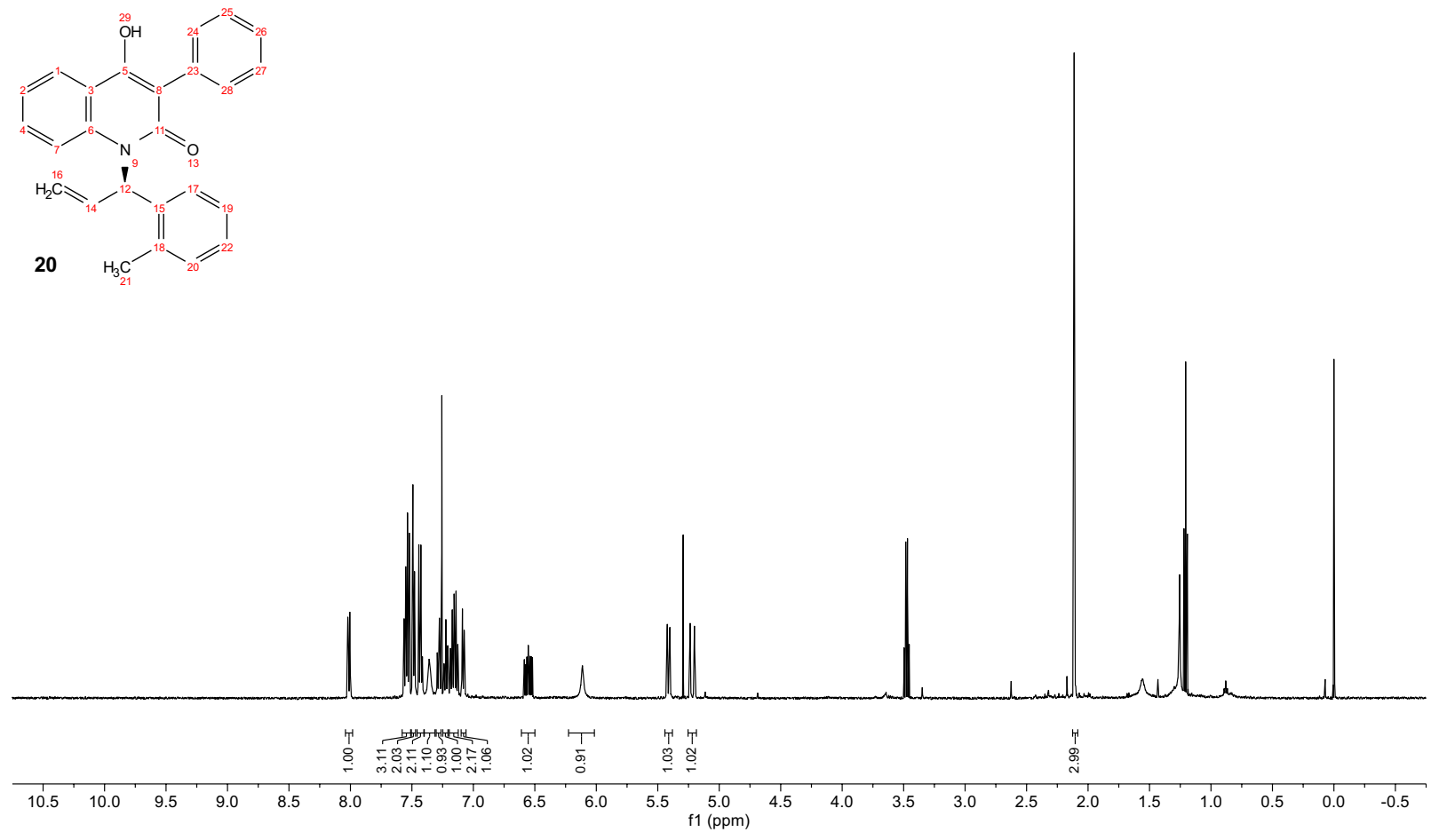

$13 \mathrm{C}(\mathrm{CDCl} 3,75.43 \mathrm{MHz})$
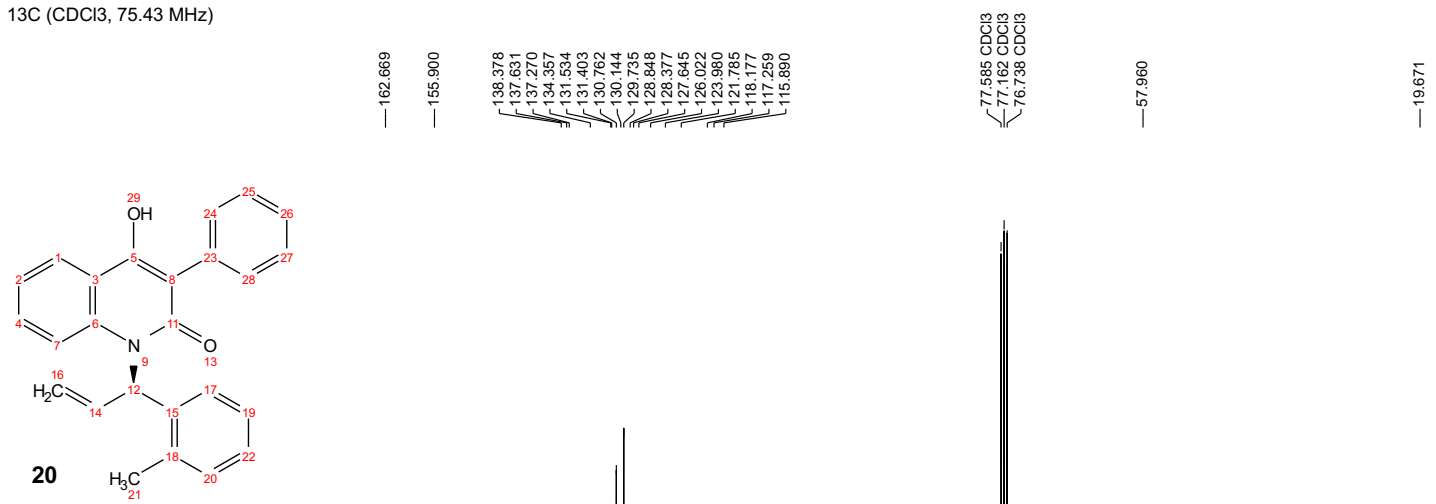

$\begin{array}{lllllllllll}210 & 200 & 190 & 180 & 170 & 160 & 150 & 140 & 130 & 120 & \begin{array}{c}110 \\ \mathrm{f} 1\end{array}(\mathrm{ppm})\end{array}$

S100 
$1 \mathrm{H}(\mathrm{CDCl} 3,499.75 \mathrm{MHz})$

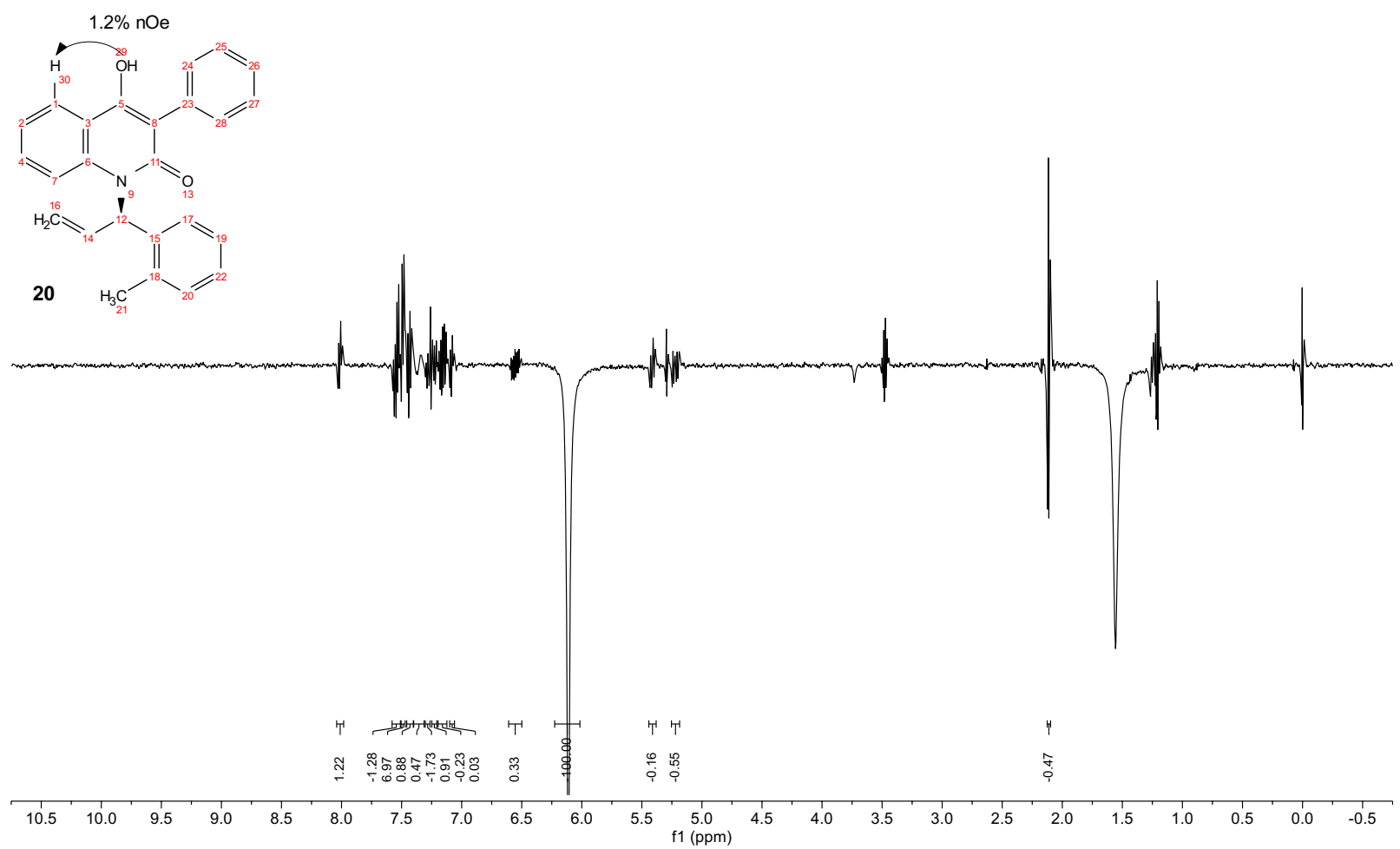

